The Intergovernmental Panel on Climate Change (IPCC) was set up jointly by the World Meteorological Organization and the 1 United Nations Environment Programme to provide an authoritative international statement of scientific understanding of climate change. The IPCC's periodic assessments of the causes, impacts and possible response strategies to climate change are the most comprehensive and up-to-date reports available on the subject, and form the standard reference for all concerned with climate change in academia, government and industry worldwide. This Synthesis Report is the fourth element of the IPCC Fourth Assessment Report "Climate Change 2007", Through three working groups, many hundreds of international experts ascess climate change in this Report. The three working group contributions are available from Cambridge University Press:

Chate Change 2007 - The Physical Science Basis

Contribution of Working Group I to the Fourth Assessment Report of the IPCC

(ISBN 9780521 88009-1 Hardback; 9780521 70596-7 Paperback)

Climate Change 2007 - Impacts, Adaptation and Vulnerability

Contribution of Working Group II to the Fourth Assessment Report of the IPCC

(978 0521 88010-7 Hardback; 9780521 70597-4 Paperback)

of Climate Change

Contribution of Working Group III to the Fourth Assessment Report of the IPCC

(978 0521 88011-4 Hardback; 9780521 70598-1

Climate Change 2007 - Synthesis Report is based on the assessment carried out by the three Working Groups of the IPCC. It provides an integrated view of climate change and addresses the following topics:

- Observed changes in climate and their effects

- Causes of change

- Climate change and its impacts in the near and long term under different scenarios

- Adaptation and mitigation options and responses, and the interrelationship with sustainable development, at global and regional levels

The long-term perspective: scientific and socio-economic aspects relevant to adaptation and mitigation, consistent with the objectives and provisions of the Convention, and in the context of sustainable development

Robust findings, key uncertaintic

\section{CLIMATE CHANGE 2007}

\section{SYNTHESIS REPORT}

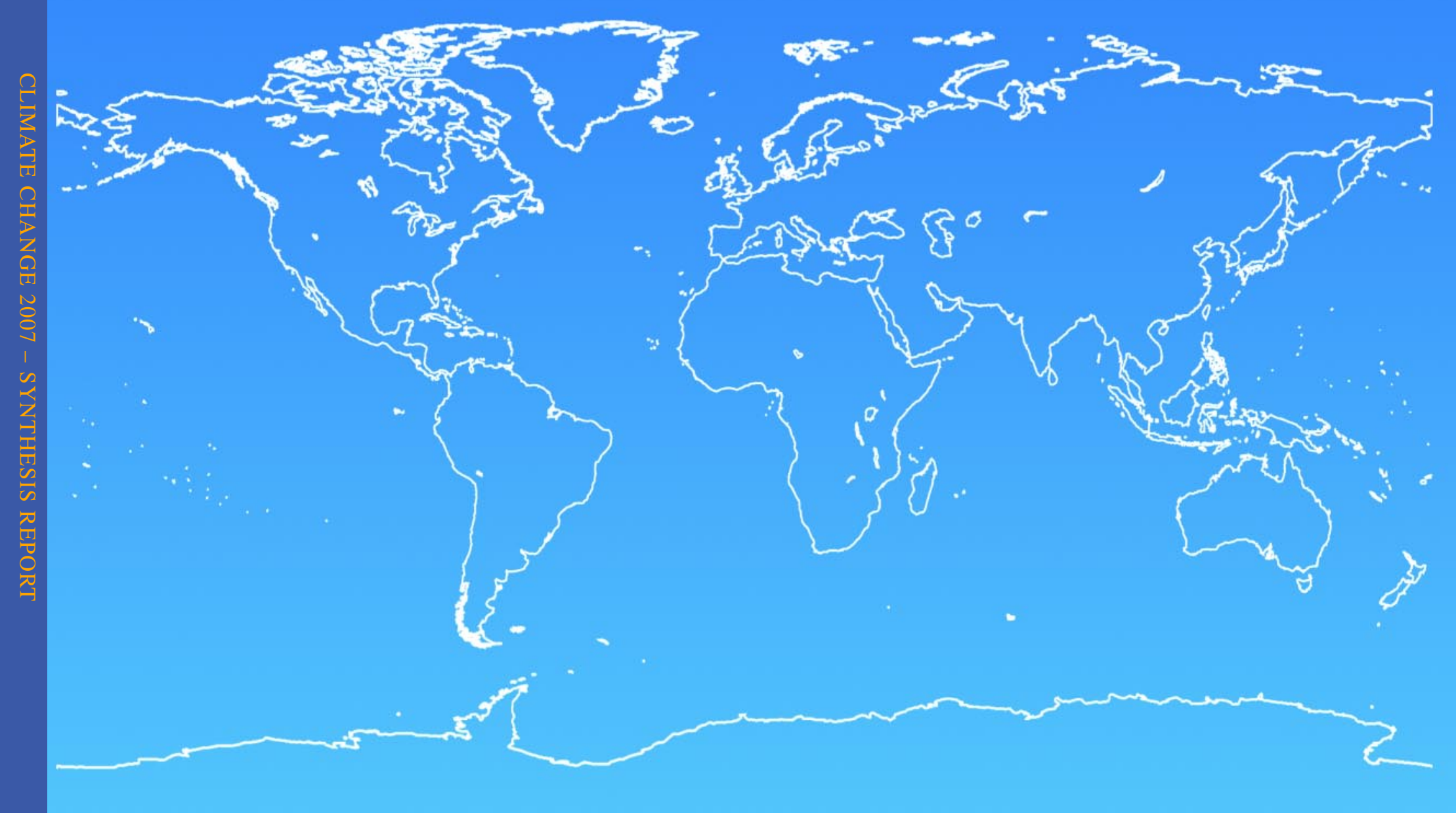

A Report of the Intergovernmental Panel on Climate Change 


\section{Climate Change 2007 Synthesis Report}

Edited by

The Core Writing Team

Synthesis Report

IPCC

\author{
Rajendra K. Pachauri \\ Chairman \\ IPCC
}

Andy Reisinger

Head, Technical Support Unit

Synthesis Report, IPCC

\section{Core Writing Team}

Lenny Bernstein, Peter Bosch, Osvaldo Canziani, Zhenlin Chen, Renate Christ, Ogunlade Davidson, William Hare, Saleemul Huq, David Karoly, Vladimir Kattsov, Zbigniew Kundzewicz, Jian Liu, Ulrike Lohmann, Martin Manning, Taroh Matsuno, Bettina Menne, Bert Metz, Monirul Mirza, Neville Nicholls, Leonard Nurse, Rajendra Pachauri, Jean Palutikof, Martin Parry, Dahe Qin, Nijavalli Ravindranath, Andy Reisinger, Jiawen Ren, Keywan Riahi, Cynthia Rosenzweig, Matilde Rusticucci, Stephen Schneider, Youba Sokona, Susan Solomon, Peter Stott, Ronald Stouffer, Taishi Sugiyama, Rob Swart, Dennis Tirpak, Coleen Vogel, Gary Yohe

Technical Support Unit for the Synthesis Report:

Andy Reisinger, Richard Nottage, Prima Madan

\section{Referencing this report}

IPCC, 2007: Climate Change 2007: Synthesis Report. Contribution of Working Groups I, II and III to the Fourth Assessment Report of the Intergovernmental Panel on Climate Change [Core Writing Team, Pachauri, R.K and Reisinger, A. (eds.)]. IPCC, Geneva, Switzerland, 104 pp. 


\section{PUBLISHED BY THE INTERGOVERNMENTAL PANEL ON CLIMATE CHANGE}

() Intergovernmental Panel on Climate Change, 2008

First published 2008

ISBN 92-9169-122-4

The right of publication in print, electronic and any other form and in any language is reserved by the IPCC. Short extracts from this publication may be reproduced without authorization provided that complete source is clearly indicated. Editorial correspondence and requests to publish, reproduce or translate articles in part or in whole should be addressed to:

IPCC

c/o World Meteorological Organization (WMO)

7 bis avenue de la Paix

Tel. : +4122730 8208

P.O Box No. 2300

Fax.: +41227308025

CH-1211 Geneva 2, Switzerland E-mail: IPCC-Sec@wmo.int

The presentation of material and designations employed in this publication do not imply the expression of any opinion whatsoever on the part of the IPCC concerning the legal status of any country, territory, city or area or of its authorities, or concerning the delimitation of its frontiers or boundaries.

The mention of specific companies or products does not imply that they are endorsed or recommended by IPCC in preference to others of a similar nature which are not mentioned or advertised.

Typeface Times New Roman 9.5/11.5. System Adobe PageMaker 6.5

Layout and Graphics Design Support: TERI Press

Printed in Sweden

The Intergovernmental Panel on Climate Change was joint recipient of the 2007 Nobel Peace Prize

(C) The Nobel Foundation. Nobel Prize ${ }^{\circledR}$ and the Nobel Prize ${ }^{\circledR}$ medal design are registered trademarks of the Nobel Foundation 


\section{Foreword}

The Intergovernmental Panel on Climate Change (IPCC) was jointly established in 1988, by the World Meteorological Organization (WMO) and the United Nations Environment Programme (UNEP), with the mandate to assess scientific information related to climate change, to evaluate the environmental and socio-economic consequences of climate change, and to formulate realistic response strategies. The IPCC multivolume assessments have since then played a major role in assisting governments to adopt and implement policies in response to climate change, and in particular have responded to the need for authoritative advice of the Conference of the Parties (COP) to the United Nations Framework Convention on Climate Change (UNFCCC), which was established in 1992, and its 1997 Kyoto Protocol.

Since its establishment, the IPCC has produced a series of Assessment Reports (1990, 1995, 2001 and this one in 2007), Special Reports, Technical Papers and Methodology Reports, which have become standard works of reference, widely used by policymakers, scientists, other experts and students. The most recent publications include a Special Report on "Carbon Dioxide Capture and Storage" and one on "Safeguarding the Ozone Layer and the Global Climate System", published in 2005, and the "Guidelines for National Greenhouse Gas Inventories" re-edited in 2006. A Technical Paper on "Climate Change and Water" is under preparation.

This Synthesis Report (SYR), adopted in Valencia, Spain, on 17 November 2007, completes the four-volume Fourth Assessment Report (AR4), which was released in various steps throughout the year under the title "Climate Change 2007". It summarises the findings of the three Working Group reports and provides a synthesis that specifically addresses the issues of concern to policymakers in the domain of climate change: it confirms that climate change is occurring now, mostly as a result of human activities; it illustrates the impacts of global warming already under way and to be expected in future, and describes the potential for adaptation of society to reduce its vulnerability; finally it presents an analysis of costs, policies and technologies intended to limit the extent of future changes in the climate system.

The AR4 is a remarkable achievement involving more than 500 Lead Authors and 2000 Expert Reviewers, building on the work of a wide scientific community and submitted to the scrutiny of delegates from more than one hundred participating nations. It is the result of the enthusiasm, dedication, and cooperation of experts from many different but related disciplines. We would like to express our gratitude to all of them, to the Members of the IPCC Bureau, to the staff of the Technical Support Units, particularly of the Technical Support Unit for the IPCC Synthesis Report in The Energy and Resources Institute (TERI) in Delhi, to Dr Renate Christ, Secretary of the IPCC, and to the Secretariat staff.

We acknowledge with gratitude the governments and organisations that contribute to the IPCC Trust Fund and provide support to experts in different ways. The IPCC has been especially successful in engaging in its work a large number of experts from the developing countries and countries with economies in transition; the Trust Fund enables extending financial assistance for their travel to IPCC meetings. We also acknowledge the cooperative spirit in which all government delegates have worked together in the IPCC Sessions to reach a meaningful and powerful consensus.

Finally, we would like to thank the Chairman of the IPCC, Dr Rajendra K. Pachauri, for leading tirelessly and with dedication the effort of all. This is particularly appropriate at this time as the IPCC as a whole, under his guidance, has been awarded the 2007 Nobel Peace Price.

We would also like at this occasion to express deep recognition and sorrow in remembrance of Prof. Bert Bolin, who led the way twenty years ago as first Chairman of IPCC, and who sadly passed away on 30 December 2007 after a brilliant career in meteorology and climate science.

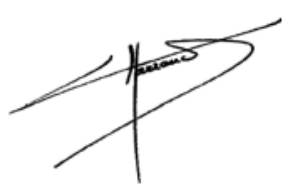

Michel Jarraud

Secretary General

World Meteorological Organization

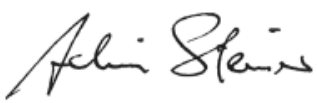

Achim Steiner

Executive Director

United Nations Environmental Programme 



\section{Preface}

This Synthesis Report with its Summary for Policymakers is the fourth and final part of the Fourth Assessment Report (AR4) of the Intergovernmental Panel on Climate Change (IPCC) - "Climate Change 2007". It draws together and integrates for the benefit of policy makers and those from others professions, up to date policy-relevant scientific, technical and socio-economic information on climate change. This report is intended to assist governments and other decision-makers in the public and private sector in formulating and implementing appropriate responses to the threat of human-induced climate change.

The scope of the Synthesis Report includes the information contained in the three Working Group contributions to the IPCC AR4, the Working Group I report on "The Physical Science Basis", the Working Group II report on "Impacts, Adaptation and Vulnerability" and the Working Group III report on "Mitigation of Climate Change". It also draws on other IPCC reports, in particular recently published IPCC Special Reports. The Synthesis Report was written by a team dedicated to this task drawn from the authors of each Working Group report of the AR4 led by the Chair of the IPCC. As instructed by the Panel, the authors prepared the draft in a non-technical style while ensuring that scientific and technical facts are recorded correctly.

The Synthesis Report addresses a range of broad policy-relevant questions, structured around 6 topic headings agreed by the Panel, and it gives careful attention to cross-cutting themes. It consists of two parts, a Summary for Policymakers (SPM) and a longer report. The sections of the SPM follow largely the topic structure of the longer report, but for brevity and clarity, certain issues covered in more than one topic are summarised in one section of the SPM.

Topic 1 brings together information from Working Groups I and II on observed changes in climate and the effects of past climate change on natural systems and human society.

Topic 2 addresses causes of change, considering both natural and anthropogenic drivers of climate change. It analyses the chain including greenhouse gas emissions and concentrations, to radiative forcing and resultant climate change, and evaluates whether observed changes in climate and in physical and biological systems can be attributed to natural or anthropogenic causes. In providing that information it draws on information contained in all three Working Group contributions to the AR4.

Topic 3 presents information from the three Working Group reports on projected future climate change and its impacts. It provides updated information on emissions scenarios and pro- jected future changes in climate in the $21^{\text {st }}$ century and beyond, and describes the projected impacts of future climate change on systems, sectors and regions. Special attention is given to issues of human well-being and development.

Topic 4 describes adaptation and mitigation options and responses as assessed in the reports of Working Groups II and III, and the inter-relationship of climate change and response measures with sustainable development. The focus of this topic is on response measures that can be implemented by 2030 . Technologies, policies, measures and instruments as well as barriers to implementation are addressed along with synergies and trade-offs.

Topic 5 covers the long-term perspective and analyses scientific, technical and socio-economic aspects relevant to adaptation and mitigation, consistent with the objectives and provisions of the United Nations Framework Convention on Climate Change (UNFCCC). It places decision-making about climate change in a risk management perspective, paying attention to broader environmental and integration issues. The topic describes emissions trajectories for stabilisation of greenhouse gas concentrations at various levels and associated temperature increases, along with information about the costs of mitigation, required technology development and deployment, and avoided climate impacts. It also explores in detail five main reasons for concern regarding climate change, which it finds have become stronger due to new knowledge since the TAR.

Topic 6 highlights robust findings and key uncertainties.

While the Synthesis Report is a largely self-contained document, it needs to be viewed in the context of the other volumes of "Climate Change 2007" and it is recommended that for further details the contributions of the three Working Groups be consulted. Each of the Working Group reports consists of a series of chapters which contain a detailed scientific technical assessment, a Technical Summary, and a Summary for Policymakers that has been approved by the IPCC line by line.

The longer Synthesis Report contains extensive references to the relevant chapters in the Working Group contributions to the AR4 and other relevant IPCC reports. For easy reading, references in the Summary for Policymakers only lead to the relevant sections of the longer Synthesis Report. The CD RoM included in this report contains the full text of the three Working Group contributions to the AR4 in English, as well as the Summaries for Policymakers and Technical Summaries and the Synthesis Report in all official UN languages. References in these electronic versions are provided as hyperlinks to en- 
able the reader to easily find further scientific, technical and socio-economic information. A user guide, glossary of terms, and lists of acronyms, authors, Review Editors and Expert Reviewers are provided in the Annexes to this report.

The preparation of the Synthesis Report was carried out in accordance with the Procedures for the Preparation, Review, Acceptance, Adoption, Approval and Publication of IPCC Reports, and it was adopted and approved by the IPCC at its Twenty Seventh Session (Valencia, Spain, 12-17 November 2007).

We take this opportunity to thank:

- the Core Writing Team who drafted this report and, with their meticulous and painstaking attention to detail, finalised it

- the Review Editors who made sure that all comments were taken into consideration and that consistency with the underlying reports was maintained

- the members of the Working Groups' teams of Coordinating Lead Authors and Lead Authors who helped with the drafting

- the Head and staff of the SYR Technical Support Unit, particularly Dr Andy Reisinger, and the Technical Support Units of the three Working Groups, for logistical and editorial support

- the staff of the IPCC Secretariat for innumerable tasks performed in support of the preparation, release and publication of the report
- WMO and UNEP for supporting the IPCC Secretariat and for financial contributions to the IPCC Trust Fund

- all member governments and the UNFCCC for their contributions to the IPCC Trust Fund

- and all member governments and participating organisations for invaluable in-kind contributions, including through supporting experts involved in the IPCC process and hosting meetings and sessions of the IPCC.

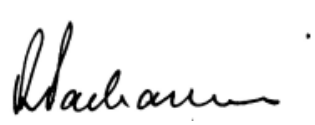

Dr. R.K Pachauri

Chairman of the IPCC

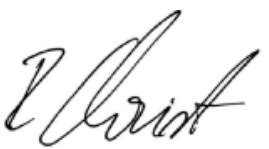

Dr. Renate Christ

Secretary of the IPCC 


\section{Climate Change 2007: Synthesis Report}

\section{Contents}

Foreword

Preface

Summary for Policymakers

Synthesis Report $\quad 23$

Introduction $\quad 25$

Topic 1

29

Topic 2

Topic 3

Topic 4

Topic 5

Topic 6

\section{Annexes}

I. User guide and access to more detailed information $\quad 75$

II. Glossary $\quad 76$

III. Acronyms, chemical symbols; scientific units; country groupings 90

IV. List of authors $\quad 92$

V. List of Reviewers and Review Editors $\quad 94$

$\begin{array}{lr}\text { VI. Index } & 100\end{array}$

VII. List of Publications by the Intergovernmental Panel on Climate Change 102 


\section{Sources cited in this Synthesis Report}

References for material contained in this report are given in curly brackets \{\} at the end of each paragraph.

In the Summary for Policymakers, references refer to Sections, Figures, Tables and Boxes in the underlying Introduction and Topics of this Synthesis Report.

In the Introduction and six Topics of this Synthesis Report, references refer to the contributions of Working Groups I, II and III (WGI, WGII and WGIII) to the Fourth Assessment Report and other IPCC Reports on which this Synthesis Report is based, or to other Sections of the Synthesis Report itself (SYR).

The following abbreviations have been used:

SPM: Summary for Policymakers

TS: Technical Summary

ES: Executive Summary of a Chapter

Numbers denote the specific Chapters and Sections of a report.

For example, \{WGI TS.3; WGII 4.ES, Figure 4.3; WGIII Table 11.3\} would refer to the Technical Summary Section 3 of WGI, the Executive Summary and Figure 4.3 in Chapter 4 of WGII, and Table 11.3 in Chapter 11 of WGIII.

Other reports cited in this Synthesis Report:

TAR: Third Assessment Report

SROC: Special Report on Safeguarding the Ozone Layer and the Global Climate System 


\section{Climate Change 2007: Synthesis Report}

\section{Summary for Policymakers}

\section{An Assessment of the Intergovernmental Panel on Climate Change}

This summary, approved in detail at IPCC Plenary XXVII (Valencia, Spain, 12-17 November 2007), represents the formally agreed statement of the IPCC concerning key findings and uncertainties contained in the Working Group contributions to the Fourth Assessment Report.

\section{Based on a draft prepared by:}

Lenny Bernstein, Peter Bosch, Osvaldo Canziani, Zhenlin Chen, Renate Christ, Ogunlade Davidson, William Hare, Saleemul Huq, David Karoly, Vladimir Kattsov, Zbigniew Kundzewicz, Jian Liu, Ulrike Lohmann, Martin Manning, Taroh Matsuno, Bettina Menne, Bert Metz, Monirul Mirza, Neville Nicholls, Leonard Nurse, Rajendra Pachauri, Jean Palutikof, Martin Parry, Dahe Qin, Nijavalli Ravindranath, Andy Reisinger, Jiawen Ren, Keywan Riahi, Cynthia Rosenzweig, Matilde Rusticucci, Stephen Schneider, Youba Sokona, Susan Solomon, Peter Stott, Ronald Stouffer, Taishi Sugiyama, Rob Swart, Dennis Tirpak, Coleen Vogel, Gary Yohe 


\section{Introduction}

This Synthesis Report is based on the assessment carried out by the three Working Groups of the Intergovernmental Panel on Climate Change (IPCC). It provides an integrated view of climate change as the final part of the IPCC's Fourth Assessment Report (AR4).

A complete elaboration of the Topics covered in this summary can be found in this Synthesis Report and in the underlying reports of the three Working Groups.

\section{Observed changes in climate and their effects}

Warming of the climate system is unequivocal, as is now evident from observations of increases in global average air and ocean temperatures, widespread melting of snow and ice and rising global average sea level (Figure SPM.1). \{1.1\}

Eleven of the last twelve years (1995-2006) rank among the twelve warmest years in the instrumental record of global surface temperature (since 1850). The 100-year linear trend (1906-2005) of 0.74 [0.56 to 0.92$]^{\circ} \mathrm{C}^{1}$ is larger than the corresponding trend of 0.6 [0.4 to 0.8$]^{\circ} \mathrm{C}(1901-2000)$ given in the Third Assessment Report (TAR) (Figure SPM.1). The temperature increase is widespread over the globe and is greater at higher northern latitudes. Land regions have warmed faster than the oceans (Figures SPM.2, SPM.4). \{1.1, 1.2\}

Rising sea level is consistent with warming (Figure SPM.1). Global average sea level has risen since 1961 at an average rate of 1.8 [1.3 to 2.3$] \mathrm{mm} / \mathrm{yr}$ and since 1993 at 3.1 [2.4 to 3.8 ] mm/yr, with contributions from thermal expansion, melting glaciers and ice caps, and the polar ice sheets. Whether the faster rate for 1993 to 2003 reflects decadal variation or an increase in the longer-term trend is unclear. $\{1.1\}$

Observed decreases in snow and ice extent are also consistent with warming (Figure SPM.1). Satellite data since 1978 show that annual average Arctic sea ice extent has shrunk by 2.7 [2.1 to 3.3$] \%$ per decade, with larger decreases in summer of 7.4 [5.0 to 9.8 ]\% per decade. Mountain glaciers and snow cover on average have declined in both hemispheres. \{1.1\}

From 1900 to 2005, precipitation increased significantly in eastern parts of North and South America, northern Europe and northern and central Asia but declined in the Sahel, the
Mediterranean, southern Africa and parts of southern Asia. Globally, the area affected by drought has likely ${ }^{2}$ increased since the 1970s. \{1.1\}

It is very likely that over the past 50 years: cold days, cold nights and frosts have become less frequent over most land areas, and hot days and hot nights have become more frequent. It is likely that: heat waves have become more frequent over most land areas, the frequency of heavy precipitation events has increased over most areas, and since 1975 the incidence of extreme high sea level ${ }^{3}$ has increased worldwide. $\{1.1\}$

There is observational evidence of an increase in intense tropical cyclone activity in the North Atlantic since about 1970, with limited evidence of increases elsewhere. There is no clear trend in the annual numbers of tropical cyclones. It is difficult to ascertain longer-term trends in cyclone activity, particularly prior to 1970 . $\{1.1\}$

Average Northern Hemisphere temperatures during the second half of the $20^{\text {th }}$ century were very likely higher than during any other 50-year period in the last 500 years and likely the highest in at least the past 1300 years. $\{1.1\}$

Observational evidence ${ }^{4}$ from all continents and most oceans shows that many natural systems are being affected by regional climate changes, particularly temperature increases. $\{1.2\}$

Changes in snow, ice and frozen ground have with high confidence increased the number and size of glacial lakes, increased ground instability in mountain and other permafrost regions and led to changes in some Arctic and Antarctic ecosystems. \{1.2\}

There is high confidence that some hydrological systems have also been affected through increased runoff and earlier spring peak discharge in many glacier- and snow-fed rivers and through effects on thermal structure and water quality of warming rivers and lakes. $\{1.2\}$

In terrestrial ecosystems, earlier timing of spring events and poleward and upward shifts in plant and animal ranges are with very high confidence linked to recent warming. In some marine and freshwater systems, shifts in ranges and changes in algal, plankton and fish abundance are with high confidence associated with rising water temperatures, as well as related changes in ice cover, salinity, oxygen levels and circulation. $\{1.2\}$

Of the more than 29,000 observational data series, from 75 studies, that show significant change in many physical and biological systems, more than $89 \%$ are consistent with the direction of change expected as a response to warming (Fig-

\footnotetext{
${ }^{1}$ Numbers in square brackets indicate a $90 \%$ uncertainty interval around a best estimate, i.e. there is an estimated $5 \%$ likelihood that the value could be above the range given in square brackets and $5 \%$ likelihood that the value could be below that range. Uncertainty intervals are not necessarily symmetric around the corresponding best estimate.

${ }^{2}$ Words in italics represent calibrated expressions of uncertainty and confidence. Relevant terms are explained in the Box 'Treatment of uncertainty' in the Introduction of this Synthesis Report.

${ }^{3}$ Excluding tsunamis, which are not due to climate change. Extreme high sea level depends on average sea level and on regional weather systems. It is defined here as the highest $1 \%$ of hourly values of observed sea level at a station for a given reference period.

${ }^{4}$ Based largely on data sets that cover the period since 1970.
} 


\section{Changes in temperature, sea level and Northern Hemisphere snow cover}

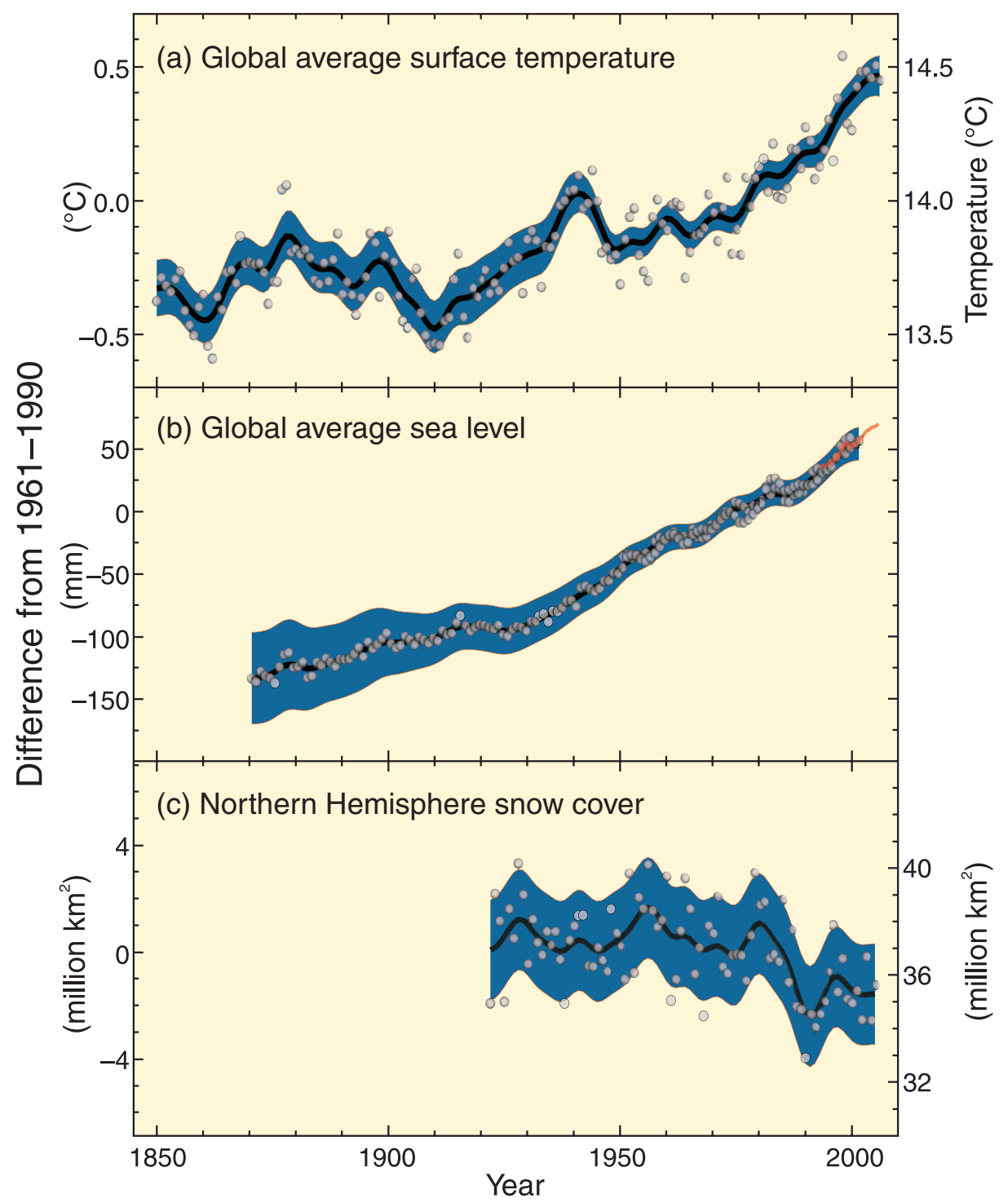

Figure SPM.1. Observed changes in (a) global average surface temperature; (b) global average sea level from tide gauge (blue) and satellite (red) data and (c) Northern Hemisphere snow cover for March-April. All differences are relative to corresponding averages for the period 19611990. Smoothed curves represent decadal averaged values while circles show yearly values. The shaded areas are the uncertainty intervals estimated from a comprehensive analysis of known uncertainties ( $a$ and $b$ ) and from the time series (c). \{Figure 1.1\}

ure SPM.2). However, there is a notable lack of geographic balance in data and literature on observed changes, with marked scarcity in developing countries. $\{1.2,1.3\}$

There is medium confidence that other effects of regional climate change on natural and human environments are emerging, although many are difficult to discern due to adaptation and non-climatic drivers. $\{1.2\}$

They include effects of temperature increases on: $\{1.2\}$

- agricultural and forestry management at Northern Hemisphere higher latitudes, such as earlier spring planting of crops, and alterations in disturbance regimes of forests due to fires and pests

- some aspects of human health, such as heat-related mortality in Europe, changes in infectious disease vectors in some areas, and allergenic pollen in Northern Hemisphere high and mid-latitudes

- some human activities in the Arctic (e.g. hunting and travel over snow and ice) and in lower-elevation alpine areas (such as mountain sports). 


\section{Changes in physical and biological systems and surface temperature 1970-2004}

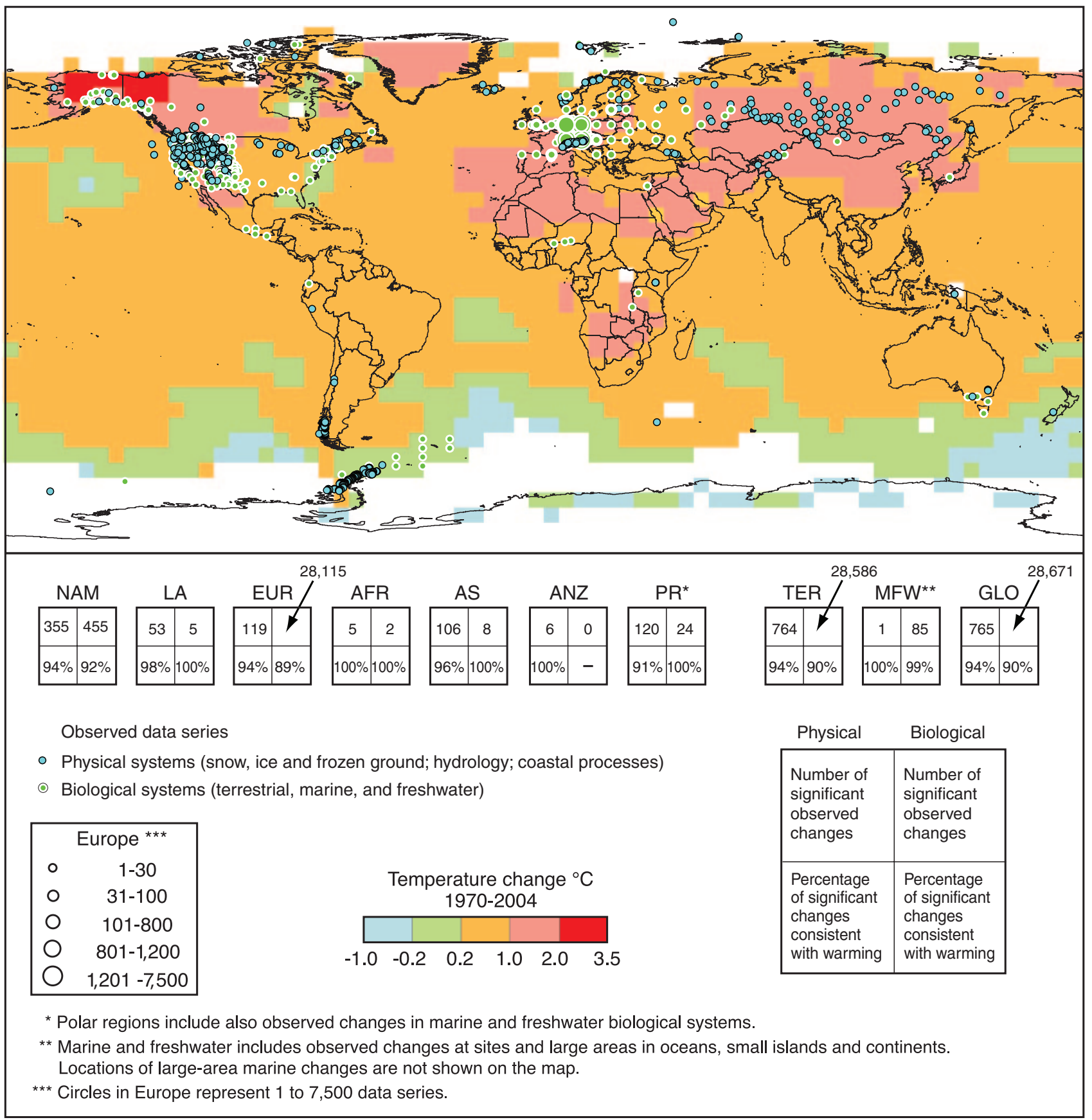

Figure SPM.2. Locations of significant changes in data series of physical systems (snow, ice and frozen ground; hydrology; and coastal processes) and biological systems (terrestrial, marine and freshwater biological systems), are shown together with surface air temperature changes over the period 1970-2004. A subset of about 29,000 data series was selected from about 80,000 data series from 577 studies. These met the following criteria: (1) ending in 1990 or later; (2) spanning a period of at least 20 years; and (3) showing a significant change in either direction, as assessed in individual studies. These data series are from about 75 studies (of which about 70 are new since the TAR) and contain about 29,000 data series, of which about 28,000 are from European studies. White areas do not contain sufficient observational climate data to estimate a temperature trend. The $2 \times 2$ boxes show the total number of data series with significant changes (top row) and the percentage of those consistent with warming (bottom row) for (i) continental regions: North America (NAM), Latin America (LA), Europe (EUR), Africa (AFR), Asia (AS), Australia and New Zealand (ANZ), and Polar Regions (PR) and (ii) global-scale: Terrestrial (TER), Marine and Freshwater (MFW), and Global (GLO). The numbers of studies from the seven regional boxes (NAM, EUR, AFR, AS, ANZ, PR) do not add up to the global (GLO) totals because numbers from regions except Polar do not include the numbers related to Marine and Freshwater (MFW) systems. Locations of largearea marine changes are not shown on the map. \{Figure 1.2\} 


\section{Causes of change}

Changes in atmospheric concentrations of greenhouse gases (GHGs) and aerosols, land cover and solar radiation alter the energy balance of the climate system. $\{2.2\}$

Global GHG emissions due to human activities have grown since pre-industrial times, with an increase of $70 \%$ between 1970 and 2004 (Figure SPM.3). ${ }^{5}\{2.1\}$

Carbon dioxide $\left(\mathrm{CO}_{2}\right)$ is the most important anthropogenic GHG. Its annual emissions grew by about $80 \%$ between 1970 and 2004. The long-term trend of declining $\mathrm{CO}_{2}$ emissions per unit of energy supplied reversed after 2000. $\{2.1\}$

Global atmospheric concentrations of $\mathrm{CO}_{2}$, methane $\left(\mathrm{CH}_{4}\right)$ and nitrous oxide $\left(\mathrm{N}_{2} \mathrm{O}\right)$ have increased markedly as a result of human activities since 1750 and now far exceed pre-industrial values determined from ice cores spanning many thousands of years. $\{2.2\}$

Atmospheric concentrations of $\mathrm{CO}_{2}(379 \mathrm{ppm})$ and $\mathrm{CH}_{4}$ $(1774 \mathrm{ppb})$ in 2005 exceed by far the natural range over the last 650,000 years. Global increases in $\mathrm{CO}_{2}$ concentrations are due primarily to fossil fuel use, with land-use change providing another significant but smaller contribution. It is very likely that the observed increase in $\mathrm{CH}_{4}$ concentration is predominantly due to agriculture and fossil fuel use. $\mathrm{CH}_{4}$ growth rates have declined since the early 1990s, consistent with total emissions (sum of anthropogenic and natural sources) being nearly constant during this period. The increase in $\mathrm{N}_{2} \mathrm{O}$ concentration is primarily due to agriculture. $\{2.2\}$

There is very high confidence that the net effect of human activities since 1750 has been one of warming. ${ }^{6}\{2.2\}$

Most of the observed increase in global average temperatures since the mid-20 ${ }^{\text {th }}$ century is very likely due to the observed increase in anthropogenic GHG concentrations. ${ }^{7}$ It is likely that there has been significant anthropogenic warming over the past $\mathbf{5 0}$ years averaged over each continent (except Antarctica) (Figure SPM.4). \{2.4\}

During the past 50 years, the sum of solar and volcanic forcings would likely have produced cooling. Observed patterns of warming and their changes are simulated only by models that include anthropogenic forcings. Difficulties remain in simulating and attributing observed temperature changes at smaller than continental scales. $\{2.4\}$

\section{Global anthropogenic GHG emissions}

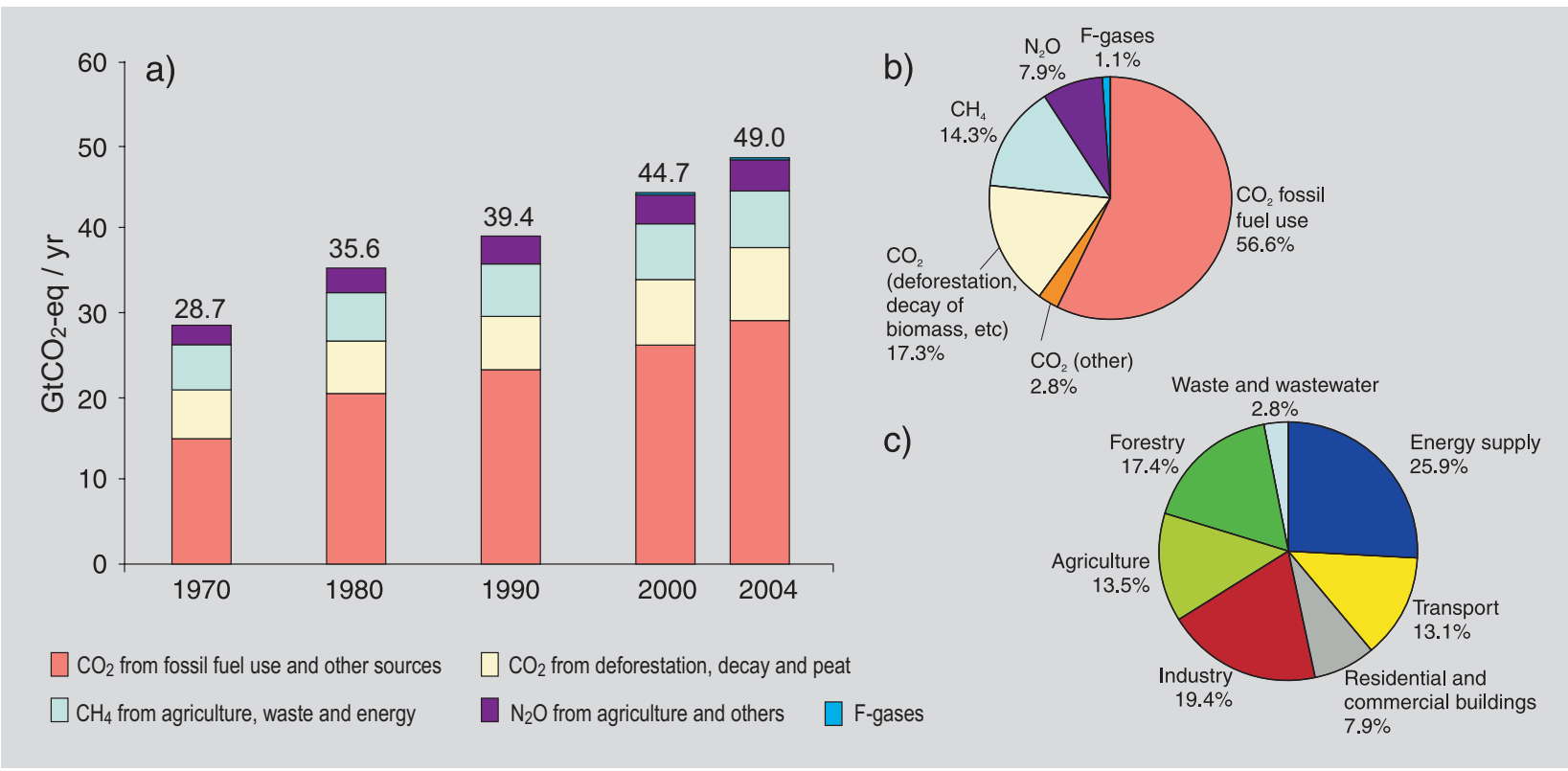

Figure SPM.3. (a) Global annual emissions of anthropogenic GHGs from 1970 to $2004 .^{5}$ (b) Share of different anthropogenic GHGs in total emissions in 2004 in terms of carbon dioxide equivalents $\left(\mathrm{CO}_{2}\right.$-eq). (c) Share of different sectors in total anthropogenic GHG emissions in 2004 in terms of $\mathrm{CO}_{2}$-eq. (Forestry includes deforestation.) \{Figure 2.1\}

${ }_{5}^{5}$ Includes only carbon dioxide $\left(\mathrm{CO}_{2}\right)$, methane $\left(\mathrm{CH}_{4}\right)$, nitrous oxide $\left(\mathrm{N}_{2} \mathrm{O}\right)$, hydrofluorocarbons (HFCs), perfluorocarbons (PFCs) and sulphurhexafluoride $\left(\mathrm{SF}_{6}\right)$, whose emissions are covered by the United Nations Framework Convention on Climate Change (UNFCCC). These GHGs are weighted by their 100-year Global Warming Potentials, using values consistent with reporting under the UNFCCC.

${ }^{6}$ Increases in GHGs tend to warm the surface while the net effect of increases in aerosols tends to cool it. The net effect due to human activities since the pre-industrial era is one of warming $\left(+1.6[+0.6\right.$ to +2.4$\left.] \mathrm{W} / \mathrm{m}^{2}\right)$. In comparison, changes in solar irradiance are estimated to have caused a small warming effect $\left(+0.12[+0.06\right.$ to +0.30$\left.] \mathrm{W} / \mathrm{m}^{2}\right)$.

${ }^{7}$ Consideration of remaining uncertainty is based on current methodologies. 


\section{Global and continental temperature change}

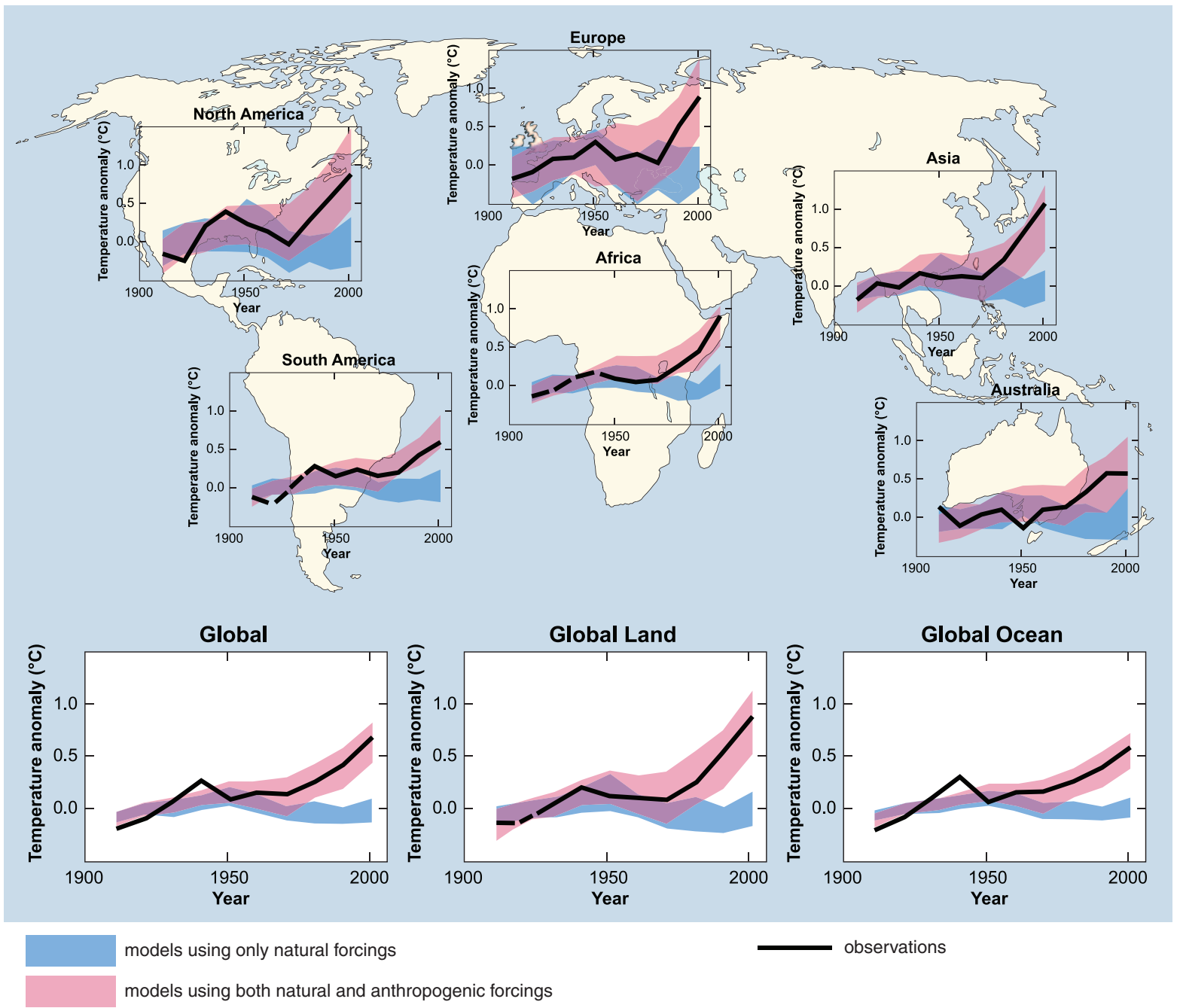

Figure SPM.4. Comparison of observed continental- and global-scale changes in surface temperature with results simulated by climate models using either natural or both natural and anthropogenic forcings. Decadal averages of observations are shown for the period 1906-2005 (black line) plotted against the centre of the decade and relative to the corresponding average for the period 1901-1950. Lines are dashed where spatial coverage is less than $50 \%$. Blue shaded bands show the 5 to $95 \%$ range for 19 simulations from five climate models using only the natural forcings due to solar activity and volcanoes. Red shaded bands show the 5 to $95 \%$ range for 58 simulations from 14 climate models using both natural and anthropogenic forcings. \{Figure 2.5\}

\section{Advances since the TAR show that discernible human influences extend beyond average temperature to other aspects of climate. $\{2.4\}$}

Human influences have: $\{2.4\}$

- very likely contributed to sea level rise during the latter half of the $20^{\text {th }}$ century

- likely contributed to changes in wind patterns, affecting extra-tropical storm tracks and temperature patterns

- likely increased temperatures of extreme hot nights, cold nights and cold days

- more likely than not increased risk of heat waves, area affected by drought since the 1970s and frequency of heavy precipitation events.
Anthropogenic warming over the last three decades has likely had a discernible influence at the global scale on observed changes in many physical and biological systems. $\{2.4\}$

Spatial agreement between regions of significant warming across the globe and locations of significant observed changes in many systems consistent with warming is very unlikely to be due solely to natural variability. Several modelling studies have linked some specific responses in physical and biological systems to anthropogenic warming. \{2.4\}

More complete attribution of observed natural system responses to anthropogenic warming is currently prevented by the short time scales of many impact studies, greater natural climate variability at regional scales, contributions of nonclimate factors and limited spatial coverage of studies. $\{2.4\}$ 


\section{Projected climate change and its impacts}

There is high agreement and much evidence that with current climate change mitigation policies and related sustainable development practices, global GHG emissions will continue to grow over the next few decades. $\{3.1\}$

The IPCC Special Report on Emissions Scenarios (SRES, 2000) projects an increase of global GHG emissions by 25 to 90\% ( $\mathrm{CO}_{2}$-eq) between 2000 and 2030 (Figure SPM.5), with fossil fuels maintaining their dominant position in the global energy mix to 2030 and beyond. More recent scenarios without additional emissions mitigation are comparable in range. ${ }^{8,9}\{3.1\}$

Continued GHG emissions at or above current rates would cause further warming and induce many changes in the global climate system during the $21^{\text {st }}$ century that would very likely be larger than those observed during the $20^{\text {th }}$ century (Table SPM.1, Figure SPM.5). $\{3.2 .1\}$
For the next two decades a warming of about $0.2^{\circ} \mathrm{C}$ per decade is projected for a range of SRES emissions scenarios. Even if the concentrations of all GHGs and aerosols had been kept constant at year 2000 levels, a further warming of about $0.1^{\circ} \mathrm{C}$ per decade would be expected. Afterwards, temperature projections increasingly depend on specific emissions scenarios. $\{3.2\}$

The range of projections (Table SPM.1) is broadly consistent with the TAR, but uncertainties and upper ranges for temperature are larger mainly because the broader range of available models suggests stronger climate-carbon cycle feedbacks. Warming reduces terrestrial and ocean uptake of atmospheric $\mathrm{CO}_{2}$, increasing the fraction of anthropogenic emissions remaining in the atmosphere. The strength of this feedback effect varies markedly among models. $\{2.3,3.2 .1\}$

Because understanding of some important effects driving sea level rise is too limited, this report does not assess the likelihood, nor provide a best estimate or an upper bound for sea level rise. Table SPM.1 shows model-based projections

\section{Scenarios for GHG emissions from 2000 to 2100 (in the absence of additional climate policies)} and projections of surface temperatures
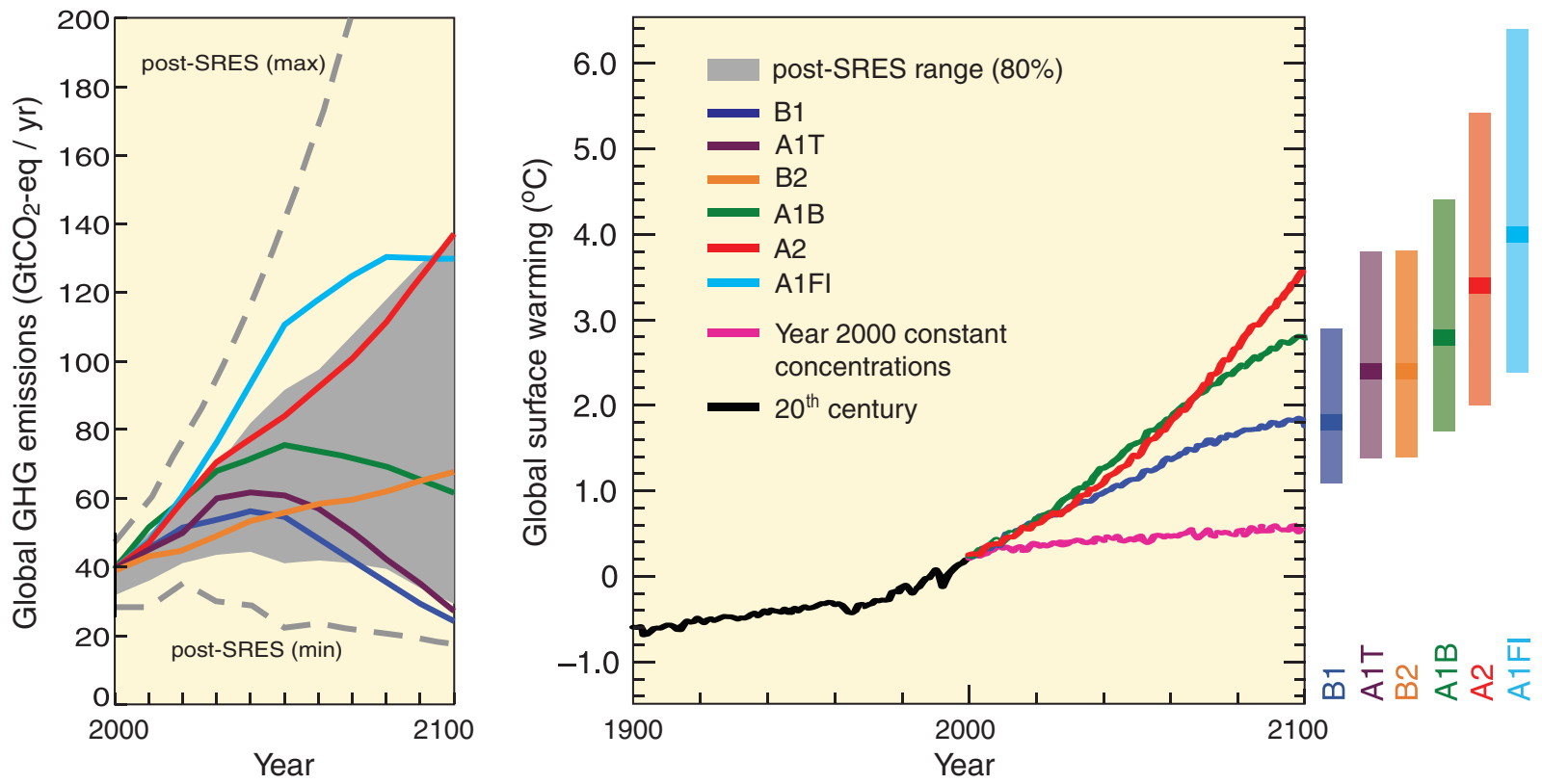

Figure SPM.5. Left Panel: Global GHG emissions (in $\mathrm{GtCO}_{2}$-eq) in the absence of climate policies: six illustrative SRES marker scenarios (coloured lines) and the $80^{\text {th }}$ percentile range of recent scenarios published since SRES (post-SRES) (gray shaded area). Dashed lines show the full range of post-SRES scenarios. The emissions include $\mathrm{CO}_{2}, \mathrm{CH}_{4}, \mathrm{~N}_{2} \mathrm{O}$ and F-gases. Right Panel: Solid lines are multi-model global averages of surface warming for scenarios $A 2, A 1 B$ and $B 1$, shown as continuations of the $20^{\text {th }}$-century simulations. These projections also take into account emissions of short-lived GHGs and aerosols. The pink line is not a scenario, but is for Atmosphere-Ocean General Circulation Model (AOGCM) simulations where atmospheric concentrations are held constant at year 2000 values. The bars at the right of the figure indicate the best estimate (solid line within each bar) and the likely range assessed for the six SRES marker scenarios at 2090-2099. All temperatures are relative to the period 1980-1999. \{Figures 3.1 and 3.2\}

${ }^{8}$ For an explanation of SRES emissions scenarios, see Box 'SRES scenarios' in Topic 3 of this Synthesis Report. These scenarios do not include additional climate policies above current ones; more recent studies differ with respect to UNFCCC and Kyoto Protocol inclusion.

${ }^{9}$ Emission pathways of mitigation scenarios are discussed in Section 5. 
Table SPM.1. Projected global average surface warming and sea level rise at the end of the $21^{\text {st }}$ century. $\{$ Table 3.1$\}$

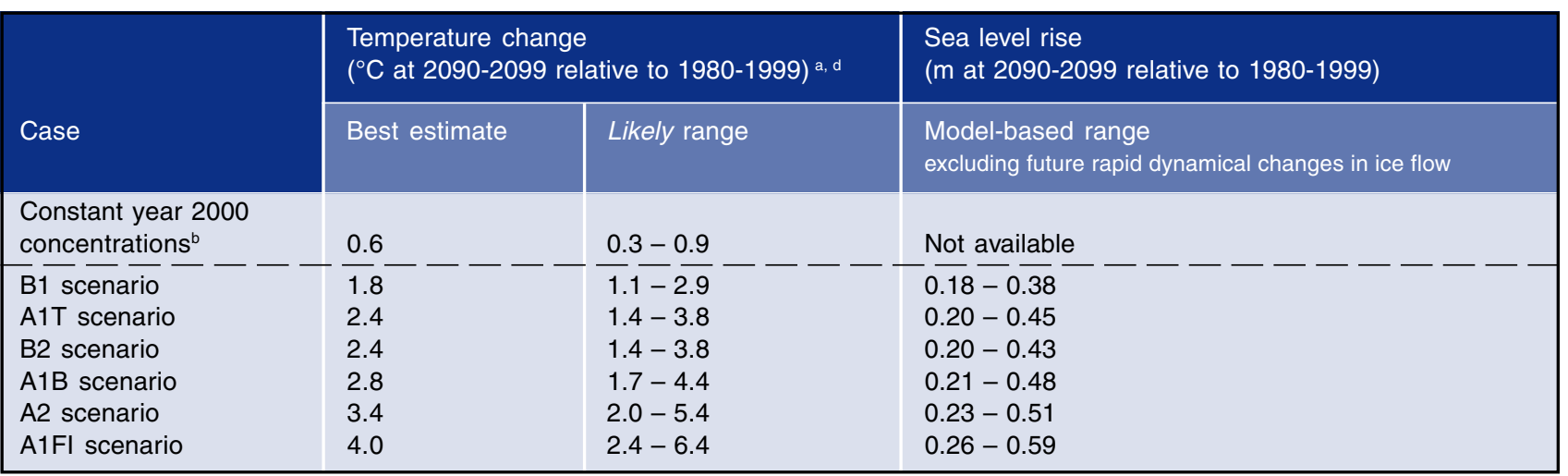

Notes:

a) Temperatures are assessed best estimates and likely uncertainty ranges from a hierarchy of models of varying complexity as well as observational constraints.

b) Year 2000 constant composition is derived from Atmosphere-Ocean General Circulation Models (AOGCMs) only.

c) All scenarios above are six SRES marker scenarios. Approximate $\mathrm{CO}_{2}$-eq concentrations corresponding to the computed radiative forcing due to anthropogenic GHGs and aerosols in 2100 (see p. 823 of the Working Group I TAR) for the SRES B1, AIT, B2, A1B, A2 and A1FI illustrative marker scenarios are about 600, 700, 800, 850, 1250 and 1550ppm, respectively.

d) Temperature changes are expressed as the difference from the period 1980-1999. To express the change relative to the period 18501899 add $0.5^{\circ} \mathrm{C}$.

of global average sea level rise for 2090-2099. ${ }^{10}$ The projections do not include uncertainties in climate-carbon cycle feedbacks nor the full effects of changes in ice sheet flow, therefore the upper values of the ranges are not to be considered upper bounds for sea level rise. They include a contribution from increased Greenland and Antarctic ice flow at the rates observed for 1993-2003, but this could increase or decrease in the future. ${ }^{11}\{3.2 .1\}$

There is now higher confidence than in the TAR in projected patterns of warming and other regional-scale features, including changes in wind patterns, precipitation and some aspects of extremes and sea ice. \{3.2.2\}

Regional-scale changes include: $\{3.2 .2\}$

- warming greatest over land and at most high northern latitudes and least over Southern Ocean and parts of the North Atlantic Ocean, continuing recent observed trends (Figure SPM.6)

- contraction of snow cover area, increases in thaw depth over most permafrost regions and decrease in sea ice extent; in some projections using SRES scenarios, Arctic late-summer sea ice disappears almost entirely by the latter part of the $21^{\text {st }}$ century

- very likely increase in frequency of hot extremes, heat waves and heavy precipitation

- likely increase in tropical cyclone intensity; less confidence in global decrease of tropical cyclone numbers
- poleward shift of extra-tropical storm tracks with consequent changes in wind, precipitation and temperature patterns

- very likely precipitation increases in high latitudes and likely decreases in most subtropical land regions, continuing observed recent trends.

There is high confidence that by mid-century, annual river runoff and water availability are projected to increase at high latitudes (and in some tropical wet areas) and decrease in some dry regions in the mid-latitudes and tropics. There is also high confidence that many semi-arid areas (e.g. Mediterranean Basin, western United States, southern Africa and north-eastern Brazil) will suffer a decrease in water resources due to climate change. \{3.3.1, Figure 3.5$\}$

\section{Studies since the TAR have enabled more systematic understanding of the timing and magnitude of impacts related to differing amounts and rates of climate change. $\{3.3 .1,3.3 .2\}$}

Figure SPM.7 presents examples of this new information for systems and sectors. The top panel shows impacts increasing with increasing temperature change. Their estimated magnitude and timing is also affected by development pathway (lower panel). \{3.3.1\}

Examples of some projected impacts for different regions are given in Table SPM.2.

\footnotetext{
${ }^{10}$ TAR projections were made for 2100, whereas the projections for this report are for 2090-2099. The TAR would have had similar ranges to those in Table SPM. 1 if it had treated uncertainties in the same way.

${ }^{11}$ For discussion of the longer term, see material below.
} 


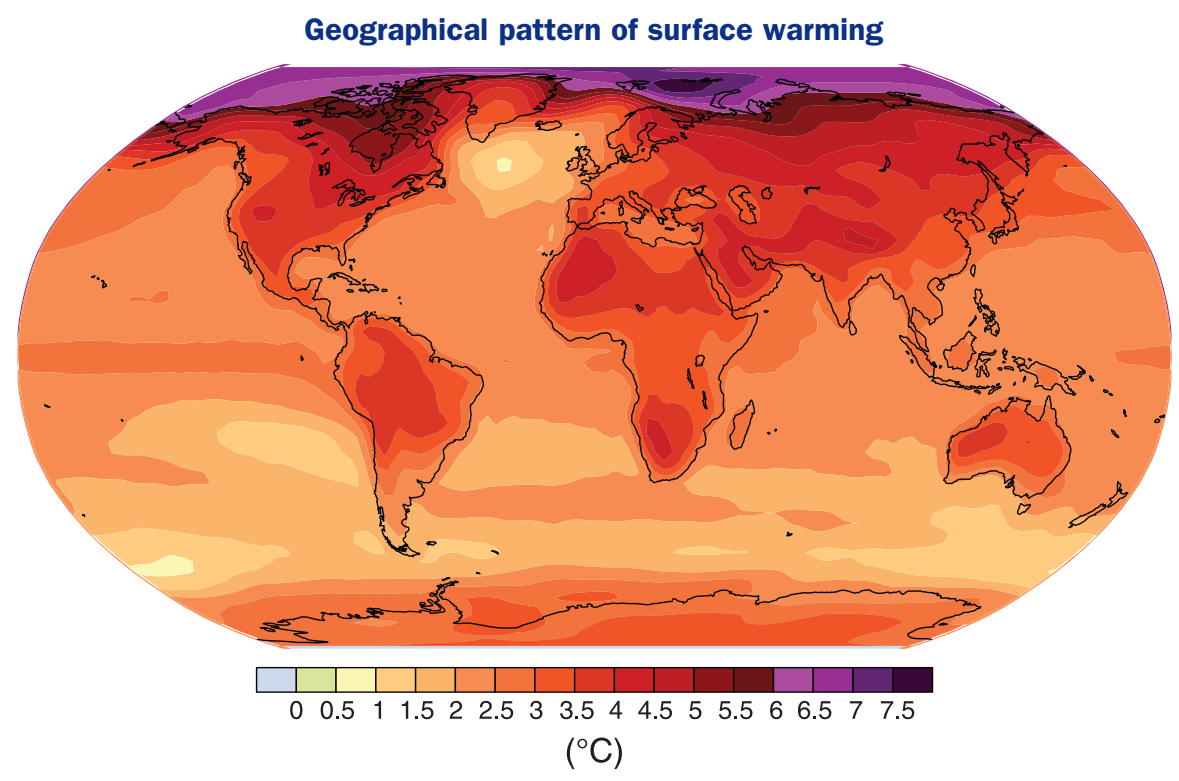

Figure SPM.6. Projected surface temperature changes for the late $21^{\text {st }}$ century (2090-2099). The map shows the multi-AOGCM average projection for the A1B SRES scenario. Temperatures are relative to the period 1980-1999. \{Figure 3.2\}

Some systems, sectors and regions are likely to be especially affected by climate change. ${ }^{12}\{3.3 .3\}$

Systems and sectors: $\{3.3 .3\}$

- particular ecosystems:

- terrestrial: tundra, boreal forest and mountain regions because of sensitivity to warming; mediterranean-type ecosystems because of reduction in rainfall; and tropical rainforests where precipitation declines

- coastal: mangroves and salt marshes, due to multiple stresses

- marine: coral reefs due to multiple stresses; the sea ice biome because of sensitivity to warming

- water resources in some dry regions at mid-latitudes ${ }^{13}$ and in the dry tropics, due to changes in rainfall and evapotranspiration, and in areas dependent on snow and ice melt

- agriculture in low latitudes, due to reduced water availability

- low-lying coastal systems, due to threat of sea level rise and increased risk from extreme weather events

- human health in populations with low adaptive capacity.

Regions: $\{3.3 .3\}$

- the Arctic, because of the impacts of high rates of projected warming on natural systems and human communities
- Africa, because of low adaptive capacity and projected climate change impacts

- small islands, where there is high exposure of population and infrastructure to projected climate change impacts

- Asian and African megadeltas, due to large populations and high exposure to sea level rise, storm surges and river flooding.

Within other areas, even those with high incomes, some people (such as the poor, young children and the elderly) can be particularly at risk, and also some areas and some activities. $\{3.3 .3\}$

\section{Ocean acidification}

The uptake of anthropogenic carbon since 1750 has led to the ocean becoming more acidic with an average decrease in $\mathrm{pH}$ of 0.1 units. Increasing atmospheric $\mathrm{CO}_{2}$ concentrations lead to further acidification. Projections based on SRES scenarios give a reduction in average global surface ocean $\mathrm{pH}$ of between 0.14 and 0.35 units over the $21^{\text {st }}$ century. While the effects of observed ocean acidification on the marine biosphere are as yet undocumented, the progressive acidification of oceans is expected to have negative impacts on marine shell-forming organisms (e.g. corals) and their dependent species. $\{3.3 .4\}$

\footnotetext{
12 Identified on the basis of expert judgement of the assessed literature and considering the magnitude, timing and projected rate of climate change, sensitivity and adaptive capacity.

${ }^{13}$ Including arid and semi-arid regions.
} 
Examples of impacts associated with global average temperature change (Impacts will vary by extent of adaptation, rate of temperature change and socio-economic pathway)

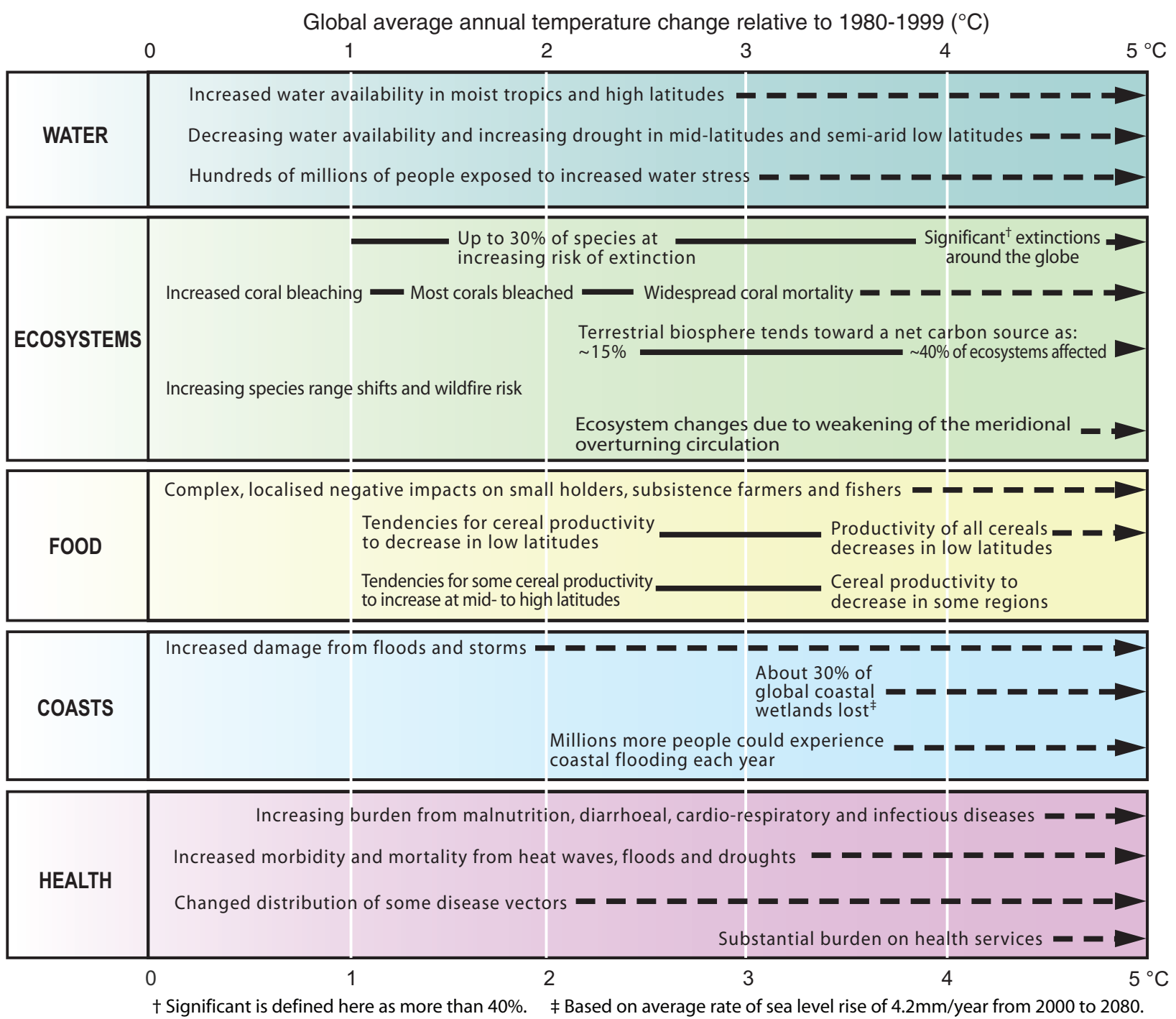

Warming by 2090-2099 relative to 1980-1999 for non-mitigation scenarios

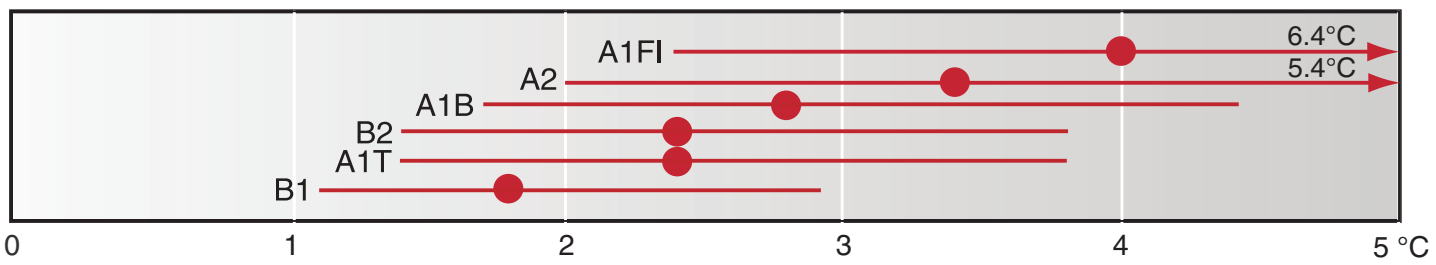

Figure SPM.7. Examples of impacts associated with projected global average surface warming. Upper panel: Illustrative examples of global impacts projected for climate changes (and sea level and atmospheric $\mathrm{CO}_{2}$ where relevant) associated with different amounts of increase in global average surface temperature in the $21^{\text {st }}$ century. The black lines link impacts; broken-line arrows indicate impacts continuing with increasing temperature. Entries are placed so that the left-hand side of text indicates the approximate level of warming that is associated with the onset of a given impact. Quantitative entries for water scarcity and flooding represent the additional impacts of climate change relative to the conditions projected across the range of SRES scenarios A1FI, A2, B1 and B2. Adaptation to climate change is not included in these estimations. Confidence levels for all statements are high. Lower panel: Dots and bars indicate the best estimate and likely ranges of warming assessed for the six SRES marker scenarios for 2090-2099 relative to 1980-1999. \{Figure 3.6\} 
Table SPM.2. Examples of some projected regional impacts. \{3.3.2\}

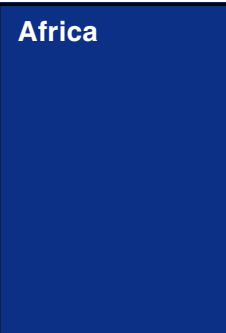

Asia

Australia and
New Zealand

New Zealand

.

- By 2020 , between 75 and 250 million of people are projected to be exposed to increased water stress due to climate change.

- By 2020 , in some countries, yields from rain-fed agriculture could be reduced by up to $50 \%$. Agricultural production, including access to food, in many African countries is projected to be severely compromised. This would further adversely affect food security and exacerbate malnutrition.

- Towards the end of the $21^{\text {st }}$ century, projected sea level rise will affect low-lying coastal areas with large populations. The cost of adaptation could amount to at least 5 to $10 \%$ of Gross Domestic Product (GDP).

- By 2080 , an increase of 5 to $8 \%$ of arid and semi-arid land in Africa is projected under a range of climate scenarios (TS).

- By the 2050s, freshwater availability in Central, South, East and South-East Asia, particularly in large river basins, is projected to decrease.

- Coastal areas, especially heavily populated megadelta regions in South, East and South-East Asia, will be at greatest risk due to increased flooding from the sea and, in some megadeltas, flooding from the rivers.

- Climate change is projected to compound the pressures on natural resources and the environment associated with rapid urbanisation, industrialisation and economic development.

- Endemic morbidity and mortality due to diarrhoeal disease primarily associated with floods and droughts are expected to rise in East, South and South-East Asia due to projected changes in the hydrological cycle.

- By 2020, significant loss of biodiversity is projected to occur in some ecologically rich sites, including the Great Barrier Reef and Queensland Wet Tropics.

- By 2030, water security problems are projected to intensify in southern and eastern Australia and, in New Zealand, in Northland and some eastern regions.

- By 2030, production from agriculture and forestry is projected to decline over much of southern and eastern Australia, and over parts of eastern New Zealand, due to increased drought and fire. However, in New Zealand, initial benefits are projected in some other regions.

- By 2050, ongoing coastal development and population growth in some areas of Australia and New Zealand are projected to exacerbate risks from sea level rise and increases in the severity and frequency of storms and coastal flooding.

Europe

- Climate change is expected to magnify regional differences in Europe's natural resources and assets. Negative impacts will include increased risk of inland flash floods and more frequent coastal flooding and increased erosion (due to storminess and sea level rise).

- Mountainous areas will face glacier retreat, reduced snow cover and winter tourism, and extensive species losses (in some areas up to $60 \%$ under high emissions scenarios by 2080).

- In southern Europe, climate change is projected to worsen conditions (high temperatures and drought) in a region already vulnerable to climate variability, and to reduce water availability, hydropower potential, summer tourism and, in general, crop productivity.

- Climate change is also projected to increase the health risks due to heat waves and the frequency of wildfires.

Latin America

- By mid-century, increases in temperature and associated decreases in soil water are projected to lead to gradual replacement of tropical forest by savanna in eastern Amazonia. Semi-arid vegetation will tend to be replaced by arid-land vegetation.

- There is a risk of significant biodiversity loss through species extinction in many areas of tropical Latin America.

- Productivity of some important crops is projected to decrease and livestock productivity to decline, with adverse consequences for food security. In temperate zones, soybean yields are projected to increase. Overall, the number of people at risk of hunger is projected to increase (TS; medium confidence).

- Changes in precipitation patterns and the disappearance of glaciers are projected to significantly affect water availability for human consumption, agriculture and energy generation.

North America

- Warming in western mountains is projected to cause decreased snowpack, more winter flooding and reduced summer flows, exacerbating competition for over-allocated water resources.

- In the early decades of the century, moderate climate change is projected to increase aggregate yields of rain-fed agriculture by 5 to $20 \%$, but with important variability among regions. Major challenges are projected for crops that are near the warm end of their suitable range or which depend on highly utilised water resources.

- Cities that currently experience heat waves are expected to be further challenged by an increased number, intensity and duration of heat waves during the course of the century, with potential for adverse health impacts.

- Coastal communities and habitats will be increasingly stressed by climate change impacts interacting with development and pollution. 
Table SPM.2. continued...

Polar Regions

- The main projected biophysical effects are reductions in thickness and extent of glaciers, ice sheets and sea ice, and changes in natural ecosystems with detrimental effects on many organisms including migratory birds, mammals and higher predators.

- For human communities in the Arctic, impacts, particularly those resulting from changing snow and ice conditions, are projected to be mixed.

- Detrimental impacts would include those on infrastructure and traditional indigenous ways of life.

- In both polar regions, specific ecosystems and habitats are projected to be vulnerable, as climatic barriers to species invasions are lowered.

Small Islands

- Sea level rise is expected to exacerbate inundation, storm surge, erosion and other coastal hazards, thus threatening vital infrastructure, settlements and facilities that support the livelihood of island communities.

- Deterioration in coastal conditions, for example through erosion of beaches and coral bleaching, is expected to affect local resources.

- By mid-century, climate change is expected to reduce water resources in many small islands, e.g. in the Caribbean and Pacific, to the point where they become insufficient to meet demand during low-rainfall periods.

- With higher temperatures, increased invasion by non-native species is expected to occur, particularly on mid- and high-latitude islands.

Note:

Unless stated explicitly, all entries are from Working Group II SPM text, and are either very high confidence or high confidence statements, reflecting different sectors (agriculture, ecosystems, water, coasts, health, industry and settlements). The Working Group II SPM refers to the source of the statements, timelines and temperatures. The magnitude and timing of impacts that will ultimately be realised will vary with the amount and rate of climate change, emissions scenarios, development pathways and adaptation.

\begin{abstract}
Altered frequencies and intensities of extreme weather, together with sea level rise, are expected to have mostly adverse effects on natural and human systems. \{3.3.5\}
\end{abstract}

Examples for selected extremes and sectors are shown in Table SPM.3.

Anthropogenic warming and sea level rise would continue for centuries due to the time scales associated with climate processes and feedbacks, even if GHG concentrations were to be stabilised. $\{3.2 .3\}$

Estimated long-term (multi-century) warming corresponding to the six AR4 Working Group III stabilisation categories is shown in Figure SPM.8.
Contraction of the Greenland ice sheet is projected to continue to contribute to sea level rise after 2100. Current models suggest virtually complete elimination of the Greenland ice sheet and a resulting contribution to sea level rise of about $7 \mathrm{~m}$ if global average warming were sustained for millennia in excess of 1.9 to $4.6^{\circ} \mathrm{C}$ relative to pre-industrial values. The corresponding future temperatures in Greenland are comparable to those inferred for the last interglacial period 125,000 years ago, when palaeoclimatic information suggests reductions of polar land ice extent and 4 to $6 \mathrm{~m}$ of sea level rise. $\{3.2 .3\}$

Current global model studies project that the Antarctic ice sheet will remain too cold for widespread surface melting and gain mass due to increased snowfall. However, net loss of ice mass could occur if dynamical ice discharge dominates the ice sheet mass balance. $\{3.2 .3\}$

Estimated multi-century warming relative to 1980-1999 for AR4 stabilisation categories

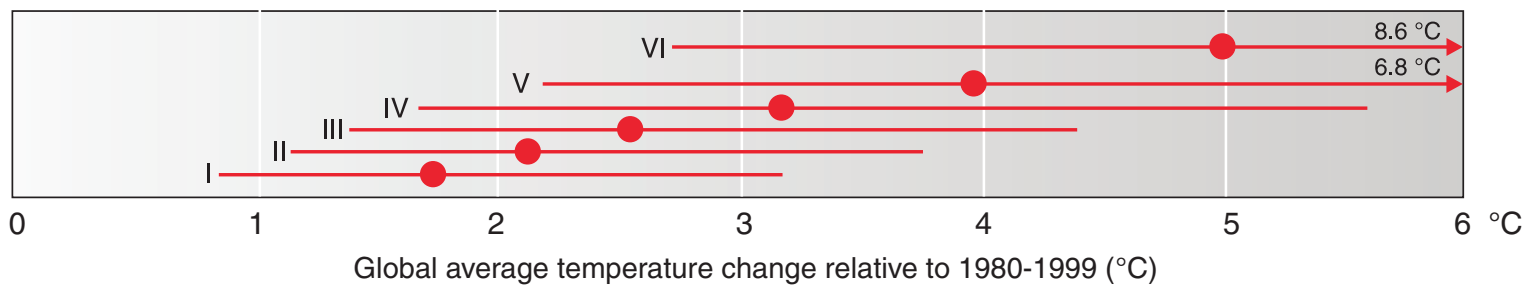

Figure SPM.8. Estimated long-term (multi-century) warming corresponding to the six AR4 Working Group III stabilisation categories (Table SPM.6). The temperature scale has been shifted by $-0.5^{\circ} \mathrm{C}$ compared to Table SPM. 6 to account approximately for the warming between preindustrial and 1980-1999. For most stabilisation levels global average temperature is approaching the equilibrium level over a few centuries. For

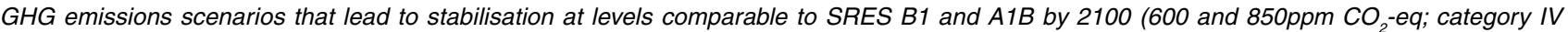
and $V$ ), assessed models project that about 65 to $70 \%$ of the estimated global equilibrium temperature increase, assuming a climate sensitivity of $3^{\circ} \mathrm{C}$, would be realised at the time of stabilisation. For the much lower stabilisation scenarios (category I and II, Figure SPM.11), the equilibrium temperature may be reached earlier. \{Figure 3.4$\}$ 
Table SPM.3. Examples of possible impacts of climate change due to changes in extreme weather and climate events, based on projections to the mid- to late $21^{\text {st }}$ century. These do not take into account any changes or developments in adaptive capacity. The likelihood estimates in column two relate to the phenomena listed in column one. \{Table 3.2\}

\begin{tabular}{|c|c|c|c|c|c|}
\hline \multirow{2}{*}{$\begin{array}{l}\text { Phenomenon }{ }^{\mathrm{a}} \text { and } \\
\text { direction of trend }\end{array}$} & \multirow{2}{*}{$\begin{array}{l}\text { Likelihood of } \\
\text { future trends } \\
\text { based on } \\
\text { projections } \\
\text { for } 21^{\text {st }} \text { century } \\
\text { using SRES } \\
\text { scenarios }\end{array}$} & \multicolumn{4}{|c|}{ Examples of major projected impacts by sector } \\
\hline & & $\begin{array}{l}\text { Agriculture, forestry } \\
\text { and ecosystems }\end{array}$ & Water resources & Human health & $\begin{array}{l}\text { Industry, settlement } \\
\text { and society }\end{array}$ \\
\hline $\begin{array}{l}\text { Over most land } \\
\text { areas, warmer and } \\
\text { fewer cold days } \\
\text { and nights, warmer } \\
\text { and more frequent } \\
\text { hot days and nights }\end{array}$ & $\begin{array}{l}\text { Virtually } \\
\text { certain }^{\mathrm{b}}\end{array}$ & $\begin{array}{l}\text { Increased yields in } \\
\text { colder environments; } \\
\text { decreased yields in } \\
\text { warmer environments; } \\
\text { increased insect } \\
\text { outbreaks }\end{array}$ & $\begin{array}{l}\text { Effects on water } \\
\text { resources relying on } \\
\text { snowmelt; effects on } \\
\text { some water supplies }\end{array}$ & $\begin{array}{l}\text { Reduced human } \\
\text { mortality from } \\
\text { decreased cold } \\
\text { exposure }\end{array}$ & $\begin{array}{l}\text { Reduced energy demand for } \\
\text { heating; increased demand } \\
\text { for cooling; declining air quality } \\
\text { in cities; reduced disruption to } \\
\text { transport due to snow, ice; } \\
\text { effects on winter tourism }\end{array}$ \\
\hline $\begin{array}{l}\text { Warm spells/heat } \\
\text { waves. Frequency } \\
\text { increases over most } \\
\text { land areas }\end{array}$ & Very likely & $\begin{array}{l}\text { Reduced yields in } \\
\text { warmer regions } \\
\text { due to heat stress; } \\
\text { increased danger of } \\
\text { wildfire }\end{array}$ & $\begin{array}{l}\text { Increased water } \\
\text { demand; water } \\
\text { quality problems, } \\
\text { e.g. algal blooms }\end{array}$ & $\begin{array}{l}\text { Increased risk of } \\
\text { heat-related } \\
\text { mortality, especially } \\
\text { for the elderly, } \\
\text { chronically sick, } \\
\text { very young and } \\
\text { socially isolated }\end{array}$ & $\begin{array}{l}\text { Reduction in quality of life for } \\
\text { people in warm areas without } \\
\text { appropriate housing; impacts } \\
\text { on the elderly, very young and } \\
\text { poor }\end{array}$ \\
\hline $\begin{array}{l}\text { Heavy precipitation } \\
\text { events. Frequency } \\
\text { increases over most } \\
\text { areas }\end{array}$ & Very likely & $\begin{array}{l}\text { Damage to crops; } \\
\text { soil erosion, inability } \\
\text { to cultivate land due } \\
\text { to waterlogging of } \\
\text { soils }\end{array}$ & $\begin{array}{l}\text { Adverse effects on } \\
\text { quality of surface } \\
\text { and groundwater; } \\
\text { contamination of } \\
\text { water supply; water } \\
\text { scarcity may be } \\
\text { relieved }\end{array}$ & $\begin{array}{l}\text { Increased risk of } \\
\text { deaths, injuries and } \\
\text { infectious, respiratory } \\
\text { and skin diseases }\end{array}$ & $\begin{array}{l}\text { Disruption of settlements, } \\
\text { commerce, transport and } \\
\text { societies due to flooding: } \\
\text { pressures on urban and rural } \\
\text { infrastructures; loss of property }\end{array}$ \\
\hline $\begin{array}{l}\text { Area affected by } \\
\text { drought increases }\end{array}$ & Likely & $\begin{array}{l}\text { Land degradation; } \\
\text { lower yields/crop } \\
\text { damage and failure; } \\
\text { increased livestock } \\
\text { deaths; increased } \\
\text { risk of wildfire }\end{array}$ & $\begin{array}{l}\text { More widespread } \\
\text { water stress }\end{array}$ & $\begin{array}{l}\text { Increased risk of } \\
\text { food and water } \\
\text { shortage; increased } \\
\text { risk of malnutrition; } \\
\text { increased risk of } \\
\text { water- and food- } \\
\text { borne diseases }\end{array}$ & $\begin{array}{l}\text { Water shortage for settlements, } \\
\text { industry and societies; } \\
\text { reduced hydropower generation } \\
\text { potentials; potential for } \\
\text { population migration }\end{array}$ \\
\hline $\begin{array}{l}\text { Increased incidence } \\
\text { of extreme high } \\
\text { sea level (excludes } \\
\text { tsunamis) }\end{array}$ & Likely $^{\mathrm{d}}$ & $\begin{array}{l}\text { Salinisation of } \\
\text { irrigation water, } \\
\text { estuaries and fresh- } \\
\text { water systems }\end{array}$ & $\begin{array}{l}\text { Decreased fresh- } \\
\text { water availability due } \\
\text { to saltwater intrusion }\end{array}$ & $\begin{array}{l}\text { Increased risk of } \\
\text { deaths and injuries } \\
\text { by drowning in floods; } \\
\text { migration-related } \\
\text { health effects }\end{array}$ & $\begin{array}{l}\text { Costs of coastal protection } \\
\text { versus costs of land-use } \\
\text { relocation; potential for } \\
\text { movement of populations and } \\
\text { infrastructure; also see tropical } \\
\text { cyclones above }\end{array}$ \\
\hline
\end{tabular}

Notes:

a) See Working Group I Table 3.7 for further details regarding definitions.

b) Warming of the most extreme days and nights each year.

c) Extreme high sea level depends on average sea level and on regional weather systems. It is defined as the highest $1 \%$ of hourly values of observed sea level at a station for a given reference period.

d) In all scenarios, the projected global average sea level at 2100 is higher than in the reference period. The effect of changes in regional weather systems on sea level extremes has not been assessed.

\section{Anthropogenic warming could lead to some impacts that are abrupt or irreversible, depending upon the rate and magnitude of the climate change. $\{3.4\}$}

Partial loss of ice sheets on polar land could imply metres of sea level rise, major changes in coastlines and inundation of low-lying areas, with greatest effects in river deltas and low-lying islands. Such changes are projected to occur over millennial time scales, but more rapid sea level rise on century time scales cannot be excluded. $\{3.4\}$

Climate change is likely to lead to some irreversible impacts. There is medium confidence that approximately 20 to $30 \%$ of species assessed so far are likely to be at increased risk of extinction if increases in global average warming exceed 1.5 to $2.5^{\circ} \mathrm{C}$ (relative to $1980-1999$ ). As global average 
temperature increase exceeds about $3.5^{\circ} \mathrm{C}$, model projections suggest significant extinctions (40 to $70 \%$ of species assessed) around the globe. $\{3.4\}$

Based on current model simulations, the meridional overturning circulation (MOC) of the Atlantic Ocean will very likely slow down during the $21^{\text {st }}$ century; nevertheless temperatures over the Atlantic and Europe are projected to increase. The MOC is very unlikely to undergo a large abrupt transition during the $21^{\text {st }}$ century. Longer-term MOC changes cannot be assessed with confidence. Impacts of large-scale and persistent changes in the MOC are likely to include changes in marine ecosystem productivity, fisheries, ocean $\mathrm{CO}_{2}$ uptake, oceanic oxygen concentrations and terrestrial vegetation. Changes in terrestrial and ocean $\mathrm{CO}_{2}$ uptake may feed back on the climate system. $\{3.4\}$

\section{Adaptation and mitigation options ${ }^{14}$}

A wide array of adaptation options is available, but more extensive adaptation than is currently occurring is required to reduce vulnerability to climate change. There are barriers, limits and costs, which are not fully understood. $\{4.2\}$

Societies have a long record of managing the impacts of weather- and climate-related events. Nevertheless, additional adaptation measures will be required to reduce the adverse impacts of projected climate change and variability, regardless of the scale of mitigation undertaken over the next two to three decades. Moreover, vulnerability to climate change can be exacerbated by other stresses. These arise from, for example, current climate hazards, poverty and unequal access to resources, food insecurity, trends in economic globalisation, conflict and incidence of diseases such as HIV/AIDS. \{4.2\}

Some planned adaptation to climate change is already occurring on a limited basis. Adaptation can reduce vulner- ability, especially when it is embedded within broader sectoral initiatives (Table SPM.4). There is high confidence that there are viable adaptation options that can be implemented in some sectors at low cost, and/or with high benefit-cost ratios. However, comprehensive estimates of global costs and benefits of adaptation are limited. $\{4.2$, Table 4.1$\}$

Adaptive capacity is intimately connected to social and economic development but is unevenly distributed across and within societies. $\{4.2\}$

A range of barriers limits both the implementation and effectiveness of adaptation measures. The capacity to adapt is dynamic and is influenced by a society's productive base, including natural and man-made capital assets, social networks and entitlements, human capital and institutions, governance, national income, health and technology. Even societies with high adaptive capacity remain vulnerable to climate change, variability and extremes. $\{4.2\}$

Both bottom-up and top-down studies indicate that there is high agreement and much evidence of substantial economic potential for the mitigation of global GHG emissions over the coming decades that could offset the projected growth of global emissions or reduce emissions below current levels (Figures SPM.9, SPM.10). ${ }^{15}$ While top-down and bottom-up studies are in line at the global level (Figure SPM.9) there are considerable differences at the sectoral level. $\{4.3\}$

No single technology can provide all of the mitigation potential in any sector. The economic mitigation potential, which is generally greater than the market mitigation potential, can only be achieved when adequate policies are in place and barriers removed (Table SPM.5). \{4.3\}

Bottom-up studies suggest that mitigation opportunities with net negative costs have the potential to reduce emissions by around $6 \mathrm{GtCO}_{2}$-eq/yr in 2030, realising which requires dealing with implementation barriers. $\{4.3\}$

\footnotetext{
${ }^{14}$ While this Section deals with adaptation and mitigation separately, these responses can be complementary. This theme is discussed in Section 5 .

${ }^{15}$ The concept of 'mitigation potential' has been developed to assess the scale of GHG reductions that could be made, relative to emission baselines, for a given level of carbon price (expressed in cost per unit of carbon dioxide equivalent emissions avoided or reduced). Mitigation potential is further differentiated in terms of 'market mitigation potential' and 'economic mitigation potential'.

Market mitigation potential is the mitigation potential based on private costs and private discount rates (reflecting the perspective of private consumers and companies), which might be expected to occur under forecast market conditions, including policies and measures currently in place, noting that barriers limit actual uptake.

Economic mitigation potential is the mitigation potential that takes into account social costs and benefits and social discount rates (reflecting the perspective of society; social discount rates are lower than those used by private investors), assuming that market efficiency is improved by policies and measures and barriers are removed.

Mitigation potential is estimated using different types of approaches. Bottom-up studies are based on assessment of mitigation options, emphasising specific technologies and regulations. They are typically sectoral studies taking the macro-economy as unchanged. Top-down studies assess the economy-wide potential of mitigation options. They use globally consistent frameworks and aggregated information about mitigation options and capture macro-economic and market feedbacks.
} 
Table SPM.4. Selected examples of planned adaptation by sector. \{Table 4.1\}

\begin{tabular}{|c|c|c|c|}
\hline Sector & Adaptation option/strategy & Underlying policy framework & $\begin{array}{l}\text { Key constraints and opportunities } \\
\text { to implementation (Normal font = } \\
\text { constraints; italics = opportunities) }\end{array}$ \\
\hline Water & $\begin{array}{l}\text { Expanded rainwater harvesting; } \\
\text { water storage and conservation } \\
\text { techniques; water re-use; } \\
\text { desalination; water-use and } \\
\text { irrigation efficiency }\end{array}$ & $\begin{array}{l}\text { National water policies and } \\
\text { integrated water resources manage- } \\
\text { ment; water-related hazards } \\
\text { management }\end{array}$ & $\begin{array}{l}\text { Financial, human resources and } \\
\text { physical barriers; integrated water } \\
\text { resources management; synergies with } \\
\text { other sectors }\end{array}$ \\
\hline Agriculture & $\begin{array}{l}\text { Adjustment of planting dates and } \\
\text { crop variety; crop relocation; } \\
\text { improved land management, e.g. } \\
\text { erosion control and soil protection } \\
\text { through tree planting }\end{array}$ & $\begin{array}{l}\text { R\&D policies; institutional reform; } \\
\text { land tenure and land reform; training; } \\
\text { capacity building; crop insurance; } \\
\text { financial incentives, e.g. subsidies } \\
\text { and tax credits }\end{array}$ & $\begin{array}{l}\text { Technological and financial } \\
\text { constraints; access to new varieties; } \\
\text { markets; longer growing season in } \\
\text { higher latitudes; revenues from 'new' } \\
\text { products }\end{array}$ \\
\hline $\begin{array}{l}\text { Infrastructure/ } \\
\text { settlement } \\
\text { (including } \\
\text { coastal zones) }\end{array}$ & $\begin{array}{l}\text { Relocation; seawalls and storm } \\
\text { surge barriers; dune reinforce- } \\
\text { ment; land acquisition and } \\
\text { creation of marshlands/wetlands } \\
\text { as buffer against sea level rise } \\
\text { and flooding; protection of existing } \\
\text { natural barriers }\end{array}$ & $\begin{array}{l}\text { Standards and regulations that } \\
\text { integrate climate change consider- } \\
\text { ations into design; land-use policies; } \\
\text { building codes; insurance }\end{array}$ & $\begin{array}{l}\text { Financial and technological barriers; } \\
\text { availability of relocation space; } \\
\text { integrated policies and management; } \\
\text { synergies with sustainable development } \\
\text { goals }\end{array}$ \\
\hline Human health & $\begin{array}{l}\text { Heat-health action plans; } \\
\text { emergency medical services; } \\
\text { improved climate-sensitive } \\
\text { disease surveillance and control; } \\
\text { safe water and improved } \\
\text { sanitation }\end{array}$ & $\begin{array}{l}\text { Public health policies that recognise } \\
\text { climate risk; strengthened health } \\
\text { services; regional and international } \\
\text { cooperation }\end{array}$ & $\begin{array}{l}\text { Limits to human tolerance (vulnerable } \\
\text { groups); knowledge limitations; financial } \\
\text { capacity; upgraded health services; } \\
\text { improved quality of life }\end{array}$ \\
\hline Tourism & $\begin{array}{l}\text { Diversification of tourism } \\
\text { attractions and revenues; shifting } \\
\text { ski slopes to higher altitudes and } \\
\text { glaciers; artificial snow-making }\end{array}$ & $\begin{array}{l}\text { Integrated planning (e.g. carrying } \\
\text { capacity; linkages with other } \\
\text { sectors); financial incentives, e.g. } \\
\text { subsidies and tax credits }\end{array}$ & $\begin{array}{l}\text { Appeal/marketing of new attractions; } \\
\text { financial and logistical challenges; } \\
\text { potential adverse impact on other } \\
\text { sectors (e.g. artificial snow-making may } \\
\text { increase energy use); revenues from } \\
\text { 'new' attractions; involvement of wider } \\
\text { group of stakeholders }\end{array}$ \\
\hline Transport & $\begin{array}{l}\text { Ralignment/relocation; design } \\
\text { standards and planning for roads, } \\
\text { rail and other infrastructure to } \\
\text { cope with warming and drainage }\end{array}$ & $\begin{array}{l}\text { Integrating climate change consider- } \\
\text { ations into national transport policy; } \\
\text { investment in R\&D for special } \\
\text { situations, e.g. permafrost areas }\end{array}$ & $\begin{array}{l}\text { Financial and technological barriers; } \\
\text { availability of less vulnerable routes; } \\
\text { improved technologies and integration } \\
\text { with key sectors (e.g. energy) }\end{array}$ \\
\hline Energy & $\begin{array}{l}\text { Strengthening of overhead } \\
\text { transmission and distribution } \\
\text { infrastructure; underground } \\
\text { cabling for utilities; energy } \\
\text { efficiency; use of renewable } \\
\text { sources; reduced dependence on } \\
\text { single sources of energy }\end{array}$ & $\begin{array}{l}\text { National energy policies, regulations, } \\
\text { and fiscal and financial incentives to } \\
\text { encourage use of alternative } \\
\text { sources; incorporating climate } \\
\text { change in design standards }\end{array}$ & $\begin{array}{l}\text { Access to viable alternatives; financial } \\
\text { and technological barriers; acceptance } \\
\text { of new technologies; stimulation of new } \\
\text { technologies; use of local resources }\end{array}$ \\
\hline
\end{tabular}

Note:

Other examples from many sectors would include early warning systems.

Future energy infrastructure investment decisions, expected to exceed US\$20 trillion $^{16}$ between 2005 and 2030, will have long-term impacts on GHG emissions, because of the long lifetimes of energy plants and other infrastructure capital stock. The widespread diffusion of low-carbon technologies may take many decades, even if early investments in these technologies are made attractive. Initial estimates show that returning global energy-related $\mathrm{CO}_{2}$ emissions to 2005 levels by 2030 would require a large shift in investment patterns, although the net additional investment required ranges from negligible to 5 to $10 \%$. $\{4.3\}$

\footnotetext{
${ }^{16} 20$ trillion $=20,000$ billion $=20 \times 10^{12}$
} 


\section{Comparison between global economic mitigation potential and projected emissions increase in 2030}

a)

Gt $\mathrm{CO}_{2}$-eq

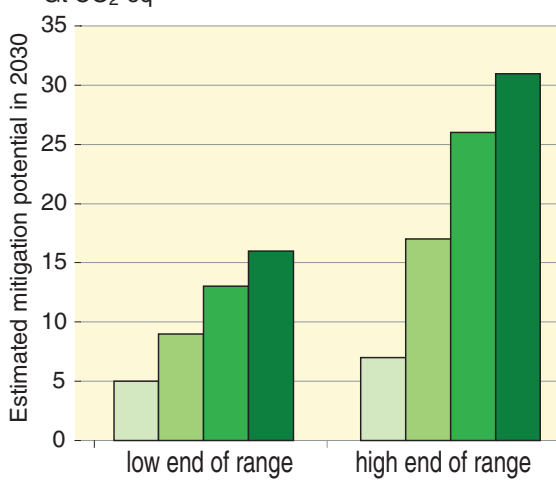

b) Top-down

$\square<20 \quad \square<50 \quad \square<100$ US\$ $/ \mathrm{tCO}_{2}$-eq

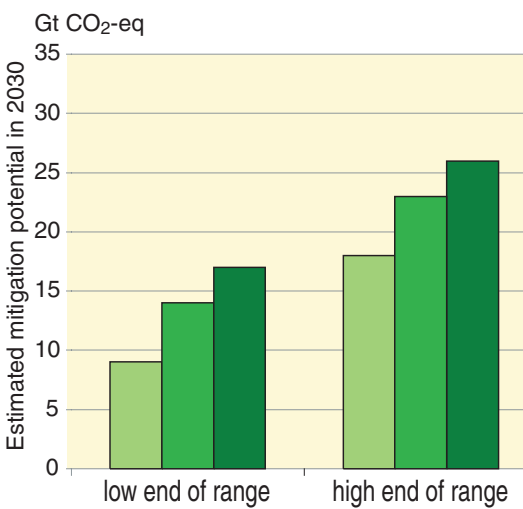

c) Increase in GHG emissions above year 2000 levels

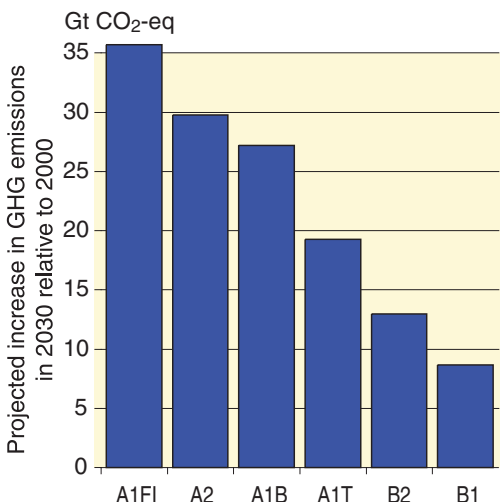

Figure SPM.9. Global economic mitigation potential in 2030 estimated from bottom-up (Panel a) and top-down (Panel b) studies, compared with the projected emissions increases from SRES scenarios relative to year $2000 \mathrm{GHG}$ emissions of $40.8 \mathrm{GtCO}_{2}$-eq (Panel c). Note: GHG emissions in 2000 are exclusive of emissions of decay of above ground biomass that remains after logging and deforestation and from peat fires and drained peat soils, to ensure consistency with the SRES emission results. \{Figure 4.1\}

Economic mitigation potentials by sector in 2030 estimated from bottom-up studies

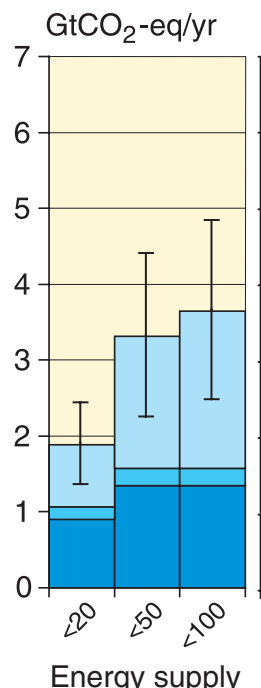

Energy supply

2.4-4.7

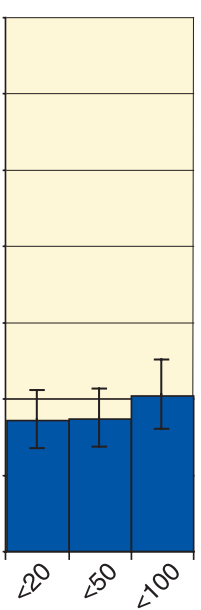

Transport

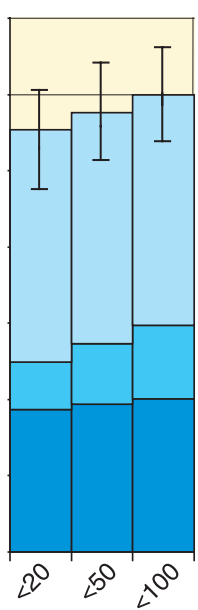

Buildings

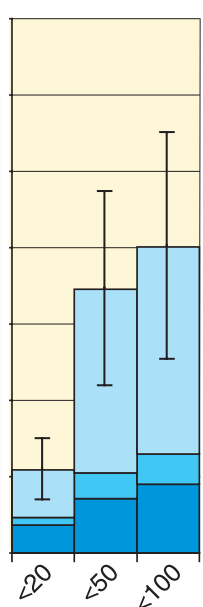

Industry

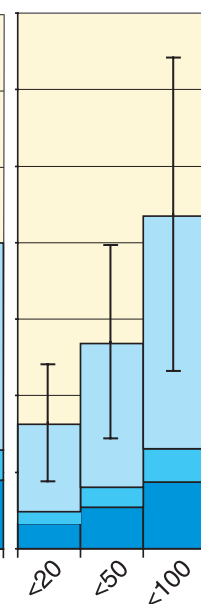

Agriculture

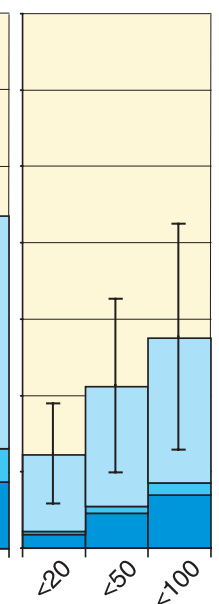

Forestry

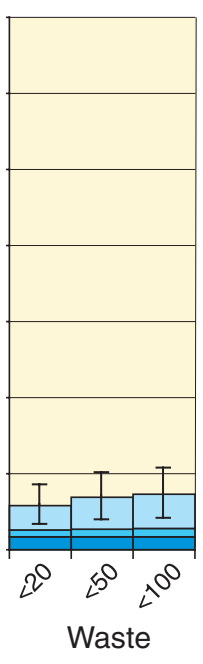

$\square$ Non-OECD/EIT

$\square$ EIT

$\square$ OECD

$\square$ World total

US $\$ \mathrm{tCO}_{2}$-eq

total sectoral potential at $<\mathrm{US} \$ 100 / \mathrm{tCO}_{2}$-eq in $\mathrm{GtCO}_{2}$-eq/yr:
$1.6-2.5$
5.3-6.7
2.5-5.5
2.3-6.4
$1.3-4.2$
0.4-1.0

Figure SPM.10. Estimated economic mitigation potential by sector in 2030 from bottom-up studies, compared to the respective baselines assumed in the sector assessments. The potentials do not include non-technical options such as lifestyle changes. \{Figure 4.2\}

Notes:

a) The ranges for global economic potentials as assessed in each sector are shown by vertical lines. The ranges are based on end-use allocations of emissions, meaning that emissions of electricity use are counted towards the end-use sectors and not to the energy supply sector.

b) The estimated potentials have been constrained by the availability of studies particularly at high carbon price levels.

c) Sectors used different baselines. For industry, the SRES B2 baseline was taken, for energy supply and transport, the World Energy Outlook (WEO) 2004 baseline was used; the building sector is based on a baseline in between SRES B2 and A1B; for waste, SRES A1B driving forces were used to construct a waste-specific baseline; agriculture and forestry used baselines that mostly used B2 driving forces.

d) Only global totals for transport are shown because international aviation is included.

e) Categories excluded are: non- $\mathrm{CO}_{2}$ emissions in buildings and transport, part of material efficiency options, heat production and co-generation in energy supply, heavy duty vehicles, shipping and high-occupancy passenger transport, most high-cost options for buildings, wastewater treatment, emission reduction from coal mines and gas pipelines, and fluorinated gases from energy supply and transport. The underestimation of the total economic potential from these emissions is of the order of 10 to $15 \%$. 


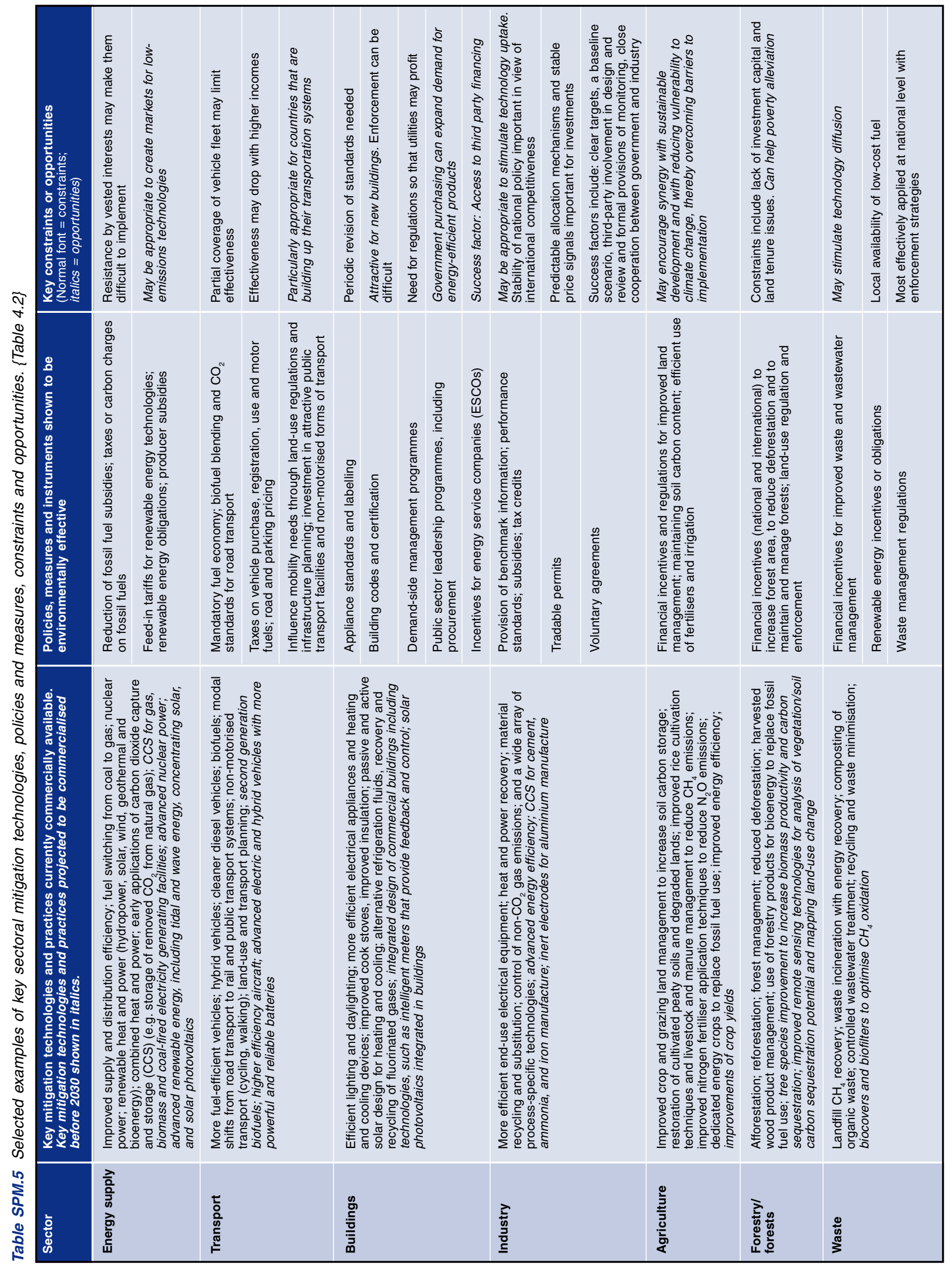


A wide variety of policies and instruments are available to governments to create the incentives for mitigation action. Their applicability depends on national circumstances and sectoral context (Table SPM.5). \{4.3\}

They include integrating climate policies in wider development policies, regulations and standards, taxes and charges, tradable permits, financial incentives, voluntary agreements, information instruments, and research, development and demonstration (RD\&D). $\{4.3\}$

An effective carbon-price signal could realise significant mitigation potential in all sectors. Modelling studies show that global carbon prices rising to US $\$ 20-80 / \mathrm{tCO}_{2}$-eq by 2030 are consistent with stabilisation at around 550ppm $\mathrm{CO}_{2}$-eq by 2100 . For the same stabilisation level, induced technological change may lower these price ranges to US\$5-65/tCO $\mathrm{CO}_{2}$-eq in 2030. ${ }^{17}\{4.3\}$

There is high agreement and much evidence that mitigation actions can result in near-term co-benefits (e.g. improved health due to reduced air pollution) that may offset a substantial fraction of mitigation costs. $\{4.3\}$

There is high agreement and medium evidence that Annex I countries' actions may affect the global economy and global emissions, although the scale of carbon leakage remains uncertain. $^{18}\{4.3\}$

Fossil fuel exporting nations (in both Annex I and non-Annex I countries) may expect, as indicated in the TAR, lower demand and prices and lower GDP growth due to mitigation policies. The extent of this spillover depends strongly on assumptions related to policy decisions and oil market conditions. $\{4.3\}$

There is also high agreement and medium evidence that changes in lifestyle, behaviour patterns and management practices can contribute to climate change mitigation across all sectors. $\{4.3\}$

Many options for reducing global GHG emissions through international cooperation exist. There is high agreement and much evidence that notable achievements of the UNFCCC and its Kyoto Protocol are the establishment of a global response to climate change, stimulation of an array of national policies, and the creation of an international carbon market and new institutional mechanisms that may provide the foundation for future mitigation efforts. Progress has also been made in addressing adaptation within the UNFCCC and additional international initiatives have been suggested. $\{4.5\}$

Greater cooperative efforts and expansion of market mechanisms will help to reduce global costs for achieving a given level of mitigation, or will improve environmental effectiveness. Efforts can include diverse elements such as emissions targets; sectoral, local, sub-national and regional actions; RD\&D programmes; adopting common policies; implementing development-oriented actions; or expanding financing instruments. $\{4.5\}$

In several sectors, climate response options can be implemented to realise synergies and avoid conflicts with other dimensions of sustainable development. Decisions about macroeconomic and other non-climate policies can significantly affect emissions, adaptive capacity and vulnerability. $\{4.4,5.8\}$

Making development more sustainable can enhance mitigative and adaptive capacities, reduce emissions and reduce vulnerability, but there may be barriers to implementation. On the other hand, it is very likely that climate change can slow the pace of progress towards sustainable development. Over the next half-century, climate change could impede achievement of the Millennium Development Goals. \{5.8\}

\section{The long-term perspective}

Determining what constitutes "dangerous anthropogenic interference with the climate system" in relation to Article 2 of the UNFCCC involves value judgements. Science can support informed decisions on this issue, including by providing criteria for judging which vulnerabilities might be labelled 'key'. \{Box 'Key Vulnerabilities and Article 2 of the UNFCCC', Topic 5\}

Key vulnerabilities ${ }^{19}$ may be associated with many climate-sensitive systems, including food supply, infrastructure, health, water resources, coastal systems, ecosystems, global biogeochemical cycles, ice sheets and modes of oceanic and atmospheric circulation. \{Box 'Key Vulnerabilities and Article 2 of the UNFCCC', Topic 5\}

\footnotetext{
${ }^{17}$ Studies on mitigation portfolios and macro-economic costs assessed in this report are based on top-down modelling. Most models use a global least-cost approach to mitigation portfolios, with universal emissions trading, assuming transparent markets, no transaction cost, and thus perfect implementation of mitigation measures throughout the $21^{\text {st }}$ century. Costs are given for a specific point in time. Global modelled costs will increase if some regions, sectors (e.g. land use), options or gases are excluded. Global modelled costs will decrease with lower baselines, use of revenues from carbon taxes and auctioned permits, and if induced technological learning is included. These models do not consider climate benefits and generally also co-benefits of mitigation measures, or equity issues. Significant progress has been achieved in applying approaches based on induced technological change to stabilisation studies; however, conceptual issues remain. In the models that consider induced technological change, projected costs for a given stabilisation level are reduced; the reductions are greater at lower stabilisation level.

${ }^{18}$ Further details may be found in Topic 4 of this Synthesis Report.

${ }^{19}$ Key vulnerabilities can be identified based on a number of criteria in the literature, including magnitude, timing, persistence/reversibility, the potential for adaptation, distributional aspects, likelihood and 'importance' of the impacts.
} 
The five 'reasons for concern' identified in the TAR remain a viable framework to consider key vulnerabilities. These 'reasons' are assessed here to be stronger than in the TAR. Many risks are identified with higher confidence. Some risks are projected to be larger or to occur at lower increases in temperature. Understanding about the relationship between impacts (the basis for 'reasons for concern' in the TAR) and vulnerability (that includes the ability to adapt to impacts) has improved. $\{5.2\}$

This is due to more precise identification of the circumstances that make systems, sectors and regions especially vulnerable and growing evidence of the risks of very large impacts on multiple-century time scales. $\{5.2\}$

- Risks to unique and threatened systems. There is new and stronger evidence of observed impacts of climate change on unique and vulnerable systems (such as polar and high mountain communities and ecosystems), with increasing levels of adverse impacts as temperatures increase further. An increasing risk of species extinction and coral reef damage is projected with higher confidence than in the TAR as warming proceeds. There is medium confidence that approximately 20 to $30 \%$ of plant and animal species assessed so far are likely to be at increased risk of extinction if increases in global average temperature exceed 1.5 to $2.5^{\circ} \mathrm{C}$ over $1980-1999$ levels. Confidence has increased that a 1 to $2^{\circ} \mathrm{C}$ increase in global mean temperature above 1990 levels (about 1.5 to $2.5^{\circ} \mathrm{C}$ above preindustrial) poses significant risks to many unique and threatened systems including many biodiversity hotspots. Corals are vulnerable to thermal stress and have low adaptive capacity. Increases in sea surface temperature of about 1 to $3^{\circ} \mathrm{C}$ are projected to result in more frequent coral bleaching events and widespread mortality, unless there is thermal adaptation or acclimatisation by corals. Increasing vulnerability of indigenous communities in the Arctic and small island communities to warming is projected. $\{5.2\}$

- Risks of extreme weather events. Responses to some recent extreme events reveal higher levels of vulnerability than the TAR. There is now higher confidence in the projected increases in droughts, heat waves and floods, as well as their adverse impacts. $\{5.2\}$

- Distribution of impacts and vulnerabilities. There are sharp differences across regions and those in the weakest economic position are often the most vulnerable to climate change. There is increasing evidence of greater vulnerability of specific groups such as the poor and elderly not only in developing but also in developed countries. Moreover, there is increased evidence that low-latitude and less developed areas generally face greater risk, for example in dry areas and megadeltas. $\{5.2\}$
- Aggregate impacts. Compared to the TAR, initial net market-based benefits from climate change are projected to peak at a lower magnitude of warming, while damages would be higher for larger magnitudes of warming. The net costs of impacts of increased warming are projected to increase over time. $\{5.2\}$

- Risks of large-scale singularities. There is high confidence that global warming over many centuries would lead to a sea level rise contribution from thermal expansion alone that is projected to be much larger than observed over the $20^{\text {th }}$ century, with loss of coastal area and associated impacts. There is better understanding than in the TAR that the risk of additional contributions to sea level rise from both the Greenland and possibly Antarctic ice sheets may be larger than projected by ice sheet models and could occur on century time scales. This is because ice dynamical processes seen in recent observations but not fully included in ice sheet models assessed in the AR4 could increase the rate of ice loss. $\{5.2\}$

There is high confidence that neither adaptation nor mitigation alone can avoid all climate change impacts; however, they can complement each other and together can significantly reduce the risks of climate change. $\{5.3\}$

Adaptation is necessary in the short and longer term to address impacts resulting from the warming that would occur even for the lowest stabilisation scenarios assessed. There are barriers, limits and costs, but these are not fully understood. Unmitigated climate change would, in the long term, be likely to exceed the capacity of natural, managed and human systems to adapt. The time at which such limits could be reached will vary between sectors and regions. Early mitigation actions would avoid further locking in carbon intensive infrastructure and reduce climate change and associated adaptation needs. $\{5.2,5.3\}$

Many impacts can be reduced, delayed or avoided by mitigation. Mitigation efforts and investments over the next two to three decades will have a large impact on opportunities to achieve lower stabilisation levels. Delayed emission reductions significantly constrain the opportunities to achieve lower stabilisation levels and increase the risk of more severe climate change impacts. $\{5.3,5.4,5.7\}$

In order to stabilise the concentration of GHGs in the atmosphere, emissions would need to peak and decline thereafter. The lower the stabilisation level, the more quickly this peak and decline would need to occur. ${ }^{20}\{5.4\}$

Table SPM.6 and Figure SPM.11 summarise the required emission levels for different groups of stabilisation concentrations and the resulting equilibrium global warming and long-

${ }^{20}$ For the lowest mitigation scenario category assessed, emissions would need to peak by 2015, and for the highest, by 2090 (see Table SPM.6). Scenarios that use alternative emission pathways show substantial differences in the rate of global climate change. 
term sea level rise due to thermal expansion only. ${ }^{21}$ The timing and level of mitigation to reach a given temperature stabilisation level is earlier and more stringent if climate sensitivity is high than if it is low. $\{5.4,5.7\}$

Sea level rise under warming is inevitable. Thermal expansion would continue for many centuries after GHG concentrations have stabilised, for any of the stabilisation levels assessed, causing an eventual sea level rise much larger than projected for the $21^{\text {st }}$ century. The eventual contributions from Greenland ice sheet loss could be several metres, and larger than from thermal expansion, should warming in excess of 1.9 to $4.6^{\circ} \mathrm{C}$ above pre-industrial be sustained over many centuries. The long time scales of thermal expansion and ice sheet response to warming imply that stabilisation of GHG concentrations at or above present levels would not stabilise sea level for many centuries. $\{5.3,5.4\}$

There is high agreement and much evidence that all stabilisation levels assessed can be achieved by deployment of a portfolio of technologies that are either currently available or expected to be commercialised in coming decades, assuming appropriate and effective incentives are in place for their development, acquisition, deployment and diffusion and addressing related barriers. $\{5.5\}$

All assessed stabilisation scenarios indicate that 60 to $80 \%$ of the reductions would come from energy supply and use and industrial processes, with energy efficiency playing a key role in many scenarios. Including non- $\mathrm{CO}_{2}$ and $\mathrm{CO}_{2}$ land-use and forestry mitigation options provides greater flexibility and cost-effectiveness. Low stabilisation levels require early investments and substantially more rapid diffusion and commercialisation of advanced low-emissions technologies. \{5.5\}

Without substantial investment flows and effective technology transfer, it may be difficult to achieve emission reduction at a significant scale. Mobilising financing of incremental costs of low-carbon technologies is important. $\{5.5\}$

Table SPM.6. Characteristics of post-TAR stabilisation scenarios and resulting long-term equilibrium global average temperature and the sea level rise component from thermal expansion only. ${ }^{a}$ \{Table 5.1\}

\begin{tabular}{|c|c|c|c|c|c|c|c|}
\hline 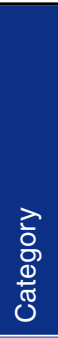 & 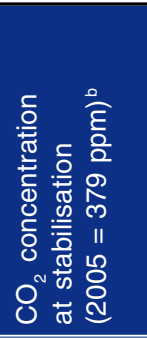 & 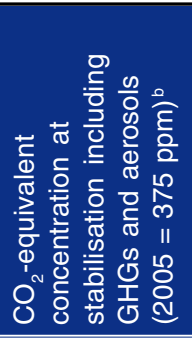 & 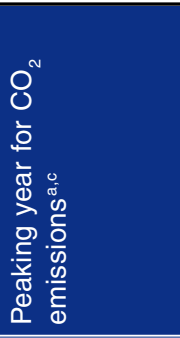 & 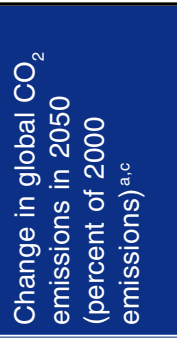 & 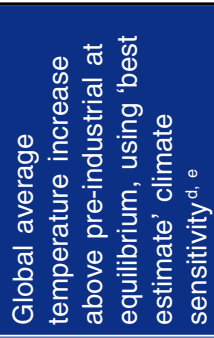 & 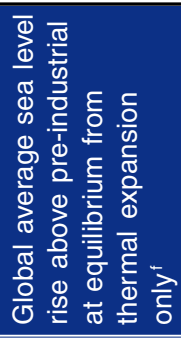 & 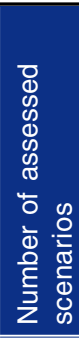 \\
\hline & ppm & ppm & year & percent & ${ }^{\circ} \mathrm{C}$ & metres & \\
\hline I & $350-400$ & $445-490$ & $2000-2015$ & -85 to -50 & $2.0-2.4$ & $0.4-1.4$ & 6 \\
\hline II & $400-440$ & $490-535$ & $2000-2020$ & -60 to -30 & $2.4-2.8$ & $0.5-1.7$ & 18 \\
\hline III & $440-485$ & $535-590$ & $2010-2030$ & -30 to +5 & $2.8-3.2$ & $0.6-1.9$ & 21 \\
\hline IV & $485-570$ & $590-710$ & $2020-2060$ & +10 to +60 & $3.2-4.0$ & $0.6-2.4$ & 118 \\
\hline V & $570-660$ & $710-855$ & $2050-2080$ & +25 to +85 & $4.0-4.9$ & $0.8-2.9$ & 9 \\
\hline VI & $660-790$ & $855-1130$ & $2060-2090$ & +90 to +140 & $4.9-6.1$ & $1.0-3.7$ & 5 \\
\hline
\end{tabular}

Notes:

a) The emission reductions to meet a particular stabilisation level reported in the mitigation studies assessed here might be underestimated due to missing carbon cycle feedbacks (see also Topic 2.3).

b) Atmospheric $\mathrm{CO}_{2}$ concentrations were $379 \mathrm{ppm}$ in 2005. The best estimate of total $\mathrm{CO}_{2}$-eq concentration in 2005 for all long-lived GHGs is about $455 \mathrm{ppm}$, while the corresponding value including the net effect of all anthropogenic forcing agents is $375 \mathrm{ppm} \mathrm{CO}_{2}$-eq.

c) Ranges correspond to the $15^{\text {th }}$ to $85^{\text {th }}$ percentile of the post-TAR scenario distribution. $\mathrm{CO}_{2}$ emissions are shown so multi-gas scenarios can be compared with $\mathrm{CO}_{2}$-only scenarios (see Figure SPM.3)

d) The best estimate of climate sensitivity is $3^{\circ} \mathrm{C}$

e) Note that global average temperature at equilibrium is different from expected global average temperature at the time of stabilisation of GHG concentrations due to the inertia of the climate system. For the majority of scenarios assessed, stabilisation of GHG concentrations occurs between 2100 and 2150 (see also Footnote 21).

f) Equilibrium sea level rise is for the contribution from ocean thermal expansion only and does not reach equilibrium for at least many centuries. These values have been estimated using relatively simple climate models (one low-resolution AOGCM and several EMICs based on the best estimate of $3^{\circ} \mathrm{C}$ climate sensitivity) and do not include contributions from melting ice sheets, glaciers and ice caps. Long-term thermal expansion is projected to result in 0.2 to $0.6 \mathrm{~m}$ per degree Celsius of global average warming above pre-industrial. (AOGCM refers to Atmosphere-Ocean General Circulation Model and EMICs to Earth System Models of Intermediate Complexity.)

\footnotetext{
${ }^{21}$ Estimates for the evolution of temperature over the course of this century are not available in the AR4 for the stabilisation scenarios. For most stabilisation levels, global average temperature is approaching the equilibrium level over a few centuries. For the much lower stabilisation scenarios (category I and II, Figure SPM.11), the equilibrium temperature may be reached earlier.
} 


\section{$\mathrm{CO}_{2}$ emissions and equilibrium temperature increases for a range of stabilisation levels}
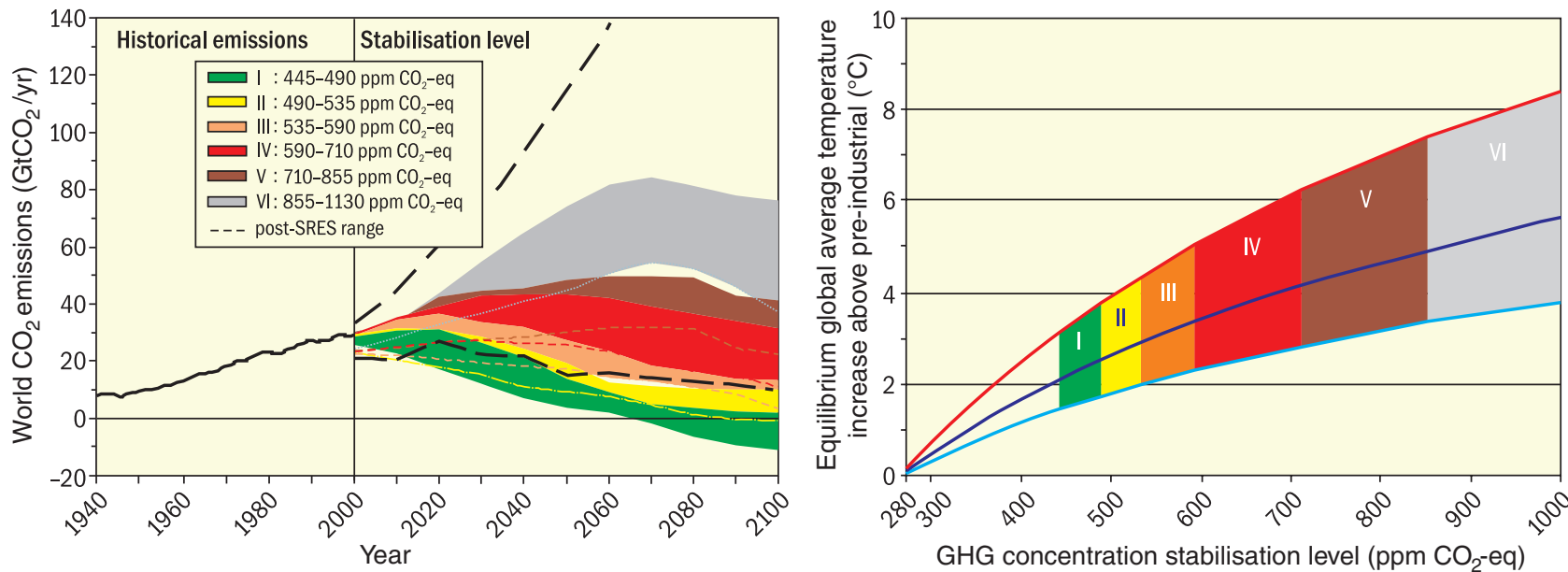

Figure SPM.11. Global $\mathrm{CO}_{2}$ emissions for 1940 to 2000 and emissions ranges for categories of stabilisation scenarios from 2000 to 2100 (lefthand panel); and the corresponding relationship between the stabilisation target and the likely equilibrium global average temperature increase above pre-industrial (right-hand panel). Approaching equilibrium can take several centuries, especially for scenarios with higher levels of stabilisation. Coloured shadings show stabilisation scenarios grouped according to different targets (stabilisation category I to VI). The right-hand panel shows ranges of global average temperature change above pre-industrial, using (i) 'best estimate' climate sensitivity of $3^{\circ} \mathrm{C}$ (black line in middle of shaded area), (ii) upper bound of likely range of climate sensitivity of $4.5^{\circ} \mathrm{C}$ (red line at top of shaded area) (iii) lower bound of likely range of climate sensitivity of $2^{\circ} \mathrm{C}$ (blue line at bottom of shaded area). Black dashed lines in the left panel give the emissions range of recent baseline scenarios published since the SRES (2000). Emissions ranges of the stabilisation scenarios comprise CO -only and multigas scenarios and correspond to the $10^{\text {th }}$ to $90^{\text {th }}$ percentile of the full scenario distribution. Note: $\mathrm{CO}_{2}$ emissions in most models do not include emissions from decay of above ground biomass that remains after logging and deforestation, and from peat fires and drained peat soils. \{Figure 5.1\}

The macro-economic costs of mitigation generally rise with the stringency of the stabilisation target (Table SPM.7). For specific countries and sectors, costs vary considerably from the global average. ${ }^{22}\{5.6\}$
In 2050, global average macro-economic costs for mitiga-

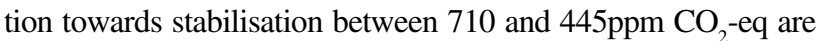
between a $1 \%$ gain and $5.5 \%$ decrease of global GDP (Table SPM.7). This corresponds to slowing average annual global GDP growth by less than 0.12 percentage points. $\{5.6\}$

Table SPM.7. Estimated global macro-economic costs in 2030 and 2050. Costs are relative to the baseline for least-cost trajectories towards different long-term stabilisation levels. \{Table 5.2\}

\begin{tabular}{|c|c|c|c|c|c|c|}
\hline \multirow[t]{2}{*}{$\begin{array}{l}\text { Stabilisation levels } \\
\left.\text { (ppm } \mathrm{CO}_{2}-\mathrm{eq}\right)\end{array}$} & \multicolumn{2}{|c|}{ Median GDP reduction ${ }^{a}(\%)$} & \multicolumn{2}{|c|}{ Range of GDP reduction ${ }^{\mathrm{b}}(\%)$} & \multicolumn{2}{|c|}{$\begin{array}{l}\text { Reduction of average annual GDP } \\
\text { growth rates (percentage points) }\end{array}$} \\
\hline & 2030 & 2050 & 2030 & 2050 & 2030 & 2050 \\
\hline $\begin{array}{l}445-535^{d} \\
535-590 \\
590-710\end{array}$ & $\begin{array}{l}0.6 \\
0.2\end{array}$ & $\begin{array}{l}\text { ailable } \\
1.3 \\
0.5\end{array}$ & $\begin{array}{l}<3 \\
0.2 \text { to } 2.5 \\
-0.6 \text { to } 1.2\end{array}$ & $\begin{array}{l}<5.5 \\
\text { slightly negative to } 4 \\
-1 \text { to } 2\end{array}$ & $\begin{array}{l}<0.12 \\
<0.1 \\
<0.06\end{array}$ & $\begin{array}{l}<0.12 \\
<0.1 \\
<0.05\end{array}$ \\
\hline
\end{tabular}

\section{Notes:}

Values given in this table correspond to the full literature across all baselines and mitigation scenarios that provide GDP numbers.

a) Global GDP based on market exchange rates.

b) The $10^{\text {th }}$ and $90^{\text {th }}$ percentile range of the analysed data are given where applicable. Negative values indicate GDP gain. The first row (445-535ppm $\mathrm{CO}_{2}$-eq) gives the upper bound estimate of the literature only.

c) The calculation of the reduction of the annual growth rate is based on the average reduction during the assessed period that would result in the indicated GDP decrease by 2030 and 2050 respectively.

d) The number of studies is relatively small and they generally use low baselines. High emissions baselines generally lead to higher costs.

e) The values correspond to the highest estimate for GDP reduction shown in column three.

\footnotetext{
${ }^{22}$ See Footnote 17 for more detail on cost estimates and model assumptions.
} 
Responding to climate change involves an iterative risk management process that includes both adaptation and mitigation and takes into account climate change damages, co-benefits, sustainability, equity and attitudes to risk. $\{5.1\}$

Impacts of climate change are very likely to impose net annual costs, which will increase over time as global temperatures increase. Peer-reviewed estimates of the social cost of carbon ${ }^{23}$ in 2005 average US\$12 per tonne of $\mathrm{CO}_{2}$, but the range from 100 estimates is large ( $-\$ 3$ to $\left.\$ 95 / \mathrm{tCO}_{2}\right)$. This is due in large part to differences in assumptions regarding climate sensitivity, response lags, the treatment of risk and equity, economic and non-economic impacts, the inclusion of potentially catastrophic losses and discount rates. Aggregate estimates of costs mask significant differences in impacts across sectors, regions and populations and very likely underestimate damage costs because they cannot include many nonquantifiable impacts. \{5.7\}

Limited and early analytical results from integrated analyses of the costs and benefits of mitigation indicate that they are broadly comparable in magnitude, but do not as yet permit an unambiguous determination of an emissions pathway or stabilisation level where benefits exceed costs. \{5.7\}

Climate sensitivity is a key uncertainty for mitigation scenarios for specific temperature levels. $\{5.4\}$

Choices about the scale and timing of GHG mitigation involve balancing the economic costs of more rapid emission reductions now against the corresponding medium-term and long-term climate risks of delay. \{5.7\}

${ }^{23} \mathrm{Net}$ economic costs of damages from climate change aggregated across the globe and discounted to the specified year. 


\section{Climate Change 2007: Synthesis Report}

\section{Synthesis Report}

\section{An Assessment of the Intergovernmental Panel on Climate Change}

This underlying report, adopted section by section at IPCC Plenary XXVII (Valencia, Spain, 12-17 November 2007), represents the formally agreed statement of the IPCC concerning key findings and uncertainties contained in the Working Group contributions to the Fourth Assessment Report.

Based on a draft prepared by:

\section{Core Writing Team}

Lenny Bernstein, Peter Bosch, Osvaldo Canziani, Zhenlin Chen, Renate Christ, Ogunlade Davidson, William Hare, Saleemul Huq, David Karoly, Vladimir Kattsov, Zbigniew Kundzewicz, Jian Liu, Ulrike Lohmann, Martin Manning, Taroh Matsuno, Bettina Menne, Bert Metz, Monirul Mirza, Neville Nicholls, Leonard Nurse, Rajendra Pachauri, Jean Palutikof, Martin Parry, Dahe Qin, Nijavalli Ravindranath, Andy Reisinger, Jiawen Ren, Keywan Riahi, Cynthia Rosenzweig, Matilde Rusticucci, Stephen Schneider, Youba Sokona, Susan Solomon, Peter Stott, Ronald Stouffer, Taishi Sugiyama, Rob Swart, Dennis Tirpak, Coleen Vogel, Gary Yohe

\section{Extended Writing Team}

Terry Barker

\section{Review Editors}

Abdelkader Allali, Roxana Bojariu, Sandra Diaz, Ismail Elgizouli, Dave Griggs, David Hawkins, Olav Hohmeyer, Bubu Pateh Jallow, Lučka Kajfež-Bogataj, Neil Leary, Hoesung Lee, David Wratt 

Introduction 


\section{Introduction}

This Synthesis Report is based on the assessment carried out by the three Working Groups (WGs) of the Intergovernmental Panel on Climate Change (IPCC). It provides an integrated view of climate change as the final part of the IPCC's Fourth Assessment Report (AR4).

Topic 1 summarises observed changes in climate and their effects on natural and human systems, regardless of their causes, while Topic 2 assesses the causes of the observed changes. Topic 3 presents projections of future climate change and related impacts under different scenarios.

Topic 4 discusses adaptation and mitigation options over the next few decades and their interactions with sustainable develop- ment. Topic 5 assesses the relationship between adaptation and mitigation on a more conceptual basis and takes a longer-term perspective. Topic 6 summarises the major robust findings and remaining key uncertainties in this assessment.

A schematic framework representing anthropogenic drivers, impacts of and responses to climate change, and their linkages, is shown in Figure I.1. At the time of the Third Assessment Report (TAR) in 2001, information was mainly available to describe the linkages clockwise, i.e. to derive climatic changes and impacts from socio-economic information and emissions. With increased understanding of these linkages, it is now possible to assess the linkages also counterclockwise, i.e. to evaluate possible development pathways and global emissions constraints that would reduce the risk of future impacts that society may wish to avoid.

\section{Schematic framework of anthropogenic climate change drivers, impacts and responses}

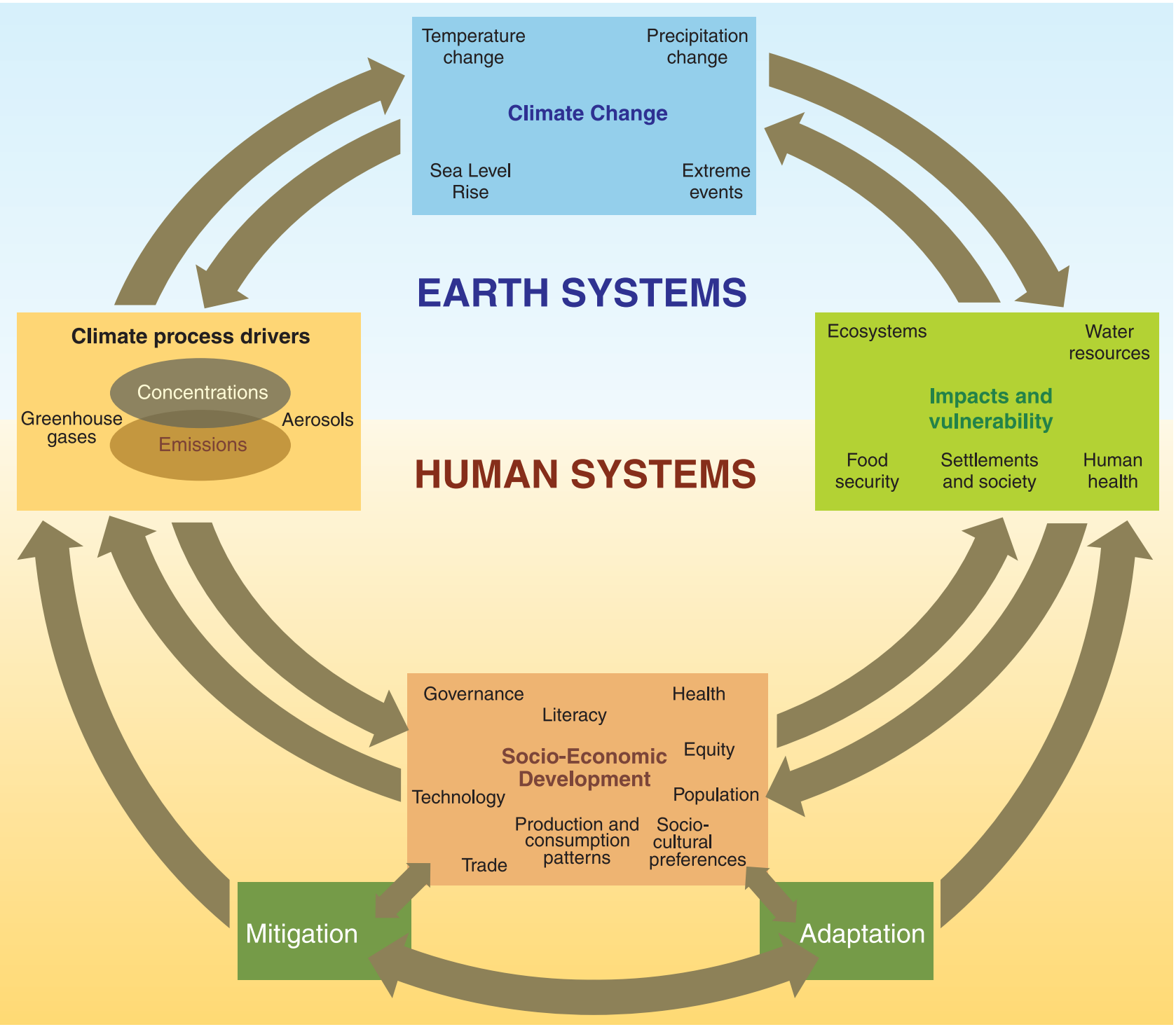

Figure I.1. Schematic framework representing anthropogenic drivers, impacts of and responses to climate change, and their linkages. 


\section{Treatment of uncertainty}

The IPCC uncertainty guidance note ${ }^{1}$ defines a framework for the treatment of uncertainties across all WGs and in this Synthesis Report. This framework is broad because the WGs assess material from different disciplines and cover a diversity of approaches to the treatment of uncertainty drawn from the literature. The nature of data, indicators and analyses used in the natural sciences is generally different from that used in assessing technology development or the social sciences. WG I focuses on the former, WG III on the latter, and WG II covers aspects of both.

Three different approaches are used to describe uncertainties each with a distinct form of language. Choices among and within these three approaches depend on both the nature of the information available and the authors' expert judgment of the correctness and completeness of current scientific understanding.

Where uncertainty is assessed qualitatively, it is characterised by providing a relative sense of the amount and quality of evidence (that is, information from theory, observations or models indicating whether a belief or proposition is true or valid) and the degree of agreement (that is, the level of concurrence in the literature on a particular finding). This approach is used by WG III through a series of self-explanatory terms such as: high agreement, much evidence; high agreement, medium evidence; medium agreement, medium evidence; etc.

Where uncertainty is assessed more quantitatively using expert judgement of the correctness of underlying data, models or analyses, then the following scale of confidence levels is used to express the assessed chance of a finding being correct: very high confidence at least 9 out of 10; high confidence about 8 out of 10; medium confidence about 5 out of 10; low confidence about 2 out of 10; and very low confidence less than 1 out of 10 .

Where uncertainty in specific outcomes is assessed using expert judgment and statistical analysis of a body of evidence (e.g. observations or model results), then the following likelihood ranges are used to express the assessed probability of occurrence: virtually certain $>99 \%$; extremely likely $>95 \%$; very likely $>90 \%$; likely $>66 \%$; more likely than not $>50 \%$; about as likely as not $33 \%$ to $66 \%$; unlikely $<33 \%$; very unlikely $<10 \%$; extremely unlikely $<5 \%$; exceptionally unlikely $<1 \%$.

WG II has used a combination of confidence and likelihood assessments and WG I has predominantly used likelihood assessments.

This Synthesis Report follows the uncertainty assessment of the underlying WGs. Where synthesised findings are based on information from more than one WG, the description of uncertainty used is consistent with that for the components drawn from the respective WG reports.

Unless otherwise stated, numerical ranges given in square brackets in this report indicate $90 \%$ uncertainty intervals (i.e. there is an estimated $5 \%$ likelihood that the value could be above the range given in square brackets and $5 \%$ likelihood that the value could be below that range). Uncertainty intervals are not necessarily symmetric around the best estimate.

\footnotetext{
${ }^{1}$ See http://www.ipcc.ch/meetings/ar4-workshops-express-meetings/uncertainty-guidance-note.pdf
} 

Observed changes in climate and their effects 


\subsection{Observations of climate change}

Since the TAR, progress in understanding how climate is changing in space and time has been gained through improvements and extensions of numerous datasets and data analyses, broader geographical coverage, better understanding of uncertainties and a wider variety of measurements. \{WGI SPM\}

\section{Definitions of climate change}

Climate change in IPCC usage refers to a change in the state of the climate that can be identified (e.g. using statistical tests) by changes in the mean and/or the variability of its properties, and that persists for an extended period, typically decades or longer. It refers to any change in climate over time, whether due to natural variability or as a result of human activity. This usage differs from that in the United Nations Framework Convention on Climate Change (UNFCCC), where climate change refers to a change of climate that is attributed directly or indirectly to human activity that alters the composition of the global atmosphere and that is in addition to natural climate variability observed over comparable time periods.

\section{Warming of the climate system is unequivocal, as is now evident from observations of increases in global average air and ocean temperatures, widespread melting of snow and ice and rising global average sea level (Figure 1.1). \{WGI 3.2, 4.8, 5.2, 5.5, SPM\}}

Eleven of the last twelve years (1995-2006) rank among the twelve warmest years in the instrumental record of global surface temperature (since 1850). The 100-year linear trend (1906-2005) of 0.74 [0.56 to 0.92$]^{\circ} \mathrm{C}$ is larger than the corresponding trend of 0.6 [0.4 to 0.8$]^{\circ} \mathrm{C}(1901-2000)$ given in the TAR (Figure 1.1). The linear warming trend over the 50 years from 1956 to $2005(0.13$ [0.10 to 0.16$]^{\circ} \mathrm{C}$ per decade) is nearly twice that for the 100 years from 1906 to 2005. \{WGI 3.2, SPM\}

The temperature increase is widespread over the globe and is greater at higher northern latitudes (Figure 1.2). Average Arctic temperatures have increased at almost twice the global average rate in the past 100 years. Land regions have warmed faster than the oceans (Figures 1.2 and 2.5). Observations since 1961 show that the average temperature of the global ocean has increased to depths of at least $3000 \mathrm{~m}$ and that the ocean has been taking up over $80 \%$ of the heat being added to the climate system. New analyses of balloonborne and satellite measurements of lower- and mid-tropospheric temperature show warming rates similar to those observed in surface temperature. $\{$ WGI 3.2, 3.4, 5.2, SPM \}

Increases in sea level are consistent with warming (Figure 1.1). Global average sea level rose at an average rate of 1.8 [1.3 to 2.3$] \mathrm{mm}$ per year over 1961 to 2003 and at an average rate of about 3.1 [2.4 to $3.8 \mathrm{Jmm}$ per year from 1993 to 2003 . Whether this faster rate for 1993 to 2003 reflects decadal variation or an increase in the longer- term trend is unclear. Since 1993 thermal expansion of the oceans has contributed about $57 \%$ of the sum of the estimated individual contributions to the sea level rise, with decreases in glaciers and ice caps contributing about $28 \%$ and losses from the polar ice sheets contributing the remainder. From 1993 to 2003 the sum of these climate contributions is consistent within uncertainties with the total sea level rise that is directly observed. (WGI 4.6, 4.8, 5.5, SPM, Table SPM.1\}

Observed decreases in snow and ice extent are also consistent with warming (Figure 1.1). Satellite data since 1978 show that annual average Arctic sea ice extent has shrunk by 2.7 [2.1 to 3.3$] \%$ per decade, with larger decreases in summer of 7.4 [5.0 to 9.8 ]\% per decade. Mountain glaciers and snow cover on average have declined in both hemispheres. The maximum areal extent of seasonally frozen ground has decreased by about $7 \%$ in the Northern Hemisphere since 1900, with decreases in spring of up to $15 \%$. Temperatures at the top of the permafrost layer have generally increased since the $1980 \mathrm{~s}$ in the Arctic by up to $3^{\circ} \mathrm{C}$. \{WGI 3.2, 4.5, 4.6, 4.7, 4.8, 5.5, SPM\}

At continental, regional and ocean basin scales, numerous longterm changes in other aspects of climate have also been observed. Trends from 1900 to 2005 have been observed in precipitation amount in many large regions. Over this period, precipitation increased significantly in eastern parts of North and South America, northern Europe and northern and central Asia whereas precipitation declined in the Sahel, the Mediterranean, southern Africa and parts of southern Asia. Globally, the area affected by drought has likely ${ }^{2}$ increased since the 1970s. [WGI 3.3, 3.9, SPM\}

Some extreme weather events have changed in frequency and/ or intensity over the last 50 years:

- It is very likely that cold days, cold nights and frosts have become less frequent over most land areas, while hot days and hot nights have become more frequent. \{WGI 3.8, SPM \}

- It is likely that heat waves have become more frequent over most land areas. \{WGI 3.8, SPM\}

- It is likely that the frequency of heavy precipitation events (or proportion of total rainfall from heavy falls) has increased over most areas. \{WGI 3.8, 3.9, SPM \}

- It is likely that the incidence of extreme high sea level ${ }^{3}$ has increased at a broad range of sites worldwide since 1975. \{WGI 5.5, SPM

There is observational evidence of an increase in intense tropical cyclone activity in the North Atlantic since about 1970, and suggestions of increased intense tropical cyclone activity in some other regions where concerns over data quality are greater. Multi-decadal variability and the quality of the tropical cyclone records prior to routine satellite observations in about 1970 complicate the detection of longterm trends in tropical cyclone activity. \{WGI 3.8, SPM\}

Average Northern Hemisphere temperatures during the second half of the $20^{\text {th }}$ century were very likely higher than during any other 50-year period in the last 500 years and likely the highest in at least the past 1300 years. $\{$ WGI 6.6, SPM \}

\footnotetext{
${ }^{2}$ Likelihood and confidence statements in italics represent calibrated expressions of uncertainty and confidence. See Box 'Treatment of uncertainty' in the Introduction for an explanation of these terms.

${ }^{3}$ Excluding tsunamis, which are not due to climate change. Extreme high sea level depends on average sea level and on regional weather systems. It is defined here as the highest $1 \%$ of hourly values of observed sea level at a station for a given reference period.
} 
Changes in temperature, sea level and Northern Hemisphere snow cover

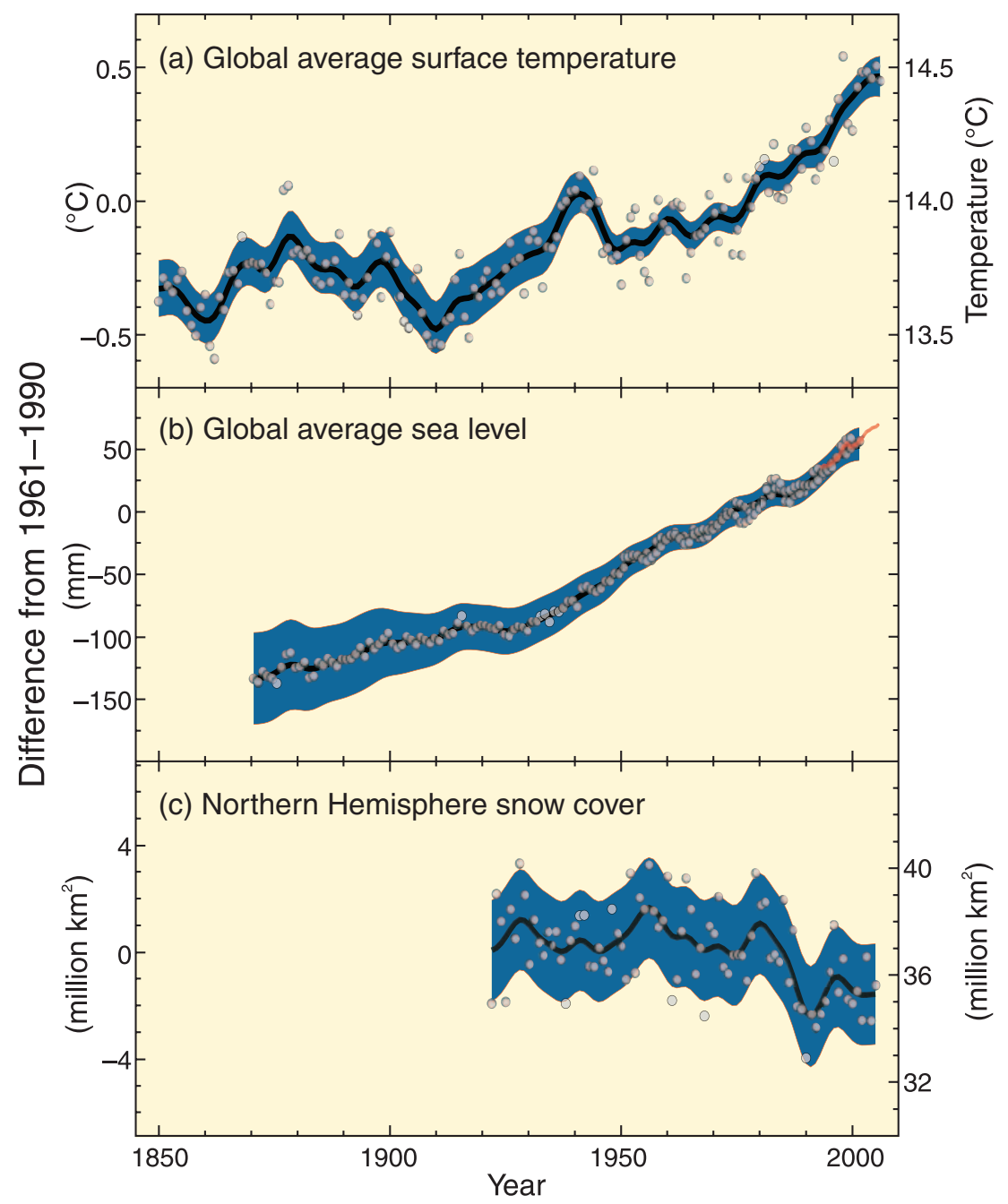

Figure 1.1. Observed changes in (a) global average surface temperature; (b) global average sea level from tide gauge (blue) and satellite (red) data; and (c) Northern Hemisphere snow cover for March-April. All differences are relative to corresponding averages for the period 1961-1990. Smoothed curves represent decadal averaged values while circles show yearly values. The shaded areas are the uncertainty intervals estimated from a comprehensive analysis of known uncertainties ( $a$ and $b$ ) and from the time series (c). \{WGI FAQ 3.1 Figure 1, Figure 4.2, Figure 5.13, Figure SPM.3\}

\subsection{Observed effects of climate changes}

The statements presented here are based largely on data sets that cover the period since 1970 . The number of studies of observed trends in the physical and biological environment and their relationship to regional climate changes has increased greatly since the TAR. The quality of the data sets has also improved. There is a notable lack of geographic balance in data and literature on observed changes, with marked scarcity in developing countries. \{WGII SPM\}

These studies have allowed a broader and more confident assessment of the relationship between observed warming and impacts than was made in the TAR. That assessment concluded that "there is high confidence ${ }^{2}$ that recent regional changes in temperature have had discernible impacts on physical and biological systems". \{WGII SPM\}
Observational evidence from all continents and most oceans shows that many natural systems are being affected by regional climate changes, particularly temperature increases. \{WGII SPM\}

There is high confidence that natural systems related to snow, ice and frozen ground (including permafrost) are affected. Examples are:

- enlargement and increased numbers of glacial lakes \{WGII 1.3, SPM\}

- increasing ground instability in permafrost regions and rock avalanches in mountain regions [WGII 1.3, SPM]

- changes in some Arctic and Antarctic ecosystems, including those in sea-ice biomes, and predators at high levels of the food web. \{WGII 1.3, 4.4, 15.4, SPM\}

Based on growing evidence, there is high confidence that the following effects on hydrological systems are occurring: increased runoff and earlier spring peak discharge in many glacier- and snowfed rivers, and warming of lakes and rivers in many regions, with effects on thermal structure and water quality. \{WGII 1.3, 15.2, SPM\} 
Changes in physical and biological systems and surface temperature 1970-2004

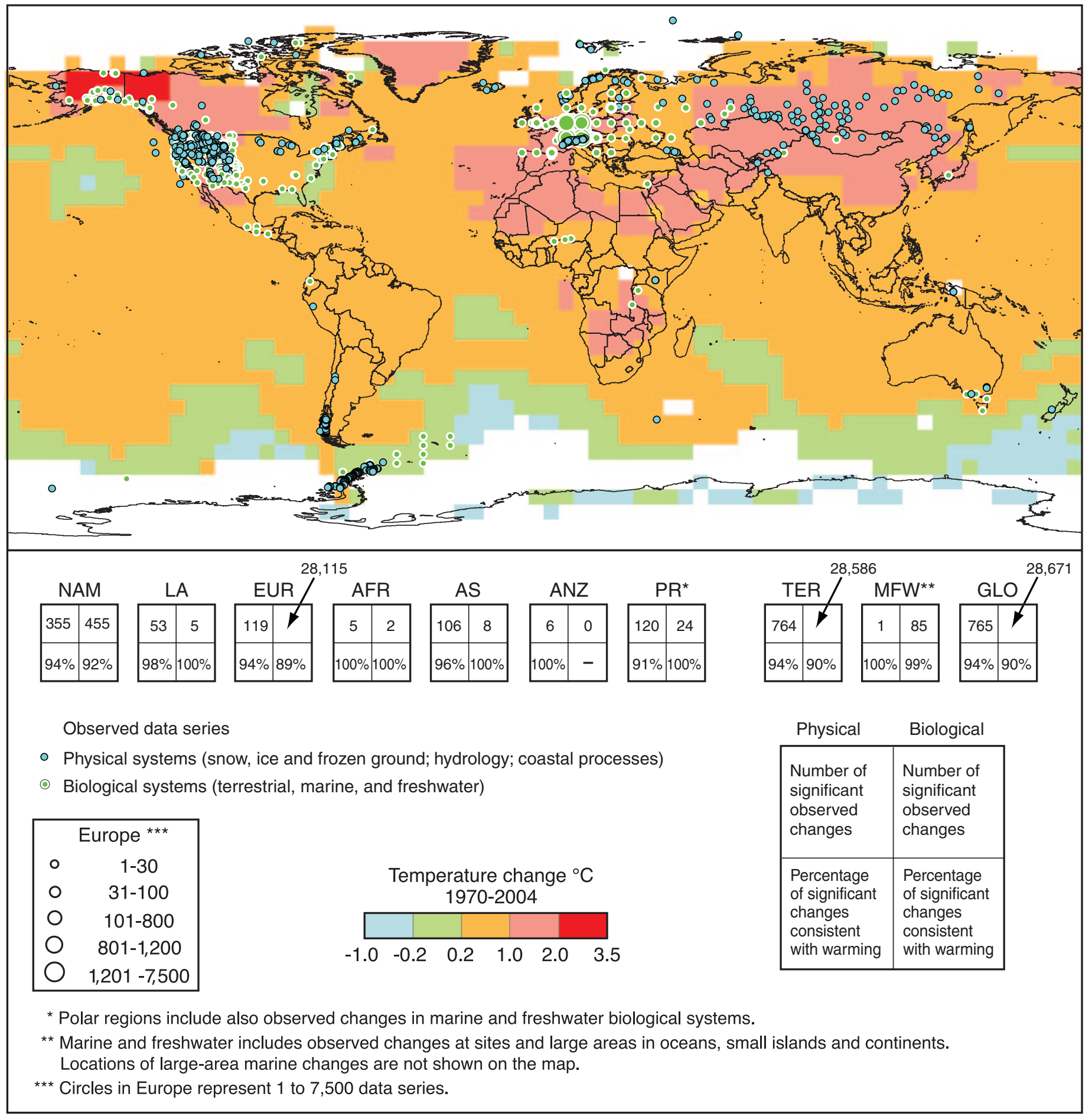

Figure 1.2. Locations of significant changes in data series of physical systems (snow, ice and frozen ground; hydrology; and coastal processes) and biological systems (terrestrial, marine, and freshwater biological systems), are shown together with surface air temperature changes over the period 19702004. A subset of about 29,000 data series was selected from about 80,000 data series from 577 studies. These met the following criteria: (1) ending in 1990 or later; (2) spanning a period of at least 20 years; and (3) showing a significant change in either direction, as assessed in individual studies. These data series are from about 75 studies (of which about 70 are new since the TAR) and contain about 29,000 data series, of which about 28,000 are from European studies. White areas do not contain sufficient observational climate data to estimate a temperature trend. The $2 \times 2$ boxes show the total number of data series with significant changes (top row) and the percentage of those consistent with warming (bottom row) for (i) continental regions: North America (NAM), Latin America (LA), Europe (EUR), Africa (AFR), Asia (AS), Australia and New Zealand (ANZ), and Polar Regions (PR) and (ii) global-scale: Terrestrial (TER), Marine and Freshwater (MFW), and Global (GLO). The numbers of studies from the seven regional boxes (NAM, ..., PR) do not add up to the global (GLO) totals because numbers from regions except Polar do not include the numbers related to Marine and Freshwater (MFW) systems. Locations of largearea marine changes are not shown on the map. \{WGIl Figure SPM.1, Figure 1.8, Figure 1.9; WGI Figure 3.9b\} 
There is very high confidence, based on more evidence from a wider range of species, that recent warming is strongly affecting terrestrial biological systems, including such changes as earlier timing of spring events, such as leaf-unfolding, bird migration and egg-laying; and poleward and upward shifts in ranges in plant and animal species. Based on satellite observations since the early 1980s, there is high confidence that there has been a trend in many regions towards earlier 'greening' of vegetation in the spring linked to longer thermal growing seasons due to recent warming. \{WGII 1.3, 8.2, 14.2, $S P M\}$

There is high confidence, based on substantial new evidence, that observed changes in marine and freshwater biological systems are associated with rising water temperatures, as well as related changes in ice cover, salinity, oxygen levels and circulation. These include: shifts in ranges and changes in algal, plankton and fish abundance in high-latitude oceans; increases in algal and zooplankton abundance in high-latitude and high-altitude lakes; and range changes and earlier fish migrations in rivers. While there is increasing evidence of climate change impacts on coral reefs, separating the impacts of climate-related stresses from other stresses (e.g. overfishing and pollution) is difficult. \{WGII 1.3, SPM \}

Other effects of regional climate changes on natural and human environments are emerging, although many are difficult to discern due to adaptation and non-climatic drivers. \{WGII SPM\}

Effects of temperature increases have been documented with medium confidence in the following managed and human systems:

- agricultural and forestry management at Northern Hemisphere higher latitudes, such as earlier spring planting of crops, and alterations in disturbances of forests due to fires and pests $\{$ WGII 1.3, $S P M\}$

- some aspects of human health, such as excess heat-related mortality in Europe, changes in infectious disease vectors in parts of Europe, and earlier onset of and increases in seasonal production of allergenic pollen in Northern Hemisphere high and mid-latitudes [WGII 1.3, 8.2, 8.ES, SPM]

- some human activities in the Arctic (e.g. hunting and shorter travel seasons over snow and ice) and in lower-elevation alpine areas (such as limitations in mountain sports). \{WGII 1.3, SPM \}

Sea level rise and human development are together contributing to losses of coastal wetlands and mangroves and increasing damage from coastal flooding in many areas. However, based on the published literature, the impacts have not yet become established trends. \{WGII 1.3, 1.ES, SPM\}

\subsection{Consistency of changes in physical and biological systems with warming}

Changes in the ocean and on land, including observed decreases in snow cover and Northern Hemisphere sea ice extent, thinner sea ice, shorter freezing seasons of lake and river ice, glacier melt, decreases in permafrost extent, increases in soil temperatures and borehole temperature profiles, and sea level rise, provide additional evidence that the world is warming. \{WGI 3.9\}

Of the more than 29,000 observational data series, from 75 studies, that show significant change in many physical and biological systems, more than $89 \%$ are consistent with the direction of change expected as a response to warming (Figure 1.2). \{WGII 1.4, SPM\}

\subsection{Some aspects of climate have not been observed to change}

Some aspects of climate appear not to have changed and, for some, data inadequacies mean that it cannot be determined if they have changed. Antarctic sea ice extent shows inter-annual variability and localised changes but no statistically significant average multi-decadal trend, consistent with the lack of rise in near-surface atmospheric temperatures averaged across the continent. There is insufficient evidence to determine whether trends exist in some other variables, for example the meridional overturning circulation (MOC) of the global ocean or small-scale phenomena such as tornadoes, hail, lightning and dust storms. There is no clear trend in the annual numbers of tropical cyclones. [WGI 3.2, 3.8, 4.4, 5.3, SPM) 

2

Causes of change 


\section{Causes of change}

This Topic considers both natural and anthropogenic drivers of climate change, including the chain from greenhouse gas (GHG) emissions to atmospheric concentrations to radiative forcing ${ }^{4}$ to climate responses and effects.

\subsection{Emissions of long-lived GHGs}

The radiative forcing of the climate system is dominated by the long-lived GHGs, and this section considers those whose emissions are covered by the UNFCCC.

\section{Global GHG emissions due to human activities have grown since pre-industrial times, with an increase of $70 \%$ between 1970 and 2004 (Figure 2.1). ${ }^{5}$ \{WGIII 1.3, SPM $\}$}

Carbon dioxide $\left(\mathrm{CO}_{2}\right)$ is the most important anthropogenic GHG. Its annual emissions have grown between 1970 and 2004 by about $80 \%$, from 21 to 38 gigatonnes (Gt), and represented $77 \%$ of total anthropogenic GHG emissions in 2004 (Figure 2.1). The rate of growth of $\mathrm{CO}_{2}$-eq emissions was much higher during the recent 10-year period of 1995-2004 (0.92 $\mathrm{GtCO}_{2}$-eq per year) than during the previous period of $1970-1994$ (0.43 $\mathrm{GtCO}_{2}$-eq per year). (WWIII 1.3, TS.1, SPM\}

\section{Carbon dioxide-equivalent $\left(\mathrm{CO}_{2}\right.$-eq) emissions and concentrations}

GHGs differ in their warming influence (radiative forcing) on the global climate system due to their different radiative properties and lifetimes in the atmosphere. These warming influences may be expressed through a common metric based on the radiative forcing of $\mathrm{CO}_{2}$.

- $\mathrm{CO}_{2}$-equivalent emission is the amount of $\mathrm{CO}_{2}$ emission that would cause the same time-integrated radiative forcing, over a given time horizon, as an emitted amount of a longlived GHG or a mixture of GHGs. The equivalent $\mathrm{CO}_{2}$ emission is obtained by multiplying the emission of a GHG by its Global Warming Potential (GWP) for the given time horizon. ${ }^{6}$ For a mix of GHGs it is obtained by summing the equivalent $\mathrm{CO}_{2}$ emissions of each gas. Equivalent $\mathrm{CO}_{2}$ emission is a standard and useful metric for comparing emissions of different GHGs but does not imply the same climate change responses (see WGI 2.10).

- $\mathrm{CO}_{2}$-equivalent concentration is the concentration of $\mathrm{CO}_{2}$ that would cause the same amount of radiative forcing as a given mixture of $\mathrm{CO}_{2}$ and other forcing components.

The largest growth in GHG emissions between 1970 and 2004 has come from energy supply, transport and industry, while residential and commercial buildings, forestry (including deforestation) and agriculture sectors have been growing at a lower rate. The

Global anthropogenic GHG emissions
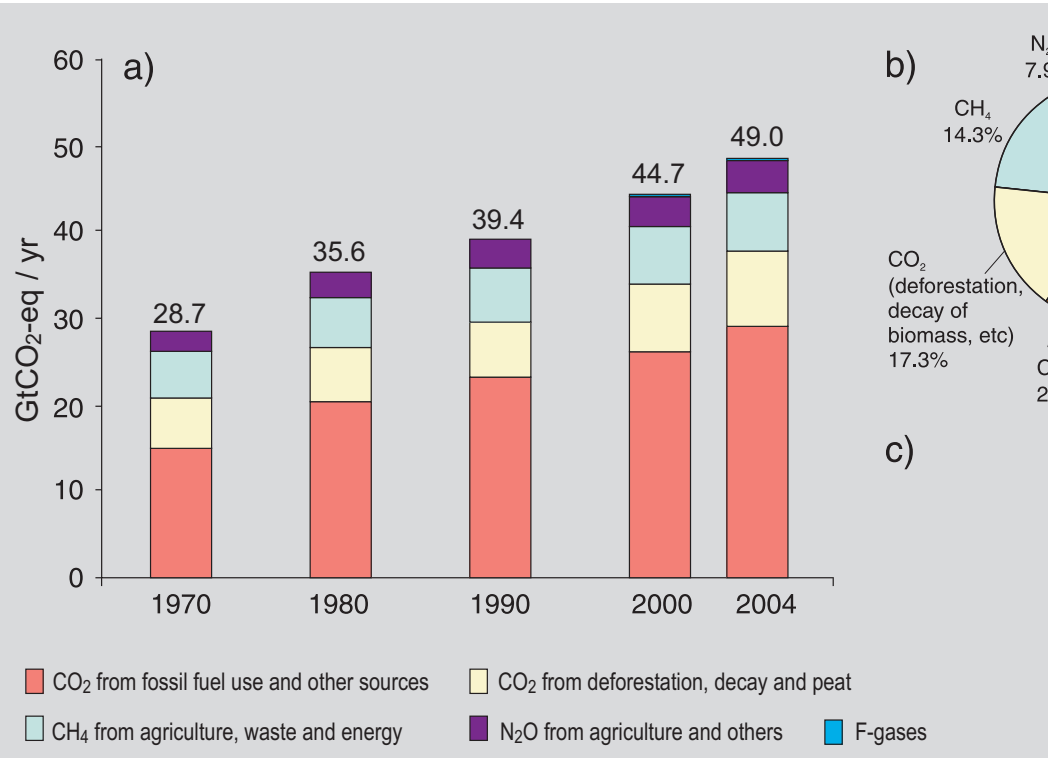

F-gases

c)

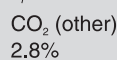

$2.8 \% \quad$ Waste and wastewater

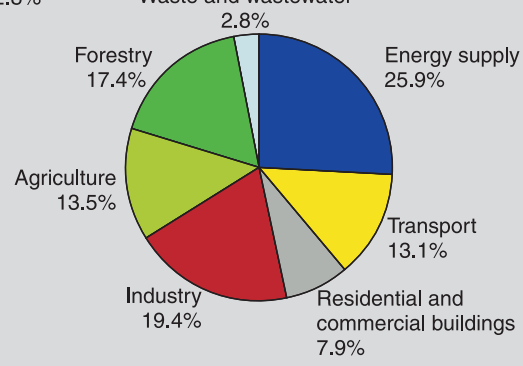

Figure 2.1. (a) Global annual emissions of anthropogenic GHGs from 1970 to $2004 .^{5}$ (b) Share of different anthropogenic GHGs in total emissions in 2004 in terms of $\mathrm{CO}_{2}$-eq. (c) Share of different sectors in total anthropogenic GHG emissions in 2004 in terms of $\mathrm{CO}_{2}$-eq. (Forestry includes deforestation.) \{WGIII Figures TS.1a, TS.1b, TS.2b\}

\footnotetext{
${ }^{4}$ Radiative forcing is a measure of the influence a factor has in altering the balance of incoming and outgoing energy in the Earth-atmosphere system and is an index of the importance of the factor as a potential climate change mechanism. In this report radiative forcing values are for changes relative to preindustrial conditions defined at 1750 and are expressed in watts per square metre $\left(\mathrm{W} / \mathrm{m}^{2}\right)$.

${ }^{5}$ Includes only carbon dioxide $\left(\mathrm{CO}_{2}\right)$, methane $\left(\mathrm{CH}_{4}\right)$, nitrous oxide $\left(\mathrm{N}_{2} \mathrm{O}\right)$, hydrofluorocarbons (HFCs), perfluorocarbons (PFCs) and sulphurhexafluoride $\left(\mathrm{SF}_{6}\right)$, whose emissions are covered by the UNFCCC. These GHGs are weighted by their 100-year Global Warming Potentials (GWPs), using values consistent with reporting under the UNFCCC.

${ }^{6}$ This report uses 100-year GWPs and numerical values consistent with reporting under the UNFCCC.

${ }^{7}$ Such values may consider only GHGs, or a combination of GHGs and aerosols.
} 
sectoral sources of GHGs in 2004 are considered in Figure 2.1c. \{WGIII 1.3, SPM \}

The effect on global emissions of the decrease in global energy intensity (-33\%) during 1970 to 2004 has been smaller than the combined effect of global income growth $(77 \%)$ and global population growth $(69 \%)$; both drivers of increasing energy-related $\mathrm{CO}_{2}$ emissions. The long-term trend of declining $\mathrm{CO}_{2}$ emissions per unit of energy supplied reversed after 2000. \{WGIII 1.3, Figure SPM.2, SPM\}

Differences in per capita income, per capita emissions and energy intensity among countries remain significant. In 2004, UNFCCC Annex I countries held a $20 \%$ share in world population, produced 57\% of the world's Gross Domestic Product based on Purchasing Power Parity $\left(\mathrm{GDP}_{\mathrm{PPP}}\right)$ and accounted for $46 \%$ of global GHG emissions (Figure 2.2). \{WGIII 1.3, SPM\}

\subsection{Drivers of climate change}

Changes in the atmospheric concentrations of GHGs and aerosols, land cover and solar radiation alter the energy balance of the climate system and are drivers of climate change. They affect the absorption, scattering and emission of radiation within the atmosphere and at the Earth's surface. The resulting positive or negative changes in energy balance due to these factors are expressed as radiative forcing ${ }^{4}$, which is used to compare warming or cooling influences on global climate. \{WGI TS.2\}

Human activities result in emissions of four long-lived GHGs: $\mathrm{CO}_{2}$, methane $\left(\mathrm{CH}_{4}\right)$, nitrous oxide $\left(\mathrm{N}_{2} \mathrm{O}\right)$ and halocarbons (a group of gases containing fluorine, chlorine or bromine). Atmospheric concentrations of GHGs increase when emissions are larger than removal processes.

Global atmospheric concentrations of $\mathrm{CO}_{2}, \mathrm{CH}_{4}$ and $\mathrm{N}_{2} \mathrm{O}$ have increased markedly as a result of human activities since $\mathbf{1 7 5 0}$ and now far exceed pre-industrial values determined from ice cores spanning many thousands of years
(Figure 2.3). The atmospheric concentrations of $\mathrm{CO}_{2}$ and $\mathrm{CH}_{4}$ in 2005 exceed by far the natural range over the last 650,000 years. Global increases in $\mathrm{CO}_{2}$ concentrations are due primarily to fossil fuel use, with land-use change providing another significant but smaller contribution. It is very likely that the observed increase in $\mathrm{CH}_{4}$ concentration is predominantly due to agriculture and fossil fuel use. The increase in $\mathrm{N}_{2} \mathrm{O}$ concentration is primarily due to agriculture. \{WGI 2.3, 7.3, SPM\}

The global atmospheric concentration of $\mathrm{CO}_{2}$ increased from a pre-industrial value of about $280 \mathrm{ppm}$ to $379 \mathrm{ppm}$ in 2005 . The annual $\mathrm{CO}_{2}$ concentration growth rate was larger during the last 10 years (1995-2005 average: 1.9ppm per year) than it has been since the beginning of continuous direct atmospheric measurements (1960-2005 average: $1.4 \mathrm{ppm}$ per year), although there is year-toyear variability in growth rates. \{WGI 2.3, 7.3, SPM; WGIII 1.3\}

The global atmospheric concentration of $\mathrm{CH}_{4}$ has increased from a pre-industrial value of about $715 \mathrm{ppb}$ to $1732 \mathrm{ppb}$ in the early $1990 \mathrm{~s}$, and was $1774 \mathrm{ppb}$ in 2005. Growth rates have declined since the early 1990s, consistent with total emissions (sum of anthropogenic and natural sources) being nearly constant during this period. (WGI $2.3,7.4, S P M\}$

The global atmospheric $\mathrm{N}_{2} \mathrm{O}$ concentration increased from a pre-industrial value of about $270 \mathrm{ppb}$ to $319 \mathrm{ppb}$ in 2005 . (WGI 2.3, 7.4, SPM\}

Many halocarbons (including hydrofluorocarbons) have increased from a near-zero pre-industrial background concentration, primarily due to human activities. \{WGI 2.3, SPM; SROC SPM\}

There is very high confidence that the global average net effect of human activities since $\mathbf{1 7 5 0}$ has been one of warming, with a radiative forcing of $+1.6[+0.6$ to +2.4$] \mathrm{W} / \mathrm{m}^{2}$ (Figure 2.4). \{WGI 2.3, 6.5, 2.9, SPM $\}$

The combined radiative forcing due to increases in $\mathrm{CO}_{2}, \mathrm{CH}_{4}$ and $\mathrm{N}_{2} \mathrm{O}$ is $+2.3[+2.1$ to +2.5$] \mathrm{W} / \mathrm{m}^{2}$, and its rate of increase during

Regional distribution of GHG emissions by population and by GDP ${ }_{\mathrm{PPP}}$

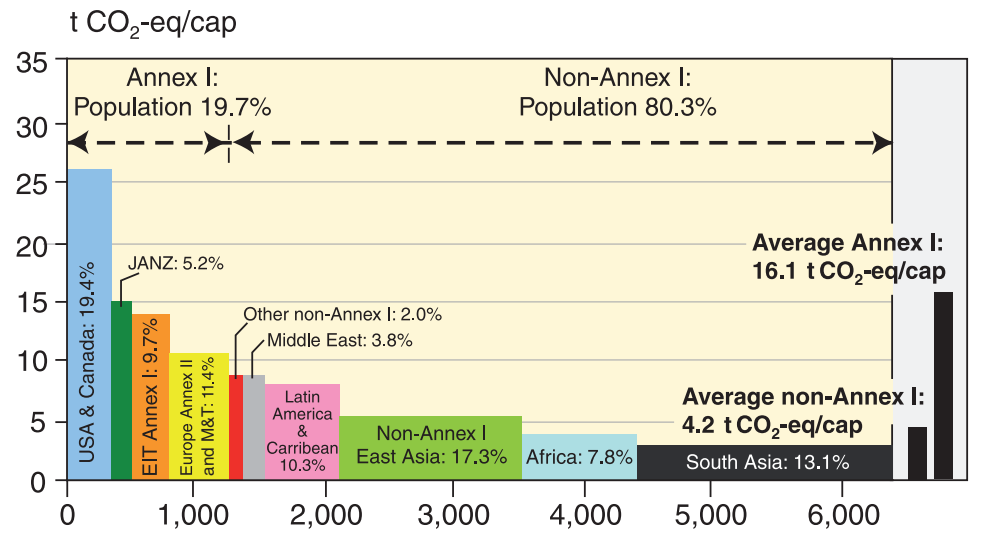

a)

Cumulative population in million

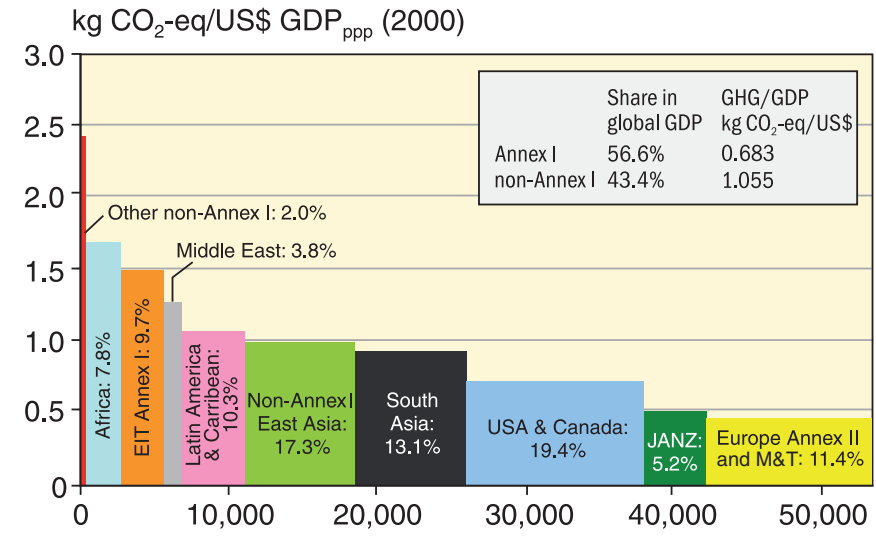

b) Cumulative $\mathrm{GDP}_{\mathrm{ppp}}(2000)$ in billion US\$

Figure 2.2. (a) Distribution of regional per capita GHG emissions according to the population of different country groupings in 2004 (see appendix for definitions of country groupings). (b) Distribution of regional GHG emissions per US\$ of GDP ${ }_{P P P}$ over the GDP of different country groupings in 2004. The percentages in the bars in both panels indicate a region's share in global GHG emissions. \{WGIII Figures SPM.3a, SPM.3b\} 
Changes in GHGs from ice core and modern data
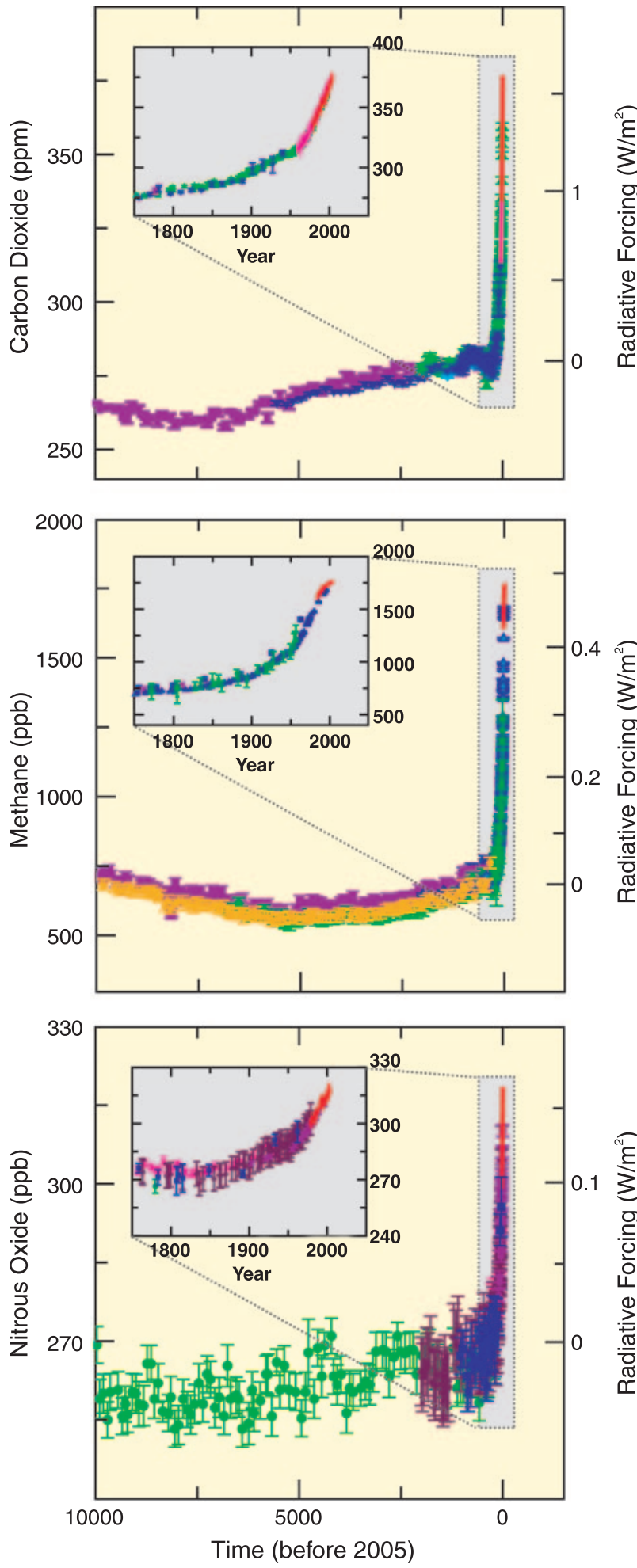

Figure 2.3. Atmospheric concentrations of $\mathrm{CO}_{2}, \mathrm{CH}_{4}$ and $\mathrm{N}_{2} \mathrm{O}$ over the last 10,000 years (large panels) and since 1750 (inset panels). Measurements are shown from ice cores (symbols with different colours for different studies) and atmospheric samples (red lines). The corresponding radiative forcings relative to 1750 are shown on the right hand axes of the large panels. \{WGI Figure SPM.1\} the industrial era is very likely to have been unprecedented in more than 10,000 years (Figures 2.3 and 2.4). The $\mathrm{CO}_{2}$ radiative forcing increased by $20 \%$ from 1995 to 2005 , the largest change for any decade in at least the last 200 years. \{WGI 2.3, 6.4, SPM \}

Anthropogenic contributions to aerosols (primarily sulphate, organic carbon, black carbon, nitrate and dust) together produce a cooling effect, with a total direct radiative forcing of -0.5 [-0.9 to $-0.1] \mathrm{W} / \mathrm{m}^{2}$ and an indirect cloud albedo forcing of $-0.7[-1.8$ to $-0.3] \mathrm{W} / \mathrm{m}^{2}$. Aerosols also influence precipitation. \{WGI 2.4, 2.9, 7.5, $S P M\}$

In comparison, changes in solar irradiance since 1750 are estimated to have caused a small radiative forcing of +0.12 [+0.06 to $+0.30] \mathrm{W} / \mathrm{m}^{2}$, which is less than half the estimate given in the TAR. \{WGI 2.7, SPM\}

\subsection{Climate sensitivity and feedbacks}

The equilibrium climate sensitivity is a measure of the climate system response to sustained radiative forcing. It is defined as the equilibrium global average surface warming following a doubling of $\mathrm{CO}_{2}$ concentration. Progress since the TAR enables an assessment that climate sensitivity is likely to be in the range of 2 to $4.5^{\circ} \mathrm{C}$ with a best estimate of about $3^{\circ} \mathrm{C}$, and is very unlikely to be less than $1.5^{\circ} \mathrm{C}$. Values substantially higher than $4.5^{\circ} \mathrm{C}$ cannot be excluded, but agreement of models with observations is not as good for those values. [WGI 8.6, 9.6, Box 10.2, SPM)

Feedbacks can amplify or dampen the response to a given forcing. Direct emission of water vapour (a greenhouse gas) by human activities makes a negligible contribution to radiative forcing. However, as global average temperature increases, tropospheric water vapour concentrations increase and this represents a key positive feedback but not a forcing of climate change. Water vapour changes represent the largest feedback affecting equilibrium climate sensitivity and are now better understood than in the TAR. Cloud feedbacks remain the largest source of uncertainty. Spatial patterns of climate response are largely controlled by climate processes and feedbacks. For example, sea-ice albedo feedbacks tend to enhance the high latitude response. \{WGI 2.8, 8.6, 9.2, TS.2.1.3, TS.2.5, SPM\}

Warming reduces terrestrial and ocean uptake of atmospheric $\mathrm{CO}_{2}$, increasing the fraction of anthropogenic emissions remaining in the atmosphere. This positive carbon cycle feedback leads to larger atmospheric $\mathrm{CO}_{2}$ increases and greater climate change for a given emissions scenario, but the strength of this feedback effect varies markedly among models. \{WGI 7.3, TS.5.4, SPM; WGII 4.4\}

\subsection{Attribution of climate change}

Attribution evaluates whether observed changes are quantitatively consistent with the expected response to external forcings (e.g. changes in solar irradiance or anthropogenic GHGs) and inconsistent with alternative physically plausible explanations. [WGI TS.4, SPM\} 
Radiative forcing components

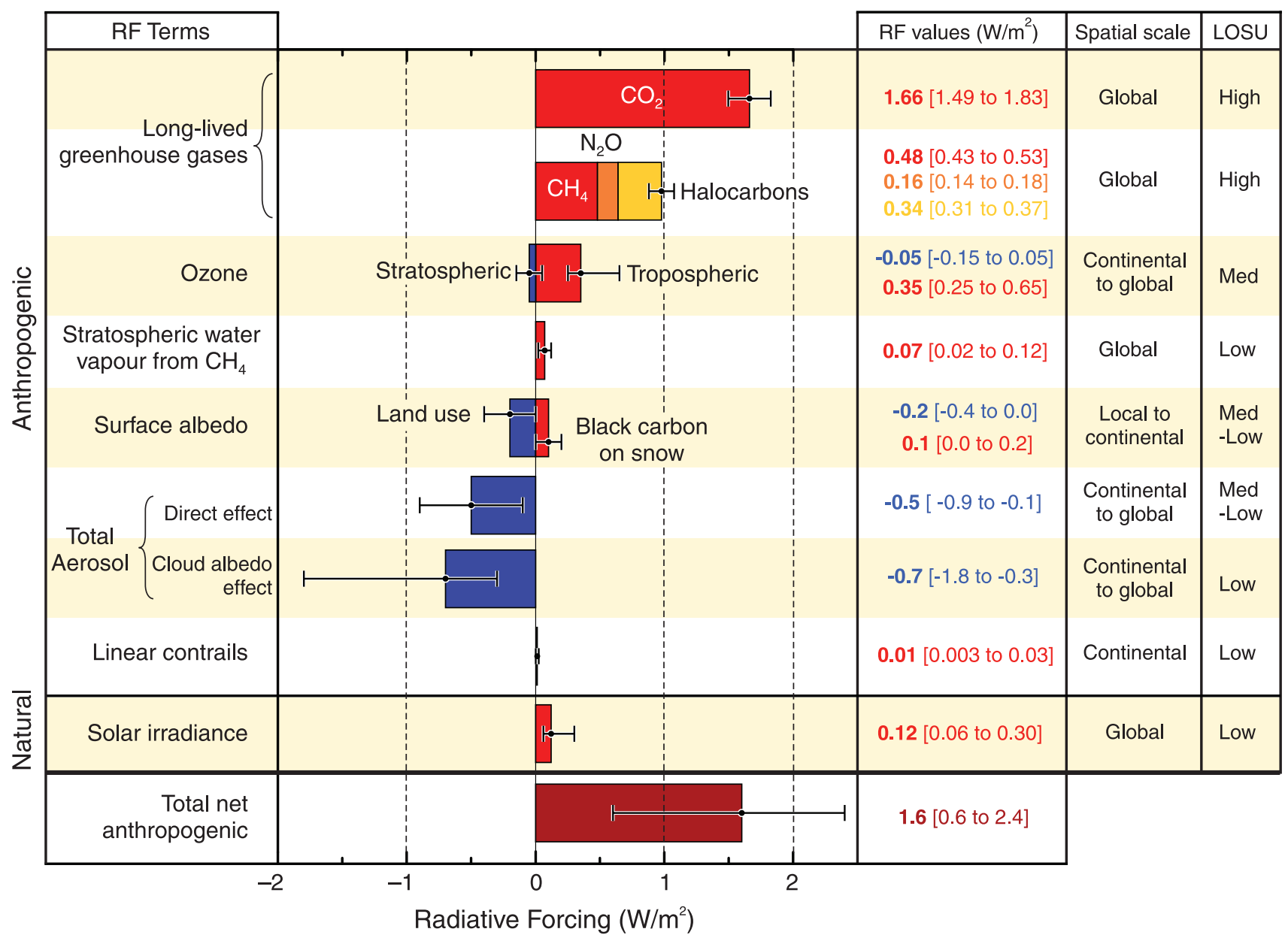

Figure 2.4. Global average radiative forcing (RF) in 2005 (best estimates and 5 to $95 \%$ uncertainty ranges) with respect to 1750 for $\mathrm{CO}_{2}, \mathrm{CH}_{4}, \mathrm{~N}_{2} \mathrm{O}$ and other important agents and mechanisms, together with the typical geographical extent (spatial scale) of the forcing and the assessed level of scientific understanding (LOSU). Aerosols from explosive volcanic eruptions contribute an additional episodic cooling term for a few years following an eruption. The range for linear contrails does not include other possible effects of aviation on cloudiness. \{WGI Figure SPM.2\}

Most of the observed increase in global average temperatures since the mid-20 ${ }^{\text {th }}$ century is very likely due to the observed increase in anthropogenic GHG concentrations. ${ }^{8}$ This is an advance since the TAR's conclusion that "most of the observed warming over the last $\mathbf{5 0}$ years is likely to have been due to the increase in GHG concentrations" (Figure 2.5). \{WGI 9.4, SPM\}

The observed widespread warming of the atmosphere and ocean, together with ice mass loss, support the conclusion that it is extremely unlikely that global climate change of the past 50 years can be explained without external forcing and very likely that it is not due to known natural causes alone. During this period, the sum of solar and volcanic forcings would likely have produced cooling, not warming. Warming of the climate system has been detected in changes in surface and atmospheric temperatures and in temperatures of the upper several hundred metres of the ocean. The observed pattern of tropospheric warming and stratospheric cooling is very likely due to the combined influences of GHG increases and stratospheric ozone depletion. It is likely that increases in GHG concentrations alone would have caused more warming than observed because volcanic and anthropogenic aerosols have offset some warming that would otherwise have taken place. \{WGI 2.9, 3.2, 3.4, 4.8, 5.2, 7.5, 9.4, 9.5, 9.7, TS.4.1, SPM \}

It is likely that there has been significant anthropogenic warming over the past $\mathbf{5 0}$ years averaged over each continent (except Antarctica) (Figure 2.5). \{WGI 3.2, 9.4, SPM $\}$

The observed patterns of warming, including greater warming over land than over the ocean, and their changes over time, are simulated only by models that include anthropogenic forcing. No coupled global climate model that has used natural forcing only has reproduced the continental mean warming trends in individual continents (except Antarctica) over the second half of the $20^{\text {th }}$ century. \{WGI 3.2, 9.4, TS.4.2, SPM \}

\footnotetext{
${ }^{8}$ Consideration of remaining uncertainty is based on current methodologies.
} 
Global and continental temperature change

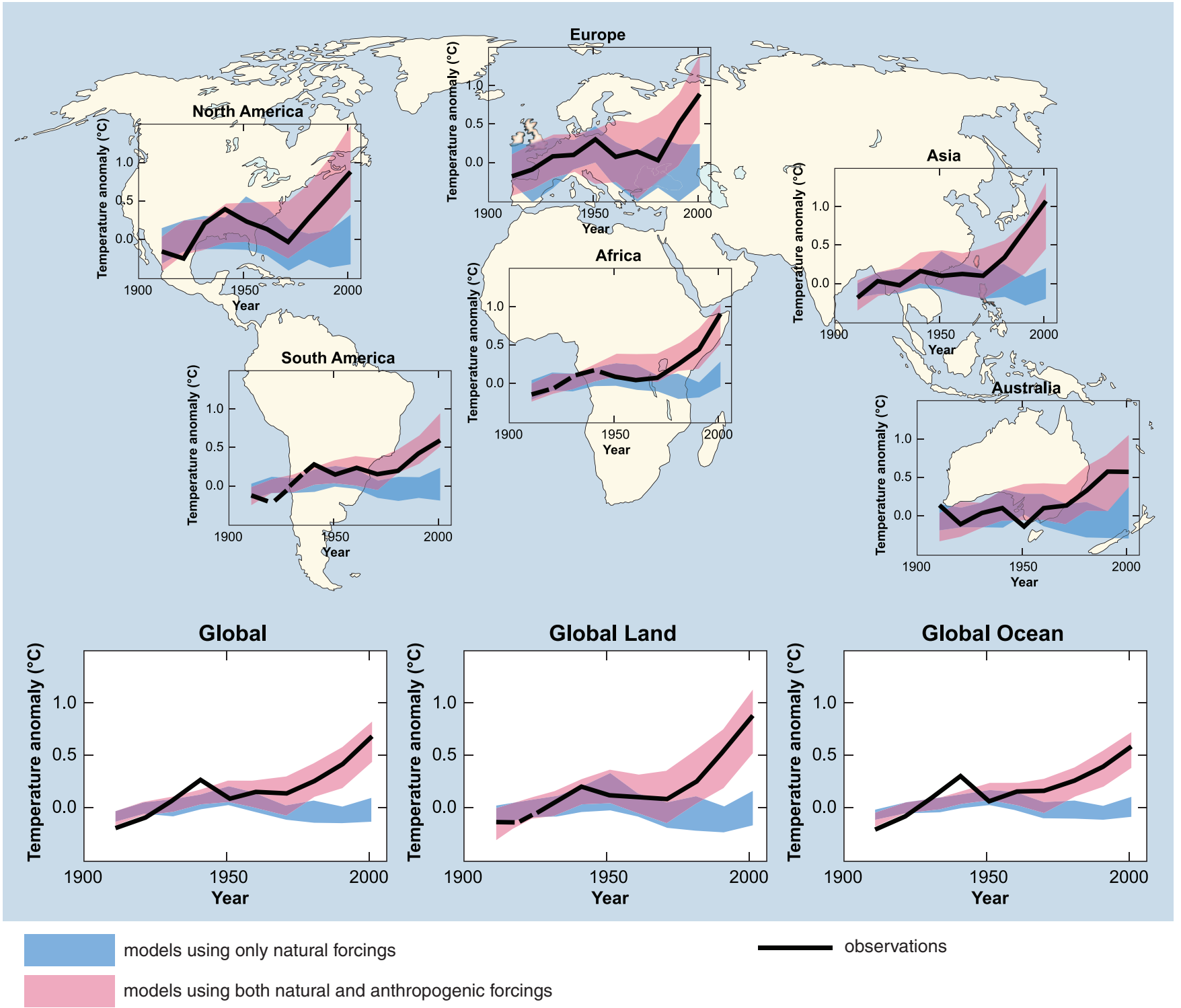

Figure 2.5. Comparison of observed continental- and global-scale changes in surface temperature with results simulated by climate models using either natural or both natural and anthropogenic forcings. Decadal averages of observations are shown for the period 1906-2005 (black line) plotted against the centre of the decade and relative to the corresponding average for the 1901-1950. Lines are dashed where spatial coverage is less than 50\%. Blue shaded bands show the 5 to $95 \%$ range for 19 simulations from five climate models using only the natural forcings due to solar activity and volcanoes. Red shaded bands show the 5 to 95\% range for 58 simulations from 14 climate models using both natural and anthropogenic forcings. \{WGI Figure SPM.4\}

Difficulties remain in simulating and attributing observed temperature changes at smaller scales. On these scales, natural climate variability is relatively larger, making it harder to distinguish changes expected due to external forcings. Uncertainties in local forcings, such as those due to aerosols and land-use change, and feedbacks also make it difficult to estimate the contribution of GHG increases to observed small-scale temperature changes. \{WGI 8.3, 9.4, SPM\}

Advances since the TAR show that discernible human influences extend beyond average temperature to other aspects of climate, including temperature extremes and wind patterns. \{WGI 9.4, 9.5, SPM $\}$
Temperatures of the most extreme hot nights, cold nights and cold days are likely to have increased due to anthropogenic forcing. It is more likely than not that anthropogenic forcing has increased the risk of heat waves. Anthropogenic forcing is likely to have contributed to changes in wind patterns, affecting extra-tropical storm tracks and temperature patterns in both hemispheres. However, the observed changes in the Northern Hemisphere circulation are larger than simulated by models in response to $20^{\text {th }}$ century forcing change. \{WGI 3.5, 3.6, 9.4, 9.5, 10.3, SPM\}

It is very likely that the response to anthropogenic forcing contributed to sea level rise during the latter half of the $20^{\text {th }}$ century. There is some evidence of the impact of human climatic influence 
on the hydrological cycle, including the observed large-scale patterns of changes in land precipitation over the $20^{\text {th }}$ century. It is more likely than not that human influence has contributed to a global trend towards increases in area affected by drought since the 1970 s and the frequency of heavy precipitation events. [WGI 3.3, 5.5, 9.5, TS.4.1, TS.4.3\}

Anthropogenic warming over the last three decades has likely had a discernible influence at the global scale on observed changes in many physical and biological systems. \{WGII 1.4\}

A synthesis of studies strongly demonstrates that the spatial agreement between regions of significant warming across the globe and the locations of significant observed changes in many natural systems consistent with warming is very unlikely to be due solely to natural variability of temperatures or natural variability of the systems. Several modelling studies have linked some specific responses in physical and biological systems to anthropogenic warming, but only a few such studies have been performed. Taken together with evidence of significant anthropogenic warming over the past 50 years averaged over each continent (except Antarctica), it is likely that anthropogenic warming over the last three decades has had a discernible influence on many natural systems. \{WGI 3.2, 9.4, SPM; WGII 1.4, SPM\}

Limitations and gaps currently prevent more complete attribution of the causes of observed natural system responses to anthropogenic warming. The available analyses are limited in the number of systems, length of records and locations considered. Natural temperature variability is larger at the regional than the global scale, thus affecting identification of changes to external forcing. At the regional scale, other non-climate factors (such as land-use change, pollution and invasive species) are influential. (WGII 1.2, 1.3, 1.4, SPM\} 

$\mathbf{3}$

Climate change and its impacts in the near and long term under different scenarios 


\subsection{Emissions scenarios}

There is high agreement and much evidence ${ }^{9}$ that with current climate change mitigation policies and related sustainable development practices, global GHG emissions will continue to grow over the next few decades. Baseline emissions scenarios published since the IPCC Special Report on Emissions Scenarios (SRES, 2000) are comparable in range to those presented in SRES (see Box on SRES scenarios and Figure 3.1)..$^{10}$ \{WGIII 1.3, 3.2, SPM\}

The SRES scenarios project an increase of baseline global GHG emissions by a range of 9.7 to $36.7 \mathrm{GtCO}_{2}$-eq ( 25 to $90 \%$ ) between 2000 and 2030. In these scenarios, fossil fuels are projected to maintain their dominant position in the global energy mix to 2030 and beyond. Hence $\mathrm{CO}_{2}$ emissions from energy use between 2000 and 2030 are projected to grow 40 to $110 \%$ over that period. \{WGIII 1.3, SPM

Studies published since SRES (i.e. post-SRES scenarios) have used lower values for some drivers for emissions, notably population projections. However, for those studies incorporating these new population projections, changes in other drivers, such as economic growth, result in little change in overall emission levels. Economic growth projections for Africa, Latin America and the Middle East to 2030 in post-SRES baseline scenarios are lower than in SRES, but this has only minor effects on global economic growth and overall emissions. \{WGIII 3.2, TS.3, SPM\}

Aerosols have a net cooling effect and the representation of aerosol and aerosol precursor emissions, including sulphur dioxide, black carbon and organic carbon, has improved in the postSRES scenarios. Generally, these emissions are projected to be lower than reported in SRES. \{WGIII 3.2, TS.3, SPM\}

Available studies indicate that the choice of exchange rate for Gross Domestic Product (GDP) (Market Exchange Rate, MER or
Scenarios for GHG emissions from 2000 to 2100 in the absence of additional climate policies

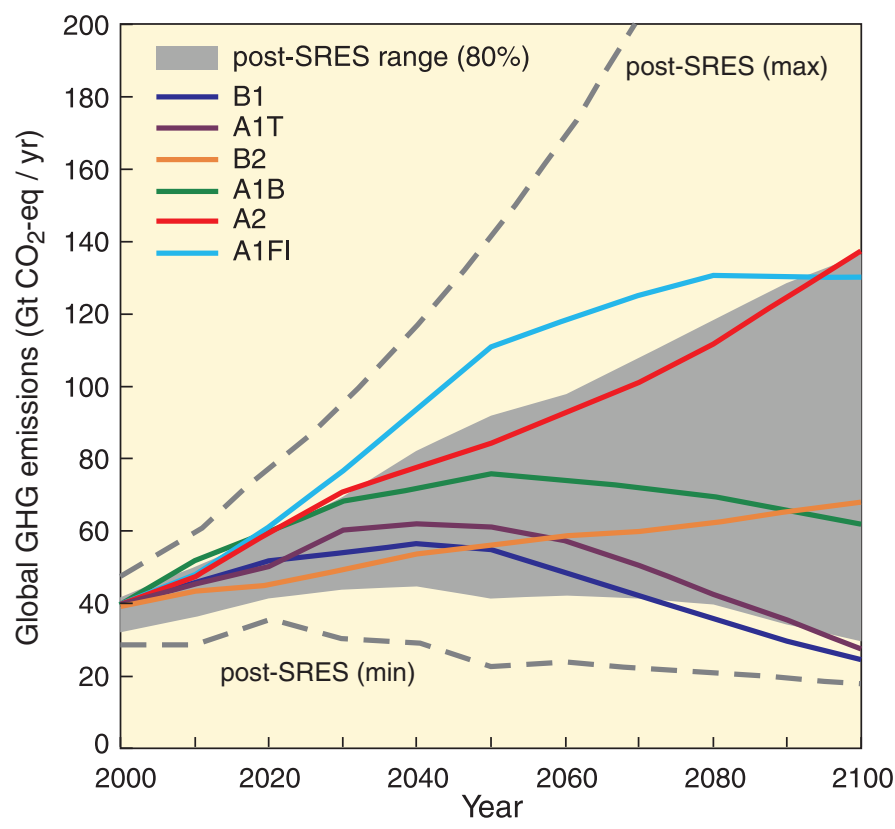

Figure 3.1. Global GHG emissions (in $\mathrm{GtCO}_{2}$-eq per year) in the absence of additional climate policies: six illustrative SRES marker scenarios (coloured lines) and $80^{\text {th }}$ percentile range of recent scenarios published since SRES (post-SRES) (gray shaded area). Dashed lines show the full range of postSRES scenarios. The emissions include $\mathrm{CO}_{2}, \mathrm{CH}_{4}, \mathrm{~N}_{2} \mathrm{O}$ and F-gases. \{WGIII 1.3, 3.2, Figure SPM.4\}

Purchasing Power Parity, PPP) does not appreciably affect the projected emissions, when used consistently. ${ }^{11}$ The differences, if any, are small compared to the uncertainties caused by assumptions on other parameters in the scenarios, e.g. technological change. \{WGIII 3.2, TS.3, SPM\}

\section{SRES scenarios}

SRES refers to the scenarios described in the IPCC Special Report on Emissions Scenarios (SRES, 2000). The SRES scenarios are grouped into four scenario families (A1, A2, B1 and B2) that explore alternative development pathways, covering a wide range of demographic, economic and technological driving forces and resulting GHG emissions. The SRES scenarios do not include additional climate policies above current ones. The emissions projections are widely used in the assessments of future climate change, and their underlying assumptions with respect to socio-economic, demographic and technological change serve as inputs to many recent climate change vulnerability and impact assessments. \{WGI 10.1; WGII 2.4; WGIII TS.1, SPM\}

The A1 storyline assumes a world of very rapid economic growth, a global population that peaks in mid-century and rapid introduction of new and more efficient technologies. A1 is divided into three groups that describe alternative directions of technological change: fossil intensive $(\mathrm{A} 1 \mathrm{FI})$, non-fossil energy resources $(\mathrm{A} 1 \mathrm{~T})$ and a balance across all sources (A1B). B1 describes a convergent world, with the same global population as $A 1$, but with more rapid changes in economic structures toward a service and information economy. B2 describes a world with intermediate population and economic growth, emphasising local solutions to economic, social, and environmental sustainability. A2 describes a very heterogeneous world with high population growth, slow economic development and slow technological change. No likelihood has been attached to any of the SRES scenarios. \{WGIII TS.1, SPM

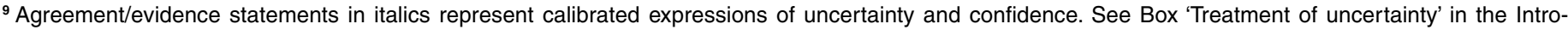
duction for an explanation of these terms.

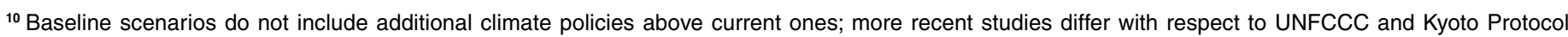
inclusion. Emission pathways of mitigation scenarios are discussed in Topic 5.

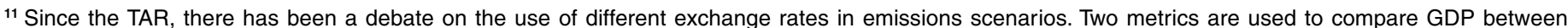

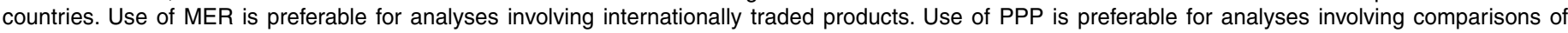

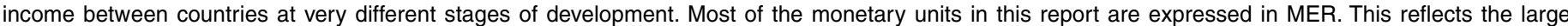
majority of emissions mitigation literature that is calibrated in MER. When monetary units are expressed in PPP, this is denoted by GDP PPP. $_{\text {.WGIII SPM }}$
} 


\subsection{Projections of future changes in climate}

For the next two decades a warming of about $0.2^{\circ} \mathrm{C}$ per decade is projected for a range of SRES emissions scenarios. Even if the concentrations of all GHGs and aerosols had been kept constant at year $\mathbf{2 0 0 0}$ levels, a further warming of about $0.1^{\circ} \mathrm{C}$ per decade would be expected. Afterwards, temperature projections increasingly depend on specific emissions scenarios (Figure 3.2). \{WGI 10.3, 10.7; WGIII 3.2\}

Since the IPCC's first report in 1990, assessed projections have suggested global averaged temperature increases between about 0.15 and $0.3^{\circ} \mathrm{C}$ per decade from 1990 to 2005 . This can now be compared with observed values of about $0.2^{\circ} \mathrm{C}$ per decade, strengthening confidence in near-term projections. \{WGI 1.2, 3.2\}

\subsection{1 $21^{\text {st }}$ century global changes}

Continued GHG emissions at or above current rates would cause further warming and induce many changes in the global climate system during the $21^{\text {st }}$ century that would very likely be larger than those observed during the $20^{\text {th }}$ century. \{WGI 10.3\}

Advances in climate change modelling now enable best estimates and likely assessed uncertainty ranges to be given for projected warming for different emissions scenarios. Table 3.1 shows best estimates and likely ranges for global average surface air warming for the six SRES marker emissions scenarios (including climate-carbon cycle feedbacks). (WGI 10.5\}
Although these projections are broadly consistent with the span quoted in the TAR $\left(1.4\right.$ to $\left.5.8^{\circ} \mathrm{C}\right)$, they are not directly comparable. Assessed upper ranges for temperature projections are larger than in the TAR mainly because the broader range of models now available suggests stronger climate-carbon cycle feedbacks. For the A2 scenario, for example, the climate-carbon cycle feedback increases the corresponding global average warming at 2100 by more than $1^{\circ} \mathrm{C}$. Carbon feedbacks are discussed in Topic 2.3. /WGI 7.3, 10.5, $S P M\}$

Because understanding of some important effects driving sea level rise is too limited, this report does not assess the likelihood, nor provide a best estimate or an upper bound for sea level rise. Model-based projections of global average sea level rise at the end of the $21^{\text {st }}$ century (2090-2099) are shown in Table 3.1. For each scenario, the mid-point of the range in Table 3.1 is within $10 \%$ of the TAR model average for 2090-2099. The ranges are narrower than in the TAR mainly because of improved information about some uncertainties in the projected contributions. ${ }^{12}$ The sea level projections do not include uncertainties in climate-carbon cycle feedbacks nor do they include the full effects of changes in ice sheet flow, because a basis in published literature is lacking. Therefore the upper values of the ranges given are not to be considered upper bounds for sea level rise. The projections include a contribution due to increased ice flow from Greenland and Antarctica at the rates observed for 1993-2003, but these flow rates could increase or decrease in the future. If this contribution were to grow linearly with global average temperature change, the upper ranges of sea level rise for SRES scenarios shown in Table 3.1 would increase by 0.1 to $0.2 \mathrm{~m} \cdot{ }^{13}$ (WGI 10.6, SPM\}

Table 3.1. Projected global average surface warming and sea level rise at the end of the $21^{\text {st }}$ century. $\{$ WGI 10.5, 10.6, Table 10.7, Table SPM.3\}

\begin{tabular}{|c|c|c|c|}
\hline \multirow[b]{2}{*}{ Case } & \multicolumn{2}{|c|}{$\begin{array}{l}\text { Temperature change } \\
\left({ }^{\circ} \mathrm{C} \text { at } 2090-2099 \text { relative to } 1980-1999\right)^{a, d}\end{array}$} & $\begin{array}{l}\text { Sea level rise } \\
\text { ( } m \text { at } 2090-2099 \text { relative to } 1980-1999 \text { ) }\end{array}$ \\
\hline & Best estimate & Likely range & $\begin{array}{l}\text { Model-based range } \\
\text { excluding future rapid dynamical changes in ice flow }\end{array}$ \\
\hline $\begin{array}{l}\text { Constant year } 2000 \\
\text { concentrations }^{\mathrm{b}}\end{array}$ & 0.6 & $0.3-0.9$ & Not available \\
\hline $\begin{array}{l}\text { B1 scenario } \\
\text { A1T scenario } \\
\text { B2 scenario } \\
\text { A1B scenario } \\
\text { A2 scenario } \\
\text { A1FI scenario }\end{array}$ & $\begin{array}{l}1.8 \\
2.4 \\
2.4 \\
2.8 \\
3.4 \\
4.0\end{array}$ & $\begin{array}{l}1.1-2.9 \\
1.4-3.8 \\
1.4-3.8 \\
1.7-4.4 \\
2.0-5.4 \\
2.4-6.4\end{array}$ & $\begin{array}{l}0.18-0.38 \\
0.20-0.45 \\
0.20-0.43 \\
0.21-0.48 \\
0.23-0.51 \\
0.26-0.59\end{array}$ \\
\hline
\end{tabular}

Notes:

a) These estimates are assessed from a hierarchy of models that encompass a simple climate model, several Earth Models of Intermediate Complexity, and a large number of Atmosphere-Ocean General Circulation Models (AOGCMs) as well as observational constraints.

b) Year 2000 constant composition is derived from AOGCMs only.

c) All scenarios above are six SRES marker scenarios. Approximate $\mathrm{CO}_{2}$-eq concentrations corresponding to the computed radiative forcing due to anthropogenic GHGs and aerosols in 2100 (see p. 823 of the WGI TAR) for the SRES B1, AIT, B2, A1B, A2 and A1FI illustrative marker scenarios are about $600,700,800,850,1250$ and $1550 \mathrm{ppm}$, respectively.

d) Temperature changes are expressed as the difference from the period 1980-1999. To express the change relative to the period 1850-1899 add $0.5^{\circ} \mathrm{C}$.

\footnotetext{
${ }^{12}$ TAR projections were made for 2100, whereas the projections for this report are for 2090-2099. The TAR would have had similar ranges to those in Table 3.1 if it had treated uncertainties in the same way.

${ }^{13}$ For discussion of the longer term see Sections 3.2.3 and 5.2.
} 


\subsection{2 $21^{\text {st }}$ century regional changes}

There is now higher confidence than in the TAR in projected patterns of warming and other regional-scale features, including changes in wind patterns, precipitation and some aspects of extremes and sea ice. \{WGI 8.2, 8.3, 8.4, 8.5, 9.4, 9.5, $10.3,11.1\}$

Projected warming in the $21^{\text {st }}$ century shows scenario-independent geographical patterns similar to those observed over the past several decades. Warming is expected to be greatest over land and at most high northern latitudes, and least over the Southern Ocean (near Antarctica) and northern North Atlantic, continuing recent observed trends (Figure 3.2 right panels). \{WGI 10.3, SPM\}

Snow cover area is projected to contract. Widespread increases in thaw depth are projected over most permafrost regions. Sea ice is projected to shrink in both the Arctic and Antarctic under all SRES scenarios. In some projections, Arctic late-summer sea ice disappears almost entirely by the latter part of the $21^{\text {st }}$ century. \{WGI 10.3, 10.6, SPM; WGII 15.3.4\}

It is very likely that hot extremes, heat waves and heavy precipitation events will become more frequent. \{SYR Table 3.2; WGI 10.3, SPM\}

Based on a range of models, it is likely that future tropical cyclones (typhoons and hurricanes) will become more intense, with larger peak wind speeds and more heavy precipitation associated with ongoing increases of tropical sea-surface temperatures. There is less confidence in projections of a global decrease in numbers of tropical cyclones. The apparent increase in the proportion of very intense storms since 1970 in some regions is much larger than simulated by current models for that period. \{WGI 3.8, 9.5, 10.3, SPM\}

Extra-tropical storm tracks are projected to move poleward, with consequent changes in wind, precipitation and temperature patterns, continuing the broad pattern of observed trends over the last halfcentury. \{WGI 3.6, 10.3, SPM \}

Since the TAR there is an improving understanding of projected patterns of precipitation. Increases in the amount of precipitation are very likely in high-latitudes, while decreases are likely in most subtropical land regions (by as much as about $20 \%$ in the A1B scenario in 2100, Figure 3.3), continuing observed patterns in recent trends. $\{$ WGI 3.3, 8.3, 9.5, 10.3, 11.2-11.9, SPM \}

\subsubsection{Changes beyond the $21^{\text {st }}$ century}

Anthropogenic warming and sea level rise would continue for centuries due to the time scales associated with climate processes and feedbacks, even if GHG concentrations were to be stabilised. \{WGI 10.4, 10.5, 10.7, SPM $\}$

If radiative forcing were to be stabilised, keeping all the radiative forcing agents constant at B1 or A1B levels in 2100, model experiments show that a further increase in global average temperature of about $0.5^{\circ} \mathrm{C}$ would still be expected by 2200. In addition, thermal expansion alone would lead to 0.3 to $0.8 \mathrm{~m}$ of sea level rise by 2300 (relative to 1980-1999). Thermal expansion would continue for many centuries, due to the time required to transport heat into the deep ocean. \{WGI 10.7, SPM\}

Atmosphere-Ocean General Circulation Model projections of surface warming
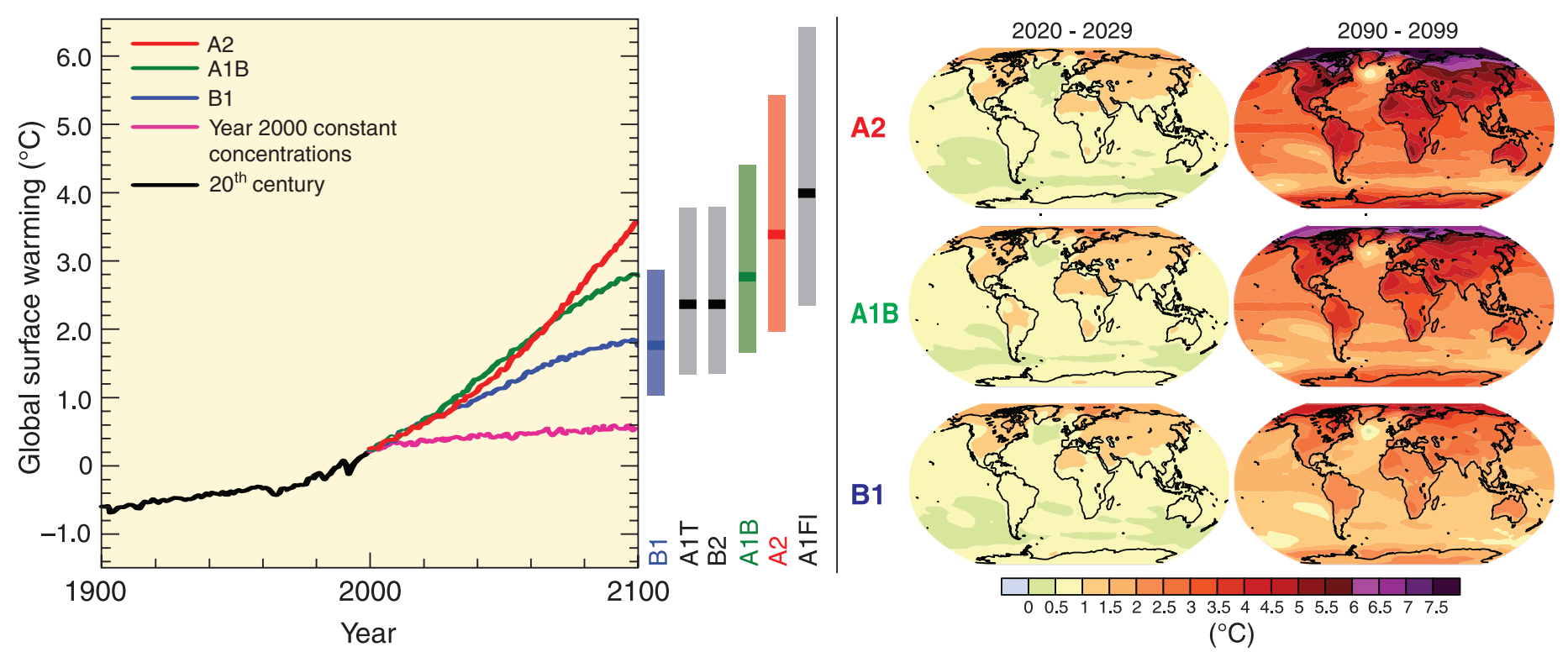

Figure 3.2. Left panel: Solid lines are multi-model global averages of surface warming (relative to 1980-1999) for the SRES scenarios A2, A1B and B1, shown as continuations of the $20^{\text {th }}$ century simulations. The orange line is for the experiment where concentrations were held constant at year 2000 values. The bars in the middle of the figure indicate the best estimate (solid line within each bar) and the likely range assessed for the six SRES marker scenarios at 2090-2099 relative to 1980-1999. The assessment of the best estimate and likely ranges in the bars includes the Atmosphere-Ocean General Circulation Models (AOGCMs) in the left part of the figure, as well as results from a hierarchy of independent models and observational constraints. Right panels: Projected surface temperature changes for the early and late $21^{\text {st }}$ century relative to the period 1980-1999. The panels show the multi-AOGCM average projections for the A2 (top), A1B (middle) and B1 (bottom) SRES scenarios averaged over decades 2020-2029 (left) and 2090-2099 (right). \{WGI 10.4, 10.8, Figures 10.28, 10.29, SPM\} 
Multi-model projected patterns of precipitation changes
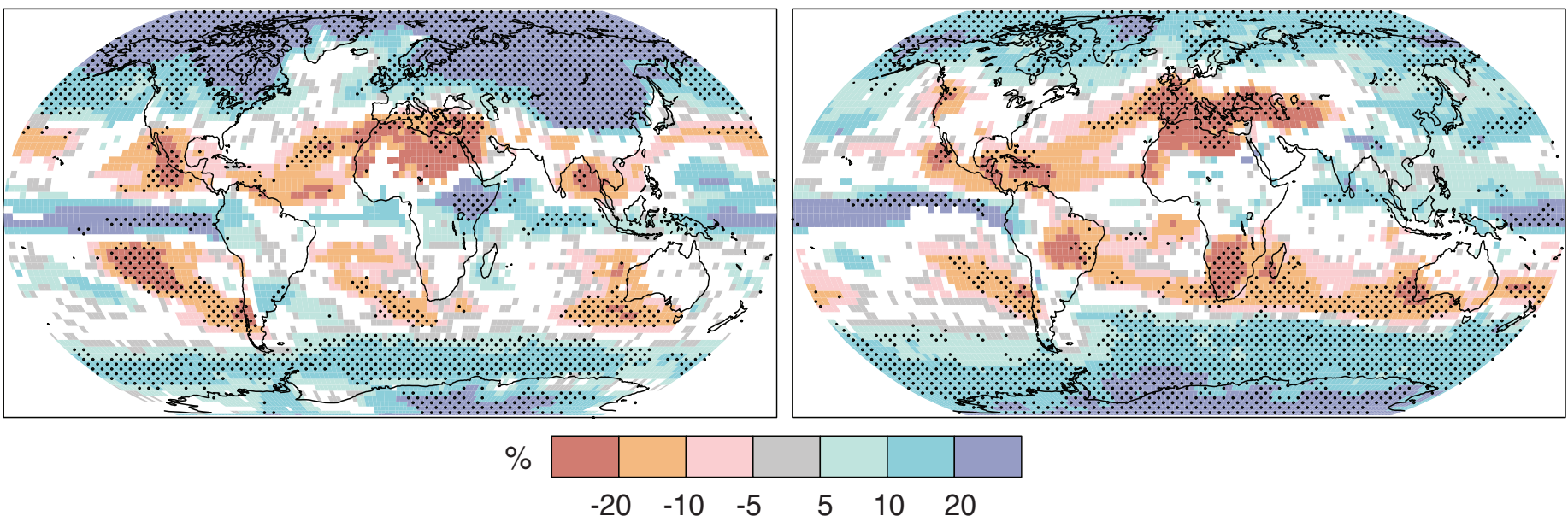

Figure 3.3. Relative changes in precipitation (in percent) for the period 2090-2099, relative to 1980-1999. Values are multi-model averages based on the SRES A1B scenario for December to February (left) and June to August (right). White areas are where less than $66 \%$ of the models agree in the sign of the change and stippled areas are where more than $90 \%$ of the models agree in the sign of the change. \{WGI Figure 10.9, SPM\}

Contraction of the Greenland ice sheet is projected to continue to contribute to sea level rise after 2100. Current models suggest ice mass losses increase with temperature more rapidly than gains due to increased precipitation and that the surface mass balance becomes negative (net ice loss) at a global average warming (relative to pre-industrial values) in excess of 1.9 to $4.6^{\circ} \mathrm{C}$. If such a negative surface mass balance were sustained for millennia, that would lead to virtually complete elimination of the Greenland ice sheet and a resulting contribution to sea level rise of about $7 \mathrm{~m}$. The corresponding future temperatures in Greenland $\left(1.9\right.$ to $4.6^{\circ} \mathrm{C}$ global) are comparable to those inferred for the last interglacial period 125,000 years ago, when palaeoclimatic information suggests reductions of polar land ice extent and 4 to $6 \mathrm{~m}$ of sea level rise. \{WGI 6.4, 10.7, SPM\}

Dynamical processes related to ice flow - which are not included in current models but suggested by recent observations - could increase the vulnerability of the ice sheets to warming, increasing future sea level rise. Understanding of these processes is limited and there is no consensus on their magnitude. \{WGI 4.6, 10.7, $S P M\}$

Current global model studies project that the Antarctic ice sheet will remain too cold for widespread surface melting and gain mass due to increased snowfall. However, net loss of ice mass could occur if dynamical ice discharge dominates the ice sheet mass balance. $\{$ WGI 10.7, SPM \}

Both past and future anthropogenic $\mathrm{CO}_{2}$ emissions will continue to contribute to warming and sea level rise for more than a millennium, due to the time scales required for the removal of this gas from the atmosphere. \{WGI 7.3, 10.3, Figure 7.12, Figure 10.35, SPM\}

Estimated long-term (multi-century) warming corresponding to the six AR4 WG III stabilisation categories is shown in Figure 3.4.

\section{Estimated multi-century warming relative to 1980-1999 for AR4 stabilisation categories}

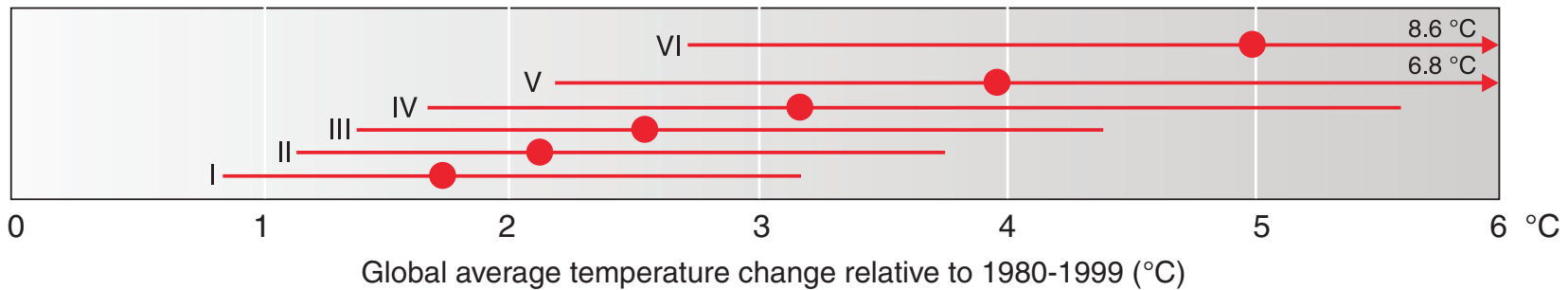

Figure 3.4. Estimated long-term (multi-century) warming corresponding to the six AR4 WG III stabilisation categories (Table 5.1). The temperature scale has been shifted by $-0.5^{\circ} \mathrm{C}$ compared to Table 5.1 to account approximately for the warming between pre-industrial and 1980-1999. For most stabilisation levels global average temperature is approaching the equilibrium level over a few centuries. For GHG emissions scenarios that lead to stabilisation at levels comparable to SRES B1 and A1B by 2100 (600 and 850 ppm CO -eq; category IV and V), assessed models project that about 65 to $70 \%$ of the estimated global equilibrium temperature increase, assuming a climate sensitivity of $3^{\circ} \mathrm{C}$, would be realised at the time of stabilisation. For the much lower stabilisation scenarios (category I and II, Figure 5.1), the equilibrium temperature may be reached earlier. \{WGI 10.7.2\} 
3.3 Impacts of future climate changes

More specific information is now available across a wide range of systems and sectors concerning the nature of future impacts, including some fields not covered in previous assessments. \{WGII TS.4, SPM\}

The following is a selection of key findings ${ }^{14}$ regarding the impacts of climate change on systems, sectors and regions, as well as some findings on vulnerability ${ }^{15}$, for the range of climate changes projected over the $21^{\text {st }}$ century. Unless otherwise stated, the confidence level in the projections is high. Global average temperature increases are given relative to 1980-1999. Additional information on impacts can be found in the WG II report. \{WGII SPM\}

\subsubsection{Impacts on systems and sectors}

\section{Ecosystems}

- The resilience of many ecosystems is likely to be exceeded this century by an unprecedented combination of climate change, associated disturbances (e.g. flooding, drought, wildfire, insects, ocean acidification) and other global change drivers (e.g. landuse change, pollution, fragmentation of natural systems, overexploitation of resources). \{WGII 4.1-4.6, SPM \}

- Over the course of this century, net carbon uptake by terrestrial ecosystems is likely to peak before mid-century and then weaken or even reverse ${ }^{16}$, thus amplifying climate change. (WGII 4.ES, Figure 4.2, SPM

- Approximately 20 to $30 \%$ of plant and animal species assessed so far are likely to be at increased risk of extinction if increases in global average temperature exceed 1.5 to $2.5^{\circ} \mathrm{C}$ (medium confidence). \{WGII 4.ES, Figure 4.2, SPM\}

- For increases in global average temperature exceeding 1.5 to $2.5^{\circ} \mathrm{C}$ and in concomitant atmospheric $\mathrm{CO}_{2}$ concentrations, there are projected to be major changes in ecosystem structure and function, species' ecological interactions and shifts in species' geographical ranges, with predominantly negative consequences for biodiversity and ecosystem goods and services, e.g. water and food supply. \{WGII 4.4, Box TS.6, SPM \}

\section{Food}

- Crop productivity is projected to increase slightly at mid- to high latitudes for local mean temperature increases of up to 1 to $3^{\circ} \mathrm{C}$ depending on the crop, and then decrease beyond that in some regions (medium confidence). \{WGII 5.4, SPM\}

- At lower latitudes, especially in seasonally dry and tropical regions, crop productivity is projected to decrease for even small local temperature increases $\left(1\right.$ to $\left.2^{\circ} \mathrm{C}\right)$, which would increase the risk of hunger (medium confidence). \{WGII 5.4, SPM\}

- Globally, the potential for food production is projected to increase with increases in local average temperature over a range of 1 to $3^{\circ} \mathrm{C}$, but above this it is projected to decrease (medium confidence). \{WGII 5.4, 5.5, SPM\}

\section{Coasts}

- Coasts are projected to be exposed to increasing risks, including coastal erosion, due to climate change and sea level rise. The effect will be exacerbated by increasing human-induced pressures on coastal areas (very high confidence). \{WGII 6.3, 6.4, $S P M\}$

- By the 2080s, many millions more people than today are projected to experience floods every year due to sea level rise. The numbers affected will be largest in the densely populated and low-lying megadeltas of Asia and Africa while small islands are especially vulnerable (very high confidence). \{WGII 6.4, 6.5, Table 6.11, SPM\}

\section{Industry, settlements and society}

- The most vulnerable industries, settlements and societies are generally those in coastal and river flood plains, those whose economies are closely linked with climate-sensitive resources and those in areas prone to extreme weather events, especially where rapid urbanisation is occurring. \{WGII 7.1, 7.3, 7.4, 7.5, SPM

- Poor communities can be especially vulnerable, in particular those concentrated in high-risk areas. \{WGII 7.2, 7.4, 5.4, SPM\}

\section{Health}

- The health status of millions of people is projected to be affected through, for example, increases in malnutrition; increased deaths, diseases and injury due to extreme weather events; increased burden of diarrhoeal diseases; increased frequency of cardio-respiratory diseases due to higher concentrations of ground-level ozone in urban areas related to climate change; and the altered spatial distribution of some infectious diseases. \{WGI 7.4, Box 7.4; WGII 8.ES, 8.2, 8.4, SPM\}

- Climate change is projected to bring some benefits in temperate areas, such as fewer deaths from cold exposure, and some mixed effects such as changes in range and transmission potential of malaria in Africa. Overall it is expected that benefits will be outweighed by the negative health effects of rising temperatures, especially in developing countries. \{WGII 8.4, 8.7, 8ES, SPM\}

- Critically important will be factors that directly shape the health of populations such as education, health care, public health initiatives, and infrastructure and economic development. \{WGII 8.3, SPM $\}$

\section{Water}

- Water impacts are key for all sectors and regions. These are discussed below in the Box 'Climate change and water'.

\footnotetext{
${ }^{14}$ Criteria of choice: magnitude and timing of impact, confidence in the assessment, representative coverage of the system, sector and region.

${ }^{15}$ Vulnerability to climate change is the degree to which systems are susceptible to, and unable to cope with, adverse impacts.

${ }^{16}$ Assuming continued GHG emissions at or above current rates and other global changes including land-use changes.
} 


\section{Climate change and water}

Climate change is expected to exacerbate current stresses on water resources from population growth and economic and land-use change, including urbanisation. On a regional scale, mountain snow pack, glaciers and small ice caps play a crucial role in freshwater availability. Widespread mass losses from glaciers and reductions in snow cover over recent decades are projected to accelerate throughout the $21^{\text {st }}$ century, reducing water availability, hydropower potential, and changing seasonality of flows in regions supplied by meltwater from major mountain ranges (e.g. Hindu-Kush, Himalaya, Andes), where more than one-sixth of the world population currently lives. \{WGI 4.1, 4.5; WGII 3.3, 3.4, 3.5\}

Changes in precipitation (Figure 3.3) and temperature (Figure 3.2) lead to changes in runoff (Figure 3.5) and water availability. Runoff is projected with high confidence to increase by 10 to $40 \%$ by mid-century at higher latitudes and in some wet tropical areas, including populous areas in East and South-East Asia, and decrease by 10 to $30 \%$ over some dry regions at mid-latitudes and dry tropics, due to decreases in rainfall and higher rates of evapotranspiration. There is also high confidence that many semi-arid areas (e.g. the Mediterranean Basin, western United States, southern Africa and north-eastern Brazil) will suffer a decrease in water resources due to climate change. Drought-affected areas are projected to increase in extent, with the potential for adverse impacts on multiple sectors, e.g. agriculture, water supply, energy production and health. Regionally, large increases in irrigation water demand as a result of climate changes are projected. \{WGI 10.3, 11.2-11.9; WGIl 3.4, 3.5, Figure 3.5, TS.4.1, Box TS.5, SPM\}

The negative impacts of climate change on freshwater systems outweigh its benefits (high confidence). Areas in which runoff is projected to decline face a reduction in the value of the services provided by water resources (very high confidence). The beneficial impacts of increased annual runoff in some areas are likely to be tempered by negative effects of increased precipitation variability and seasonal runoff shifts on water supply, water quality and flood risk. \{WGII 3.4, 3.5, TS.4.1\}

Available research suggests a significant future increase in heavy rainfall events in many regions, including some in which the mean rainfall is projected to decrease. The resulting increased flood risk poses challenges to society, physical infrastructure and water quality. It is likely that up to $20 \%$ of the world population will live in areas where river flood potential could increase by the 2080 s. Increases in the frequency and severity of floods and droughts are projected to adversely affect sustainable development. Increased temperatures will further affect the physical, chemical and biological properties of freshwater lakes and rivers, with predominantly adverse impacts on many individual freshwater species, community composition and water quality. In coastal areas, sea level rise will exacerbate water resource constraints due to increased salinisation of groundwater supplies. \{WGI 11.2-11.9; WGII 3.2, 3.3, 3.4, 4.4\}

\section{Projections and model consistency of relative changes in runoff by the end of the 21st century}

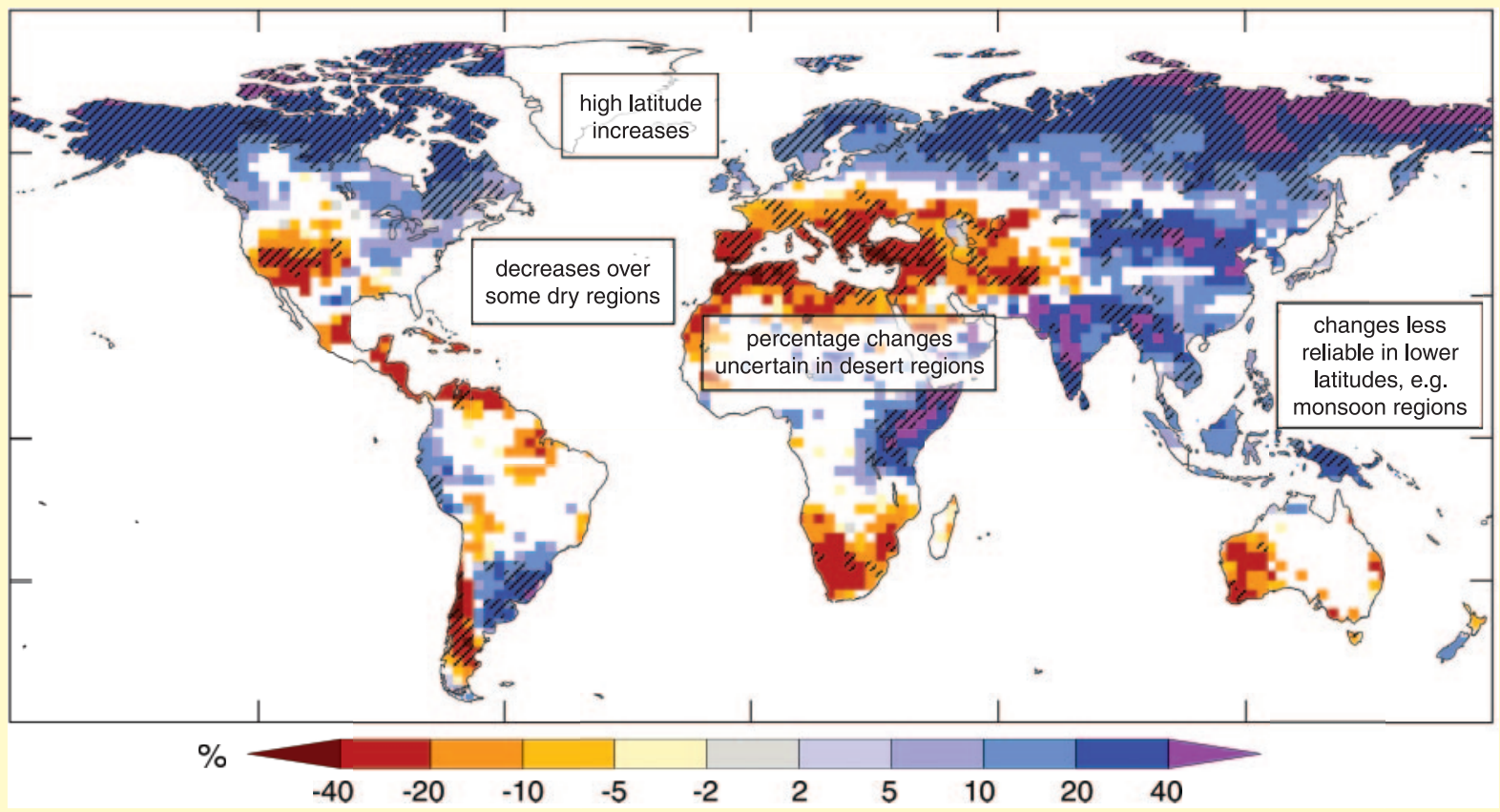

Figure 3.5. Large-scale relative changes in annual runoff (water availability, in percent) for the period 2090-2099, relative to 1980-1999. Values represent the median of 12 climate models using the SRES A1B scenario. White areas are where less than $66 \%$ of the 12 models agree on the sign of change and hatched areas are where more than $90 \%$ of models agree on the sign of change. The quality of the simulation of the observed large-scale $20^{\text {th }}$ century runoff is used as a basis for selecting the 12 models from the multi-model ensemble. The global map of annual runoff illustrates a large scale and is not intended to refer to smaller temporal and spatial scales. In areas where rainfall and runoff is very low (e.g. desert areas), small changes in runoff can lead to large percentage changes. In some regions, the sign of projected changes in runoff differs from recently observed trends. In some areas with projected increases in runoff, different seasonal effects are expected, such as increased wet season runoff and decreased dry season runoff. Studies using results from few climate models can be considerably different from the results presented here. $\{$ WGII Figure 3.4 , adjusted to match the assumptions of Figure SYR 3.3; WGII 3.3.1, 3.4.1, 3.5.1\} 


\section{Studies since the TAR have enabled more systematic un- derstanding of the timing and magnitude of impacts related to differing amounts and rates of climate change. \{WGII SPM\}}

Examples of this new information for systems and sectors are presented in Figure 3.6. The upper panel shows impacts increasing with increasing temperature change. Their estimated magnitude and timing is also affected by development pathways (lower panel). \{WGII SPM\}

Depending on circumstances, some of the impacts shown in Figure 3.6 could be associated with 'key vulnerabilities', based on a number of criteria in the literature (magnitude, timing, persistence/ reversibility, the potential for adaptation, distributional aspects, likelihood and 'importance' of the impacts) (see Topic 5.2). \{WGII SPM\}

\subsubsection{Impacts on regions ${ }^{17}$}

Africa

- By 2020, between 75 and 250 million of people are projected to be exposed to increased water stress due to climate change. \{WGII 9.4, SPM\}

- By 2020, in some countries, yields from rain-fed agriculture could be reduced by up to $50 \%$. Agricultural production, including access to food, in many African countries is projected to be severely compromised. This would further adversely affect food security and exacerbate malnutrition. \{WGII 9.4, SPM\}

- Towards the end of the $21^{\text {st }}$ century, projected sea level rise will affect low-lying coastal areas with large populations. The cost of adaptation could amount to at least 5 to $10 \%$ of GDP. \{WGII 9.4, SPM\}

- By 2080, an increase of 5 to $8 \%$ of arid and semi-arid land in Africa is projected under a range of climate scenarios (high confidence). (WGII Box TS.6, 9.4.4\}

\section{Asia}

- By the 2050s, freshwater availability in Central, South, East and South-East Asia, particularly in large river basins, is projected to decrease. \{WGII 10.4, SPM\}

- Coastal areas, especially heavily populated megadelta regions in South, East and South-East Asia, will be at greatest risk due to increased flooding from the sea and, in some megadeltas, flooding from the rivers. \{WGII 10.4, SPM \}

- Climate change is projected to compound the pressures on natural resources and the environment associated with rapid urbanisation, industrialisation and economic development. \{WGII 10.4, SPM\}

- Endemic morbidity and mortality due to diarrhoeal disease primarily associated with floods and droughts are expected to rise in East, South and South-East Asia due to projected changes in the hydrological cycle. (WGII 10.4, SPM\}

\section{Australia and New Zealand}

- By 2020, significant loss of biodiversity is projected to occur in some ecologically rich sites, including the Great Barrier Reef and Queensland Wet Tropics. \{WGII 11.4, SPM\}
- By 2030, water security problems are projected to intensify in southern and eastern Australia and, in New Zealand, in Northland and some eastern regions. \{WGII 11.4, SPM\}

- By 2030, production from agriculture and forestry is projected to decline over much of southern and eastern Australia, and over parts of eastern New Zealand, due to increased drought and fire. However, in New Zealand, initial benefits are projected in some other regions. \{WGII 11.4, SPM \}

- By 2050, ongoing coastal development and population growth in some areas of Australia and New Zealand are projected to exacerbate risks from sea level rise and increases in the severity and frequency of storms and coastal flooding. (WGII 11.4, $S P M\}$

\section{Europe}

- Climate change is expected to magnify regional differences in Europe's natural resources and assets. Negative impacts will include increased risk of inland flash floods and more frequent coastal flooding and increased erosion (due to storminess and sea level rise). (WGII 12.4, SPM \}

- Mountainous areas will face glacier retreat, reduced snow cover and winter tourism, and extensive species losses (in some areas up to $60 \%$ under high emissions scenarios by 2080). (WGII 12.4, $S P M\}$

- In southern Europe, climate change is projected to worsen conditions (high temperatures and drought) in a region already vulnerable to climate variability, and to reduce water availability, hydropower potential, summer tourism and, in general, crop productivity. \{WGII 12.4, SPM \}

- Climate change is also projected to increase the health risks due to heat waves and the frequency of wildfires. [WGII 12.4, $S P M\}$

\section{Latin America}

- By mid-century, increases in temperature and associated decreases in soil water are projected to lead to gradual replacement of tropical forest by savanna in eastern Amazonia. Semiarid vegetation will tend to be replaced by arid-land vegetation. \{WGII 13.4, SPM\}

- There is a risk of significant biodiversity loss through species extinction in many areas of tropical Latin America. \{WGII 13.4, $S P M\}$

- Productivity of some important crops is projected to decrease and livestock productivity to decline, with adverse consequences for food security. In temperate zones, soybean yields are projected to increase. Overall, the number of people at risk of hunger is projected to increase (medium confidence). \{WGII 13.4, Box TS.6\}

- Changes in precipitation patterns and the disappearance of glaciers are projected to significantly affect water availability for human consumption, agriculture and energy generation. \{WGII 13.4, SPM\}

\footnotetext{
${ }_{17}$ Unless stated explicitly, all entries are from WG II SPM text, and are either very high confidence or high confidence statements, reflecting different sectors (agriculture, ecosystems, water, coasts, health, industry and settlements). The WG II SPM refers to the source of the statements, timelines and temperatures. The magnitude and timing of impacts that will ultimately be realised will vary with the amount and rate of climate change, emissions scenarios, development pathways and adaptation.
} 
Examples of impacts associated with global average temperature change

(Impacts will vary by extent of adaptation, rate of temperature change and socio-economic pathway)

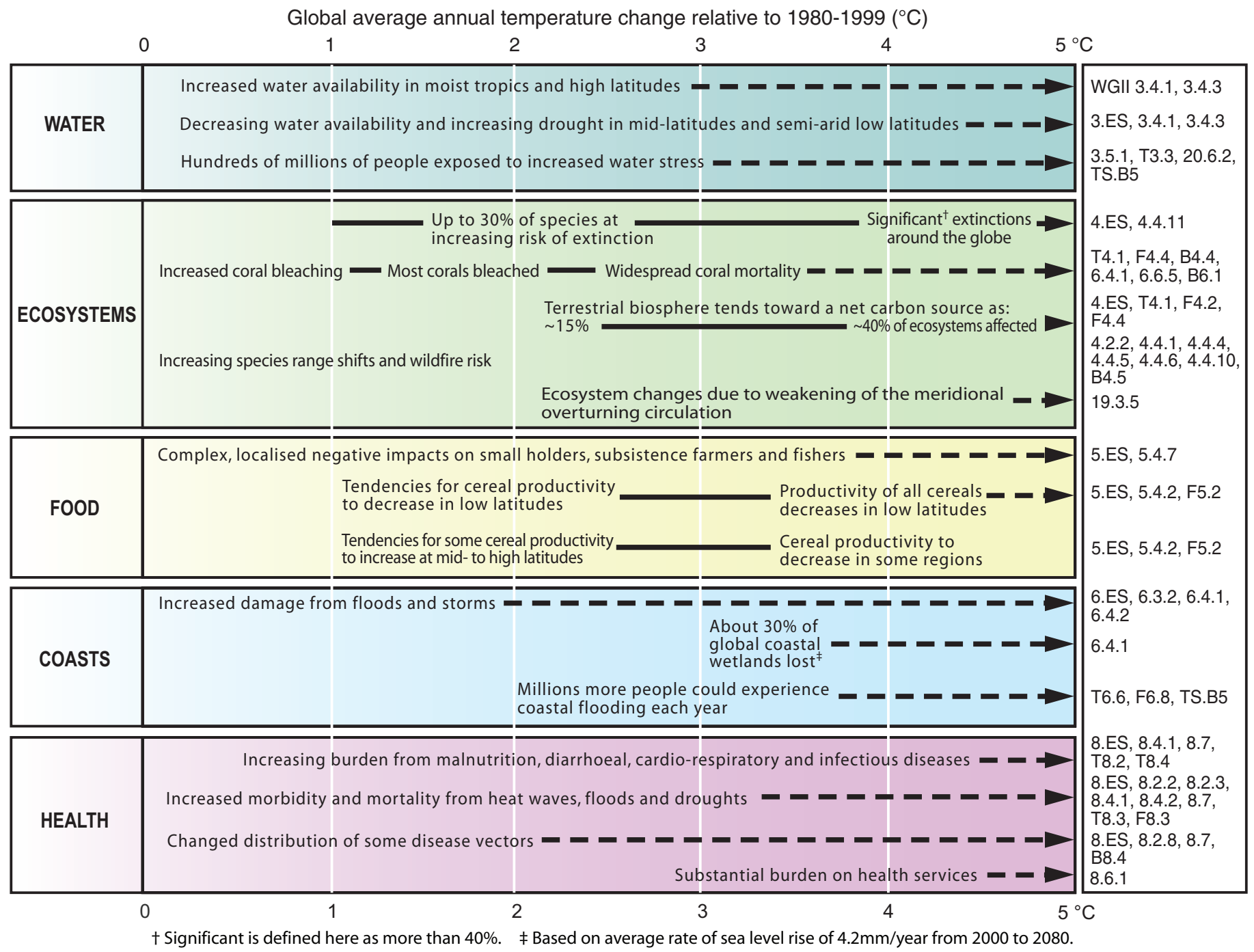

Warming by 2090-2099 relative to $1980-1999$ for non-mitigation scenarios

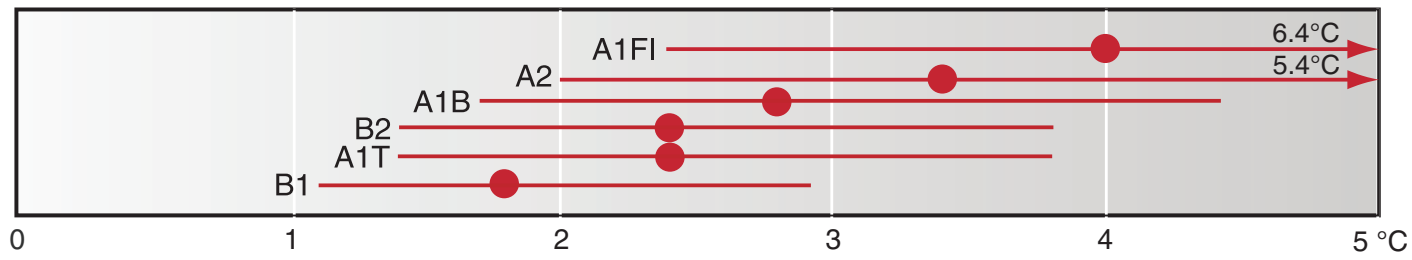

Figure 3.6. Examples of impacts associated with global average temperature change. Upper panel: Illustrative examples of global impacts projected for climate changes (and sea level and atmospheric $\mathrm{CO}_{2}$ where relevant) associated with different amounts of increase in global average surface temperature in the $21^{\text {st }}$ century. The black lines link impacts; broken-line arrows indicate impacts continuing with increasing temperature. Entries are placed so that the left-hand side of text indicates the approximate level of warming that is associated with the onset of a given impact. Quantitative entries for water scarcity and flooding represent the additional impacts of climate change relative to the conditions projected across the range of SRES scenarios A1FI, A2, B1 and B2. Adaptation to climate change is not included in these estimations. Confidence levels for all statements are high. The upper right panel gives the WG II references for the statements made in the upper left panel.* Lower panel: Dots and bars indicate the best estimate and likely ranges of warming assessed for the six SRES marker scenarios for 2090-2099 relative to 1980-1999. \{WGI Figure SPM.5, 10.7; WGIl Figure SPM.2; WGIII Table TS.2, Table 3.10\}

*Where ES = Executive Summary, $\mathrm{T}=$ Table, B = Box and F = Figure. Thus B4.5 indicates Box 4.5 in Chapter 4 and 3.5.1 indicates Section 3.5.1 in Chapter 3. 


\section{North America}

- Warming in western mountains is projected to cause decreased snowpack, more winter flooding and reduced summer flows, exacerbating competition for over-allocated water resources. \{WGII 14.4, SPM\}

- In the early decades of the century, moderate climate change is projected to increase aggregate yields of rain-fed agriculture by 5 to $20 \%$, but with important variability among regions. Major challenges are projected for crops that are near the warm end of their suitable range or which depend on highly utilised water resources. \{WGII 14.4, SPM \}

- Cities that currently experience heat waves are expected to be further challenged by an increased number, intensity and duration of heat waves during the course of the century, with potential for adverse health impacts. \{WGII 14.4, SPM\}

- Coastal communities and habitats will be increasingly stressed by climate change impacts interacting with development and pollution. \{WGII 14.4, SPM \}

\section{Polar Regions}

- The main projected biophysical effects are reductions in thickness and extent of glaciers, ice sheets and sea ice, and changes in natural ecosystems with detrimental effects on many organisms including migratory birds, mammals and higher predators. \{WGII 15.4, SPM\}

- For human communities in the Arctic, impacts, particularly those resulting from changing snow and ice conditions, are projected to be mixed. \{WGII 15.4, SPM \}

- Detrimental impacts would include those on infrastructure and traditional indigenous ways of life. \{WGII 15.4, SPM \}

- In both polar regions, specific ecosystems and habitats are projected to be vulnerable, as climatic barriers to species invasions are lowered. \{WGII 15.4, SPM

\section{Small Islands}

- Sea level rise is expected to exacerbate inundation, storm surge, erosion and other coastal hazards, thus threatening vital infrastructure, settlements and facilities that support the livelihood of island communities. \{WGII 16.4, SPM\}

- Deterioration in coastal conditions, for example through erosion of beaches and coral bleaching, is expected to affect local resources. \{WGII 16.4, SPM \}

- By mid-century, climate change is expected to reduce water resources in many small islands, e.g. in the Caribbean and $\mathrm{Pa}-$ cific, to the point where they become insufficient to meet demand during low-rainfall periods. \{WGII 16.4, SPM \}

- With higher temperatures, increased invasion by non-native species is expected to occur, particularly on mid- and high-latitude islands. \{WGII 16.4, SPM\}

\subsubsection{Especially affected systems, sectors and regions}

Some systems, sectors and regions are likely to be especially affected by climate change. ${ }^{18}$ \{WGII TS.4.5\}

Systems and sectors: \{WGII TS.4.5\}

- particular ecosystems:

- terrestrial: tundra, boreal forest and mountain regions because of sensitivity to warming; mediterranean-type ecosystems because of reduction in rainfall; and tropical rainforests where precipitation declines

- coastal: mangroves and salt marshes, due to multiple stresses - marine: coral reefs due to multiple stresses; the sea-ice biome because of sensitivity to warming

- water resources in some dry regions at mid-latitudes ${ }^{19}$ and in the dry tropics, due to changes in rainfall and evapotranspiration, and in areas dependent on snow and ice melt

- agriculture in low latitudes, due to reduced water availability

- low-lying coastal systems, due to threat of sea level rise and increased risk from extreme weather events

- human health in populations with low adaptive capacity.

Regions: \{WGII TS.4.5\}

- the Arctic, because of the impacts of high rates of projected warming on natural systems and human communities

- Africa, because of low adaptive capacity and projected climate change impacts

- small islands, where there is high exposure of population and infrastructure to projected climate change impacts

- Asian and African megadeltas, due to large populations and high exposure to sea level rise, storm surges and river flooding.

Within other areas, even those with high incomes, some people (such as the poor, young children and the elderly) can be particularly at risk, and also some areas and some activities. \{WGII 7.1, 7.2, $7.4,8.2,8.4$, TS.4.5\}

\subsubsection{Ocean acidification}

The uptake of anthropogenic carbon since 1750 has led to the ocean becoming more acidic with an average decrease in $\mathrm{pH}$ of 0.1 units. Increasing atmospheric $\mathrm{CO}_{2}$ concentrations lead to further acidification. Projections based on SRES scenarios give a reduction in average global surface ocean $\mathrm{pH}$ of between 0.14 and 0.35 units over the $21^{\text {st }}$ century. While the effects of observed ocean acidification on the marine biosphere are as yet undocumented, the progressive acidification of oceans is expected to have negative impacts on marine shell-forming organisms (e.g. corals) and their dependent species. \{WGI SPM; WGII SPM\}

\subsubsection{Extreme events}

Altered frequencies and intensities of extreme weather, together with sea level rise, are expected to have mostly adverse effects on natural and human systems (Table 3.2). \{WGII SPM\}

Examples for selected extremes and sectors are shown in Table 3.2.

\footnotetext{
${ }^{18}$ Identified on the basis of expert judgement of the assessed literature and considering the magnitude, timing and projected rate of climate change, sensitivity and adaptive capacity.

19 Including arid and semi-arid regions.
} 
Table 3.2. Examples of possible impacts of climate change due to changes in extreme weather and climate events, based on projections to the mid- to late $21^{\text {st }}$ century. These do not take into account any changes or developments in adaptive capacity. The likelihood estimates in column two relate to the phenomena listed in column one. \{WGII Table SPM.1\}

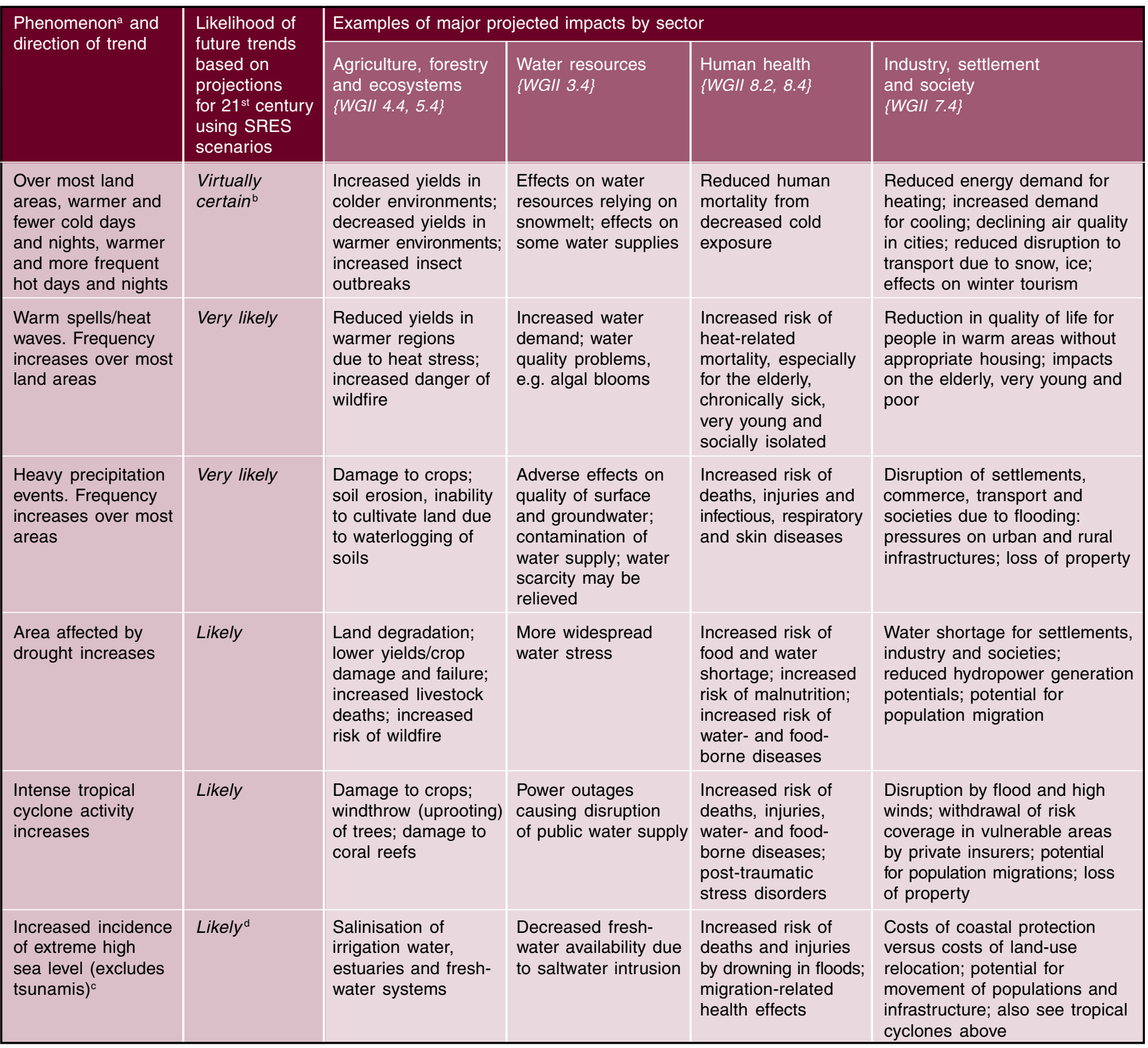

Notes:

a) See WGI Table 3.7 for further details regarding definitions.

b) Warming of the most extreme days and nights each year.

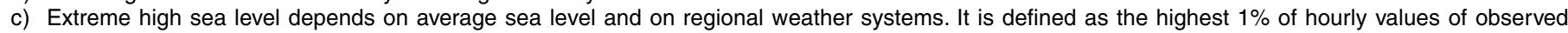
sea level at a station for a given reference period.

d) In all scenarios, the projected global average sea level at 2100 is higher than in the reference period. The effect of changes in regional weather systems on sea level extremes has not been assessed. \{WGI 10.6\}

\subsection{Risk of abrupt or irreversible changes}

\section{Anthropogenic warming could lead to some impacts that are abrupt or irreversible, depending upon the rate and magnitude of the climate change. \{WGII 12.6, 19.3, 19.4, SPM\}}

Abrupt climate change on decadal time scales is normally thought of as involving ocean circulation changes. In addition on longer time scales, ice sheet and ecosystem changes may also play a role. If a large-scale abrupt climate change were to occur, its impact could be quite high (see Topic 5.2). (WGI 8.7, 10.3, 10.7; WGII 4.4, 19.3\}

Partial loss of ice sheets on polar land and/or the thermal expansion of seawater over very long time scales could imply metres of sea level rise, major changes in coastlines and inundation of low-lying areas, with greatest effects in river deltas and low-lying 
islands. Current models project that such changes would occur over very long time scales (millennial) if a global temperature increase of 1.9 to $4.6^{\circ} \mathrm{C}$ (relative to pre-industrial) were to be sustained. Rapid sea level rise on century time scales cannot be excluded. \{SYR 3.2.3; WGI 6.4, 10.7; WGII 19.3, SPM\}

Climate change is likely to lead to some irreversible impacts. There is medium confidence that approximately 20 to $30 \%$ of species assessed so far are likely to be at increased risk of extinction if increases in global average warming exceed 1.5 to $2.5^{\circ} \mathrm{C}$ (relative to 1980-1999). As global average temperature increase exceeds about $3.5^{\circ} \mathrm{C}$, model projections suggest significant extinctions (40 to $70 \%$ of species assessed) around the globe. \{WGII 4.4, Figure SPM.2\}
Based on current model simulations, it is very likely that the meridional overturning circulation (MOC) of the Atlantic Ocean will slow down during the $21^{\text {st }}$ century; nevertheless temperatures in the region are projected to increase. It is very unlikely that the MOC will undergo a large abrupt transition during the $21^{\text {st }}$ century. Longer-term changes in the MOC cannot be assessed with confidence. \{WGI 10.3, 10.7; WGII Figure, Table TS.5, SPM.2\}

Impacts of large-scale and persistent changes in the MOC are likely to include changes in marine ecosystem productivity, fisheries, ocean $\mathrm{CO}_{2}$ uptake, oceanic oxygen concentrations and terrestrial vegetation. Changes in terrestrial and ocean $\mathrm{CO}_{2}$ uptake may feed back on the climate system. \{WGII 12.6, 19.3, Figure SPM.2\} 
Adaptation and mitigation options and responses, and the inter-relationship with sustainable development, at global and regional levels 


\subsection{Responding to climate change}

Societies can respond to climate change by adapting to its impacts and by reducing GHG emissions (mitigation), thereby reducing the rate and magnitude of change. This Topic focuses on adaptation and mitigation options that can be implemented over the next two to three decades, and their inter-relationship with sustainable development. These responses can be complementary. Topic 5 addresses their complementary roles on a more conceptual basis over a longer timeframe.

The capacity to adapt and mitigate is dependent on socio-economic and environmental circumstances and the availability of information and technology $y^{20}$. However, much less information is available about the costs and effectiveness of adaptation measures than about mitigation measures. \{WGII 17.1, 17.3; WGIII 1.2\}

\subsection{Adaptation options}

Adaptation can reduce vulnerability, both in the short and the long term. \{WGII 17.2, 18.1, 18.5, 20.3, 20.8\}

Vulnerability to climate change can be exacerbated by other stresses. These arise from, for example, current climate hazards, poverty, unequal access to resources, food insecurity, trends in economic globalisation, conflict and incidence of diseases such as HIV/ AIDS. \{WGII 7.2, 7.4, 8.3, 17.3, 20.3, 20.4, 20.7, SPM\}

Societies across the world have a long record of adapting and reducing their vulnerability to the impacts of weather- and climaterelated events such as floods, droughts and storms. Nevertheless, additional adaptation measures will be required at regional and local levels to reduce the adverse impacts of projected climate change and variability, regardless of the scale of mitigation undertaken over the next two to three decades. However, adaptation alone is not expected to cope with all the projected effects of climate change, especially not over the long term as most impacts increase in magnitude. \{WGII 17.2, SPM; WGIII 1.2\}

A wide array of adaptation options is available, but more extensive adaptation than is currently occurring is required to reduce vulnerability to climate change. There are barriers, limits and costs, which are not fully understood. Some planned adaptation is already occurring on a limited basis. Table 4.1 provides examples of planned adaptation options by sector. Many adaptation actions have multiple drivers, such as economic development and poverty alleviation, and are embedded within broader development, sectoral, regional and local planning initiatives such as water resources planning, coastal defence and disaster risk reduction strategies. Examples of this approach are the Bangladesh National Water Management Plan and the coastal defence plans of The Netherlands and Norway, which incorporate specific climate change scenarios. \{WGII 1.3, 5.5.2, 11.6, 17.2\}

Comprehensive estimates of the costs and benefits of adaptation at the global level are limited in number. However, the number of adaptation cost and benefit estimates at the regional and project levels for impacts on specific sectors, such as agriculture, energy demand for heating and cooling, water resources management and infrastructure, is growing. Based on these studies there is high confidence that there are viable adaptation options that can be implemented in some of these sectors at low cost and/or with high benefit-cost ratios. Empirical research also suggests that higher benefit-cost ratios can be achieved by implementing some adaptation measures at an early stage compared to retrofitting long-lived infrastructure at a later date. \{WGII 17.2\}

Adaptive capacity is intimately connected to social and economic development, but it is not evenly distributed across and within societies. \{WGII 7.1, 7.2, 7.4, 17.3\}

The capacity to adapt is dynamic and is influenced by a society's productive base, including natural and man-made capital assets, social networks and entitlements, human capital and institutions, governance, national income, health and technology. It is also affected by multiple climate and non-climate stresses, as well as development policy. \{WGII 17.3\}

Recent studies reaffirm the TAR finding that adaptation will be vital and beneficial. However, financial, technological, cognitive, behavioural, political, social, institutional and cultural constraints limit both the implementation and effectiveness of adaptation measures. Even societies with high adaptive capacity remain vulnerable to climate change, variability and extremes. For example, a heat wave in 2003 caused high levels of mortality in European cities (especially among the elderly), and Hurricane Katrina in 2005 caused large human and financial costs in the United States. \{WGII 7.4, 8.2, 17.4\}

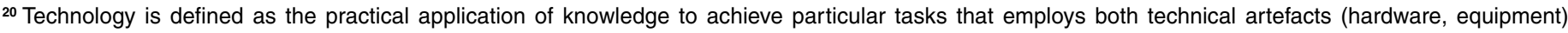
and (social) information ('software', know-how for production and use of artefacts).
} 


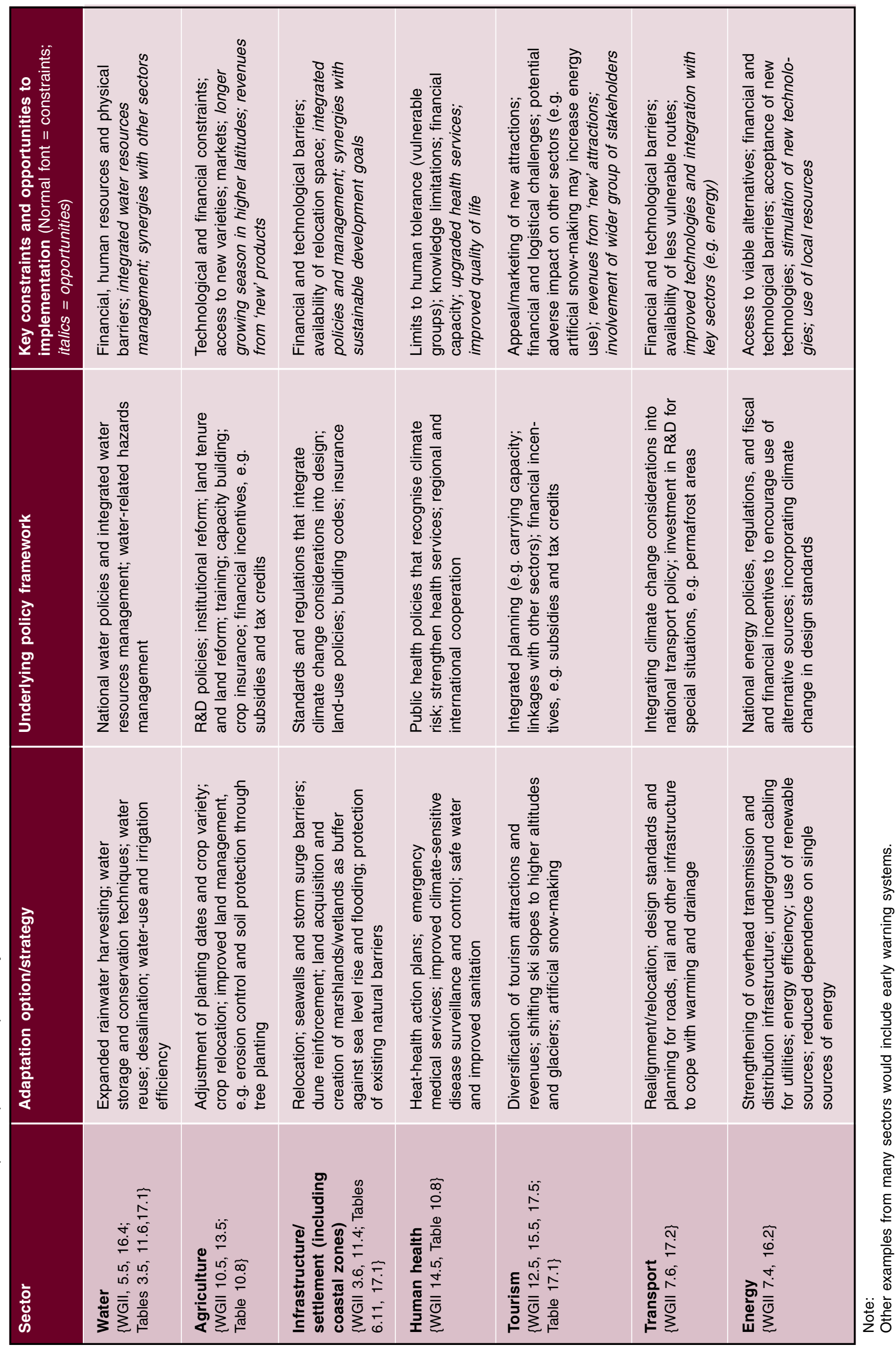




\subsection{Mitigation options}

Both bottom-up and top-down studies ${ }^{21}$ indicate that there is high agreement and much evidence of substantial economic potential ${ }^{21}$ for the mitigation of global GHG emissions over the coming decades that could offset the projected growth of global emissions or reduce emissions below current levels. \{WGIII 11.3, SPM\}

Figure 4.1 compares global economic mitigation potential in 2030 with the projected emissions increase from 2000 to 2030 . Bottom-up studies suggest that mitigation opportunities with net negative costs ${ }^{22}$ have the potential to reduce emissions by about 6 $\mathrm{GtCO}_{2}$-eq/yr in 2030. Realising these requires dealing with implementation barriers. The economic mitigation potential, which is generally greater than the market mitigation potential, can only be achieved when adequate policies are in place and barriers removed. ${ }^{21}$ \{WGIII 11.3, SPM\}
Sectoral estimates of economic mitigation potential and marginal costs derived from bottom-up studies corrected for double counting of mitigation potential are shown in Figure 4.2. While top-down and bottom-up studies are in line at the global level, there are considerable differences at the sectoral level. (WGIII 11.3, SPM)

No single technology can provide all of the mitigation potential in any sector. Table 4.2 lists selected examples of key technologies, policies, constraints and opportunities by sector. (WGIII SPM)

Future energy infrastructure investment decisions, expected to total over US $\$ 20$ trillion $^{23}$ between 2005 and 2030, will have longterm impacts on GHG emissions, because of the long lifetimes of energy plants and other infrastructure capital stock. The widespread diffusion of low-carbon technologies may take many decades, even if early investments in these technologies are made attractive. Initial estimates show that returning global energy-related $\mathrm{CO}_{2}$ emissions to 2005 levels by 2030 would require a large shift in the pattern of investment, although the net additional investment required ranges from negligible to 5 to $10 \%$. \{WGIII 4.1, 4.4, 11.6, SPM)

\section{Comparison between global economic mitigation potential and projected emissions increase in 2030}

a)
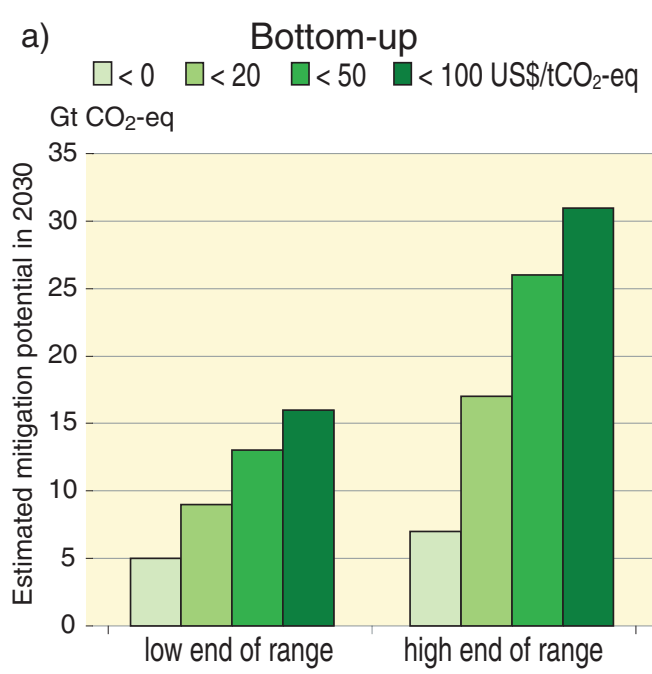

b)

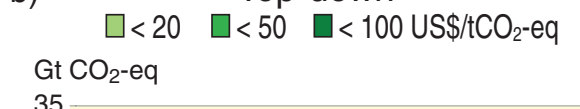

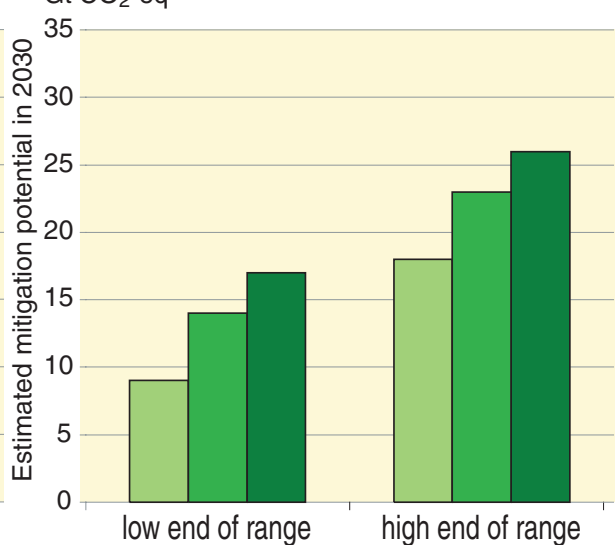

c)

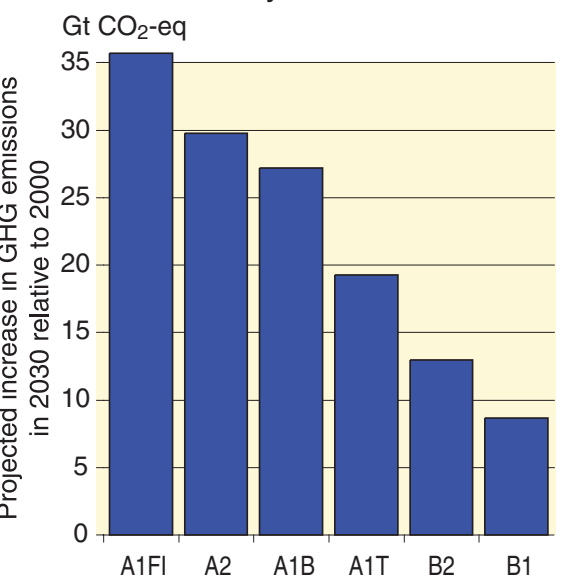

Figure 4.1. Global economic mitigation potential in 2030 estimated from bottom-up (Panel a) and top-down (Panel b) studies, compared with the projected emissions increases from SRES scenarios relative to year $2000 \mathrm{GHG}$ emissions of $40.8 \mathrm{GtCO}$-eq (Panel c). Note: GHG emissions in 2000 are exclusive of emissions of decay of above-ground biomass that remains after logging and deforestation and from peat fires and drained peat soils, to ensure consistency with the SRES emissions results. \{WGIII Figures SPM.4, SPM.5a, SPM.5b\}

\footnotetext{
${ }^{21}$ The concept of 'mitigation potential' has been developed to assess the scale of GHG reductions that could be made, relative to emission baselines, for a given level of carbon price (expressed in cost per unit of carbon dioxide equivalent emissions avoided or reduced). Mitigation potential is further differentiated in terms of 'market mitigation potential' and 'economic mitigation potential'.

Market mitigation potential is the mitigation potential based on private costs and private discount rates (reflecting the perspective of private consumers and companies), which might be expected to occur under forecast market conditions, including policies and measures currently in place, noting that barriers limit actual uptake.

Economic mitigation potential is the mitigation potential that takes into account social costs and benefits and social discount rates (reflecting the perspective of society; social discount rates are lower than those used by private investors ), assuming that market efficiency is improved by policies and measures and barriers are removed.

Mitigation potential is estimated using different types of approaches. Bottom-up studies are based on assessment of mitigation options, emphasising specific technologies and regulations. They are typically sectoral studies taking the macro-economy as unchanged. Top-down studies assess the economy-wide potential of mitigation options. They use globally consistent frameworks and aggregated information about mitigation options and capture macro-economic and market feedbacks.
}

${ }^{22}$ Net negative costs (no regrets opportunities) are defined as those options whose benefits such as reduced energy costs and reduced emissions of local/ regional pollutants equal or exceed their costs to society, excluding the benefits of avoided climate change.

${ }^{23} 20$ trillion $=20,000$ billion $=20 \times 10^{12}$ 
Economic mitigation potentials by sector in 2030 estimated from bottom-up studies

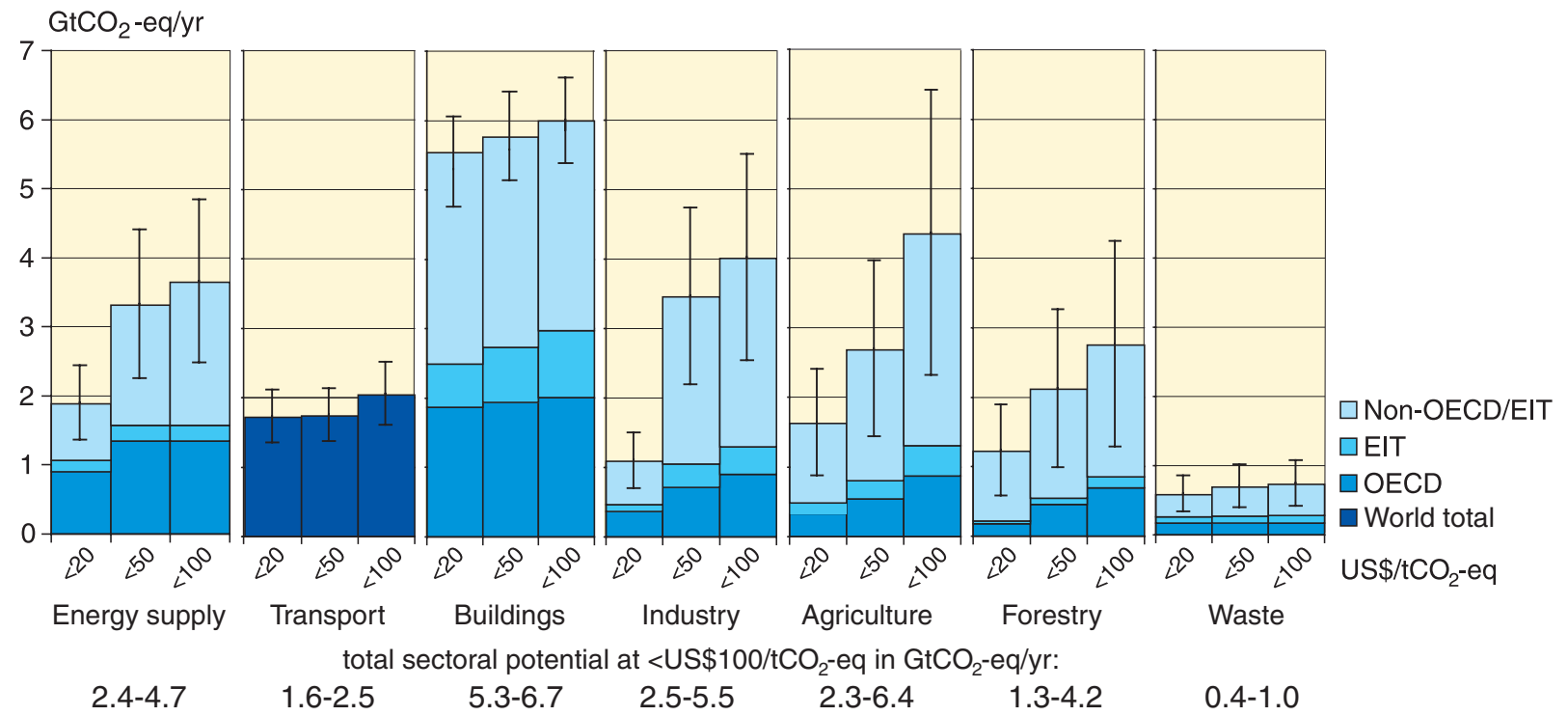

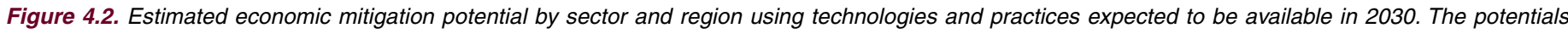
do not include non-technical options such as lifestyle changes. \{WGIII Figure SPM.6\}

Notes:

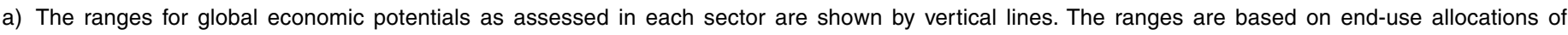
emissions, meaning that emissions of electricity use are counted towards the end-use sectors and not to the energy supply sector.

b) The estimated potentials have been constrained by the availability of studies particularly at high carbon price levels.

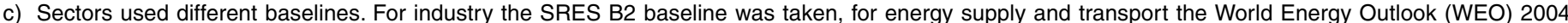
baseline was used; the building sector is based on a baseline in between SRES B2 and A1B; for waste, SRES A1B driving forces were used to construct a waste-specific baseline; agriculture and forestry used baselines that mostly used B2 driving forces.

d) Only global totals for transport are shown because international aviation is included.

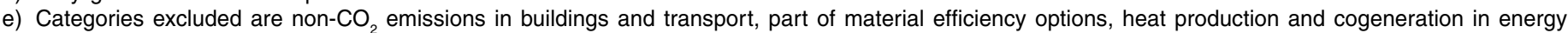
supply, heavy duty vehicles, shipping and high-occupancy passenger transport, most high-cost options for buildings, wastewater treatment, emission reduction from coal mines and gas pipelines, and fluorinated gases from energy supply and transport. The underestimation of the total economic potential from these emissions is of the order of 10 to $15 \%$.

While studies use different methodologies, there is high agreement and much evidence that in all analysed world regions near-term health co-benefits from reduced air pollution, as a result of actions to reduce GHG emissions, can be substantial and may offset a substantial fraction of mitigation costs. \{WGIII 11.8, SPM\}

Energy efficiency and utilisation of renewable energy offer synergies with sustainable development. In least developed countries, energy substitution can lower mortality and morbidity by reducing indoor air pollution, reduce the workload for women and children and decrease the unsustainable use of fuelwood and related deforestation. \{WGIII 11.8, 11.9, 12.4\}

Literature since the TAR confirms with high agreement and medium evidence that there may be effects from Annex I countries' action on the global economy and global emissions, although the scale of carbon leakage remains uncertain. \{WGIII 11.7, SPM\}

Fossil fuel exporting nations (in both Annex I and non-Annex I countries) may expect, as indicated in the TAR, lower demand and prices and lower GDP growth due to mitigation policies. The extent of this spillover depends strongly on assumptions related to policy decisions and oil market conditions. \{WGIII 11.7, SPM\}
Critical uncertainties remain in the assessment of carbon leakage. Most equilibrium modelling supports the conclusion in the TAR of economy-wide leakage from Kyoto action in the order of 5 to $20 \%$, which would be less if competitive low-emissions technologies were effectively diffused. \{WGIII 11.7, SPM \}

There is also high agreement and medium evidence that changes in lifestyle and behaviour patterns can contribute to climate change mitigation across all sectors. Management practices can also have a positive role. \{WGIII SPM\}

Examples that can have positive impacts on mitigation include changes in consumption patterns, education and training, changes in building occupant behaviour, transport demand management and management tools in industry. \{WGIII 4.1, 5.1, 6.7, 7.3, SPM\}

Policies that provide a real or implicit price of carbon could create incentives for producers and consumers to significantly invest in low-GHG products, technologies and processes. \{WGIII SPM $\}$

An effective carbon-price signal could realise significant mitigation potential in all sectors. Modelling studies show that global carbon prices rising to US $\$ 20-80 / \mathrm{tCO}_{2}$-eq by 2030 are consistent with stabilisation at around 550ppm $\mathrm{CO}_{2}$-eq by 2100 . For the same 





stabilisation level, studies since the TAR that take into account induced technological change may lower these price ranges to US\$5$65 / \mathrm{tCO}_{2}$-eq in $2030 .{ }^{24}$ \{WGIII 3.3, 11.4, 11.5, SPM \}

There is high agreement and much evidence that a wide variety of national policies and instruments are available to governments to create the incentives for mitigation action. Their applicability depends on national circumstances and an understanding of their interactions, but experience from implementation in various countries and sectors shows there are advantages and disadvantages for any given instrument. \{WGIII 13.2, SPM\}

Four main criteria are used to evaluate policies and instruments: environmental effectiveness, cost effectiveness, distributional effects including equity, and institutional feasibility. \{WGIII 13.2, SPM\}

General findings about the performance of policies are: \{WGIII 13.2, SPM\}

- Integrating climate policies in broader development policies makes implementation and overcoming barriers easier.

- Regulations and standards generally provide some certainty about emission levels. They may be preferable to other instruments when information or other barriers prevent producers and consumers from responding to price signals. However, they may not induce innovations and more advanced technologies.

- Taxes and charges can set a price for carbon, but cannot guarantee a particular level of emissions. Literature identifies taxes as an efficient way of internalising costs of GHG emissions.

- Tradable permits will establish a carbon price. The volume of allowed emissions determines their environmental effectiveness, while the allocation of permits has distributional consequences. Fluctuation in the price of carbon makes it difficult to estimate the total cost of complying with emission permits.

- Financial incentives (subsidies and tax credits) are frequently used by governments to stimulate the development and diffusion of new technologies. While economic costs are generally higher than for the instruments listed above, they are often critical to overcome barriers.

- Voluntary agreements between industry and governments are politically attractive, raise awareness among stakeholders and have played a role in the evolution of many national policies. The majority of agreements have not achieved significant emissions reductions beyond business as usual. However, some recent agreements, in a few countries, have accelerated the application of best available technology and led to measurable emission reductions.

- Information instruments (e.g. awareness campaigns) may positively affect environmental quality by promoting informed choices and possibly contributing to behavioural change, however, their impact on emissions has not been measured yet.
- Research, development and demonstration (RD\&D) can stimulate technological advances, reduce costs and enable progress toward stabilisation.

Some corporations, local and regional authorities, NGOs and civil groups are adopting a wide variety of voluntary actions. These voluntary actions may limit GHG emissions, stimulate innovative policies and encourage the deployment of new technologies. On their own, they generally have limited impact on national- or regional-level emissions. \{WGIII 13.4, SPM\}

\subsection{Relationship between adaptation and mitigation options and relationship with sustainable development}

There is growing understanding of the possibilities to choose and implement climate response options in several sectors to realise synergies and avoid conflicts with other dimensions of sustainable development. \{WGIII SPM\}

Climate change policies related to energy efficiency and renewable energy are often economically beneficial, improve energy security and reduce local pollutant emissions. Reducing both loss of natural habitat and deforestation can have significant biodiversity, soil and water conservation benefits, and can be implemented in a socially and economically sustainable manner. Forestation and bioenergy plantations can restore degraded land, manage water runoff, retain soil carbon and benefit rural economies, but could compete with food production and may be negative for biodiversity, if not properly designed. \{WGII 20.3, 20.8; WGIII 4.5, 9.7, 12.3, SPM\}

There is growing evidence that decisions about macro-economic policy, agricultural policy, multilateral development bank lending, insurance practices, electricity market reform, energy security and forest conservation, for example, which are often treated as being apart from climate policy, can significantly reduce emissions (Table 4.3). Similarly, non-climate policies can affect adaptive capacity and vulnerability. \{WGII 20.3; WGIII SPM, 12.3\}

\section{Both synergies and trade-offs exist between adaptation and mitigation options. (WGII 18.4.3; WGIII 11.9)}

Examples of synergies include properly designed biomass production, formation of protected areas, land management, energy use in buildings, and forestry, but synergies are rather limited in other sectors. Potential trade-offs include increased GHG emissions due to increased consumption of energy related to adaptive responses. (WGII 18.4.3, 18.5, 18.7, TS.5.2; WGIII 4.5, 6.9, 8.5, 9.5, SPM)

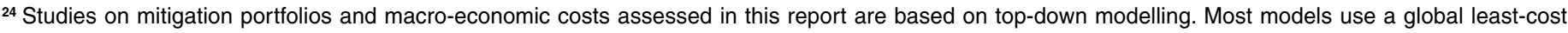

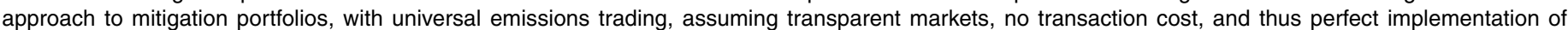

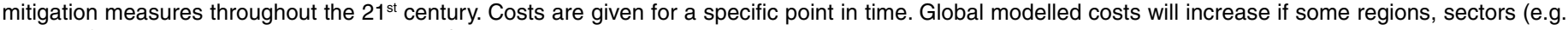

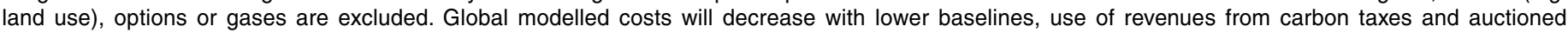

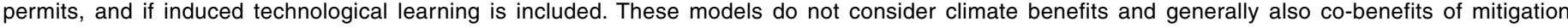

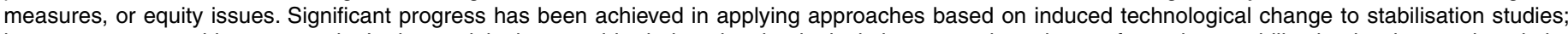

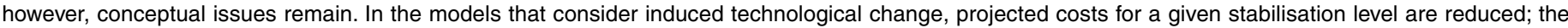
reductions are greater at lower stabilisation level.
} 
Table 4.3. Integrating climate change considerations into development policies - selected examples in the area of mitigation. $\{$ WGIII 12.2.4.6\}

\begin{tabular}{|c|c|c|}
\hline Selected sectors & Non-climate change policy instruments and actions & Potentially affects: \\
\hline Macro-economy & $\begin{array}{l}\text { Implement non-climate taxes/subsidies and/or other fiscal and } \\
\text { regulatory policies that promote sustainable development }\end{array}$ & Total global GHG emissions \\
\hline Forestry & Adoption of forest conservation and sustainable management practices & GHG emissions from deforestation \\
\hline Electricity & $\begin{array}{l}\text { Adoption of cost-effective renewables, demand-side management } \\
\text { programmes, and transmission and distribution loss reduction }\end{array}$ & Electricity sector $\mathrm{CO}_{2}$ emissions \\
\hline Petroleum imports & $\begin{array}{l}\text { Diversifying imported and domestic fuel mix and reducing } \\
\text { economy's energy intensity to improve energy security }\end{array}$ & $\begin{array}{l}\text { Emissions from crude oil and product } \\
\text { imports }\end{array}$ \\
\hline $\begin{array}{l}\text { Insurance for building, } \\
\text { transport sectors }\end{array}$ & $\begin{array}{l}\text { Differentiated premiums, liability insurance exclusions, } \\
\text { improved terms for green products }\end{array}$ & $\begin{array}{l}\text { Transport and building sector GHG } \\
\text { emissions }\end{array}$ \\
\hline International finance & Country and sector strategies and project lending that reduces emissions & Emissions from developing countries \\
\hline
\end{tabular}

\subsection{International and regional cooperation}

There is high agreement and much evidence that notable achievements of the UNFCCC and its Kyoto Protocol are the establishment of a global response to the climate change problem, stimulation of an array of national policies, the creation of an international carbon market and the establishment of new institutional mechanisms that may provide the foundation for future mitigation efforts. Progress has also been made in addressing adaptation within the UNFCCC and additional initiatives have been suggested. \{WGII 18.7; WGIII 13.3, SPM\}

The impact of the Protocol's first commitment period relative to global emissions is projected to be limited. Its economic impacts on participating Annex-B countries are projected to be smaller than presented in the TAR, which showed 0.2 to $2 \%$ lower GDP in 2012 without emissions trading and 0.1 to $1.1 \%$ lower GDP with emissions trading among Annex-B countries. To be more environmentally effective, future mitigation efforts would need to achieve deeper reductions covering a higher share of global emissions (see Topic 5). \{WGIII 1.4, 11.4, 13.3, SPM \}
The literature provides high agreement and much evidence of many options for achieving reductions of global GHG emissions at the international level through cooperation. It also suggests that successful agreements are environmentally effective, cost-effective, incorporate distributional considerations and equity, and are institutionally feasible. \{WGIII 13.3, SPM\}

Greater cooperative efforts to reduce emissions will help to reduce global costs for achieving a given level of mitigation, or will improve environmental effectiveness. Improving and expanding the scope of market mechanisms (such as emission trading, Joint Implementation and Clean Development Mechanism) could reduce overall mitigation costs. \{WGIII 13.3, SPM\}

Efforts to address climate change can include diverse elements such as emissions targets; sectoral, local, sub-national and regional actions; RD\&D programmes; adopting common policies; implementing development-oriented actions; or expanding financing instruments. These elements can be implemented in an integrated fashion, but comparing the efforts made by different countries quantitatively would be complex and resource intensive. \{ WGIII 13.3, $S P M\}$

Actions that could be taken by participating countries can be differentiated both in terms of when such action is undertaken, who participates and what the action will be. Actions can be binding or non-binding, include fixed or dynamic targets, and participation can be static or vary over time. \{WGIII 13.3, SPM\} 
The long-term perspective: scientific and socio-economic aspects relevant to adaptation and mitigation, consistent with the objectives and provisions of the Convention, and in the context of sustainable development 


\subsection{Risk management perspective}

Responding to climate change involves an iterative risk management process that includes both mitigation and adaptation, taking into account actual and avoided climate change damages, co-benefits, sustainability, equity and attitudes to risk. \{WGII 20. 9, SPM; WGIII SPM\}

Risk management techniques can explicitly accommodate sectoral, regional and temporal diversity, but their application requires information about not only impacts resulting from the most likely climate scenarios, but also impacts arising from lower-probability but higher-consequence events and the consequences of proposed policies and measures. Risk is generally understood to be the product of the likelihood of an event and its consequences. Climate change impacts depend on the characteristics of natural and human systems, their development pathways and their specific locations. \{SYR 3.3, Figure 3.6; WGII 20.2, 20.9, SPM; WGIII 3.5, 3.6, SPM\}

\subsection{Key vulnerabilities, impacts and risks - long-term perspectives}

The five 'reasons for concern' identified in the TAR are now assessed to be stronger with many risks identified with higher confidence. Some are projected to be larger or to occur at lower increases in temperature. This is due to (1) better understanding of the magnitude of impacts and risks associated with increases in global average temperature and GHG concentrations, including vulnerability to present-day climate variability, (2) more precise identification of the circumstances that make systems, sectors, groups and regions especially vulnerable and (3) growing evidence that the risk of very large impacts on multiple century time scales would continue to increase as long as GHG concentrations and temperature continue to increase. Understanding about the relationship between impacts (the basis for 'reasons for con- cern' in the TAR) and vulnerability (that includes the ability to adapt to impacts) has improved. \{WGII 4.4, 5.4, 19.ES, 19.3.7, TS.4.6; WGIII 3.5, SPM\}

The TAR concluded that vulnerability to climate change is a function of exposure, sensitivity and adaptive capacity. Adaptation can reduce sensitivity to climate change while mitigation can reduce the exposure to climate change, including its rate and extent. Both conclusions are confirmed in this assessment. \{WGII 20.2, 20.7.3\}

No single metric can adequately describe the diversity of key vulnerabilities or support their ranking. A sample of relevant impacts is provided in Figure 3.6. The estimation of key vulnerabilities in any system, and damage implied, will depend on exposure (the rate and magnitude of climate change), sensitivity, which is determined in part and where relevant by development status, and adaptive capacity. Some key vulnerabilities may be linked to thresholds; in some cases these may cause a system to shift from one state to another, whereas others have thresholds that are defined subjectively and thus depend on societal values. \{WGII 19.ES, 19.1\}

The five 'reasons for concern' that were identified in the TAR were intended to synthesise information on climate risks and key vulnerabilities and to "aid readers in making their own determination" about risk. These remain a viable framework to consider key vulnerabilities, and they have been updated in the AR4. \{TAR WGII Chapter 19; WGII SPM\}

- Risks to unique and threatened systems. There is new and stronger evidence of observed impacts of climate change on unique and vulnerable systems (such as polar and high mountain communities and ecosystems), with increasing levels of adverse impacts as temperatures increase further. An increasing risk of species extinction and coral reef damage is projected with higher confidence than in the TAR as warming proceeds. There is medium confidence that approximately 20 to $30 \%$ of plant and animal species assessed so far are likely to be at increased risk of extinction if increases in global average temperature exceed 1.5 to $2.5^{\circ} \mathrm{C}$ over $1980-1999$ levels. Confidence has increased that a 1 to $2^{\circ} \mathrm{C}$ increase in global mean temperature above 1990 levels (about 1.5 to $2.5^{\circ} \mathrm{C}$ above pre-indus-

\section{Key Vulnerabilities and Article 2 of the UNFCCC}

Article 2 of the UNFCCC states:

"The ultimate objective of this Convention and any related legal instruments that the Conference of the Parties may adopt is to achieve, in accordance with the relevant provisions of the Convention, stabilisation of greenhouse gas concentrations in the atmosphere at a level that would prevent dangerous anthropogenic interference with the climate system. Such a level should be achieved within a time frame sufficient to allow ecosystems to adapt naturally to climate change, to ensure that food production is not threatened and to enable economic development to proceed in a sustainable manner."

Determining what constitutes "dangerous anthropogenic interference with the climate system" in relation to Article 2 of the UNFCCC involves value judgements. Science can support informed decisions on this issue, including by providing criteria for judging which vulnerabilities might be labelled 'key'. \{SYR 3.3, WGII 19.ES\}

Key vulnerabilities ${ }^{25}$ may be associated with many climate-sensitive systems, including food supply, infrastructure, health, water resources, coastal systems, ecosystems, global biogeochemical cycles, ice sheets and modes of oceanic and atmospheric circulation. \{WGII 19.ES\}

More specific information is now available across the regions of the world concerning the nature of future impacts, including for some places not covered in previous assessments. \{WGII SPM\}

${ }^{25}$ Key Vulnerabilities can be identified based on a number of criteria in the literature, including magnitude, timing, persistence/reversibility, the potential for adaptation, distributional aspects, likelihood and 'importance' of the impacts. 
trial) poses significant risks to many unique and threatened systems including many biodiversity hotspots. Corals are vulnerable to thermal stress and have low adaptive capacity. Increases in sea surface temperature of about 1 to $3^{\circ} \mathrm{C}$ are projected to result in more frequent coral bleaching events and widespread mortality, unless there is thermal adaptation or acclimatisation by corals. Increasing vulnerability of Arctic indigenous communities and small island communities to warming is projected. \{SYR 3.3, 3.4, Figure 3.6, Table 3.2; WGII 4.ES, 4.4, 6.4, 14.4.6, 15.ES, 15.4, 15.6, 16.ES, 16.2.1, 16.4, Table 19.1, 19.3.7, TS.5.3, Figure TS.12, Figure TS.14\}

- Risks of extreme weather events. Responses to some recent extreme climate events reveal higher levels of vulnerability in both developing and developed countries than was assessed in the TAR. There is now higher confidence in the projected increases in droughts, heat waves and floods, as well as their adverse impacts. As summarised in Table 3.2, increases in drought, heat waves and floods are projected in many regions and would have mostly adverse impacts, including increased water stress and wild fire frequency, adverse effects on food production, adverse health effects, increased flood risk and extreme high sea level, and damage to infrastructure. \{SYR 3.2, 3.3, Table 3.2; WGI 10.3, Table SPM.2; WGII 1.3, 5.4, 7.1, 7.5, 8.2, 12.6, 19.3, Table 19.1, Table SPM.1\}

- Distribution of impacts and vulnerabilities. There are sharp differences across regions and those in the weakest economic position are often the most vulnerable to climate change and are frequently the most susceptible to climate-related damages, especially when they face multiple stresses. There is increasing evidence of greater vulnerability of specific groups such as the poor and elderly not only in developing but also in developed countries. There is greater confidence in the projected regional patterns of climate change (see Topic 3.2) and in the projections of regional impacts, enabling better identification of particularly vulnerable systems, sectors and regions (see Topic 3.3). Moreover, there is increased evidence that low-latitude and lessdeveloped areas generally face greater risk, for example in dry areas and megadeltas. New studies confirm that Africa is one of the most vulnerable continents because of the range of projected impacts, multiple stresses and low adaptive capacity. Substantial risks due to sea level rise are projected particularly for Asian megadeltas and for small island communities. \{SYR 3.2, 3.3, 5.4; WGI 11.2-11.7, SPM; WGII 3.4.3, 5.3, 5.4, Boxes 7.1 and 7.4, 8.1.1, 8.4.2, 8.6.1.3, 8.7, 9.ES, Table 10.9, 10.6, 16.3, 19.ES, 19.3, Table 19.1, 20.ES, TS.4.5, TS.5.4, Tables TS.1, TS.3, TS.4, SPM\}

- Aggregate impacts. Compared to the TAR, initial net marketbased benefits from climate change are projected to peak at a lower magnitude and therefore sooner than was assessed in the TAR. It is likely that there will be higher damages for larger magnitudes of global temperature increase than estimated in the TAR, and the net costs of impacts of increased warming are projected to increase over time. Aggregate impacts have also been quantified in other metrics (see Topic 3.3): for example, climate change over the next century is likely to adversely affect hundreds of millions of people through increased coastal flooding, reductions in water supplies, increased malnutrition and increased health impacts. \{SYR 3.3, Figure 3.6; WGII 19.3.7, 20.7.3, TS.5.3\}

- Risks of large-scale singularities. ${ }^{26}$ As discussed in Topic 3.4, during the current century, a large-scale abrupt change in the meridional overturning circulation is very unlikely. There is high confidence that global warming over many centuries would lead to a sea level rise contribution from thermal expansion alone that is projected to be much larger than observed over the $20^{\text {th }}$ century, with loss of coastal area and associated impacts. There is better understanding than in the TAR that the risk of additional contributions to sea level rise from both the Greenland and possibly Antarctic ice sheets may be larger than projected by ice sheet models and could occur on century time scales. This is because ice dynamical processes seen in recent observations but not fully included in ice sheet models assessed in the AR4 could increase the rate of ice loss. Complete deglaciation of the Greenland ice sheet would raise sea level by $7 \mathrm{~m}$ and could be irreversible. \{SYR 3.4; WGI 10.3, Box 10.1; WGII 19.3.7, SPM\}

\subsection{Adaptation and mitigation}

There is high confidence that neither adaptation nor mitigation alone can avoid all climate change impacts. Adaptation is necessary both in the short term and longer term to address impacts resulting from the warming that would occur even for the lowest stabilisation scenarios assessed. There are barriers, limits and costs that are not fully understood. Adaptation and mitigation can complement each other and together can significantly reduce the risks of climate change. \{WGII 4.ES, TS 5.1, 18.4, 18.6, 20.7, SPM; WGIII 1.2, 2.5, 3.5, 3.6\}

Adaptation will be ineffective for some cases such as natural ecosystems (e.g. loss of Arctic sea ice and marine ecosystem viability), the disappearance of mountain glaciers that play vital roles in water storage and supply, or adaptation to sea level rise of several metres ${ }^{27}$. It will be less feasible or very costly in many cases for the projected climate change beyond the next several decades (such as deltaic regions and estuaries). There is high confidence that the ability of many ecosystems to adapt naturally will be exceeded this century. In addition, multiple barriers and constraints to effective adaptation exist in human systems (see Topic 4.2). \{SYR 4.2; WGII 17.4.2, 19.2, 19.4.1\}

Unmitigated climate change would, in the long term, be likely to exceed the capacity of natural, managed and human systems to adapt. Reliance on adaptation alone could eventually lead to a magnitude of climate change to which effective adaptation is not possible, or will only be available at very high social, environmental and economic costs. \{WGII 18.1, SPM\}

\footnotetext{
${ }^{26}$ See glossary

${ }^{27}$ While it is technically possible to adapt to several metres of sea level rise, the resources required are so unevenly distributed that in reality this risk is outside the scope of adaptation. \{WGII 17.4.2, 19.4.1\}
} 
Efforts to mitigate GHG emissions to reduce the rate and magnitude of climate change need to account for inertia in the climate and socio-economic systems. \{SYR 3.2; WGI 10.3, 10.4, 10.7, SPM; WGIII 2.3.4\}

After GHG concentrations are stabilised, the rate at which the global average temperature increases is expected to slow within a few decades. Small increases in global average temperature could still be expected for several centuries. Sea level rise from thermal expansion would continue for many centuries at a rate that eventually decreases from that reached before stabilisation, due to ongoing heat uptake by oceans. \{SYR 3.2, WGI 10.3, 10.4, 10.7, SPM \}

Delayed emission reductions significantly constrain the opportunities to achieve lower stabilisation levels and increase the risk of more severe climate change impacts. Even though benefits of mitigation measures in terms of avoided climate change would take several decades to materialise, mitigation actions begun in the short term would avoid locking in both long-lived carbon intensive infrastructure and development pathways, reduce the rate of climate change and reduce the adaptation needs associated with higher levels of warming. (WGII 18.4, 20.6, 20.7, SPM; WGIII 2.3.4, 3.4, 3.5, 3.6, $S P M\}$
5.4 Emission trajectories for stabilisation

In order to stabilise the concentration of GHGs in the atmosphere, emissions would need to peak and decline thereafter. ${ }^{28}$ The lower the stabilisation level, the more quickly this peak and decline would need to occur (Figure 5.1). ${ }^{29}$ \{WGIII 3.3, 3.5, SPM\}

Advances in modelling since the TAR permit the assessment of multi-gas mitigation strategies for exploring the attainability and costs for achieving stabilisation of GHG concentrations. These scenarios explore a wider range of future scenarios, including lower levels of stabilisation, than reported in the TAR. \{WGIII 3.3, 3.5, SPM\}

Mitigation efforts over the next two to three decades will have a large impact on opportunities to achieve lower stabilisation levels (Table 5.1 and Figure 5.1). \{WGIII 3.5, SPM\}

Table 5.1 summarises the required emission levels for different groups of stabilisation concentrations and the resulting equilibrium

\section{$\mathrm{CO}_{2}$ emissions and equilibrium temperature increases for a range of stabilisation levels}
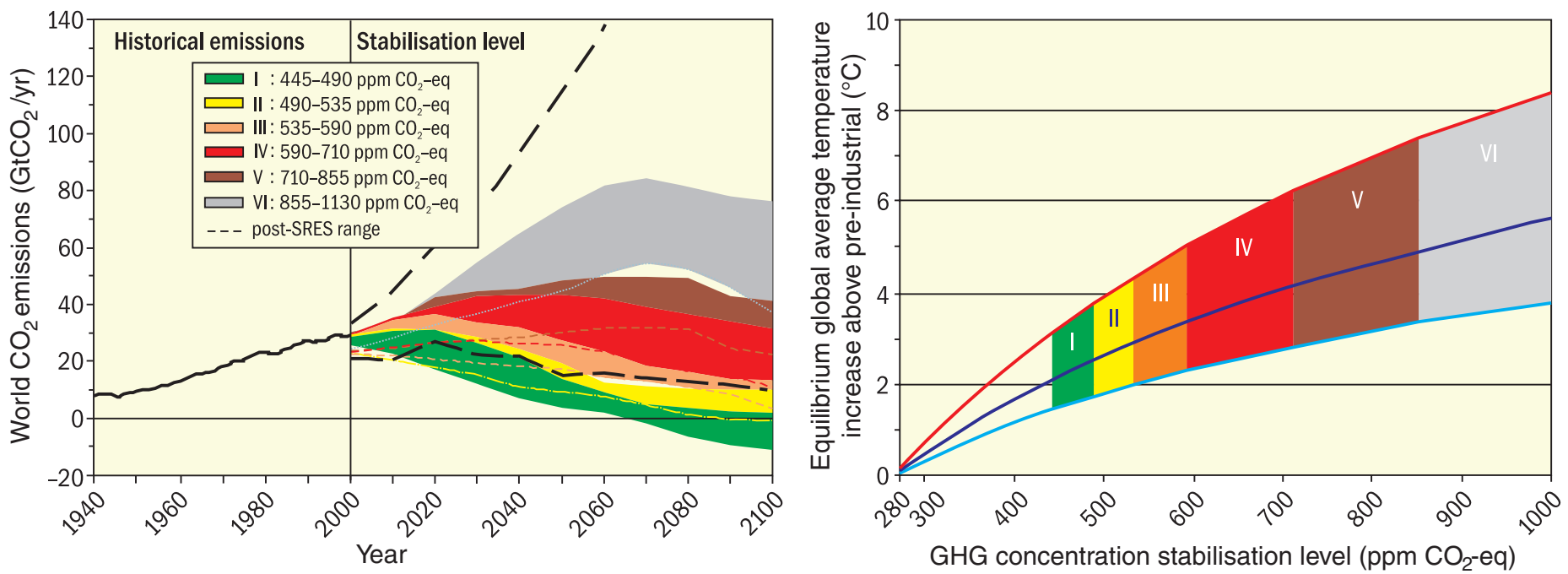

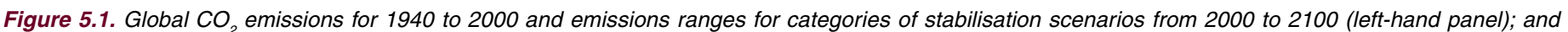

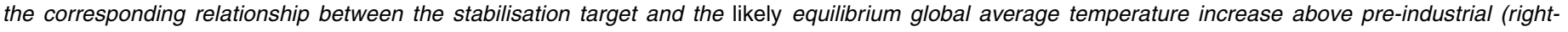

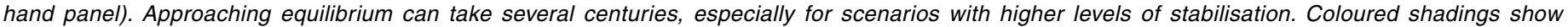

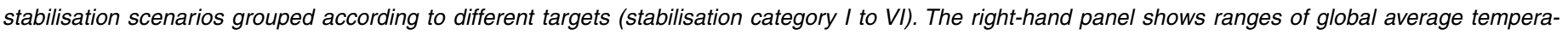

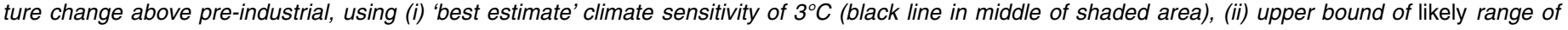

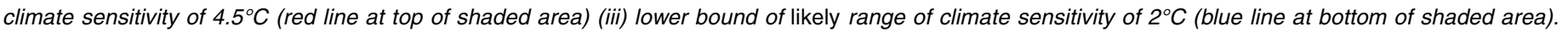

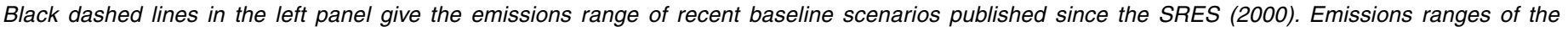

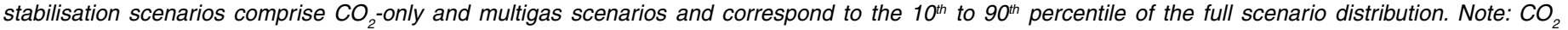

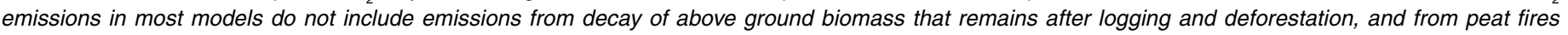
and drained peat soils. \{WGIII Figures SPM.7 and SPM.8\}

\footnotetext{
${ }^{28}$ Peaking means that the emissions need to reach a maximum before they decline later.

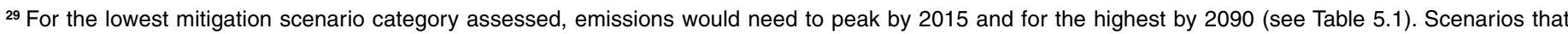
use alternative emission pathways show substantial differences on the rate of global climate change. \{WGII 19.4\}
} 
Table 5.1. Characteristics of post-TAR stabilisation scenarios and resulting long-term equilibrium global average temperature and the sea level rise component from thermal expansion only. ${ }^{a}$ \{WGI 10.7; WGIII Table TS.2, Table 3.10, Table SPM.5\}

\begin{tabular}{|c|c|c|c|c|c|c|c|}
\hline Category & $\begin{array}{l}\mathrm{CO}_{2} \\
\text { concentration } \\
\text { at stabilisation } \\
(2005=379 \\
\text { ppm })^{\mathrm{b}}\end{array}$ & $\begin{array}{l}\mathrm{CO}_{2} \text {-equivalent } \\
\text { concentration at } \\
\text { stabilisation } \\
\text { including GHGs } \\
\text { and aerosols } \\
(2005=375 \mathrm{ppm})^{\mathrm{b}}\end{array}$ & $\begin{array}{l}\text { Peaking year } \\
\text { for } \mathrm{CO}_{2} \\
\text { emissions }\end{array}$ & $\begin{array}{l}\text { Change in global } \\
\mathrm{CO}_{2} \text { emissions } \\
\text { in } 2050 \\
\text { (percent of } 2000 \\
\text { emissions) }{ }^{\mathrm{a}, \mathrm{c}}\end{array}$ & $\begin{array}{l}\text { Global average } \\
\text { temperature increase } \\
\text { above pre-industrial at } \\
\text { equilibrium, using } \\
\text { 'best estimate' climate } \\
\text { sensitivity, }\end{array}$ & $\begin{array}{l}\text { Global average sea } \\
\text { level rise above } \\
\text { pre-industrial at } \\
\text { equilibrium from } \\
\text { thermal expansion } \\
\text { only }^{\dagger}\end{array}$ & $\begin{array}{l}\text { Number of } \\
\text { assessed } \\
\text { scenarios }\end{array}$ \\
\hline & ppm & ppm & year & percent & ${ }^{\circ} \mathrm{C}$ & metres & \\
\hline I & $350-400$ & $445-490$ & $2000-2015$ & -85 to -50 & $2.0-2.4$ & $0.4-1.4$ & 6 \\
\hline II & $400-440$ & $490-535$ & $2000-2020$ & -60 to -30 & $2.4-2.8$ & $0.5-1.7$ & 18 \\
\hline III & $440-485$ & $535-590$ & $2010-2030$ & -30 to +5 & $2.8-3.2$ & $0.6-1.9$ & 21 \\
\hline IV & $485-570$ & $590-710$ & $2020-2060$ & +10 to +60 & $3.2-4.0$ & $0.6-2.4$ & 118 \\
\hline V & $570-660$ & $710-855$ & $2050-2080$ & +25 to +85 & $4.0-4.9$ & $0.8-2.9$ & 9 \\
\hline VI & $660-790$ & $855-1130$ & $2060-2090$ & +90 to +140 & $4.9-6.1$ & $1.0-3.7$ & 5 \\
\hline
\end{tabular}

Notes:

a) The emission reductions to meet a particular stabilisation level reported in the mitigation studies assessed here might be underestimated due to missing carbon cycle feedbacks (see also Topic 2.3).

b) Atmospheric $\mathrm{CO}_{2}$ concentrations were $379 \mathrm{ppm}$ in 2005 . The best estimate of total $\mathrm{CO}_{2}$-eq concentration in 2005 for all long-lived GHGs is about $455 \mathrm{ppm}$, while the corresponding value including the net effect of all anthropogenic forcing agents is $375 \mathrm{ppm}^{\mathrm{CO}}$-eq

c) Ranges correspond to the $15^{\text {th }}$ to $85^{\text {th }}$ percentile of the post-TAR scenario distribution. $\mathrm{CO}_{2}$ emissions are shown so multi-gas scenarios can be compared with $\mathrm{CO}_{2}$-only scenarios (see Figure 2.1).

d) The best estimate of climate sensitivity is $3^{\circ} \mathrm{C}$

e) Note that global average temperature at equilibrium is different from expected global average temperature at the time of stabilisation of GHG concentrations due to the inertia of the climate system. For the majority of scenarios assessed, stabilisation of GHG concentrations occurs between 2100 and 2150 (see also Footnote 30).

f) Equilibrium sea level rise is for the contribution from ocean thermal expansion only and does not reach equilibrium for at least many centuries. These values have been estimated using relatively simple climate models (one low-resolution AOGCM and several EMICs based on the best estimate of $3^{\circ} \mathrm{C}$ climate sensitivity) and do not include contributions from melting ice sheets, glaciers and ice caps. Long-term thermal expansion is projected to result in 0.2 to $0.6 \mathrm{~m}$ per degree Celsius of global average warming above pre-industrial. (AOGCM refers to Atmosphere-Ocean General Circulation Model and EMICs to Earth System Models of Intermediate Complexity.)

global average temperature increases, using the 'best estimate' of climate sensitivity (see Figure 5.1 for the likely range of uncertainty). Stabilisation at lower concentration and related equilibrium temperature levels advances the date when emissions need to peak and requires greater emissions reductions by $2050 .{ }^{30}$ Climate sensitivity is a key uncertainty for mitigation scenarios that aim to meet specific temperature levels. The timing and level of mitigation to reach a given temperature stabilisation level is earlier and more stringent if climate sensitivity is high than if it is low. \{WGIII 3.3, 3.4, 3.5, 3.6, SPM\}

Sea level rise under warming is inevitable. Thermal expansion would continue for many centuries after GHG concentrations have stabilised, for any of the stabilisation levels assessed, causing an eventual sea level rise much larger than projected for the $21^{\text {st }}$ century (Table 5.1). If GHG and aerosol concentrations had been stabilised at year 2000 levels, thermal expansion alone would be expected to lead to further sea level rise of 0.3 to $0.8 \mathrm{~m}$. The eventual contributions from Greenland ice sheet loss could be several metres, and larger than from thermal expansion, should warming in excess of 1.9 to $4.6^{\circ} \mathrm{C}$ above pre-industrial be sustained over many centuries. These long-term consequences would have major impli- cations for world coastlines. The long time scale of thermal expansion and ice sheet response to warming imply that mitigation strategies that seek to stabilise GHG concentrations (or radiative forcing) at or above present levels do not stabilise sea level for many centuries. \{WG1 10.7\}

Feedbacks between the carbon cycle and climate change affect the required mitigation and adaptation response to climate change. Climate-carbon cycle coupling is expected to increase the fraction of anthropogenic emissions that remains in the atmosphere as the climate system warms (see Topics 2.3 and 3.2.1), but mitigation studies have not yet incorporated the full range of these feedbacks. As a consequence, the emission reductions to meet a particular stabilisation level reported in the mitigation studies assessed in Table 5.1 might be underestimated. Based on current understanding of climate-carbon cycle feedbacks, model studies suggest that stabilising $\mathrm{CO}_{2}$ concentrations at, for example, $450 \mathrm{ppm}^{31}$ could require cumulative emissions over the $21^{\text {st }}$ century to be less than 1800 [1370 to 2200] $\mathrm{GtCO}_{2}$, which is about $27 \%$ less than the 2460 [2310 to 2600] $\mathrm{GtCO}_{2}$ determined without consideration of carbon cycle feedbacks. \{SYR 2.3, 3.2.1; WGI 7.3, 10.4, SPM\}

\footnotetext{
${ }^{30}$ Estimates for the evolution of temperature over the course of this century are not available in the AR4 for the stabilisation scenarios. For most stabilisation levels global average temperature is approaching the equilibrium level over a few centuries. For the much lower stabilisation scenarios (category I and II, Figure 5.1), the equilibrium temperature may be reached earlier.

${ }^{31}$ To stabilise at $1000 \mathrm{ppm} \mathrm{CO}_{2}$, this feedback could require that cumulative emissions be reduced from a model average of approximately 5190 [4910 to 5460] $\mathrm{GtCO}_{2}$ to approximately 4030 [3590 to 4580] $\mathrm{GtCO}_{2}$. $\{$ WGI 7.3, 10.4, SPM $\}$
} 


\subsection{Technology flows and development}

There is high agreement and much evidence that all stabilisation levels assessed can be achieved by deployment of a portfolio of technologies that are either currently available or expected to be commercialised in coming decades, assuming appropriate and effective incentives are in place for development, acquisition, deployment and diffusion of technologies and addressing related barriers. \{WGIII SPM\}

Worldwide deployment of low-GHG emission technologies as well as technology improvements through public and private RD\&D would be required for achieving stabilisation targets as well as cost reduction. ${ }^{32}$ Figure 5.2 gives illustrative examples of the contribution of the portfolio of mitigation options. The contribution of different technologies varies over time and region and depends on the baseline development path, available technologies and relative costs, and the analysed stabilisation levels. Stabilisation at the lower of the assessed levels (490 to 540ppm $\mathrm{CO}_{2}$-eq) requires early investments and substantially more rapid diffusion and commercialisation of advanced low-emissions technologies over the next decades
(2000-2030) and higher contributions across abatement options in the long term (2000-2100). This requires that barriers to development, acquisition, deployment and diffusion of technologies are effectively addressed with appropriate incentives. [WGIII 2.7, 3.3, 3.4, 3.6, 4.3, 4.4, 4.6, SPM\}

Without sustained investment flows and effective technology transfer, it may be difficult to achieve emission reduction at a significant scale. Mobilising financing of incremental costs of lowcarbon technologies is important. \{WGIII 13.3, SPM\}

There are large uncertainties concerning the future contribution of different technologies. However, all assessed stabilisation scenarios concur that 60 to $80 \%$ of the reductions over the course of the century would come from energy supply and use and industrial processes. Including non- $\mathrm{CO}_{2}$ and $\mathrm{CO}_{2}$ land-use and forestry mitigation options provides greater flexibility and cost-effectiveness. Energy efficiency plays a key role across many scenarios for most regions and time scales. For lower stabilisation levels, scenarios put more emphasis on the use of low-carbon energy sources, such as renewable energy, nuclear power and the use of $\mathrm{CO}_{2}$ capture and storage (CCS). In these scenarios, improvements of carbon intensity of energy supply and the whole economy needs to be much faster than in the past (Figure 5.2). \{WGIII 3.3, 3.4, TS.3, SPM\}

Illustrative mitigation portfolios for achieving stabilisation of GHG concentrations

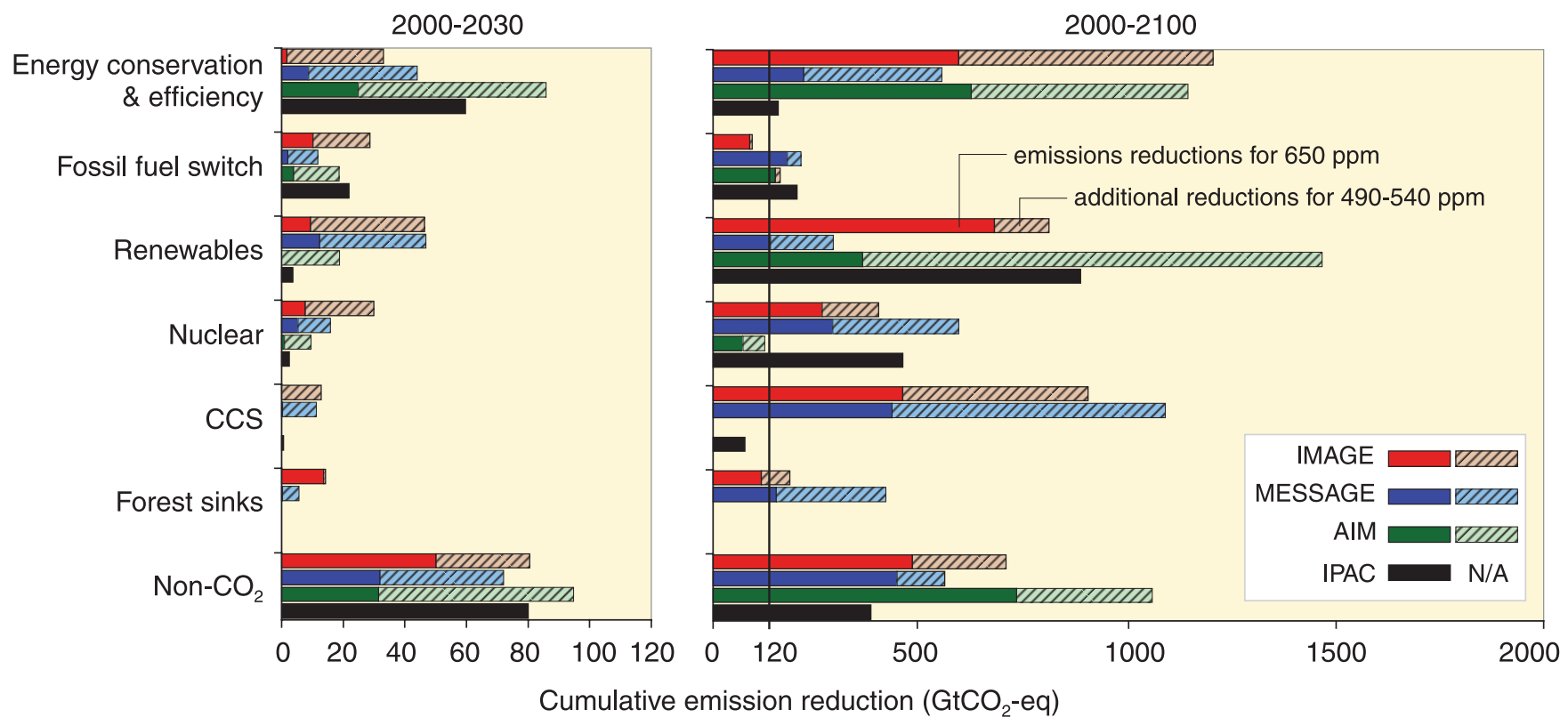

Figure 5.2 Cumulative emissions reductions for alternative mitigation measures for 2000-2030 (left-hand panel) and for 2000-2100 (right-hand panel). The figure shows illustrative scenarios from four models (AIM, IMAGE, IPAC and MESSAGE) aiming at the stabilisation at low (490 to 540ppm CO-eq) and intermediate levels (650ppm CO${ }_{2}^{-e q)}$ respectively. Dark bars denote reductions for a target of 650ppm $\mathrm{CO}_{2}$-eq and light bars denote the additional reductions to achieve 490 to $540 \mathrm{ppm} \mathrm{CO}$-eq. Note that some models do not consider mitigation through forest sink enhancement (AIM and IPAC) or CCS (AIM) and that the share of low-carbon energy options in total energy supply is also determined by inclusion of these options in the baseline. CCS includes $\mathrm{CO}_{2}$ capture and storage from biomass. Forest sinks include reducing emissions from deforestation. The figure shows emissions reductions from baseline scenarios with cumulative emissions between 6000 to $7000 \mathrm{GtCO}_{2}-e q$ (2000-2100). \{WGIII Figure SPM.9\}

\footnotetext{
${ }^{32}$ By comparison, government funding in real absolute terms for most energy research programmes has been flat or declining for nearly two decades (even after the UNFCCC came into force) and is now about half of the 1980 level. \{WGIII 2.7, 3.4, 4.5, 11.5, 13.2\}
} 


\subsection{Costs of mitigation and long-term stabilisation targets}

The macro-economic costs of mitigation generally rise with the stringency of the stabilisation target and are relatively higher when derived from baseline scenarios characterised by high emission levels. \{WGIII SPM\}

There is high agreement and medium evidence that in 2050 global average macro-economic costs for multi-gas mitigation towards stabilisation between 710 and $445 \mathrm{ppm} \mathrm{CO}_{2}$-eq are between a $1 \%$ gain to a $5.5 \%$ decrease of global GDP (Table 5.2). This corresponds to slowing average annual global GDP growth by less than 0.12 percentage points. Estimated GDP losses by 2030 are on average lower and show a smaller spread compared to 2050 (Table 5.2). For specific countries and sectors, costs vary considerably from the global average. ${ }^{33}$ \{WGIII 3.3, 13.3, SPM\}

\subsection{Costs, beneffits and avoided climate impacts at global and regional levels}

Impacts of climate change will vary regionally. Aggregated and discounted to the present, they are very likely to impose net annual costs, which will increase over time as global temperatures increase. \{WGII SPM\}

For increases in global average temperature of less than 1 to $3^{\circ} \mathrm{C}$ above 1980-1999 levels, some impacts are projected to produce market benefits in some places and sectors while, at the same time, imposing costs in other places and sectors. Global mean losses could be 1 to $5 \%$ of GDP for $4{ }^{\circ} \mathrm{C}$ of warming, but regional losses could be substantially higher. \{WGII 9.ES, 10.6, 15.ES, 20.6, SPM\}

Peer-reviewed estimates of the social cost of carbon (net economic costs of damages from climate change aggregated across the globe and discounted to the present) for 2005 have an average value of US\$12 per tonne of $\mathrm{CO}_{2}$, but the range from 100 estimates is large ( $-\$ 3$ to $\$ 95 / \mathrm{tCO}_{2}$ ). The range of published evidence indicates that the net damage costs of climate change are projected to be significant and to increase over time. \{WGII 20.6, SPM\}

It is very likely that globally aggregated figures underestimate the damage costs because they cannot include many non-quantifiable impacts. It is virtually certain that aggregate estimates of costs mask significant differences in impacts across sectors, regions, countries and populations. In some locations and amongst some groups of people with high exposure, high sensitivity and/or low adaptive capacity, net costs will be significantly larger than the global average. \{WGII 7.4, 20.ES, 20.6, 20.ES, SPM\}

Limited and early analytical results from integrated analyses of the global costs and benefits of mitigation indicate that these are broadly comparable in magnitude, but do not as yet permit an unambiguous determination of an emissions pathway or stabilisation level where benefits exceed costs. \{WGIII SPM\}

Comparing the costs of mitigation with avoided damages would require the reconciliation of welfare impacts on people living in different places and at different points in time into a global aggregate measure of well-being. \{WGII 18.ES\}

Choices about the scale and timing of GHG mitigation involve balancing the economic costs of more rapid emission reductions now against the corresponding medium-term and long-term climate risks of delay. \{WGIII SPM\}

Many impacts can be avoided, reduced or delayed by mitigation. \{WGII SPM\}

Although the small number of impact assessments that evaluate stabilisation scenarios do not take full account of uncertainties in projected climate under stabilisation, they nevertheless provide indications of damages avoided and risks reduced for different

Table 5.2. Estimated global macro-economic costs in 2030 and 2050. Costs are relative to the baseline for least-cost trajectories towards different long-term stabilisation levels. \{WGIII 3.3, 13.3, Tables SPM.4 and SPM.6\}

\begin{tabular}{|c|c|c|c|c|c|c|}
\hline \multirow[t]{2}{*}{$\begin{array}{l}\text { Stabilisation levels } \\
\text { (ppm } \mathrm{CO}_{2} \text {-eq) }\end{array}$} & \multicolumn{2}{|c|}{ Median GDP reduction ${ }^{\mathrm{a}}(\%)$} & \multicolumn{2}{|c|}{ Range of GDP reduction ${ }^{\mathrm{b}}(\%)$} & \multicolumn{2}{|c|}{$\begin{array}{l}\text { Reduction of average annual GDP } \\
\text { growth rates (percentage points) }\end{array}$} \\
\hline & 2030 & 2050 & 2030 & 2050 & 2030 & 2050 \\
\hline $445-535^{d}$ & \multicolumn{2}{|c|}{ Not available } & $<3$ & $<5.5$ & $<0.12$ & $<0.12$ \\
\hline $535-590$ & 0.6 & 1.3 & 0.2 to 2.5 & slightly negative to 4 & $<0.1$ & $<0.1$ \\
\hline $590-710$ & 0.2 & 0.5 & -0.6 to 1.2 & -1 to 2 & $<0.06$ & $<0.05$ \\
\hline
\end{tabular}

Notes:

Values given in this table correspond to the full literature across all baselines and mitigation scenarios that provide GDP numbers.

a) Global GDP based on market exchange rates.

b) The $10^{\text {th }}$ and $90^{\text {th }}$ percentile range of the analysed data are given where applicable. Negative values indicate GDP gain. The first row (445-535ppm $\mathrm{CO}_{2}$-eq) gives the upper bound estimate of the literature only.

c) The calculation of the reduction of the annual growth rate is based on the average reduction during the assessed period that would result in the indicated GDP decrease by 2030 and 2050 respectively.

d) The number of studies is relatively small and they generally use low baselines. High emissions baselines generally lead to higher costs.

e) The values correspond to the highest estimate for GDP reduction shown in column three.

\footnotetext{
${ }^{33}$ See Footnote 24 for further details on cost estimates and model assumptions.
} 
amounts of emissions reduction. The rate and magnitude of future human-induced climate change and its associated impacts are determined by human choices defining alternative socio-economic futures and mitigation actions that influence emission pathways. Figure 3.2 demonstrates that alternative SRES emission pathways could lead to substantial differences in climate change throughout the $21^{\text {st }}$ century. Some of the impacts at the high temperature end of Figure 3.6 could be avoided by socio-economic development pathways that limit emissions and associated climate change towards the lower end of the ranges illustrated in Figure 3.6. \{SYR 3.2, 3.3; WGIII 3.5, 3.6, SPM\}

Figure 3.6 illustrates how reduced warming could reduce the risk of, for example, affecting a significant number of ecosystems, the risk of extinctions, and the likelihood that cereal productivity in some regions would tend to fall. \{SYR 3.3, Figure 3.6; WGII 4.4, 5.4, Table 20.6\}

\subsection{Broader environmental and sustainability issues}

Sustainable development can reduce vulnerability to climate
change, and climate change could impede nations' abilities
to achieve sustainable development pathways. \{WGII SPM\}

It is very likely that climate change can slow the pace of progress toward sustainable development either directly through increased exposure to adverse impacts or indirectly through erosion of the capacity to adapt. Over the next half-century, climate change could impede achievement of the Millennium Development Goals. \{WGII $S P M\}$

Climate change will interact at all scales with other trends in global environmental and natural resource concerns, including water, soil and air pollution, health hazards, disaster risk, and deforestation. Their combined impacts may be compounded in future in the absence of integrated mitigation and adaptation measures. \{WGII 20.3, 20.7, 20.8, SPM\}

Making development more sustainable can enhance mitigative and adaptive capacities, reduce emissions, and reduce vulnerability, but there may be barriers to implementation. \{WGII 20.8; WGIII 12.2, SPM\}

Both adaptive and mitigative capacities can be enhanced through sustainable development. Sustainable development can, thereby, reduce vulnerability to climate change by reducing sensitivities (through adaptation) and/or exposure (through mitigation). At present, however, few plans for promoting sustainability have explicitly included either adapting to climate change impacts, or promoting adaptive capacity. Similarly, changing development paths can make a major contribution to mitigation but may require resources to overcome multiple barriers. [WGII 20.3, 20.5, SPM; WGIII $2.1,2.5,12.1, S P M\}$ 
Robust findings, key uncertainties 


\section{Robust findings, key uncertainties}

As in the TAR, a robust finding for climate change is defined as one that holds under a variety of approaches, methods, models and assumptions, and is expected to be relatively unaffected by uncertainties. Key uncertainties are those that, if reduced, could lead to new robust findings. \{TAR SYR Q.9\}

Robust findings do not encompass all key findings of the AR4. Some key findings may be policy-relevant even though they are associated with large uncertainties. \{WGII 20.9\}

The robust findings and key uncertainties listed below do not represent an exhaustive list.

\subsection{Observed changes in climate and their effects, and their causes}

\section{Robust findings}

Warming of the climate system is unequivocal, as is now evident from observations of increases in global average air and ocean temperatures, widespread melting of snow and ice and rising global average sea level. \{WGI 3.9, SPM \}

Many natural systems, on all continents and in some oceans, are being affected by regional climate changes. Observed changes in many physical and biological systems are consistent with warming. As a result of the uptake of anthropogenic $\mathrm{CO}_{2}$ since 1750, the acidity of the surface ocean has increased. \{WGI 5.4, WGII 1.3\}

Global total annual anthropogenic GHG emissions, weighted by their 100-year GWPs, have grown by $70 \%$ between 1970 and 2004. As a result of anthropogenic emissions, atmospheric concentrations of $\mathrm{N}_{2} \mathrm{O}$ now far exceed pre-industrial values spanning many thousands of years, and those of $\mathrm{CH}_{4}$ and $\mathrm{CO}_{2}$ now far exceed the natural range over the last 650,000 years. \{WGI SPM; WGIII 1.3\}

Most of the global average warming over the past 50 years is very likely due to anthropogenic GHG increases and it is likely that there is a discernible human-induced warming averaged over each continent (except Antarctica). \{WGI 9.4, SPM\}

Anthropogenic warming over the last three decades has likely had a discernible influence at the global scale on observed changes in many physical and biological systems. \{WGII 1.4, SPM \}

\section{Key uncertainties}

Climate data coverage remains limited in some regions and there is a notable lack of geographic balance in data and literature on observed changes in natural and managed systems, with marked scarcity in developing countries. \{WGI SPM; WGII 1.3, SPM\}

Analysing and monitoring changes in extreme events, including drought, tropical cyclones, extreme temperatures and the frequency and intensity of precipitation, is more difficult than for climatic averages as longer data time-series of higher spatial and temporal resolutions are required. \{WGI 3.8, SPM \}

Effects of climate changes on human and some natural systems are difficult to detect due to adaptation and non-climatic drivers. \{WGII 1.3\}
Difficulties remain in reliably simulating and attributing observed temperature changes to natural or human causes at smaller than continental scales. At these smaller scales, factors such as landuse change and pollution also complicate the detection of anthropogenic warming influence on physical and biological systems. \{WGI 8.3, 9.4, SPM; WGII 1.4, SPM\}

The magnitude of $\mathrm{CO}_{2}$ emissions from land-use change and $\mathrm{CH}_{4}$ emissions from individual sources remain as key uncertainties. \{WGI 2.3, 7.3, 7.4; WGIII 1.3, TS.14\}

\subsection{Drivers and projections of future climate changes and their impacts}

\section{Robust findings}

With current climate change mitigation policies and related sustainable development practices, global GHG emissions will continue to grow over the next few decades. \{WGIII 3.2, SPM\}

For the next two decades a warming of about $0.2^{\circ} \mathrm{C}$ per decade is projected for a range of SRES emissions scenarios. [WGI 10.3, 10.7, SPM\}

Continued GHG emissions at or above current rates would cause further warming and induce many changes in the global climate system during the $21^{\text {st }}$ century that would very likely be larger than those observed during the $20^{\text {th }}$ century. \{WGI 10.3, 11.1, SPM\}

The pattern of future warming where land warms more than the adjacent oceans and more in northern high latitudes is seen in all scenarios. \{WGI 10.3, 11.1, SPM\}

Warming tends to reduce terrestrial ecosystem and ocean uptake of atmospheric $\mathrm{CO}_{2}$, increasing the fraction of anthropogenic emissions that remains in the atmosphere. \{WGI 7.3, 10.4, 10.5, SPM \}

Anthropogenic warming and sea level rise would continue for centuries even if GHG emissions were to be reduced sufficiently for GHG concentrations to stabilise, due to the time scales associated with climate processes and feedbacks. \{WGI 10.7, SPM\}

Equilibrium climate sensitivity is very unlikely to be less than $1.5^{\circ} \mathrm{C}$. \{WGI 8.6, 9.6, Box 10.2, SPM\}

Some systems, sectors and regions are likely to be especially affected by climate change. The systems and sectors are some ecosystems (tundra, boreal forest, mountain, mediterranean-type, mangroves, salt marshes, coral reefs and the sea-ice biome), low-lying coasts, water resources in some dry regions at mid-latitudes and in the dry topics and in areas dependent on snow and ice melt, agriculture in low-latitude regions, and human health in areas with low adaptive capacity. The regions are the Arctic, Africa, small islands and Asian and African megadeltas. Within other regions, even those with high incomes, some people, areas and activities can be particularly at risk. \{WGII TS.4.5\}

Impacts are very likely to increase due to increased frequencies and intensities of some extreme weather events. Recent events have demonstrated the vulnerability of some sectors and regions, including in developed countries, to heat waves, tropical cyclones, floods and drought, providing stronger reasons for concern as compared to the findings of the TAR. \{WGII Table SPM.2, 19.3\} 


\section{Key uncertainties}

Uncertainty in the equilibrium climate sensitivity creates uncertainty in the expected warming for a given $\mathrm{CO}_{2}$-eq stabilisation scenario. Uncertainty in the carbon cycle feedback creates uncertainty in the emissions trajectory required to achieve a particular stabilisation level. \{WGI 7.3, 10.4, 10.5, SPM\}

Models differ considerably in their estimates of the strength of different feedbacks in the climate system, particularly cloud feedbacks, oceanic heat uptake and carbon cycle feedbacks, although progress has been made in these areas. Also, the confidence in projections is higher for some variables (e.g. temperature) than for others (e.g. precipitation), and it is higher for larger spatial scales and longer time averaging periods. \{WGI 7.3, 8.1-8.7, 9.6, 10.2, 10.7, SPM; WGII 4.4\}

Aerosol impacts on the magnitude of the temperature response, on clouds and on precipitation remain uncertain. \{WGI 2.9, 7.5, 9.2, 9.4, 9.5\}

Future changes in the Greenland and Antarctic ice sheet mass, particularly due to changes in ice flow, are a major source of uncertainty that could increase sea level rise projections. The uncertainty in the penetration of the heat into the oceans also contributes to the future sea level rise uncertainty. \{WGI 4.6, 6.4, 10.3, 10.7, SPM\}

Large-scale ocean circulation changes beyond the $21^{\text {st }}$ century cannot be reliably assessed because of uncertainties in the meltwater supply from the Greenland ice sheet and model response to the warming. \{WGI 6.4, 8.7, 10.3 \}

Projections of climate change and its impacts beyond about 2050 are strongly scenario- and model-dependent, and improved projections would require improved understanding of sources of uncertainty and enhancements in systematic observation networks. \{WGII TS.6\}

Impacts research is hampered by uncertainties surrounding regional projections of climate change, particularly precipitation. \{WGII TS.6\}

Understanding of low-probability/high-impact events and the cumulative impacts of sequences of smaller events, which is required for risk-based approaches to decision-making, is generally limited. \{WGII 19.4, 20.2, 20.4, 20.9, TS.6\}

\subsection{Responses to climate change}

\section{Robust findings}

Some planned adaptation (of human activities) is occurring now; more extensive adaptation is required to reduce vulnerability to climate change. \{WGII 17.ES, 20.5, Table 20.6, SPM\}

Unmitigated climate change would, in the long term, be likely to exceed the capacity of natural, managed and human systems to adapt. \{WGII 20.7, SPM\}
A wide range of mitigation options is currently available or projected to be available by 2030 in all sectors. The economic mitigation potential, at costs that range from net negative up to US\$100/ $\mathrm{tCO}_{2}$-equivalent, is sufficient to offset the projected growth of global emissions or to reduce emissions to below current levels in 2030. \{WGIII 11.3, SPM\}

Many impacts can be reduced, delayed or avoided by mitigation. Mitigation efforts and investments over the next two to three decades will have a large impact on opportunities to achieve lower stabilisation levels. Delayed emissions reductions significantly constrain the opportunities to achieve lower stabilisation levels and increase the risk of more severe climate change impacts. \{WGII SPM, WGIII SPM\}

The range of stabilisation levels for GHG concentrations that have been assessed can be achieved by deployment of a portfolio of technologies that are currently available and those that are expected to be commercialised in coming decades, provided that appropriate and effective incentives are in place and barriers are removed. In addition, further RD\&D would be required to improve the technical performance, reduce the costs and achieve social acceptability of new technologies. The lower the stabilisation levels, the greater the need for investment in new technologies during the next few decades. \{WGIII 3.3, 3.4\}

Making development more sustainable by changing development paths can make a major contribution to climate change mitigation and adaptation and to reducing vulnerability. (WGII 18.7, 20.3, SPM; WGIII 13.2, SPM\}

Decisions about macro-economic and other policies that seem unrelated to climate change can significantly affect emissions. \{WGIII $12.2\}$

\section{Key uncertainties}

Understanding of how development planners incorporate information about climate variability and change into their decisions is limited. This limits the integrated assessment of vulnerability. \{WGII 18.8, 20.9\}

The evolution and utilisation of adaptive and mitigative capacity depend on underlying socio-economic development pathways. \{WGII 17.3, 17.4, 18.6, 19.4, 20.9\}

Barriers, limits and costs of adaptation are not fully understood, partly because effective adaptation measures are highly dependent on specific geographical and climate risk factors as well as institutional, political and financial constraints. \{WGII SPM\}

Estimates of mitigation costs and potentials depend on assumptions about future socio-economic growth, technological change and consumption patterns. Uncertainty arises in particular from assumptions regarding the drivers of technology diffusion and the potential of long-term technology performance and cost improvements. Also little is known about the effects of changes in behaviour and lifestyles. \{WGIII 3.3, 3.4, 11.3\}

The effects of non-climate policies on emissions are poorly quantified. \{WGIII 12.2\} 



\section{Annex I}

\section{User guide and access to more detailed information}

As defined in the IPCC Procedures, the Synthesis Report (SYR) synthesises and integrates material contained within IPCC Assessment Reports and Special Reports. The scope of the SYR of the Fourth Assessment Report includes material contained in the three Working Group contributions to the AR4, and it draws on information contained in other IPCC Reports as required. The SYR is based exclusively on assessments by the IPCC Working Groups, it does not refer to or assess the primary scientific literature itself.

The SYR is largely self-contained but provides only a very condensed summary of the much richer information contained in the underlying Working Group reports. Users may wish to access relevant material at the required level of detail in the following manner:

- The Summary for Policymakers (SPM) of the SYR provides the most condensed summary of our current understanding of scientific, technical and socio-economic aspects of climate change. All references in curly brackets in this Summary for Policymakers refer to numbered sections of this SYR.

- The Introduction and six Topics of this SYR provide more detailed and more comprehensive information than the SYR SPM. References in curly backets in the Introduction and six Topics of this SYR point to chapter sections, Summaries for Policymakers and Technical Summaries of the three underlying Working Group reports of the AR4, and in some instances to other topic sections of the SYR itself. References to the IPCC Third Assessment Report in 2001 (TAR) are identified by adding "TAR" in front of the cited report.

- Users who wish to gain a better understanding of scientific details or access the primary scientific literature on which the SYR is based, should refer to chapter sections of the underlying Working Group reports that are cited in the longer report of the SYR. The individual chapters of the Working Group reports provide comprehensive references to the primary scientific literature on which IPCC assessments are based, and also offer the most detailed region- and sector-specific information.

A comprehensive glossary, list of acronyms, abbreviations and scientific units, and an index are provided below to facilitate use of this report by as wide an audience as possible. 


\title{
Annex II
}

\section{Glossary}

\author{
Editor: Alfons P. M. Baede (Netherlands) \\ Co-editors: Paul van der Linden (United Kingdom), Aviel Verbruggen (Belgium)
}

This Glossary is based on the glossaries published in the contributions of Working Groups of I, II and III to the IPCC Fourth Assessment Report. Additional work has been undertaken on additions, consistency and shortening of definitions to make this glossary more suitable to a wider audience.

The italics used have the following meaning: Glossary word reference; Glossary secondary reference (i.e. terms which are either contained in a glossary of the IPCC Working Group contributions to the AR4, or defined within the text of an entry of this glossary).

A.

\section{Abrupt climate change}

The nonlinearity of the climate system may lead to abrupt climate change, sometimes called rapid climate change, abrupt events or even surprises. The term abrupt often refers to time scales faster than the typical time scale of the responsible forcing. However, not all abrupt climate changes need be externally forced. Some possible abrupt events that have been proposed include a dramatic reorganisation of the thermohaline circulation, rapid deglaciation and massive melting of permafrost or increases in soil respiration leading to fast changes in the carbon cycle. Others may be truly unexpected, resulting from a strong, rapidly changing, forcing of a non-linear system.

\section{Absorption, scattering and emission of radiation}

Electromagnetic radiation may interact with matter, be it in the form of the atoms and molecules of a gas (e.g. the gases in the atmosphere) or in the form of particulate, solid or liquid, matter (e.g. aerosols), in various ways. Matter itself emits radiation in accordance with its composition and temperature. Radiation may be absorbed by matter, whereby the absorbed energy may be transferred or re-emitted. Finally, radiation may also be deflected from its original path (scattered) as a result of interaction with matter.

\section{Activities Implemented Jointly (AIJ)}

The pilot phase for Joint Implementation, as defined in Article 4.2(a) of the United Nations Framework Convention on Climate Change (UNFCCC) that allows for project activity among developed countries (and their companies) and between developed and developing countries (and their companies). AIJ is intended to allow parties to the UNFCCC to gain experience in jointly implemented projects. There is no credit for AIJ during the pilot phase. A decision remains on the future of AIJ projects and how they may relate to the Kyoto Mechanisms. As a simple form of tradable permits, AIJ and other market-based schemes represent potential mechanisms for stimulating additional resource flows for reducing emissions. See also Clean Development Mechanism, and Emissions Trading.

\section{Adaptation}

Initiatives and measures to reduce the vulnerability of natural and human systems against actual or expected climate change effects. Various types of adaptation exist, e.g. anticipatory and reactive, private and public, and autonomous and planned. Examples are raising river or coastal dikes, the substitution of more temperature-shock resistant plants for sensitive ones, etc.

\section{Adaptation benefits}

The avoided damage costs or the accrued benefits following the adoption and implementation of adaptation measures.

\section{Adaptation costs}

Costs of planning, preparing for, facilitating, and implementing adaptation measures, including transition costs.

\section{Adaptive capacity}

The whole of capabilities, resources and institutions of a country or region to implement effective adaptation measures.

\section{Aerosols}

A collection of airborne solid or liquid particles, with a typical size between 0.01 and 10 micrometer (a millionth of a meter) that reside in the atmosphere for at least several hours. Aerosols may be of either natural or anthropogenic origin. Aerosols may influence climate in several ways: directly through scattering and absorbing radiation, and indirectly through acting as cloud condensation nuclei or modifying the optical properties and lifetime of clouds.

\section{Afforestation}

Planting of new forests on lands that historically have not contained forests (for at least 50 years). For a discussion of the term forest and related terms such as afforestation, reforestation, and deforestation see the IPCC Report on Land Use, Land-Use Change and Forestry (IPCC, 2000). See also the Report on Definitions and Methodological Options to Inventory Emissions from Direct Human-induced Degradation of Forests and Devegetation of Other Vegetation Types (IPCC, 2003).

\section{Aggregate impacts}

Total impacts integrated across sectors and/or regions. The aggregation of impacts requires knowledge of (or assumptions about) the relative importance of impacts in different sectors and regions. Measures of aggregate impacts include, for example, the total number of people affected, or the total economic costs.

\section{Albedo}

The fraction of solar radiation reflected by a surface or object, often expressed as a percentage. Snow-covered surfaces have a high albedo, the surface albedo of soils ranges from high to low, and vegetation-covered surfaces and oceans have a low albedo. The Earth's planetary albedo varies mainly through varying cloudiness, snow, ice, leaf area and land cover changes.

\section{Albedo feedback}

A climate feedback involving changes in the Earth's albedo. It usually refers to changes in the cryosphere which has an albedo much larger $(\sim 0.8)$ than the average planetary albedo ( 0.3). In a warming climate, it is anticipated that the cryosphere would shrink, the Earth's overall albedo would decrease and more solar energy would be absorbed to warm the Earth still further.

\section{Algal bloom}

A reproductive explosion of algae in a lake, river, or ocean.

\section{Alpine}

The biogeographic zone made up of slopes above the tree line, characterised by the presence of rosette-forming herbaceous plants and low shrubby slowgrowing woody plants. 


\section{Annex I countries}

The group of countries included in Annex I (as amended in 1998) to the United Nations Framework Convention on Climate Change (UNFCCC), including all the OECD countries in the year 1990 and countries with economies in transition. Under Articles 4.2 (a) and 4.2 (b) of the Convention, Annex I countries committed themselves specifically to the aim of returning individually or jointly to their 1990 levels of greenhouse gas emissions by the year 2000. By default, the other countries are referred to as Non-Annex I countries. For a list of Annex I countries, see http://unfccc.int.

\section{Annex II countries}

The group of countries included in Annex II to the United Nations Framework Convention on Climate Change (UNFCCC), including all OECD countries in the year 1990. Under Article 4.2 (g) of the Convention, these countries are expected to provide financial resources to assist developing countries to comply with their obligations, such as preparing national reports. Annex II countries are also expected to promote the transfer of environmentally sound technologies to developing countries. For a list of Annex II countries, see http://unfccc.int.

\section{Annex B countries}

The countries included in Annex B to the Kyoto Protocol that have agreed to a target for their greenhouse-gas emissions, including all the Annex I countries (as amended in 1998) except for Turkey and Belarus. For a list of Annex I countries, see http://unfccc.int. See Kyoto Protocol

\section{Anthropogenic}

Resulting from or produced by human beings.

\section{Anthropogenic emissions}

Emissions of greenhouse gases, greenhouse gas precursors, and aerosols associated with human activities, including the burning of fossil fuels, deforestation, land-use changes, livestock, fertilisation, etc.

\section{Arid region}

A land region of low rainfall, where low is widely accepted to be $<250 \mathrm{~mm}$ precipitation per year.

\section{Atmosphere}

The gaseous envelope surrounding the Earth. The dry atmosphere consists almost entirely of nitrogen ( $78.1 \%$ volume mixing ratio) and oxygen $(20.9 \%$ volume mixing ratio), together with a number of trace gases, such as argon $(0.93 \%$ volume mixing ratio), helium and radiatively active greenhouse gases such as carbon dioxide ( $0.035 \%$ volume mixing ratio) and ozone. In addition, the atmosphere contains the greenhouse gas water vapour, whose amounts are highly variable but typically around $1 \%$ volume mixing ratio. The atmosphere also contains clouds and aerosols.

\section{Attribution}

See Detection and attribution.

\section{B.}

\section{Barrier}

Any obstacle to reaching a goal, adaptation or mitigation potential that can be overcome or attenuated by a policy, programme, or measure. Barrier removal includes correcting market failures directly or reducing the transactions costs in the public and private sectors by e.g. improving institutional capacity, reducing risk and uncertainty, facilitating market transactions, and enforcing regulatory policies.

\section{Baseline}

Reference for measurable quantities from which an alternative outcome can be measured, e.g. a non-intervention scenario used as a reference in the analysis of intervention scenarios.

\section{Basin}

The drainage area of a stream, river, or lake.

\section{Biodiversity}

The total diversity of all organisms and ecosystems at various spatial scales (from genes to entire biomes).

\section{Biofuel}

A fuel produced from organic matter or combustible oils produced by plants. Examples of biofuel include alcohol, black liquor from the paper-manufacturing process, wood, and soybean oil.

\section{Biomass}

The total mass of living organisms in a given area or volume; recently dead plant material is often included as dead biomass. The quantity of biomass is expressed as a dry weight or as the energy, carbon, or nitrogen content.

\section{Biome}

A major and distinct regional element of the biosphere, typically consisting of several ecosystems (e.g. forests, rivers, ponds, swamps within a region of similar climate). Biomes are characterised by typical communities of plants and animals.

\section{Biosphere (terrestrial and marine)}

The part of the Earth system comprising all ecosystems and living organisms, in the atmosphere, on land (terrestrial biosphere) or in the oceans (marine biosphere), including derived dead organic matter, such as litter, soil organic matter and oceanic detritus.

\section{Boreal forest}

Forests of pine, spruce, fir, and larch stretching from the east coast of Canada westward to Alaska and continuing from Siberia westward across the entire extent of Russia to the European Plain.

\section{Borehole temperature}

Borehole temperatures are measured in boreholes of tens to hundreds of meters depth into the subsurface of the Earth. Borehole temperature depth profiles are commonly used to infer time variations in the ground surface temperature on centennial time scales.

\section{Bottom-up models}

Bottom-up models represent reality by aggregating characteristics of specific activities and processes, considering technological, engineering and cost details. See also Top-down models.

C.

\section{Carbon (Dioxide) Capture and Storage (CCS)}

A process consisting of separation of carbon dioxide from industrial and energy-related sources, transport to a storage location, and long-term isolation from the atmosphere.

\section{Carbon cycle}

The term used to describe the flow of carbon (in various forms, e.g. as carbon dioxide) through the atmosphere, ocean, terrestrial biosphere and lithosphere.

\section{Carbon dioxide $\left(\mathrm{CO}_{2}\right)$}

A naturally occurring gas, also a by-product of burning fossil fuels from fossil carbon deposits, such as oil, gas and coal, of burning biomass and of land use changes and other industrial processes. It is the principal anthropogenic greenhouse gas that affects the Earth's radiative balance. It is the reference gas against which other greenhouse gases are measured and therefore has a Global Warming Potential of 1.

\section{Carbon dioxide $\left(\mathrm{CO}_{2}\right)$ fertilisation}

The enhancement of the growth of plants as a result of increased atmospheric carbon dioxide $\left(\mathrm{CO}_{2}\right)$ concentration. Depending on their mechanism of photosynthesis, certain types of plants are more sensitive to changes in atmospheric $\mathrm{CO}_{2}$ concentration. 


\section{Carbon intensity}

The amount of emission of carbon dioxide per unit of Gross Domestic Product.

\section{Carbon leakage}

The part of emissions reductions in Annex $B$ countries that may be offset by an increase of the emissions in the non-constrained countries above their baseline levels. This can occur through (1) relocation of energy-intensive production in non-constrained regions; (2) increased consumption of fossil fuels in these regions through decline in the international price of oil and gas triggered by lower demand for these energies; and (3) changes in incomes (thus in energy demand) because of better terms of trade.

\section{Carbon sequestration}

See Uptake

Catchment

An area that collects and drains rainwater.

\section{Chlorofluorocarbons (CFCs)}

See Halocarbons

\section{Clean Development Mechanism (CDM)}

Defined in Article 12 of the Kyoto Protocol, the CDM is intended to meet two objectives: (1) to assist parties not included in Annex $I$ in achieving sustainable development and in contributing to the ultimate objective of the convention; and (2) to assist parties included in Annex I in achieving compliance with their quantified emission limitation and reduction commitments. Certified Emission Reduction Units from CDM projects undertaken in non-Annex I countries that limit or reduce greenhouse gas emissions, when certified by operational entities designated by Conference of the Parties/Meeting of the Parties, can be accrued to the investor (government or industry) from parties in Annex B. A share of the proceeds from the certified project activities is used to cover administrative expenses as well as to assist developing country parties that are particularly vulnerable to the adverse effects of climate change to meet the costs of adaptation.

\section{Climate}

Climate in a narrow sense is usually defined as the average weather, or more rigorously, as the statistical description in terms of the mean and variability of relevant quantities over a period of time ranging from months to thousands or millions of years. The classical period for averaging these variables is 30 years, as defined by the World Meteorological Organization. The relevant quantities are most often surface variables such as temperature, precipitation and wind. Climate in a wider sense is the state, including a statistical description, of the climate system. In various parts of this report different averaging periods, such as a period of 20 years, are also used.

\section{Climate-carbon cycle coupling}

Future climate change induced by atmospheric emissions of greenhouse gases will impact on the global carbon cycle. Changes in the global carbon cycle in turn will influence the fraction of anthropogenic greenhouse gases that remains in the atmosphere, and hence the atmospheric concentrations of greenhouse gases, resulting in further climate change. This feedback is called climate-carbon cycle coupling. The first generation coupled climate-carbon cycle models indicates that global warming will increase the fraction of anthropogenic $\mathrm{CO}_{2}$ that remains in the atmosphere.

\section{Climate change}

Climate change refers to a change in the state of the climate that can be identified (e.g., by using statistical tests) by changes in the mean and/or the variability of its properties, and that persists for an extended period, typically decades or longer. Climate change may be due to natural internal processes or external forcings, or to persistent anthropogenic changes in the composition of the atmosphere or in land use. Note that the United Nations Framework Convention on Climate Change (UNFCCC), in its Article 1, defines climate change as: 'a change of climate which is attributed directly or indirectly to human activity that alters the composition of the global atmosphere and which is in addition to natural climate variability observed over comparable time periods'. The UNFCCC thus makes a distinction between climate change attributable to human activities altering the atmospheric composition, and climate variability attributable to natural causes. See also Climate variability; Detection and Attribution.

\section{Climate feedback}

An interaction mechanism between processes in the climate system is called a climate feedback when the result of an initial process triggers changes in a second process that in turn influences the initial one. A positive feedback intensifies the original process, and a negative feedback reduces it.

\section{Climate model}

A numerical representation of the climate system based on the physical, chemical and biological properties of its components, their interactions and feedback processes, and accounting for all or some of its known properties. The climate system can be represented by models of varying complexity, that is, for any one component or combination of components a spectrum or hierarchy of models can be identified, differing in such aspects as the number of spatial dimensions, the extent to which physical, chemical or biological processes are explicitly represented, or the level at which empirical parametrisations are involved. Coupled Atmosphere-Ocean General Circulation Models (AOGCMs) provide a representation of the climate system that is near the most comprehensive end of the spectrum currently available. There is an evolution towards more complex models with interactive chemistry and biology (see WGI Chapter 8). Climate models are applied as a research tool to study and simulate the climate, and for operational purposes, including monthly, seasonal and interannual climate predictions.

\section{Climate prediction}

A climate prediction or climate forecast is the result of an attempt to produce an estimate of the actual evolution of the climate in the future, for example, at seasonal, interannual or long-term time scales. Since the future evolution of the climate system may be highly sensitive to initial conditions, such predictions are usually probabilistic in nature. See also $\mathrm{Cli}$ mate projection, climate scenario.

\section{Climate projection}

A projection of the response of the climate system to emission or concentration scenarios of greenhouse gases and aerosols, or radiative forcing scenarios, often based upon simulations by climate models. Climate projections are distinguished from climate predictions in order to emphasise that climate projections depend upon the emission/concentration/radiative forcing scenario used, which are based on assumptions concerning, for example, future socioeconomic and technological developments that may or may not be realised and are therefore subject to substantial uncertainty.

\section{Climate response}

See Climate sensitivity

\section{Climate scenario}

A plausible and often simplified representation of the future climate, based on an internally consistent set of climatological relationships that has been constructed for explicit use in investigating the potential consequences of anthropogenic climate change, often serving as input to impact models. Climate projections often serve as the raw material for constructing climate scenarios, but climate scenarios usually require additional information such as about the observed current climate. A climate change scenario is the difference between a climate scenario and the current climate.

\section{Climate sensitivity}

In IPCC reports, equilibrium climate sensitivity refers to the equilibrium change in the annual mean global surface temperature following a doubling of the atmospheric equivalent carbon dioxide concentration. Due to computational constraints, the equilibrium climate sensitivity in a climate model is usually estimated by running an atmospheric general circulation model coupled to a mixed-layer ocean model, because equilibrium climate sensitivity is largely determined by atmospheric processes. Efficient models can be run to equilibrium with a dynamic ocean. 
The transient climate response is the change in the global surface temperature, averaged over a 20 -year period, centred at the time of atmospheric carbon dioxide doubling, that is, at year 70 in a $1 \% / \mathrm{yr}$ compound carbon dioxide increase experiment with a global coupled climate model. It is a measure of the strength and rapidity of the surface temperature response to greenhouse gas forcing.

\section{Climate shift}

An abrupt shift or jump in mean values signalling a change in climate regime (see Patterns of climate variability). Most widely used in conjunction with the 1976/1977 climate shift that seems to correspond to a change in El Niño-Southern Oscillation behaviour.

\section{Climate system}

The climate system is the highly complex system consisting of five major components: the atmosphere, the hydrosphere, the cryosphere, the land surface and the biosphere, and the interactions between them. The climate system evolves in time under the influence of its own internal dynamics and because of external forcings such as volcanic eruptions, solar variations and anthropogenic forcings such as the changing composition of the atmosphere and land-use change.

\section{Climate variability}

Climate variability refers to variations in the mean state and other statistics (such as standard deviations, the occurrence of extremes, etc.) of the climate on all spatial and temporal scales beyond that of individual weather events. Variability may be due to natural internal processes within the climate system (internal variability), or to variations in natural or anthropogenic external forcing (external variability). See also Climate change.

\section{Cloud feedback}

A climate feedback involving changes in any of the properties of clouds as a response to other atmospheric changes. Understanding cloud feedbacks and determining their magnitude and sign require an understanding of how a change in climate may affect the spectrum of cloud types, the cloud fraction and height, and the radiative properties of clouds, and an estimate of the impact of these changes on the Earth's radiation budget. At present, cloud feedbacks remain the largest source of uncertainty in climate sensitivity estimates. See also Radiative forcing.

\section{$\mathrm{CO}_{2}$-equivalent}

See Box "Carbon dioxide-equivalent $\left(\mathrm{CO}_{2}\right.$-eq) emissions and concentrations" in Topic 2 of the Synthesis Report and Working Group I Chapter 2.10 .

\section{$\mathrm{CO}_{2}$-fertilization}

See Carbon dioxide fertilization.

\section{Co-benefits}

The benefits of policies implemented for various reasons at the same time, acknowledging that most policies designed to address greenhouse gas mitigation have other, often at least equally important, rationales (e.g., related to objectives of development, sustainability, and equity).

\section{Combined Heat and Power (CHP)}

The use of waste heat from thermal electricity generation plants. The heat is e.g. condensing heat from steam turbines or hot flue gases exhausted from gas turbines, for industrial use, buildings or district heating. Also called co-generation.

\section{Compliance}

Compliance is whether and to what extent countries do adhere to the provisions of an accord. Compliance depends on implementing policies ordered, and on whether measures follow up the policies. Compliance is the degree to which the actors whose behaviour is targeted by the agreement, local government units, corporations, organisations, or individuals, conform to the implementing obligations. See also Implementation.

\section{Confidence}

The level of confidence in the correctness of a result is expressed in this report, using a standard terminology defined as follows:

\begin{tabular}{|l|l|}
\hline Terminology & Degree of confidence in being correct \\
\hline Very high confidence & At least 9 out of 10 chance of being correct \\
High confidence & About 8 out of 10 chance \\
Medium confidence & About 5 out of 10 chance \\
Low confidence & About 2 out of 10 chance \\
Very low confidence & Less than 1 out of 10 chance \\
\hline
\end{tabular}

See also Likelihood; Uncertainty

\section{Coral}

The term coral has several meanings, but is usually the common name for the Order Scleractinia, all members of which have hard limestone skeletons, and which are divided into reef-building and non-reef-building, or cold- and warm-water corals. See Coral bleaching; Coral reefs

\section{Coral bleaching}

The paling in colour which results if a coral loses its symbiotic, energyproviding, organisms.

\section{Coral reefs}

Rock-like limestone structures built by corals along ocean coasts (fringing reefs) or on top of shallow, submerged banks or shelves (barrier reefs, atolls), most conspicuous in tropical and subtropical oceans.

\section{Cost}

The consumption of resources such as labour time, capital, materials, fuels, etc. as a consequence of an action. In economics all resources are valued at their opportunity cost, being the value of the most valuable alternative use of the resources. Costs are defined in a variety of ways and under a variety of assumptions that affect their value. Cost types include: administrative costs, damage costs (to ecosystems, people and economies due to negative effects from climate change), and implementation costs of changing existing rules and regulation, capacity building efforts, information, training and education, etc. Private costs are carried by individuals, companies or other private entities that undertake the action, whereas social costs include also the external costs on the environment and on society as a whole. The negative of costs are benefits (also sometimes called negative costs). Costs minus benefits are net costs.

\section{Cryosphere}

The component of the climate system consisting of all snow, ice and frozen ground (including permafrost) on and beneath the surface of the Earth and ocean. See also Glacier; Ice sheet.

D.

\section{Deforestation}

Conversion of forest to non-forest. For a discussion of the term forest and related terms such as afforestation, reforestation, and deforestation see the IPCC Report on Land Use, Land-Use Change and Forestry (IPCC, 2000). See also the Report on Definitions and Methodological Options to Inventory Emissions from Direct Human-induced Degradation of Forests and Devegetation of Other Vegetation Types (IPCC, 2003).

\section{Demand-side management (DSM)}

Policies and programmes for influencing the demand for goods and/or services. In the energy sector, DSM aims at reducing the demand for electricity and energy sources. DSM helps to reduce greenhouse gas emissions.

\section{Detection and attribution}

Climate varies continually on all time scales. Detection of climate change is the process of demonstrating that climate has changed in some defined statistical sense, without providing a reason for that change. Attribution of causes of climate change is the process of establishing the most likely causes for the detected change with some defined level of confidence. 


\section{Development path or pathway}

An evolution based on an array of technological, economic, social, institutional, cultural, and biophysical characteristics that determine the interactions between natural and human systems, including production and consumption patterns in all countries, over time at a particular scale. Alternative development paths refer to different possible trajectories of development, the continuation of current trends being just one of the many paths.

\section{Discounting}

A mathematical operation making monetary (or other) amounts received or expended at different points in time (years) comparable across time. The operator uses a fixed or possibly time-varying discount rate $(>0)$ from year to year that makes future value worth less today. In a descriptive discounting approach one accepts the discount rates people (savers and investors) actually apply in their day-to-day decisions (private discount rate). In a prescriptive (ethical or normative) discounting approach the discount rate is fixed from a social perspective, e.g. based on an ethical judgement about the interests of future generations (social discount rate).

\section{Discount rate}

See Discounting

\section{Drought}

In general terms, drought is a 'prolonged absence or marked deficiency of precipitation', a 'deficiency that results in water shortage for some activity or for some group', or a 'period of abnormally dry weather sufficiently prolonged for the lack of precipitation to cause a serious hydrological imbalance' (Heim, 2002). Drought has been defined in a number of ways. Agricultural drought relates to moisture deficits in the topmost 1 metre or so of soil (the root zone) that affect crops, meteorological drought is mainly a prolonged deficit of precipitation, and hydrologic drought is related to below-normal streamflow, lake and groundwater levels. A megadrought is a longdrawn out and pervasive drought, lasting much longer than normal, usually a decade or more.

\section{Dynamical ice discharge}

Discharge of ice from ice sheets or ice caps caused by the dynamics of the ice sheet or ice cap (e.g. in the form of glacier flow, ice streams and calving icebergs) rather than by melt or runoff.

E.

Economic (mitigation) potential

See Mitigation potential.

\section{Economies in Transition (EITs)}

Countries with their economies changing from a planned economic system to a market economy.

\section{Ecosystem}

A system of living organisms interacting with each other and their physical environment. The boundaries of what could be called an ecosystem are somewhat arbitrary, depending on the focus of interest or study. Thus, the extent of an ecosystem may range from very small spatial scales to, ultimately, the entire Earth.

\section{El Niño-Southern Oscillation (ENSO)}

The term El Niño was initially used to describe a warm-water current that periodically flows along the coast of Ecuador and Perú, disrupting the local fishery. It has since become identified with a basinwide warming of the tropical Pacific east of the dateline. This oceanic event is associated with a fluctuation of a global-scale tropical and subtropical surface pressure pattern called the Southern Oscillation. This coupled atmosphereocean phenomenon, with preferred time scales of two to about seven years, is collectively known as El Niño-Southern Oscillation, or ENSO. It is often measured by the surface pressure anomaly difference between Darwin and Tahiti and the sea surface temperatures in the central and eastern equatorial Pacific. During an ENSO event, the prevailing trade winds weaken, reducing upwelling and altering ocean currents such that the sea surface temperatures warm, further weakening the trade winds. This event has a great impact on the wind, sea surface temperature and precipitation patterns in the tropical Pacific. It has climatic effects throughout the Pacific region and in many other parts of the world, through global teleconnections. The cold phase of ENSO is called La Niña.

\section{Emission scenario}

A plausible representation of the future development of emissions of substances that are potentially radiatively active (e.g., greenhouse gases, aerosols), based on a coherent and internally consistent set of assumptions about driving forces (such as demographic and socioeconomic development, technological change) and their key relationships. Concentration scenarios, derived from emission scenarios, are used as input to a climate model to compute climate projections. In IPCC (1992) a set of emission scenarios was presented which were used as a basis for the climate projections in IPCC (1996). These emission scenarios are referred to as the IS92 scenarios. In the IPCC Special Report on Emission Scenarios (Nakičenovič and Swart, 2000) new emission scenarios, the so-called SRES scenarios, were published. For the meaning of some terms related to these scenarios, see SRES scenarios.

\section{Emission(s) trading}

A market-based approach to achieving environmental objectives. It allows those reducing greenhouse gas emissions below their emission cap to use or trade the excess reductions to offset emissions at another source inside or outside the country. In general, trading can occur at the intra-company, domestic, and international levels. The Second Assessment Report by the IPCC adopted the convention of using permits for domestic trading systems and quotas for international trading systems. Emissions trading under Article 17 of the Kyoto Protocol is a tradable quota system based on the assigned amounts calculated from the emission reduction and limitation commitments listed in Annex B of the Protocol.

\section{Emission trajectory}

A projected development in time of the emission of a greenhouse gas or group of greenhouse gases, aerosols and greenhouse gas precursors.

\section{Energy}

The amount of work or heat delivered. Energy is classified in a variety of types and becomes useful to human ends when it flows from one place to another or is converted from one type into another. Primary energy (also referred to as energy sources) is the energy embodied in natural resources (e.g., coal, crude oil, natural gas, uranium) that has not undergone any anthropogenic conversion. This primary energy needs to be converted and transported to become usable energy (e.g. light). Renewable energy is obtained from the continuing or repetitive currents of energy occurring in the natural environment, and includes non-carbon technologies such as solar energy, hydropower, wind, tide and waves, and geothermal heat, as well as carbon neutral technologies such as biomass. Embodied energy is the energy used to produce a material substance (such as processed metals, or building materials), taking into account energy used at the manufacturing facility (zero order), energy used in producing the materials that are used in the manufacturing facility (first order), and so on.

\section{Energy balance}

The difference between the total incoming and total outgoing energy in the climate system. If this balance is positive, warming occurs; if it is negative, cooling occurs. Averaged over the globe and over long time periods, this balance must be zero. Because the climate system derives virtually all its energy from the Sun, zero balance implies that, globally, the amount of incoming solar radiation on average must be equal to the sum of the outgoing reflected solar radiation and the outgoing thermal infrared radiation emitted by the climate system. A perturbation of this global radiation balance, be it anthropogenic or natural, is called radiative forcing.

\section{Energy efficiency}

Ratio of useful energy output of a system, conversion process or activity, to its energy input. 


\section{Energy intensity}

Energy intensity is the ratio of energy use to economic or physical output. At the national level, energy intensity is the ratio of total primary energy use or final energy use to Gross Domestic Product. At the activity level, one can also use physical quantities in the denominator, e.g. litre fuel/ vehicle $\mathrm{km}$.

\section{Equivalent carbon dioxide concentration}

See Box "Carbon dioxide-equivalent $\left(\mathrm{CO}_{2}\right.$-eq) emissions and concentrations" in Topic 2 of the Synthesis Report.

\section{Equivalent carbon dioxide emission}

See Box "Carbon dioxide-equivalent $\left(\mathrm{CO}_{2}\right.$-eq) emissions and concentrations" in Topic 2 of the Synthesis Report and Working Group I Chapter 2.10 .

\section{Erosion}

The process of removal and transport of soil and rock by weathering, mass wasting, and the action of streams, glaciers, waves, winds, and underground water.

\section{Evapotranspiration}

The combined process of water evaporation from the Earth's surface and transpiration from vegetation.

\section{External forcing}

External forcing refers to a forcing agent outside the climate system causing a change in the climate system. Volcanic eruptions, solar variations and anthropogenic changes in the composition of the atmosphere and landuse change are external forcings.

\section{Extinction}

The complete disappearance of an entire biological species.

\section{Extreme weather event}

An event that is rare at a particular place and time of year. Definitions of "rare" vary, but an extreme weather event would normally be as rare as or rarer than the 10th or 90th percentile of the observed probability density function. By definition, the characteristics of what is called extreme weather may vary from place to place in an absolute sense. Single extreme events cannot be simply and directly attributed to anthropogenic climate change, as there is always a finite chance the event in question might have occurred naturally. When a pattern of extreme weather persists for some time, such as a season, it may be classed as an extreme climate event, especially if it yields an average or total that is itself extreme (e.g., drought or heavy rainfall over a season).

\section{F.}

F-gases

This term refers to the groups of gases hydrofluorocarbons, perfluorocarbons, and sulphurhexafluoride, which are covered under the Kyoto Protocol.

\section{Feedback}

See Climate feedback.

\section{Food security}

A situation that exists when people have secure access to sufficient amounts of safe and nutritious food for normal growth, development and an active and healthy life. Food insecurity may be caused by the unavailability of food, insufficient purchasing power, inappropriate distribution, or inadequate use of food at the household level.

\section{Forcing}

See External forcing

\section{Forecast}

See Climate forecast; Climate projection; Projection.

\section{Forest}

A vegetation type dominated by trees. Many definitions of the term forest are in use throughout the world, reflecting wide differences in biogeophysical conditions, social structure, and economics. Particular criteria apply under the Kyoto Protocol. For a discussion of the term forest and related terms such as afforestation, reforestation, and deforestation see the IPCC Special Report on Land Use, Land-Use Change, and Forestry (IPCC, 2000). See also the Report on Definitions and Methodological Options to Inventory Emissions from Direct Human-induced Degradation of Forests and Devegetation of Other Vegetation Types (IPCC, 2003)

\section{Fossil fuels}

Carbon-based fuels from fossil hydrocarbon deposits, including coal, peat, oil, and natural gas.

\section{Framework Convention on Climate Change}

See United Nations Framework Convention on Climate Change (UNFCCC).

\section{Frozen ground}

Soil or rock in which part or all of the pore water is frozen (Van Everdingen, 1998). Frozen ground includes permafrost. Ground that freezes and thaws annually is called seasonally frozen ground.

\section{Fuel cell}

A fuel cell generates electricity in a direct and continuous way from the controlled electrochemical reaction of hydrogen or another fuel and oxygen. With hydrogen as fuel it emits only water and heat (no carbon dioxide) and the heat can be utilised. See Combined Heat and Power.

\section{Fuel switching}

In general this is substituting fuel A for fuel $\mathrm{B}$. In the climate change discussion it is implicit that fuel A has a lower carbon content than fuel $\mathrm{B}$, e.g. natural gas for coal.

G.

\section{Glacial lake}

A lake formed by glacier meltwater, located either at the front of a glacier (known as a proglacial lake), on the surface of a glacier (supraglacial lake), within the glacier (englacial lake) or at the glacier bed (subglacial lake).

\section{Glacier}

A mass of land ice which flows downhill under gravity (through internal deformation and/or sliding at the base) and is constrained by internal stress and friction at the base and sides. A glacier is maintained by accumulation of snow at high altitudes, balanced by melting at low altitudes or discharge into the sea. See Mass balance

\section{Global surface temperature}

The global surface temperature is an estimate of the global mean surface air temperature. However, for changes over time, only anomalies, as departures from a climatology, are used, most commonly based on the areaweighted global average of the sea surface temperature anomaly and land surface air temperature anomaly.

\section{Global Warming Potential (GWP)}

An index, based upon radiative properties of well mixed greenhouse gases, measuring the radiative forcing of a unit mass of a given well mixed greenhouse gas in today's atmosphere integrated over a chosen time horizon, relative to that of carbon dioxide. The GWP represents the combined effect of the differing times these gases remain in the atmosphere and their relative effectiveness in absorbing outgoing thermal infrared radiation. The Kyoto Protocol is based on GWPs from pulse emissions over a 100year time frame.

\section{Greenhouse effect}

Greenhouse gases effectively absorb thermal infrared radiation, emitted by the Earth's surface, by the atmosphere itself due to the same gases, and 
by clouds. Atmospheric radiation is emitted to all sides, including downward to the Earth's surface. Thus greenhouse gases trap heat within the surface-troposphere system. This is called the greenhouse effect.Thermal infrared radiation in the troposphere is strongly coupled to the temperature of the atmosphere at the altitude at which it is emitted. In the troposphere, the temperature generally decreases with height. Effectively, infrared radiation emitted to space originates from an altitude with a temperature of, on average, $-19^{\circ} \mathrm{C}$, in balance with the net incoming solar radiation, whereas the Earth's surface is kept at a much higher temperature of, on average,$+14^{\circ} \mathrm{C}$. An increase in the concentration of greenhouse gases leads to an increased infrared opacity of the atmosphere, and therefore to an effective radiation into space from a higher altitude at a lower temperature. This causes a radiative forcing that leads to an enhancement of the greenhouse effect, the so-called enhanced greenhouse effect.

\section{Greenhouse gas (GHG)}

Greenhouse gases are those gaseous constituents of the atmosphere, both natural and anthropogenic, that absorb and emit radiation at specific wavelengths within the spectrum of thermal infrared radiation emitted by the Earth's surface, the atmosphere itself, and by clouds. This property causes the greenhouse effect. Water vapour $\left(\mathrm{H}_{2} \mathrm{O}\right)$, carbon dioxide $\left(\mathrm{CO}_{2}\right)$, nitrous oxide $\left(\mathrm{N}_{2} \mathrm{O}\right)$, methane $\left(\mathrm{CH}_{4}\right)$ and ozone $\left(\mathrm{O}_{3}\right)$ are the primary greenhouse gases in the Earth's atmosphere. Moreover, there are a number of entirely human-made greenhouse gases in the atmosphere, such as the halocarbons and other chlorine and bromine containing substances, dealt with under the Montreal Protocol. Beside $\mathrm{CO}_{2}, \mathrm{~N}_{2} \mathrm{O}$ and $\mathrm{CH}_{4}$, the Kyoto Protocol deals with the greenhouse gases sulphur hexafluoride $\left(\mathrm{SF}_{6}\right)$, hydrofluorocarbons (HFCs) and perfluorocarbons (PFCs).

\section{Gross Domestic Product (GDP)}

Gross Domestic Product (GDP) is the monetary value of all goods and services produced within a nation.

H.

\section{Halocarbons}

A collective term for the group of partially halogenated organic species, including the chlorofluorocarbons ( $\mathrm{CFCs}$ ), hydrochlorofluorocarbons (HCFCs), hydrofluorocarbons (HFCs), halons, methyl chloride, methyl bromide, etc. Many of the halocarbons have large Global Warming Potentials. The chlorine and bromine containing halocarbons are also involved in the depletion of the ozone layer.

\section{Human system}

Any system in which human organisations play a major role. Often, but not always, the term is synonymous with society or social system e.g., agricultural system, political system, technological system, economic system; all are human systems in the sense applied in the Fourth Assessment Report.

\section{Hydrochlorofluorocarbons (HCFCs)}

See Halocarbons

\section{Hydrofluorocarbons (HFCs)}

One of the six greenhouse gases or groups of greenhouse gases to be curbed under the Kyoto Protocol. They are produced commercially as a substitute for chlorofluorocarbons. HFCs largely are used in refrigeration and semiconductor manufacturing. See Halocarbons

\section{Hydrosphere}

The component of the climate system comprising liquid surface and subterranean water, such as oceans, seas, rivers, fresh water lakes, underground water, etc.

\section{Hydrological cycle}

The cycle in which water evaporates from the oceans and the land surface, is carried over the Earth in atmospheric circulation as water vapour, condensates to form clouds, precipitates again as rain or snow, is intercepted by trees and vegetation, provides runoff on the land surface, infiltrates into soils, recharges groundwater, discharges into streams, and ultimately, flows out into the oceans, from which it will eventually evaporate again (AMS, 2000). The various systems involved in the hydrological cycle are usually referred to as hydrological systems.

\section{Hydrological systems}

See Hydrological cycle

I.

\section{Ice cap}

A dome shaped ice mass, usually covering a highland area, which is considerably smaller in extent than an ice sheet.

\section{Ice core}

A cylinder of ice drilled out of a glacier or ice sheet.

\section{Ice sheet}

A mass of land ice that is sufficiently deep to cover most of the underlying bedrock topography, so that its shape is mainly determined by its dynamics (the flow of the ice as it deforms internally and/or slides at its base). An ice sheet flows outwards from a high central ice plateau with a small average surface slope. The margins usually slope more steeply, and most ice is discharged through fast-flowing ice streams or outlet glaciers, in some cases into the sea or into ice shelves floating on the sea. There are only three large ice sheets in the modern world, one on Greenland and two on Antarctica, the East and West Antarctic Ice Sheet, divided by the Transantarctic Mountains. During glacial periods there were others.

\section{(Climate change) Impact assessment}

The practice of identifying and evaluating, in monetary and/or non-monetary terms, the effects of climate change on natural and human systems.

\section{(Climate change) Impacts}

The effects of climate change on natural and human systems. Depending on the consideration of adaptation, one can distinguish between potential impacts and residual impacts:

- Potential impacts: all impacts that may occur given a projected change in climate, without considering adaptation.

- Residual impacts: the impacts of climate change that would oc cur after adaptation.

See also aggregate impacts, market impacts, and non-market impacts.

\section{Implementation}

Implementation describes the actions taken to meet commitments under a treaty and encompasses legal and effective phases.

Legal implementation refers to legislation, regulations, judicial decrees, including other actions such as efforts to administer progress which governments take to translate international accords into domestic law and policy. Effective implementation needs policies and programmes that induce changes in the behaviour and decisions of target groups. Target groups then take effective measures of mitigation and adaptation. See also Compliance.

\section{Indigenous peoples}

No internationally accepted definition of indigenous peoples exists. Common characteristics often applied under international law, and by United Nations agencies to distinguish indigenous peoples include: residence within or attachment to geographically distinct traditional habitats, ancestral territories, and their natural resources; maintenance of cultural and social identities, and social, economic, cultural and political institutions separate from mainstream or dominant societies and cultures; descent from population groups present in a given area, most frequently before modern states or territories were created and current borders defined; and self-identification as being part of a distinct indigenous cultural group, and the desire to preserve that cultural identity.

\section{Induced technological change}

See technological change. 


\section{Industrial revolution}

A period of rapid industrial growth with far-reaching social and economic consequences, beginning in Britain during the second half of the eighteenth century and spreading to Europe and later to other countries including the United States. The invention of the steam engine was an important trigger of this development. The industrial revolution marks the beginning of a strong increase in the use of fossil fuels and emission of, in particular, fossil carbon dioxide. In this Report the terms pre-industrial and industrial refer, somewhat arbitrarily, to the periods before and after 1750, respectively.

\section{Inertia}

In the context of climate change mitigation, inertia relates to the difficulty of change resulting from pre-existing conditions within society such as physical man-made capital, natural capital, and social non-physical capital, including institutions, regulations, and norms. Existing structures lock in societies making change more difficult.

In the context of the climate system, inertia relates to the delay in climate change after an external forcing has been applied, and to the continuation of climate change even after the external forcing has been stabilised.

\section{Infectious disease}

Any disease caused by microbial agents that can be transmitted from one person to another or from animals to people. This may occur by direct physical contact, by handling of an object that has picked up infective organisms, through a disease carrier, via contaminated water, or by spread of infected droplets coughed or exhaled into the air.

\section{Infrastructure}

The basic equipment, utilities, productive enterprises, installations, and services essential for the development, operation, and growth of an organization, city, or nation.

\section{Integrated assessment}

A method of analysis that combines results and models from the physical, biological, economic and social sciences, and the interactions between these components in a consistent framework to evaluate the status and the consequences of environmental change and the policy responses to it. Models used to carry out such analysis are called Integrated Assessment Models.

\section{Integrated water resources management (IWRM)}

The prevailing concept for water management which, however, has not been defined unambiguously. IWRM is based on four principles that were formulated by the International Conference on Water and the Environment in Dublin, 1992: 1) fresh water is a finite and vulnerable resource, essential to sustain life, development and the environment; 2) water development and management should be based on a participatory approach, involving users, planners and policymakers at all levels; 3 ) women play a central part in the provision, management and safeguarding of water; 4) water has an economic value in all its competing uses and should be recognised as an economic good.

\section{Interglacials}

The warm periods between ice age glaciations. The previous interglacial, dated approximately from 129,000 to 116,000 years ago, is referred to as Last Interglacial. (AMS, 2000)

\section{J.}

\section{Joint Implementation (JI)}

A market-based implementation mechanism defined in Article 6 of the Kyoto Protocol, allowing Annex I countries or companies from these countries to implement projects jointly that limit or reduce emissions or enhance sinks, and to share the Emissions Reduction Units. JI activity is also permitted in Article 4.2(a) of the United Nations Framework Convention on Climate Change (UNFCCC). See also Kyoto Mechanisms; Activities Implemented Jointly.

\section{K.}

Kyoto Mechanisms (also called Flexibility Mechanisms)

Economic mechanisms based on market principles that parties to the Kyoto Protocol can use in an attempt to lessen the potential economic impacts of greenhouse gas emission-reduction requirements. They include Joint Implementation (Article 6), Clean Development Mechanism (Article 12), and Emissions Trading (Article 17).

\section{Kyoto Protocol}

The Kyoto Protocol to the United Nations Framework Convention on Climate Change (UNFCCC) was adopted in 1997 in Kyoto, Japan, at the Third Session of the Conference of the Parties (COP) to the UNFCCC. It contains legally binding commitments, in addition to those included in the UNFCCC. Countries included in Annex $B$ of the Protocol (most Organization for Economic Cooperation and Development countries and countries with economies in transition) agreed to reduce their anthropogenic greenhouse gas emissions (carbon dioxide, methane, nitrous oxide, hydrofluorocarbons, perfluorocarbons, and sulphur hexafluoride) by at least 5\% below 1990 levels in the commitment period 2008 to 2012. The Kyoto Protocol entered into force on 16 February 2005.

\section{L.}

\section{Land use and Land-use change}

Land use refers to the total of arrangements, activities and inputs undertaken in a certain land cover type (a set of human actions). The term land use is also used in the sense of the social and economic purposes for which land is managed (e.g., grazing, timber extraction, and conservation).

Land-use change refers to a change in the use or management of land by humans, which may lead to a change in land cover. Land cover and landuse change may have an impact on the surface albedo, evapotranspiration, sources and sinks of greenhouse gases, or other properties of the climate system and may thus have a radiative forcing and/or other impacts on climate, locally or globally. See also: the IPCC Report on Land Use, Land-Use Change, and Forestry (IPCC, 2000).

\section{Last Interglacial (LIG)}

See Interglacial

\section{Learning by Doing}

As researchers and firms gain familiarity with a new technological process, or acquire experience through expanded production they can discover ways to improve processes and reduce cost. Learning by Doing is a type of experience-based technological change.

\section{Level of Scientific Understanding (LOSU)}

This is an index on a 5-step scale (high, medium, medium-low, low and very low) designed to characterise the degree of scientific understanding of the radiative forcing agents that affect climate change. For each agent, the index represents a subjective judgement about the evidence for the physi$\mathrm{cal} / \mathrm{chemical}$ mechanisms determining the forcing and the consensus surrounding the quantitative estimate and its uncertainty.

\section{Likelihood}

The likelihood of an occurrence, an outcome or a result, where this can be estimated probabilistically, is expressed in IPCC reports using a standard terminology defined as follows:

\begin{tabular}{|l|l|}
\hline Terminology & Likelihood of the occurrence / outcome \\
\hline Virtually certain & $>99 \%$ probability of occurrence \\
Very likely & $>90 \%$ probability \\
Likely & $>66 \%$ probability \\
More likely than not & $>50 \%$ probability \\
About as likely as not & 33 to $66 \%$ probability \\
Unlikely & $<33 \%$ probability \\
Very unlikely & $<10 \%$ probability \\
Exceptionally unlikely & $<1 \%$ probability \\
\hline
\end{tabular}

See also Confidence; Uncertainty 


\section{M.}

\section{Macroeconomic costs}

These costs are usually measured as changes in Gross Domestic Product or changes in the growth of Gross Domestic Product, or as loss of welfare or of consumption.

\section{Malaria}

Endemic or epidemic parasitic disease caused by species of the genus Plasmodium (Protozoa) and transmitted to humans by mosquitoes of the genus Anopheles; produces bouts of high fever and systemic disorders, affects about 300 million and kills approximately 2 million people worldwide every year.

\section{Market Exchange Rate (MER)}

This is the rate at which foreign currencies are exchanged. Most economies post such rates daily and they vary little across all the exchanges. For some developing economies official rates and black-market rates may differ significantly and the MER is difficult to pin down.

\section{Market impacts}

Impacts that can be quantified in monetary terms, and directly affect Gross Domestic Product - e.g. changes in the price of agricultural inputs and/or goods. See also Non-market impacts.

\section{Market potential}

See Mitigation potential.

\section{Mass balance (of glaciers, ice caps or ice sheets)}

The balance between the mass input to an ice body (accumulation) and the mass loss (ablation, iceberg calving). Mass balance terms include the following:

Specific mass balance: net mass loss or gain over a hydrological cycle at a point on the surface of a glacier.

Total mass balance (of the glacier): The specific mass balance spatially integrated over the entire glacier area; the total mass a glacier gains or loses over a hydrological cycle.

Mean specific mass balance: The total mass balance per unit area of the glacier. If surface is specified (specific surface mass balance, etc.) then ice-flow contributions are not considered; otherwise, mass balance includes contributions from ice flow and iceberg calving. The specific surface mass balance is positive in the accumulation area and negative in the ablation area.

\section{Mean Sea Level}

Mean sea level is normally defined as the average relative sea level over a period, such as a month or a year, long enough to average out transients such as waves and tides. Relative sea level is sea level measured by a tide gauge with respect to the land upon which it is situated.

See Sea level change/sea level rise.

\section{Measures}

Measures are technologies, processes, and practices that reduce greenhouse gas emissions or effects below anticipated future levels. Examples of measures are renewable energy technologies, waste minimisation processes, and public transport commuting practices, etc. See also Policies.

\section{Meridional Overturning Circulation (MOC)}

A zonally averaged, large scale meridional (north-south) overturning circulation in the oceans. In the Atlantic such a circulation transports relatively warm upper-ocean waters northward, and relatively cold deep waters southward. The Gulf Stream forms part of this Atlantic circulation.

\section{Methane $\left(\mathrm{CH}_{4}\right)$}

Methane is one of the six greenhouse gases to be mitigated under the Kyoto Protocol and is the major component of natural gas and associated with all hydrocarbon fuels, animal husbandry and agriculture. Coal-bed methane is the gas found in coal seams.

\section{Methane recovery}

Methane emissions, e.g. from oil or gas wells, coal beds, peat bogs, gas transmission pipelines, landfills, or anaerobic digesters, may be captured and used as a fuel or for some other economic purpose (e.g. chemical feedstock).

\section{Metric}

A consistent measurement of a characteristic of an object or activity that is otherwise difficult to quantify.

\section{Millennium Development Goals (MDGs)}

A set of time-bound and measurable goals for combating poverty, hunger, disease, illiteracy, discrimination against women and environmental degradation, agreed at the UN Millennium Summit in 2000.

\section{Mitigation}

Technological change and substitution that reduce resource inputs and emissions per unit of output. Although several social, economic and technological policies would produce an emission reduction, with respect to Climate Change, mitigation means implementing policies to reduce greenhouse gas emissions and enhance sinks.

\section{Mitigative capacity}

This is a country's ability to reduce anthropogenic greenhouse gas emissions or to enhance natural sinks, where ability refers to skills, competencies, fitness and proficiencies that a country has attained and depends on technology, institutions, wealth, equity, infrastructure and information. Mitigative capacity is rooted in a country's sustainable development path.

\section{Mitigation Potential}

In the context of climate change mitigation, the mitigation potential is the amount of mitigation that could be - but is not yet - realised over time.

Market potential is the mitigation potential based on private costs and private discount rates, which might be expected to occur under forecast market conditions, including policies and measures currently in place, noting that barriers limit actual uptake. Private costs and discount rates reflect the perspective of private consumers and companies.

Economic potential is the mitigation potential that takes into account social costs and benefits and social discount rates, assuming that market efficiency is improved by policies and measures and barriers are removed. Social costs and discount rates reflect the perspective of society. Social discount rates are lower than those used by private investors.

Studies of market potential can be used to inform policy makers about mitigation potential with existing policies and barriers, while studies of economic potential show what might be achieved if appropriate new and additional policies were put into place to remove barriers and include social costs and benefits. The economic potential is therefore generally greater than the market potential.

Technical potential is the amount by which it is possible to reduce greenhouse gas emissions or improve energy efficiency by implementing a technology or practice that has already been demonstrated. No explicit reference to costs is made but adopting 'practical constraints' may take implicit economic considerations into account.

\section{Model}

See Climate model; Bottom-up model; Top-down model

\section{Model hierarchy}

See Climate model

\section{Monsoon}

A monsoon is a tropical and subtropical seasonal reversal in both the surface winds and associated precipitation, caused by differential heating between a continental-scale land mass and the adjacent ocean. Monsoon rains occur mainly over land in summer. 


\section{Morbidity}

Rate of occurrence of disease or other health disorder within a population, taking account of the age-specific morbidity rates. Morbidity indicators include chronic disease incidence/ prevalence, rates of hospitalisation, primary care consultations, disability-days (i.e., days of absence from work), and prevalence of symptoms.

\section{Mortality}

Rate of occurrence of death within a population; calculation of mortality takes account of age-specific death rates, and can thus yield measures of life expectancy and the extent of premature death.

\section{N.}

\section{Net market benefits}

Climate change, especially moderate climate change, is expected to bring positive and negative impacts to market-based sectors, but with significant differences across different sectors and regions and depending on both the rate and magnitude of climate change. The sum of the positive and negative market-based benefits and costs summed across all sectors and all regions for a given period is called net market benefits. Net market benefits exclude any non-market impacts.

\section{Nitrous oxide $\left(\mathrm{N}_{2} \mathbf{0}\right)$}

One of the six types of greenhouse gases to be curbed under the Kyoto Protocol. The main anthropogenic source of nitrous oxide is agriculture (soil and animal manure management), but important contributions also come from sewage treatment, combustion of fossil fuel, and chemical industrial processes. Nitrous oxide is also produced naturally from a wide variety of biological sources in soil and water, particularly microbial action in wet tropical forests.

\section{Non-governmental Organisation (NGO)}

A non-profit group or association organised outside of institutionalised political structures to realise particular social and/or environmental objectives or serve particular constituencies. Source: http://www.edu.gov.nf.ca/ curriculum/teched/resources/glos-biodiversity.html

\section{Non-market impacts}

Impacts that affect ecosystems or human welfare, but that are not easily expressed in monetary terms, e.g., an increased risk of premature death, or increases in the number of people at risk of hunger. See also market impacts.

\section{O.}

\section{Ocean acidification}

A decrease in the $p H$ of sea water due to the uptake of anthropogenic carbon dioxide.

\section{Opportunities}

Circumstances to decrease the gap between the market potential of any technology or practice and the economic potential, or technical potential.

\section{Ozone $\left(\mathrm{O}_{3}\right)$}

Ozone, the tri-atomic form of oxygen, is a gaseous atmospheric constituent. In the troposphere, ozone is created both naturally and by photochemical reactions involving gases resulting from human activities (smog). Troposphere ozone acts as a greenhouse gas. In the stratosphere, ozone is created by the interaction between solar ultraviolet radiation and molecular oxygen $\left(\mathrm{O}_{2}\right)$. Stratospheric ozone plays a dominant role in the stratospheric radiative balance. Its concentration is highest in the ozone layer.

\section{P.}

\section{Paleoclimate}

Climate during periods prior to the development of measuring instruments, including historic and geologic time, for which only proxy climate records are available.

\section{Patterns of climate variability}

Natural variability of the climate system, in particular on seasonal and longer time scales, predominantly occurs with preferred spatial patterns and time scales, through the dynamical characteristics of the atmospheric circulation and through interactions with the land and ocean surfaces. Such patterns are often called regimes, modes or teleconnections. Examples are the North Atlantic Oscillation (NAO), the Pacific-North American pattern (PNA), the El Niño-Southern Oscillation (ENSO), the Northern Annular Mode (NAM; previously called Arctic Oscillation, AO) and the Southern Annular Mode (SAM; previously called the Antarctic Oscillation, AAO). Many of the prominent modes of climate variability are discussed in section 3.6 of the Working Group I Report.

\section{Percentile}

A percentile is a value on a scale of zero to one hundred that indicates the percentage of the data set values that is equal to or below it. The percentile is often used to estimate the extremes of a distribution. For example, the $90^{\text {th }}\left(10^{\text {th }}\right)$ percentile may be used to refer to the threshold for the upper (lower) extremes.

\section{Perfluorocarbons (PFCs)}

Among the six greenhouse gases to be abated under the Kyoto Protocol. These are by-products of aluminium smelting and uranium enrichment. They also replace chlorofluorocarbons in manufacturing semiconductors.

\section{Permafrost}

Ground (soil or rock and included ice and organic material) that remains at or below $0^{\circ} \mathrm{C}$ for at least two consecutive years (Van Everdingen, 1998). See also Frozen ground.

\section{$\mathrm{pH}$}

$\mathrm{pH}$ is a dimensionless measure of the acidity of water (or any solution). Pure water has a $\mathrm{pH}=7$. Acid solutions have a $\mathrm{pH}$ smaller than 7 and basic solutions have a $\mathrm{pH}$ larger than 7. $\mathrm{pH}$ is measured on a logarithmic scale. Thus, a $\mathrm{pH}$ decrease of 1 unit corresponds to a 10 -fold increase in the acidity.

\section{Phenology}

The study of natural phenomena in biological systems that recur periodically (e.g., development stages, migration) and their relation to climate and seasonal changes.

\section{Photosynthesis}

The process by which green plants, algae and some bacteria take carbon dioxide from the air (or bicarbonate in water) to build carbohydrates. There are several pathways of photosynthesis with different responses to atmospheric carbon dioxide concentrations. See Carbon dioxide fertilisation.

\section{Plankton}

Micro-organisms living in the upper layers of aquatic systems. A distinction is made between phytoplankton, which depend on photosynthesis for their energy supply, and zooplankton, which feed on phytoplankton.

\section{Policies}

In United Nations Framework Convention on Climate Change (UNFCCC) parlance, policies are taken and/or mandated by a government - often in conjunction with business and industry within its own country, or with other countries - to accelerate mitigation and adaptation measures. Examples of policies are carbon or other energy taxes, fuel efficiency standards for automobiles, etc. Common and co-ordinated or harmonised policies refer to those adopted jointly by parties. See also Measures.

\section{Portfolio}

A coherent set of a variety of measures and/or technologies that policy makers can use to achieve a postulated policy target. By widening the scope in measures and technologies more diverse events and uncertainties can be addressed. 


\section{Post-SRES (scenarios)}

Baseline and mitigation emission scenarios published after completion of the IPCC Special Report on Emission Scenarios (SRES) (Nakičenovič and Swart, 2000), i.e. after the year 2000.

\section{Pre-industrial}

See Industrial revolution.

\section{Projection}

A potential future evolution of a quantity or set of quantities, often computed with the aid of a model. Projections are distinguished from predictions in order to emphasise that projections involve assumptions concerning, for example, future socio-economic and technological developments that may or may not be realised, and are therefore subject to substantial uncertainty. See also Climate projection; Climate prediction.

\section{Purchasing Power Parity (PPP)}

The purchasing power of a currency is expressed using a basket of goods and services that can be bought with a given amount in the home country. International comparison of e.g. Gross Domestic Products (GDP) of countries can be based on the purchasing power of currencies rather than on current exchange rates. PPP estimates tend to lower per capita GDPs in industrialised countries and raise per capita GDPs in developing countries.

\section{R.}

\section{Radiative forcing}

Radiative forcing is the change in the net, downward minus upward, irradiance (expressed in Watts per square metre, $\mathrm{W} / \mathrm{m}^{2}$ ) at the tropopause due to a change in an external driver of climate change, such as, for example, a change in the concentration of carbon dioxide or the output of the Sun. Radiative forcing is computed with all tropospheric properties held fixed at their unperturbed values, and after allowing for stratospheric temperatures, if perturbed, to readjust to radiative-dynamical equilibrium. Radiative forcing is called instantaneous if no change in stratospheric temperature is accounted for. For the purposes of this report, radiative forcing is further defined as the change relative to the year 1750 and, unless otherwise noted, refers to a global and annual average value.

\section{Reforestation}

Planting of forests on lands that have previously contained forests but that have been converted to some other use. For a discussion of the term forest and related terms such as afforestation, reforestation and deforestation, see the IPCC Report on Land Use, Land-Use Change and Forestry (IPCC, 2000). See also the Report on Definitions and Methodological Options to Inventory Emissions from Direct Human-induced Degradation of Forests and Devegetation of Other Vegetation Types (IPCC, 2003)

\section{Region}

A region is a territory characterised by specific geographical and climatological features. The climate of a region is affected by regional and local scale forcings like topography, land-use characteristics, lakes etc., as well as remote influences from other regions.

\section{Resilience}

The ability of a social or ecological system to absorb disturbances while retaining the same basic structure and ways of functioning, the capacity for self-organisation, and the capacity to adapt to stress and change.

\section{Retrofitting}

Retrofitting means to install new or modified parts or equipment, or undertake structural modifications, to existing infrastructure that were either not available or not considered necessary at the time of construction. The purpose of retrofitting in the context of climate change is generally to ensure that existing infrastructure meets new design specifications that may be required under altered climate conditions.

\section{Runoff}

That part of precipitation that does not evaporate and is not transpired, but flows over the ground surface and returns to bodies of water. See Hydrological cycle

\section{S.}

\section{Salinisation}

The accumulation of salts in soils.

\section{Saltwater intrusion}

Displacement of fresh surface water or groundwater by the advance of saltwater due to its greater density. This usually occurs in coastal and estuarine areas due to reducing land-based influence (e.g., either from reduced runoff and associated groundwater recharge, or from excessive water withdrawals from aquifers) or increasing marine influence (e.g., relative sea-level rise).

\section{Scenario}

A plausible and often simplified description of how the future may develop, based on a coherent and internally consistent set of assumptions about driving forces and key relationships. Scenarios may be derived from projections, but are often based on additional information from other sources, sometimes combined with a narrative storyline. See also SRES scenarios; Climate scenario; Emission scenarios.

\section{Sea-ice biome}

The biome formed by all marine organisms living within or on the floating sea ice (frozen seawater) of the polar oceans.

\section{Sea ice}

Any form of ice found at sea that has originated from the freezing of sea water. Sea ice may be discontinuous pieces (ice floes) moved on the ocean surface by wind and currents (pack ice), or a motionless sheet attached to the coast (land-fast ice). Sea ice less than one year old is called first-year ice. Multi-year ice is sea ice that has survived at least one summer melt season.

\section{Sea level change/sea level rise}

Sea level can change, both globally and locally, due to (i) changes in the shape of the ocean basins, (ii) changes in the total mass of water and (iii) changes in water density. Factors leading to sea level rise under global warming include both increases in the total mass of water from the melting of land-based snow and ice, and changes in water density from an increase in ocean water temperatures and salinity changes. Relative sea level rise occurs where there is a local increase in the level of the ocean relative to the land, which might be due to ocean rise and/or land level subsidence. See also Mean Sea Level, Thermal expansion.

\section{Seasonally frozen ground}

See Frozen ground

\section{Sensitivity}

Sensitivity is the degree to which a system is affected, either adversely or beneficially, by climate variability or climate change. The effect may be direct (e.g., a change in crop yield in response to a change in the mean, range, or variability of temperature) or indirect (e.g., damages caused by an increase in the frequency of coastal flooding due to sea level rise).

This concept of sensitivity is not to be confused with climate sensitivity, which is defined separately above.

\section{Singularity}

A trait marking one phenomenon or aspect as distinct from others; something singular, distinct, peculiar, uncommon or unusual.

\section{Sink}

Any process, activity or mechanism which removes a greenhouse gas, an aerosol or a precursor of a greenhouse gas or aerosol from the atmosphere. 


\section{Snow pack}

A seasonal accumulation of slow-melting snow.

\section{Soil temperature}

The temperature of the ground near the surface (often within the first $10 \mathrm{~cm})$.

\section{Solar activity}

The Sun exhibits periods of high activity observed in numbers of sunspots, as well as radiative output, magnetic activity, and emission of high energy particles. These variations take place on a range of time-scales from millions of years to minutes

\section{Solar radiation}

Electromagnetic radiation emitted by the Sun. It is also referred to as shortwave radiation. Solar radiation has a distinctive range of wavelengths (spectrum) determined by the temperature of the Sun, peaking in visible wavelengths. See also Thermal infrared radiation, Total Solar Irradiance

\section{Source}

Source mostly refers to any process, activity or mechanism that releases a greenhouse gas, an aerosol, or a precursor of a greenhouse gas or aerosol into the atmosphere. Source can also refer to e.g. an energy source.

\section{Spatial and temporal scales}

Climate may vary on a large range of spatial and temporal scales. Spatial scales may range from local (less than $100,000 \mathrm{~km}^{2}$ ), through regional $\left(100,000\right.$ to 10 million $\left.\mathrm{km}^{2}\right)$ to continental $\left(10\right.$ to 100 million $\left.\mathrm{km}^{2}\right)$. Temporal scales may range from seasonal to geological (up to hundreds of millions of years).

\section{SRES scenarios}

SRES scenarios are emission scenarios developed by Nakičenovič and Swart (2000) and used, among others, as a basis for some of the climate projections used in the Fourth Assessment Report. The following terms are relevant for a better understanding of the structure and use of the set of SRES scenarios:

Scenario Family: Scenarios that have a similar demographic, societal, economic and technical-change storyline. Four scenario families comprise the SRES scenario set: A1, A2, B1 and B2.

Illustrative Scenario: A scenario that is illustrative for each of the six scenario groups reflected in the Summary for Policymakers of Nakičenovič et al. (2000). They include four revised 'scenario markers' for the scenario groups A1B, A2, B1, B2, and two additional scenarios for the A1FI and A1T groups. All scenario groups are equally sound.

Marker Scenario: A scenario that was originally posted in draft form on the SRES website to represent a given scenario family. The choice of markers was based on which of the initial quantifications best reflected the storyline, and the features of specific models. Markers are no more likely than other scenarios, but are considered by the SRES writing team as illustrative of a particular storyline. They are included in revised form in Nakičenovič and Swart (2000). These scenarios received the closest scrutiny of the entire writing team and via the SRES open process. Scenarios were also selected to illustrate the other two scenario groups.

Storyline: A narrative description of a scenario (or family of scenarios), highlighting the main scenario characteristics, relationships between key driving forces and the dynamics of their evolution.

\section{Stabilisation}

Keeping constant the atmospheric concentrations of one or more greenhouse gases (e.g. carbon dioxide) or of a CO2-equivalent basket of greenhouse gases. Stabilisation analyses or scenarios address the stabilisation of the concentration of greenhouse gases in the atmosphere.

\section{Stakeholder}

A person or an organisation that has a legitimate interest in a project or entity, or would be affected by a particular action or policy.

\section{Standards}

Set of rules or codes mandating or defining product performance (e.g., grades, dimensions, characteristics, test methods, and rules for use). Product, technology or performance standards establish minimum requirements for affected products or technologies. Standards impose reductions in greenhouse gas emissions associated with the manufacture or use of the products and/or application of the technology.

\section{Storm surge}

The temporary increase, at a particular locality, in the height of the sea due to extreme meteorological conditions (low atmospheric pressure and/or strong winds). The storm surge is defined as being the excess above the level expected from the tidal variation alone at that time and place.

\section{Storm tracks}

Originally, a term referring to the tracks of individual cyclonic weather systems, but now often generalised to refer to the regions where the main tracks of extratropical disturbances occur as sequences of low (cyclonic) and high (anticyclonic) pressure systems.

\section{Stratosphere}

The highly stratified region of the atmosphere above the troposphere extending from about $10 \mathrm{~km}$ (ranging from $9 \mathrm{~km}$ in high latitudes to $16 \mathrm{~km}$ in the tropics on average) to about $50 \mathrm{~km}$ altitude.

\section{Streamflow}

Water flow within a river channel, for example expressed in $\mathrm{m}^{3} / \mathrm{s}$. A synonym for river discharge.

\section{Structural change}

Changes, for example, in the relative share of Gross Domestic Product produced by the industrial, agricultural, or services sectors of an economy; or more generally, systems transformations whereby some components are either replaced or potentially substituted by other ones.

\section{Sulphurhexafluoride $\left(\mathbf{S F}_{6}\right)$}

One of the six greenhouse gases to be curbed under the Kyoto Protocol. It is largely used in heavy industry to insulate high-voltage equipment and to assist in the manufacturing of cable-cooling systems and semi-conductors.

\section{Surface temperature}

See Global surface temperature.

\section{Sustainable Development (SD)}

The concept of sustainable development was introduced in the World Conservation Strategy (IUCN 1980) and had its roots in the concept of a sustainable society and in the management of renewable resources. Adopted by the WCED in 1987 and by the Rio Conference in 1992 as a process of change in which the exploitation of resources, the direction of investments, the orientation of technological development, and institutional change are all in harmony and enhance both current and future potential to meet human needs and aspirations. SD integrates the political, social, economic and environmental dimensions.

\section{T.}

\section{Tax}

A carbon tax is a levy on the carbon content of fossil fuels. Because virtually all of the carbon in fossil fuels is ultimately emitted as carbon diox$i d e$, a carbon tax is equivalent to an emission tax on each unit of $\mathrm{CO} 2$ equivalent emissions. An energy tax - a levy on the energy content of fuels - reduces demand for energy and so reduces carbon dioxide emissions from fossil fuel use. An eco-tax is designed to influence human behaviour (specifically economic behaviour) to follow an ecologically benign path. An international carbon/emission/energy tax is a tax imposed on specified sources in participating countries by an international agreement. A harmonised tax commits participating countries to impose a tax at a common rate on the same sources. A tax credit is a reduction of tax in order to stimulate purchasing of or investment in a certain product, like GHG emission reducing technologies. A carbon charge is the same as a carbon tax. 


\section{Technological change}

Mostly considered as technological improvement, i.e. more or better goods and services can be provided from a given amount of resources (production factors). Economic models distinguish autonomous (exogenous), endogenous and induced technological change. Autonomous (exogenous) technological change is imposed from outside the model, usually in the form of a time trend affecting energy demand or world output growth. Endogenous technological change is the outcome of economic activity within the model, i.e. the choice of technologies is included within the model and affects energy demand and/or economic growth. Induced technological change implies endogenous technological change but adds further changes induced by policies and measures, such as carbon taxes triggering $R \& D$ efforts.

\section{Technology}

The practical application of knowledge to achieve particular tasks that employs both technical artefacts (hardware, equipment) and (social) information ('software', know-how for production and use of artefacts).

\section{Technology transfer}

The exchange of knowledge, hardware and associated software, money and goods among stakeholders that leads to the spreading of technology for adaptation or mitigation The term encompasses both diffusion of technologies and technological cooperation across and within countries.

\section{Thermal expansion}

In connection with sea-level rise, this refers to the increase in volume (and decrease in density) that results from warming water. A warming of the ocean leads to an expansion of the ocean volume and hence an increase in sea level. See Sea level change.

\section{Thermal infrared radiation}

Radiation emitted by the Earth's surface, the atmosphere and the clouds. It is also known as terrestrial or longwave radiation, and is to be distinguished from the near-infrared radiation that is part of the solar spectrum. Infrared radiation, in general, has a distinctive range of wavelengths (spectrum) longer than the wavelength of the red colour in the visible part of the spectrum. The spectrum of thermal infrared radiation is practically distinct from that of shortwave or solar radiation because of the difference in temperature between the Sun and the Earth-atmosphere system.

\section{Tide gauge}

A device at a coastal location (and some deep sea locations) that continuously measures the level of the sea with respect to the adjacent land. Time averaging of the sea level so recorded gives the observed secular changes of the relative sea level. See Sea level change/sea level rise.

\section{Top-down models}

Top-down model apply macroeconomic theory, econometric and optimization techniques to aggregate economic variables. Using historical data on consumption, prices, incomes, and factor costs, top-down models assess final demand for goods and services, and supply from main sectors, like the energy sector, transportation, agriculture, and industry. Some topdown models incorporate technology data, narrowing the gap to bottomup models.

\section{Total Solar Irradiance (TSI)}

The amount of solar radiation received outside the Earth's atmosphere on a surface normal to the incident radiation, and at the Earth's mean distance from the sun. Reliable measurements of solar radiation can only be made from space and the precise record extends back only to 1978. The generally accepted value is 1,368 Watts per square meter $\left(\mathrm{W} \mathrm{m}^{-2}\right)$ with an accuracy of about $0.2 \%$. Variations of a few tenths of a percent are common, usually associated with the passage of sunspots across the solar disk. The solar cycle variation of TSI is on the order of $0.1 \%$. Source: AMS, 2000.

\section{Tradable permit}

A tradable permit is an economic policy instrument under which rights to discharge pollution - in this case an amount of greenhouse gas emissions
- can be exchanged through either a free or a controlled permit-market. An emission permit is a non-transferable or tradable entitlement allocated by a government to a legal entity (company or other emitter) to emit a specified amount of a substance.

\section{Tropopause}

The boundary between the troposphere and the stratosphere.

\section{Troposphere}

The lowest part of the atmosphere from the surface to about $10 \mathrm{~km}$ in altitude in mid-latitudes (ranging from $9 \mathrm{~km}$ in high latitudes to $16 \mathrm{~km}$ in the tropics on average), where clouds and weather phenomena occur. In the troposphere, temperatures generally decrease with height.

\section{U.}

\section{Uncertainty}

An expression of the degree to which a value (e.g., the future state of the climate system) is unknown. Uncertainty can result from lack of information or from disagreement about what is known or even knowable. It may have many types of sources, from quantifiable errors in the data to ambiguously defined concepts or terminology, or uncertain projections of human behaviour. Uncertainty can therefore be represented by quantitative measures, for example, a range of values calculated by various models, or by qualitative statements, for example, reflecting the judgement of a team of experts (see Moss and Schneider, 2000; Manning et al., 2004). See also Likelihood; Confidence.

\section{United Nations Framework Convention on Climate Change (UNFCCC)}

The Convention was adopted on 9 May 1992 in New York and signed at the 1992 Earth Summit in Rio de Janeiro by more than 150 countries and the European Community. Its ultimate objective is the "stabilisation of greenhouse gas concentrations in the atmosphere at a level that would prevent dangerous anthropogenic interference with the climate system". It contains commitments for all Parties. Under the Convention, Parties included in Annex I (all OECD member countries in the year 1990 and countries with economies in transition) aim to return greenhouse gas emissions not controlled by the Montreal Protocol to 1990 levels by the year 2000. The Convention entered in force in March 1994. See Kyoto Protocol.

\section{Uptake}

The addition of a substance of concern to a reservoir. The uptake of carbon containing substances, in particular carbon dioxide, is often called (carbon) sequestration.

\section{Urbanisation}

The conversion of land from a natural state or managed natural state (such as agriculture) to cities; a process driven by net rural-to-urban migration through which an increasing percentage of the population in any nation or region come to live in settlements that are defined as urban centres.

\section{V.}

\section{Vector}

An organism, such as an insect, that transmits a pathogen from one host to another.

\section{Voluntary action}

Informal programmes, self-commitments and declarations, where the parties (individual companies or groups of companies) entering into the action set their own targets and often do their own monitoring and reporting.

\section{Voluntary agreement}

An agreement between a government authority and one or more private parties to achieve environmental objectives or to improve environmental performance beyond compliance to regulated obligations. Not all voluntary agreements are truly voluntary; some include rewards and/or penalties associated with joining or achieving commitments. 


\section{Vulnerability}

Vulnerability is the degree to which a system is susceptible to, and unable to cope with, adverse effects of climate change, including climate variability and extremes. Vulnerability is a function of the character, magnitude, and rate of climate change and variation to which a system is exposed, its sensitivity, and its adaptive capacity.

\section{W.}

\section{Water consumption}

Amount of extracted water irretrievably lost during its use (by evaporation and goods production). Water consumption is equal to water withdrawal minus return flow.

\section{Water stress}

A country is water stressed if the available freshwater supply relative to water withdrawals acts as an important constraint on development. In global-scale assessments, basins with water stress are often defined as having a per capita water availability below $1,000 \mathrm{~m}^{3} / \mathrm{yr}$ (based on long-term average runoff). Withdrawals exceeding $20 \%$ of renewable water supply have also been used as an indicator of water stress. A crop is water stressed if soil available water, and thus actual evapotranspiration, is less than potential evapotranspiration demands.

\section{Z.}

\section{Zooplankton}

See Plankton

\section{References}

Glossaries of the contributions of Working Groups I, II and III to the IPCC Fourth Assessment Report.

AMS, 2000: AMS Glossary of Meteorology, 2nd Ed. American Meteorological Society, Boston, MA, http://amsglossary.allenpress.com/glossary/browse.

Cleveland C.J. and C. Morris, 2006: Dictionary of Energy, Elsevier, Amsterdam, 502p

Heim, R.R., 2002: A Review of Twentieth-Century Drought Indices Used in the United States. Bull. Am. Meteorol. Soc., 83, 1149-1165

IPCC, 1996: Climate Change 1995: The Science of Climate Change. Contribution of Working Group I to the Second Assessment Report of the Intergovernmental Panel on Climate Change [Houghton., J.T., et al. (eds.)]. Cambridge University Press, Cambridge, United Kingdom and New York, NY, USA, 572 pp.

IPCC, 2000: Land Use, Land-Use Change, and Forestry. Special Report of the Intergovernmental Panel on Climate Change [Watson, R.T., et al. (eds.)]. Cambridge University Press, Cambridge, United Kingdom and New York, NY, USA, 377 pp.

IPCC, 2003: Definitions and Methodological Options to Inventory Emissions from Direct Human-Induced Degradation of Forests and Devegetation of Other Vegetation Types [Penman, J., et al. (eds.)]. The Institute for Global Environmental Strategies (IGES), Japan , 32 pp.

IUCN, 1980: The World Conservation Strategy: living resource conservation for sustainable development, Gland, Switzerland, IUCN/UNEP/ WWF.

Manning, M., et al., 2004: IPCC Workshop on Describing Scientific Uncertainties in Climate Change to Support Analysis of Risk of Options. Workshop Report. Intergovernmental Panel on Climate Change, Geneva.

Moss, R., and S. Schneider, 2000: Uncertainties in the IPCC TAR: Recommendations to Lead Authors for More Consistent Assessment and Reporting. In: IPCC Supporting Material: Guidance Papers on Cross Cutting Issues in the Third Assessment Report of the IPCC. [Pachauri, R., T. Taniguchi, and K. Tanaka (eds.)]. Intergovernmental Panel on Climate Change, Geneva, pp. 33-51.

Nakičenovič, N., and R. Swart (eds.), 2000: Special Report on Emissions Scenarios. A Special Report of Working Group III of the Intergovernmental Panel on Climate Change. Cambridge University Press, Cambridge, United Kingdom and New York, NY, USA, 599 pp.

Van Everdingen, R. (ed.): 1998. Multi-Language Glossary of Permafrost and Related Ground-Ice Terms, revised May 2005. National Snow and Ice Data Center/World Data Center for Glaciology, Boulder, CO, http://nsidc.org/fgdc/glossary/. 


\section{Acronyms, chemical symbols; scientific units; country groupings}

\section{III.1 Acronyms and chemical symbols}

A1 A family of scenarios in the IPCC Special Report on Emission Scenarios; see glossary under SRES scenarios

A1T One of the six SRES marker scenarios; see glossary under SRES scenarios

A1B One of the six SRES marker scenarios; see glossary under SRES scenarios

A1FI One of the six SRES marker scenarios; see glossary under SRES scenarios

A2 A family of scenarios in the IPCC Special Report on Emission Scenarios; also one of the six SRES marker scenarios; see glossary under SRES scenarios

AOGCM Atmosphere-Ocean General Circulation Model; see glossary under climate model

B1 A family of scenarios in the IPCC Special Report on Emission Scenarios; also denotes one of the six SRES marker scenarios; see glossary under SRES scenarios

B2 A family of scenarios in the IPCC Special Report on Emission Scenarios; also denotes one of the six SRES marker scenarios; see glossary under SRES scenarios

$\mathrm{CH}_{4} \quad$ Methane; see glossary

$\mathrm{CFC} \quad$ Chlorofluorocarbon; see glossary

$\mathrm{CO}_{2} \quad$ Carbon dioxide; see glossary

EIT Economies in transition; see glossary
EMIC Earth Model of Intermediate Complexity

ENSO El Niño-Southern Oscillation; see glossary

F-Gases Fluorinated gases covered under the Kyoto Protocol; see glossary under F-Gases

GDP Gross Domestic Product; see glossary

HCFC Hydrochlorofluorocarbon; see glossary

HFC Hydrofluorocarbon; see glossary

LOSU Level of scientific understanding; see glossary

MOC Meridional overturning circulation; see glossary

$\mathrm{N}_{2} \mathrm{O} \quad$ Nitrous oxide; see glossary

OECD Organisation for Economic Cooperation and Development; see www.oecd.org

PFC Perfluorocarbon; see glossary

$\mathrm{pH} \quad$ See glossary under $\mathrm{pH}$

PPP Purchasing Power Parity; see glossary

RD\&D Research, development and demonstration

SCM Simple Climate Model

SF $\quad$ Sulfur hexafluoride; see glossary

SRES Special Report on Emission Scenarios; see glossary under SRES scenarios

UNFCCC United Nations Framework Convention on Climate Change; see www.unfccc.int

\section{III.2 Scientific units}

\begin{tabular}{|c|c|c|c|c|c|}
\hline \multicolumn{6}{|c|}{ SI (Système Internationale) units } \\
\hline \multicolumn{2}{|c|}{ Physical Quantity } & \multicolumn{2}{|l|}{ Name of Unit } & \multicolumn{2}{|l|}{ Symbol } \\
\hline \multicolumn{2}{|l|}{ length } & \multicolumn{2}{|l|}{ metre } & \multicolumn{2}{|l|}{$\mathrm{m}$} \\
\hline \multicolumn{2}{|l|}{ mass } & \multicolumn{2}{|l|}{ kilogram } & \multicolumn{2}{|l|}{$\mathrm{kg}$} \\
\hline \multicolumn{2}{|l|}{ time } & \multicolumn{2}{|l|}{ second } & \multicolumn{2}{|l|}{$\mathrm{s}$} \\
\hline \multicolumn{2}{|c|}{ thermodynamic temperature } & \multicolumn{2}{|l|}{ kelvin } & \multicolumn{2}{|l|}{$\mathrm{K}$} \\
\hline \multicolumn{6}{|c|}{ Fractions and multiples } \\
\hline \multicolumn{2}{|c|}{ Fraction $\quad$ Prefix } & Symbol & Multiple & Prefix & Symbol \\
\hline $10^{-1}$ & deci & $\mathrm{d}$ & 10 & deca & da \\
\hline $10^{-2}$ & centi & $\mathrm{c}$ & $10^{2}$ & hecto & $\mathrm{h}$ \\
\hline $10^{-3}$ & milli & $\mathrm{m}$ & $10^{3}$ & kilo & $\mathrm{k}$ \\
\hline $10^{-6}$ & micro & $\mu$ & $10^{6}$ & mega & M \\
\hline $10^{-9}$ & nano & n & $10^{9}$ & giga & $\mathrm{G}$ \\
\hline $10^{-12}$ & pico & $\mathrm{p}$ & $10^{12}$ & tera & $\mathrm{T}$ \\
\hline $10^{-15}$ & femto & $\mathrm{f}$ & $10^{15}$ & peta & $\mathrm{P}$ \\
\hline \multicolumn{6}{|c|}{ Non-SI units, quantities and related abbreviations } \\
\hline${ }^{\circ} \mathrm{C}$ & \multicolumn{5}{|c|}{ degree Celsius $\left(0^{\circ} \mathrm{C}=273 \mathrm{~K}\right.$ approximately $)$; temperature differences are also given } \\
\hline ppm & \multicolumn{5}{|c|}{ mixing ratio (as concentration measure of GHGs): parts per million $\left(10^{6}\right)$ by volume } \\
\hline $\mathrm{ppb}$ & \multicolumn{5}{|c|}{ mixing ratio (as concentration measure of GHGs): parts per billion $\left(10^{9}\right)$ by volume } \\
\hline ppt & \multicolumn{5}{|c|}{ mixing ratio (as concentration measure of GHGs): parts per trillion $\left(10^{12}\right)$ by volume } \\
\hline watt & \multicolumn{5}{|c|}{ power or radiant flux; 1 watt $=1$ Joule $/$ second $=1 \mathrm{~kg} \mathrm{~m}^{2} / \mathrm{s}^{3}$} \\
\hline $\mathrm{yr}$ & \multicolumn{5}{|l|}{ year } \\
\hline ky & \multicolumn{5}{|l|}{ thousands of years } \\
\hline bp & \multicolumn{5}{|l|}{ before present } \\
\hline $\mathrm{GtC}$ & \multicolumn{5}{|c|}{ gigatonnes (metric) of carbon } \\
\hline $\mathrm{GtCO}_{2}$ & gigatonnes (metric) & de $(1 \mathrm{GtC}=3$ & & & \\
\hline $\mathrm{CO}_{2}-\mathrm{eq}$ & $\begin{array}{l}\text { carbon dioxide-equi } \\
\text { of GHGs; see Box " }\end{array}$ & $\begin{array}{l}\text { measure for the } \\
\text {-equivalent em }\end{array}$ & $\begin{array}{l}\text { n (generall } \\
\text { nd concent }\end{array}$ & $\begin{array}{l}\text { q) or cor } \\
\text { pic } 2 \text { for }\end{array}$ & ally in ppm $\mathrm{CO}_{2}$-eq) \\
\hline
\end{tabular}




\section{III.3 Country groupings}

For the full set of countries belonging to UNFCCC Annex I, non-Annex I, and OECD, see http://www.unfccc.int and http://www.oecd.org.

Where relevant in this report, countries have been grouped into regions according to the classification of the UNFCCC and its Kyoto Protocol. Countries that have joined the European Union since 1997 are therefore still listed under EIT Annex I. The countries in each of the regional groupings employed in this report include:*

- EIT Annex I: Belarus, Bulgaria, Croatia, Czech Republic, Estonia, Hungary, Latvia, Lithuania, Poland, Romania, Russian Federation, Slovakia, Slovenia, Ukraine

- Europe Annex II \& M\&T: Austria, Belgium, Denmark, Finland, France, Germany, Greece, Iceland, Ireland, Italy, Liechtenstein, Luxembourg, Netherlands, Norway, Portugal, Spain, Sweden, Switzerland, United Kingdom; Monaco and Turkey

- JANZ: Japan, Australia, New Zealand.

- Middle East: Bahrain, Islamic Republic of Iran, Israel, Jordan, Kuwait, Lebanon, Oman, Qatar, Saudi Arabia, Syria, United Arab Emirates, Yemen

- Latin America \& the Caribbean: Antigua \& Barbuda, Argentina, Bahamas, Barbados, Belize, Bolivia, Brazil, Chile, Colombia, Costa Rica, Cuba, Dominica, Dominican Republic, Ecuador, El Salvador, Grenada, Guatemala, Guyana, Haiti, Honduras, Jamaica, Mexico, Nicaragua, Panama, Paraguay, Peru, Saint Lucia, St. Kitts-
Nevis-Anguilla, St. Vincent-Grenadines, Suriname, Trinidad and Tobago, Uruguay, Venezuela

- Non-Annex I East Asia: Cambodia, China, Korea (DPR), Laos (PDR), Mongolia, Republic of Korea, Viet Nam.

- South Asia: Afghanistan, Bangladesh, Bhutan, Comoros, Cook Islands, Fiji, India, Indonesia, Kiribati, Malaysia, Maldives, Marshall Islands, Micronesia (Federated States of), Myanmar, Nauru, Niue, Nepal, Pakistan, Palau, Papua New Guinea, Philippine, Samoa, Singapore, Solomon Islands, Sri Lanka, Thailand, Timor-L'Este, Tonga, Tuvalu, Vanuatu

- North America: Canada, United States of America.

- Other non-Annex I: Albania, Armenia, Azerbaijan, Bosnia Herzegovina, Cyprus, Georgia, Kazakhstan, Kyrgyzstan, Malta, Moldova, San Marino, Serbia, Tajikistan, Turkmenistan, Uzbekistan, Republic of Macedonia

- Africa: Algeria, Angola, Benin, Botswana, Burkina Faso, Burundi, Cameroon, Cape Verde, Central African Republic, Chad, Congo, Democratic Republic of Congo, Côte d'Ivoire, Djibouti, Egypt, Equatorial Guinea, Eritrea, Ethiopia, Gabon, Gambia, Ghana, Guinea, Guinea-Bissau, Kenya, Lesotho, Liberia, Libya, Madagascar, Malawi, Mali, Mauritania, Mauritius, Morocco, Mozambique, Namibia, Niger, Nigeria, Rwanda, Sao Tome and Principe, Senegal, Seychelles, Sierra Leone, South Africa, Sudan, Swaziland, Togo, Tunisia, Uganda, United Republic of Tanzania, Zambia, Zimbabwe

*A full set of data for all countries for 2004 for all regions was not available. 


\section{Annex IV}

\section{List of authors}

If country/countries of residence is/are different from nationality, nationality is mentioned last.

\section{IV.1 Core Writing Team members}

BERNSTEIN, Lenny

L.S. Bernstein \& Associates, L.L.C.

USA

BOSCH, Peter

IPCC WGIII TSU, Ecofys Netherlands, and Netherlands

Environmental Assessment Agency

THE NETHERLANDS

CANZIANI, Osvaldo

IPCC WGII Co-chair, Buenos Aires

ARGENTINA

CHEN, Zhenlin

Dept. of International Cooperation, China Meteorological

Administration

CHINA

CHRIST, Renate

Secretariat, Intergovernmental Panel on Climate Change (IPCC)

SWITZERLAND/AUSTRIA

DAVIDSON, Ogunlade

IPCC WGIII Co-chair, Faculty of Engineering, University of

Sierra Leone

SIERRA LEONE

HARE, William

Potsdam Institute for Climate Impact Research

GERMANY/AUSTRALIA

HUQ, Saleemul

International Institute for Environment and Development

(IIED)

UK/BANGLADESH

KAROLY, David

School of Meteorology, University of Oklahoma, USA, and

University of Melbourne, Australia

USA/AUSTRALIA

KATTSOV, Vladimir

Voeikov Main Geophysical Observatory

RUSSIA

KUNDZEWICZ, Zbyszek

Research Centre for Agricultural \& Forest Environment, Polish Academy of Sciences

POLAND
LIU, Jian

Secretariat, Intergovernmental Panel on Climate Change (IPCC) SWITZERLAND/CHINA

LOHMANN, Ulrike

ETH Zurich, Institute for Atmospheric and Climate Science SWITZERLAND

MANNING, Martin

IPCC WGI TSU, University Corporation for Atmospheric

Research

USA/NEW ZEALAND

MATSUNO, Taroh

Frontier Research Center for Global Change

Japan Agency for Marine-Earth Science and Technology

JAPAN

MENNE, Bettina

World Health Organization (WHO), Regional Office for

Europe

ITALY/GERMANY

METZ, Bert

IPCC WGIII Co-chair, Global Environmental Assessment Division, Netherlands Environmental Assessment Agency THE NETHERLANDS

MIRZA, Monirul

Adaptation \& Impacts Research Division (AIRD), Environment Canada, and Department of Physical and Environmental Sciences, University of Toronto

CANADA/BANGLADESH

NICHOLLS, Neville

School of Geography \& Environmental Science, Monash

University

AUSTRALIA

NURSE, Leonard

Barbados Centre for Resource Management and Environmental

Studies, University of West Indies

BARBADOS

PACHAURI, Rajendra

Chairman, Intergovernmental Panel on Climate Change

(IPCC) and Director-General, The Energy and Resources

Institute (TERI)

INDIA 
PALUTIKOF, Jean

IPCC WGII TSU, Met Office Hadley Centre

$\mathrm{UK}$

PARRY, Martin

IPCC WGII Co-chair, Met Office Hadley Centre, and Centre for Environmental Policy, Imperial College, University of London

UK

QIN, Dahe

IPCC WGI Co-chair, China Meteorological Administration CHINA

RAVINDRANATH, Nijavalli

Centre for Ecological Sciences, Indian Institute of Science INDIA

REISINGER, Andy

IPCC SYR TSU, Met Office Hadley Centre, UK, and The

Energy and Resources Institute (TERI), India

UK/INDIA/GERMANY

REN, Jiawen

Cold and Arid Regions Environment and Engineering Research Institute, Chinese Academy of Sciences

CHINA

RIAHI, Keywan

International Institute for Applied Systems Analysis (IIASA), and Graz University of Technology

AUSTRIA

ROSENZWEIG, Cynthia

Goddard Institute for Space Studies, National Aeronautics and Space Administration (NASA)

USA

RUSTICUCCI, Matilde

Departamento de Ciencias de la Atmósfera y los Océanos, Universidad de Buenos Aires

ARGENTINA
SCHNEIDER, Stephen

Department of Biological Sciences, Stanford University USA

SOKONA, Youba

Sahara and Sahel Observatory (OSS)

TUNISIA/MALI

SOLOMON, Susan

IPCC WGI Co-chair, NOAA Earth System Research

Laboratory

USA

STOTT, Peter

Met Office Hadley Centre

UK

STOUFFER, Ronald

NOAA Geophysical Fluid Dynamics Laboratory

USA

SUGIYAMA, Taishi

Climate Policy Project, Central Research Institute of Electric

Power Industry (CRIEPI)

JAPAN

SWART, Rob

Netherlands Environmental Assessment Agency

THE NETHERLANDS

TIRPAK, Dennis

International Institute for Sustainable Development (IISD)

USA

VOGEL, Coleen

Department of Geography, University of Witwatersrand SOUTH AFRICA

YOHE, Gary

Department of Economics, Wesleyan University

USA

\section{IV.2 Extended Writing Team member}

BARKER, Terry

Cambridge Centre for Climate Change Mitigation Research, University of Cambridge

UK 


\section{Annex V}

\section{List of Reviewers and Review Editors}

\section{V.1 Reviewers}

Consistent with IPCC Rules and Procedures, the draft SYR was sent for formal review to over 2,400 individual experts as well as to the 193 member governments of the IPCC. This appendix lists the individual experts (with affiliations at the time of submission of comments) and international organisations who submitted review comments on the draft SYR, and whose comments were considered by the Core Writing Team in its revision of the draft report.

Note: International organisations are listed at the end.

\section{Argentina \\ DEVIA, Leila \\ National Industrial Technology \\ TRAVASSO, María Isabel \\ Instituto Nacional de Tecnología \\ Agropecuaria}

WEHBE, Monica Beatriz

National University Rio Cuarto

\section{Australia \\ BARNETT, Jon \\ University of Melbourne \\ BINDOFF, Nathaniel \\ CSIRO MAR and University of \\ Tasmania}

BRUNSKILL, Gregg

Australian Institute of Marine Science

CHAMBERS, Lynda

Bureau of Meteorology Research Centre

CHURCH, John

CSIRO

JONES, Roger

CSIRO

KAY, Robert

Coastal Zone Management Pty Ltd

LOUGH, Janice

Australian Institute of Marine Science

MANTON, Michael

Monash University

SHEARMAN, David

University of Adelaide
WALKER, George

Aon Re Asia Pacific

WATKINS, Andrew

National Climate Centre, Australian

Bureau of Meteorology

WHITE, David

ASIT Consulting

YOUNUS, Aboul Fazal

Bangladesh Unnaya Parishad and The

University of Adelaide

Austria

CLEMENS, Torsten

OMV Exploration and Production

KASER, Georg

Institut fuer Geographie

University of Innsbruck

KIRCHENGAST, Gottfried

Wegener Center for Climate and Global

Change, University of Graz

MA, Tieju

International Institute for Applied

Systems Analysis

PAULI, Harald

University of Vienna and Austrian

Academy of Sciences

SCHRÖTER, Dagmar

Umweltbundesamt $\mathrm{GmbH}$

Belgium

KJAER, Christian

European Wind Energy Association

SAWYER, Steve

Global Wind Energy Council
VERHASSELT, Yola

Vrije Universiteit Brussel

Benin

YABI, Ibouraïma Fidele

Universite d Aborney-Calavi

Bolivia

HALLOY, Stephan

Conservation International

Brazil

AMBRIZZI, Tercio

University of São Paulo

BUSTAMANTE, Mercedes

University of Brasilia

GOMES, Marcos

Pontifical Catholic University of Rio de Janeiro

MOREIRA, José

Institute of Eletrotechnica and Energy

SANT'ANA, Silvio

Fundaçao Grupo Esquel Brasil

Bulgaria

YOTOVA, Antoaneta

National Institute of Meteorology and

Hydrology

Canada

AMIRO, Brian

University of Manitoba

BARBER, David

University of Manitoba

BELTRAMI, Hugo

St. Francis Xavier University 
BERRY, Peter

Health Canada

BRADY, Michael

Natural Resources Canada - Canadian

Forest Service

CHURCH, Ian

Yukon Government

CLARKE, R. Allyn

Fisheries and Oceans, Bedford Institute

of Oceanography

FISHER, David A

National Resources Canada

GRANDIA, Kevin

DeSmogBlog Society of British Colombia

HUPE, Jane

ICAO

JACKSON, David

McMaster Institute for Energy Studies

JANZEN, Henry

Agriculture and Agri-Food Canada

JEFFERIES, Robert

University of Toronto

LEMMEN, Donald

Natural Resources Canada

MICHAUD, Yves

Geological Survey of Canada

NYBOER, John

Simon Fraser University

SMITH, Sharon

Geological Survey of Canada

China

FANG, Xiuqi

Beijing Normal University

GUO, Xueliang

Institute of Atmospheric Physics, Chinese Academy of Sciences

LAM, Chiu-Ying

Hong Kong Observatory

REN, Guoyu

National Climate Center
SU, Jilan

Second Institute of Oceanography, State

Oceanic Administration

WANG, Bangzhong

China Meteorological Administration

YINGJIE, Liu

Institute of Environment and Sustainable

Development in Agriculture

ZHAO, Zong-Ci

China Meteorological Administration

ZHOU, Guangsheng

Institute of Botany, The Chinese

Academy of Sciences

Colombia

POVEDA, Germán

Universidad Nacional de Colombia

Cuba

DIAZ MOREJON, Cristobal Felix

Ministry of Science, Technology and the Environment

SUAREZ RODRIGUEZ, Avelino G. Institute of Ecology and Systematic, Agencia de Medio Ambiente

Czech Republic

HALENKA, Tomas

Faculty of Mathematics and Physics, Charles University, Prague

Denmark

ERHARD, Markus

European Environment Agency

MELTOFTE, Hans

National Environmental Research Institute, University of Aarhus

PORTER, John R.

University of Copenhagen

El Salvador MUNGUÍA DE AGUILAR, Martha Yvette

Ministry of Environment and Natural Resources

France

CAMPBELL, Nick

ARKEMA SA
CANEILL, Jean-Yves

Electricité de France

DE T'SERCLAES, Philippine

International Energy Agency

DOUGUÉDROIT, Annick

Université de Provence

HEQUETTE, Arnaud

Université du Littoral Côte d'Opale

LENOTRE, Nicole

Bureau de recherches géologiques et minières

MUIRHEID, Ben

International Fertilizer Trade Association

PHILIBERT, Cédric

International Energy Agency

PLANTON, Serge

Météo-France

RILLING, Jacques

Center Scientifique et Technique du

Bätiment

RUFFING, Kenneth

Germany

BRUCKNER, Thomas

Technical University of Berlin

GERTEN, Dieter

Potsdam Institute for Climate Impact

Research

GRASSL, Hartmut

Max Planck Institute for Meteorology

KUCKSHINRICHS, Wilhelm

Research Centre Juelich

LAWRENCE, Mark

Max Planck Institute for Chemistry

MATZARAKIS, Andreas

Meteorological Institute, University of Freiburg

MUELLER, Rolf

Research Centre Juelich

SCHWARZER, Klaus

Institute of Geosciences, University of Kiel 
TREBER, Manfred

Germanwatch

WALTHER, Gian-Reto

University of Bayreuth

WELP, Martin

University of Applied Sciences,

Eberswalde

WILLEBRAND, Jürgen

Leibniz Institut für

Meereswissenschaften

WINDHORST, Wilhelm

Ecology Centre, Kiel University

WURZLER, Sabine

North Rhine Westphalia State Agency

for Nature, Environment and Consumer

Protection

Hungary

BÉLA, Nováky

Szent István University

SOMOGYI, Zoltán

Hungarian Forest Research Institute

India

ROY, Joyashree

Jadavpur University

SHARMA, Upasna

Indian Institute of Technology, Bombay

SRIKANTHAN, Ramachandran

Physical Research Laboratory

Ireland

FINNEGAN, Pat

Greenhouse Ireland Action Network

TOL, Richard

Economic and Social Research Institute

Italy

CASERINI, Stefano

Politecnico di Milano

MARIOTTI, Annarita

National Agency for New Technologies, Energy and the Environment

RIXEN, Michel

NATO Undersea Research Center
Jamaica

CLAYTON, Anthony

University of the West Indies

Japan

AKIMOTO, Keigo

Research Institute of Innovative Tech-

nology for the Earth

ALEXANDROV, Georgii

National Institute for Environmental

Studies

ANDO, Mitsuru

Toyama University of International

Studies

IKEDA, Motoyoshi

Hokkaido University

INOUE, Takashi

Tokyo University of Science

KOBAYASHI, Noriyuki

Nihon University (Law School)

KOBAYASHI, Shigeki

Toyota Research and Development

Laboratories, Inc.

KOIDE, Hitoshi

Waseda University

KOMIYAMA, Ryoichi

The Institute of Energy Economics, Japan

MARUYAMA, Koki

Central Research Institute of Electric

Power Industry

MASUI, Toshihiko

National Institute for Environmental

Studies

MATSUI, Tetsuya

Hokkaido Research Centre, Forestry and Forest Products Research Institute

MIKIKO, Kainuma

National Institute for Environmental

Studies

MORI, Shunsuke

Tokyo University of Science
MORISUGI, Hisayoshi

Japan Research Institute

NAKAKUKI, Shinichi

Tokyo Electric Power Company

NAKAMARU, Susumu

Sun Management Institute

ONO, Tsuneo

Hokkaido National Fisheries Research Institute, Fisheries Research Agency

YAMAGUCHI, Mitsutsune

The University of Tokyo

YOSHINO, Masatoshi

Kenya

DEMKINE, Volodymyr

UNEP

Mexico

OSORNIO VARGAS, Alvaro

Universidad Nacional Autónoma de México

Moldova

COROBOV, Roman

Modern Institute for Humanities

The Netherlands

BREGMAN, Bram

Netherlands Organisation of Applied

Research

BRINKMAN, Robert

MARCHAND, Marcel

Delft Hydraulics

MISDORP, Robbert

International CZM-Centre, Ministry of Transport, Public Works and Water

Management

SCHYNS, Vianney

Climate Change and Energy Efficiency, Utility Support Group

STORM VAN LEEUWEN, Jan Willem

Ceedata Consultancy

VAN NOIJE, Twan

Royal Netherlands Meteorological Institute 
WORRELL, Ernst

Ecofys

New Zealand

CRAMPTON, James

GNS Science

GRAY, Vincent

SCHALLENBERG, Marc

University of Otago

Nigeria

ANTIA, Effiom

University of Calabar

\section{Norway}

ERIKSEN, Siri

University of Oslo

HOFGAARD, Annika

Norwegian Institute for Nature Research

KRISTJANSSON, Jon Egill

University of Oslo

Peru

GAMBOA FUENTES, Nadia Rosa

Pontificia Universidad Catolica Del Peru

Philippines

OGAWA, Hisashi

World Health Organization Regional

Office for the Western Pacific

TIBIG, Lourdes

Philippine Atmospheric, Geophysical and Astronomical Services Administration

\section{Portugal}

DAS NEVES, Luciana

University of Porto

PAIVA, Maria Rosa

New University of Lisbon

RAMOS-PEREIRA, Ana

University of Lisbon

Republic of Korea

KIM, Suam

Pukyong National University

\section{Romania}

BORONEANT, Constanta

National Meteorological Administration
Russian Federation

GYTARSKY, Michael

Institute of Global Climate and Ecology

Saudi Arabia

ALFEHAID, Mohammed

Ministry of Petroleum

BABIKER, Mustafa

Saudi Aramco

South Africa

TANSER, Frank

Africa Centre for Health and Population

Studies

WINKLER, Harald

Energy Research Centre, University of

Cape Town

Spain

ALONSO, Sergio

Universitat de les Illes Balears

ANADÓN, Ricardo

Universidad de Oviedo

HERNÁNDEZ, Félix

IEG-CSIC

MARTIN-VIDE, Javier

Physical Geography University of

Barcelona

MORENO, Jose M.

Faculty of Environmental Sciences,

Universidad de Castilla-La Mancha

RIBERA, Pedro

Universidad Pablo de Olavide

RODRIGUEZ ALVAREZ, Dionisio

Xunta de Galicia

Sweden

LECK, Caroline

Department of Meteorology

MOLAU, Ulf

Göteborg University

MÖLLERSTEN, Kenneth

Swedish Energy Agency

RUMMUKAINEN, Markku

Swedish Meteorological and Hydrological Institute
WEYHENMEYER, Gesa

Swedish University of Agricultural

Sciences

Switzerland

APPENZELLER, Christof

Federal Office of Meteorology and

Climatology, MeteoSwiss

CHERUBINI, Paolo

WSL Swiss Federal Research Institute

FISCHLIN, Andreas

Terresterial Systems Ecology, ETH

Zurich

JUERG, Fuhrer

Agroscope Research Station ART

MAZZOTTI, Marco

ETH Zurich

ROSSI, Michel J.

Ecole Polytechnique Fédérale de

Lausanne

Thailand

HENOCQUE, Yves

Department of Fisheries

SCHIPPER, Lisa

Southeast Asia START Regional Centre,

Chulalongkorn University

Turkey

SENSOY, Serhat

Turkish State Meteorological Service

UK

ALLAN, Richard

University of Reading

BARKER, Terry

Cambridge Centre for Climate Change

Mitigation Research

CLAY, Edward

Overseas Development Institute

CONVEY, Peter

British Antarctic Survey

CRABBE, M. James C.

University of Bedfordshire

GILLETT, Nathan

University of East Anglia 
HAIGH, Joanna

Imperial College

HARRISON, Paula

Oxford University Centre for the

Environment

HAWKINS, Stephen

Marine Biological Association of the UK

JEFFERSON, Michael

World Renewable Energy Network and Congress

JONES, Chris

Met Office Hadley Centre

McCULLOCH, Archie

University of Bristol

MORSE, Andy

University of Liverpool

MUIR, Magdalena

Environmental and Legal Services Ltd.

PAAVOLA, Jouni

University of Leeds

RAVETZ, Joe

University of Manchester

SHINE, Keith

University of Reading

SIMMONS, Adrian

European Centre for Medium-Range

Weather Forecasts

SIVETER, Robert

International Petroleum Industry

Environmental Conservation Association

SMITH, Leonard Allen

London School of Economics

SPENCER, Thomas

University of Cambridge

SROKOSZ, Meric

National Oceanography Centre

STONE, Dáithí

University of Oxford
STREET, Roger

UK Climate Impacts Programmes,

Oxford University Centre for the

Environment

USHER, Michael

University of Stirling

WOODWORTH , Philip

Proudman Oceanographic Laboratory

USA

ANYAH, Richard

Rutgers University

ATKINSON, David

International Arctic Research Center,

University of Alaska, Fairbanks

BRIENO RANKIN, Veronica

GeoSeq International LLC

CHAPIN, III, F. Stuart

University of Alaska, Fairbanks

CLEMENS, Steven

Brown University

CROWLEY, Tom

Duke University

DELHOTAL, Katherine Casey

RTI International

EPSTEIN, Paul

Harvard Medical School

EVERETT, John

Ocean Associates, Inc.

FAHEY, David

NOAA Earth Science Research Laboratory

GURWICK, Noel

Carnegie Institution

HAAS, Peter

University of Massachusetts

HEGERL, Gabriele

Duke University

KIMBALL, Bruce

USDA, Agricultural Research Service
KNOWLTON, Kim

Columbia University

LEE, Arthur

Chevron Corporation

LIOTTA, Peter

Pell Center for International Relations and Public Policy

MACCRACKEN, Michael

Climate Institute

MALONE, Elizabeth L

Pacific Northwest National Laboratory

MASTRANDREA, Michael

Stanford University

MATSUMOTO, Katsumi

University of Minnesota

MATSUOKA, Kenichi

University of Washington

McCARL, Bruce

Texas A \& M University

MILLER, Alan

International Finance Corporation -

CESEF

MOLINARI, Robert

University of Miami

MORGAN, Jack

Crops Research Lab

MURPHY, Daniel

NOAA Earth System Research Laboratory

NADELHOFFER, Knute

University of Michigan

NEELIN, J. David

UCLA

OPPENHEIMER, Michael

Princeton University

PARK, Jacob

Green Mountain College

PARKINSON, Claire

NASA Goddard Space Flight Center 
ROBOCK, Alan

Rutgers University

SCHWING, Franklin

US Dept. of Commerce

SHERWOOD, Steven

Yale University

SIDDIQI, Toufiq

Global Environment and Energy in $21^{\text {st }}$ century
SIEVERING, Herman

University of Colorado

SOULEN, Richard

TRENBERTH, Kevin

National Centre for Atmospheric

Research

International Organisations

LLOSA, Silvia

International Strategy for Disaster

Reduction
McCULLOCH, Archie

International Chamber of Commerce

SIMS, Ralph

International Energy Agency

SINGER, Stephan

WWF International

STEFANSKI, Robert

World Meteorological Organization

YAN, Hong

World Meteorological Organization

\section{V.2 Review Editors}

The role of Review Editors is to ensure that all substantive expert and government review comments are afforded appropriate consideration by the Core Writing Team. Two Review Editors were appointed for each Topic of this Synthesis Report. They confirm that all comments were considered in accordance with IPCC procedures.

\section{Topic 1}

JALLOW, Bubu Pateh

Department of Water Resources

THE GAMBIA

\section{KAJFEŽ-BOGATAJ , Lučka}

University of Ljubljana

SLOVENIA

\section{Topic 2}

BOJARIU, Roxana

National Institute of Meteorology and Hydrology

ROMANIA

HAWKINS, David

Natural Resources Defence Council Climate Center

USA
Topic 3

DIAZ, Sandra

CONICET-Universidad Nacional de

Córdoba

ARGENTINA

LEE, Hoesung

SOUTH KOREA

Topic 4

ALLALI, Abdelkader

Ministry of Agriculture, Rural Development and Fishing

MOROCCO

ELGIZOULI, Ismail

Higher Council for Environment and Natural Resources

SUDAN
Topic 5

WRATT, David

National Institute of Water and Atmospheric Research

NEW ZEALAND

HOHMEYER, Olav

University of Flensburg

GERMANY

Topic 6

GRIGGS, Dave

Monash University

AUSTRALIA/UK

LEARY, Neil

International START Secretariat USA 


\section{Index}

A.

acidification (see ocean acidification)

adaptation $56,57,61,65,70,73$

adaptive capacity $52,56,61,64,65,70,73$

aerosols $38,39,44,45,73$

Africa 30, 44, 50, 72, 73

agriculture/crops $33,36,37,48-53,56,57$

anthropogenic

emissions $36,38,44,72$

warming $39,41,46,72$

Antarctica 39, 47, 73

Arctic 33, 52, 65, 72

Article 2 (of UNFCCC) 64

Asia $30,32,50$

Australia and New Zealand 32, 50

B.

barriers

to adaptation $56,57,65,70,73$

to mitigation $58,59,65,68,70,73$

behaviour pattern (see lifestyle)

C.

carbon capture and storage (CCS) 60, 68

carbon dioxide $\left(\mathrm{CO}_{2}\right)$

concentrations 37-39, 52, 67, 72

emissions 36, 44, 47, 58, 66, 67, 72

carbon leakage 59

carbon price 58, 59

Clean Development Mechanism (CDM) 62 climate

-carbon cycle coupling $38,45,67,73$

change (see climate change)

variability $30,33,40,41,56$

climate change

abrupt 53, 54, 65

after stabilisation of GHGs 46, 47, 66, $67,72,73$

and air pollution 59,70

and water 49,57

attribution 38, 39, 41, 72

beyond $21^{\text {st }}$ century $46,47,66,67$

definitions 30

drivers $36-38$

impacts (see impact)

irreversible 53, 54

observed 30, 31, 33

projections $45-47$

regional 30, 46, 47, 49

climate sensitivity $38,66,67,72,73$

climate system $30,36,37,39,45$

co-benefits 59, 64

coastal/of coasts

defence 56,57

flooding $33,48,50-53,57,65$ concentration

atmospheric $37,38,72$

$\mathrm{CO}_{2}$-equivalent $36,37,59,66,67$

constant 45,46

confidence interval 27

cooperation (international) 62

cost

of adaptation 56

(see mitigation)

(see social cost of carbon)

cyclones (tropical) 30, 46

D.

damages $33,51,53,64,65,69$

days

cold 30,40

hot 30,46

deforestation 36,61

developing countries $31,37,59$

development pathway 44, 50, 66, 70, 73

drought $30,41,48-51,53,56,65,72$

dust 38

dust storm 33

E.

economic development 44, 50, 56, 61, 64

ecosystems $31,48,51-54$

emissions 36

$\mathrm{CO}_{2}$-equivalent 44,58

pathway/trajectory 66,67

reduction (see mitigation)

scenario 44

energy

demand 53, 56, 60, 61

efficiency 57, 59, 60, 68

intensity 37,61

low-carbon sources of 58,68

nuclear 68

renewable 57, 60, 68

supply/generation 36, 44, 50, 59, 60, 68

equilibrium

sea level (thermal expansion) 66, 67

temperature 47, 66, 67

equity $61,62,64$

Europe 30, 32, 50

extinction 52, 48, 50, 54, 64

extremes 30, 40, 46, 52, 53, 56, 65, 72

F.

feedback $38,40,46,73$

climate-carbon cycle $38,45,54,67,73$

fire $33,48,50,51,53$

floods 72

coastal $33,48,50-53,57,65$

river $48-50,52,53,57$

food production/crops $48,51,64$ forestation 61

fossil fuels $36,37,44,59,60$

G.

glaciers $30,49,50,52,57,65$

Global Warming Potential (GWP) 36, 72

greenhouse gases (GHGs) 36, 37, 40, 69

concentrations $39,46,64,66,67$

emissions 36, 37, 44, 45, 56, 58, 66, 67, 72

greening (of vegetation) 33

Greenland 47, 65, 67, 73

Gross Domestic Product (GDP) 37, 44, 50, $59,62,69$

H.

hail storms 33

halocarbons 37

health $33,48,49,50,51,52,53,56,57,59$, $64,65,70,72$

heat wave $30,40,46,50,52,53,72$

hydrological cycle/systems 31, 41, 50

hydropower 50, 53, 59, 60

I.

ice

(on land/ice sheet/ice cap) 30,47, 53, 65,73

sea ice $30,31,33,38,46,52,65,72$

impact (of climate change)

avoided/reduced/delayed 69, 70

beneficial 48-50, 52

irreversible 53, 54

observed $31-33,41$

projected $48-53$

regional 50-52

sectoral 48, 49, 51

industry 48, 53, 59, 60, 61

inertia 66,67

infrastructure $48,49,52,53,56-58$, 64-66

K.

Kyoto Protocol 59, 62

L.

Latin America 44, 50

land use $37,40,41,49,57,60,68,72$

lifestyle 59,73

lightning 33

low-emissions/low-carbon technology 58-60, 68

M.

Mediterranean sea/basin 30, 49

megadelta $48,50,52,65,72$ 
meridional overturning circulation

(MOC) 33, 51, 54, 65

methane $\left(\mathrm{CH}_{4}\right)$ 36-38, 60, 72

Middle East 44

migration

bird 33, 52

fish 33

population 53

Millennium Development Goals (MDGs) 70

mitigation 56, 58-61

benefits $66,69,70$

costs 69

options 58-60, 73

policies $44,60,61$

portfolio $61,68,73$

potential 58,59

mortality $33,50,51,53,59$

multi-century warming 47, 64

N.

nights

cold nights $30,40,53$

hot nights $30,40,53$

nitrate 39

nitrous oxide $\left(\mathbf{N}_{2} \mathbf{O}\right)$ 36-38, 60, 72

non- $\mathrm{CO}_{2}$ gases/options 60,68

North America 32, 52

Northern Hemisphere 30, 31, 33, 40, 46, 72

O.

ocean

acidification 52

temperature/heat content 30

organic carbon 38,44

P.

per capita

emissions 37

income 37 pests (disturbances) 33, 48

polar

ice sheets $30,47,53,65,73$

regions 32, 52, 64

population growth 44

precipitation

heavy precipitation $30,41,46,49,53$

pattern $30,41,46,47,50,73$

R.

radiative forcing $36-39,45,46,67$

rainfall (see precipitation)

reasons for concern $64,65,72$

research

funding 68

RD\&D 61, 62, 68, 73

risk management 64,69

runoff $31,49,61$

S.

Sahel 30

sea ice (see ice)

sea level rise/change $30,33,40,45-49,53$, $65,67,72,73$

settlements $48,50,52,53,57$

small islands $48,52,, 65,72$

snow (cover/pack) 30, 31, 33, 46, 49, 50, $52,53,57,72$

social cost of carbon 69

society $26,48,49,53,56,58$

spillover effects 59

SRES

emissions 44, 45, 46, 58, 70, 72

storylines/pathways 44,70

stabilisation 46,61

levels 47, 59, 66, 67, 68, 69, 73

pathway $66,67,69$

storms 40, 46, 50, 51, 56

stress (multiple) 52, 56, 65

sulphur dioxide/sulphate 38,44 sustainable development $44,49,61,70,72,73$

T.

technological change $44,61,73$

technology 56, 58, 60, 61, 68, 73

investment 58-60, 68, 73

temperature

changes $30-32,39,40,45,46,51,64$, $66,67,69$

variability $30,40,41$

Third Assessment Report (TAR) 26, 30$32,38-40,44-46,50,56,59,61,62,64-$ 66,72

tornadoes 33

tourism 50, 53, 57

transport $36,53,57,59,60,62$

U.

UNFCCC 30, 36, 37, 62, 64

uncertainty

key uncertainty 72,73

terminology 27

V.

vulnerability $48,56,60,61,64,65,70$, 72, 73

key vulnerability 50, 64

W.

water

adaptation options 57

National Water Management Plan of

Bangladesh 56

stress 49-51, 53, 65

resources $49,52,53,56,57,64,72$

wind patterns 40,46 


\section{Publications by the Intergovernmental Panel on Climate Change}

\section{Assessment Reports}

Fourth Assessment Report

Climate Change 2007: The Physical Science Basis

Contribution of Working Group I to the Fourth Assessment Report

Climate Change 2007: Impacts, Adaptation and Vulnerability Contribution of Working Group II to the Fourth Assessment Report

Climate Change 2007: Mitigation of Climate Change

Contribution of Working Group III to the Fourth Assessment Report

Climate Change 2007: Synthesis Report

Contribution of Working Groups I, II and III to the Fourth Assessment Report

Third Assessment Report

Climate Change 2001: The Scientific Basis

Contribution of Working Group I to the Third Assessment Report

Climate Change 2001: Impacts, Adaptation and Vulnerability Contribution of Working Group II to the Third Assessment Report

Climate Change 2001: Mitigation

Contribution of Working Group III to the Third Assessment Report

Climate Change 2001: Synthesis Report

Contribution of Working Groups I, II and III to the Third Assessment Report

\section{Second Assessment Report}

Climate Change 1995: The Science of Climate Change

Contribution of Working Group I to the Second Assessment Report

Climate Change 1995: Scientific-Technical Analyses of Impacts, Adaptations and Mitigation of Climate Change

Contribution of Working Group II to the Second Assessment Report

Climate Change 1995: The Economic and Social Dimensions of Climate Change

Contribution of Working Group III to the Second Assessment Report

Climate Change 1995: Synthesis of Scientific-Technical Information Relevant to Interpreting Article 2 of the UN Framework Convention on Climate Change

Contribution of Working Groups I, II and III to the Second Assessment Report

Supplementary Report to the First Assessment Report

Climate Change 1992: The Supplementary Report to the IPCC Scientific Assessment

Supplementary report of the IPCC Scientific Assessment Working Group I
Climate Change 1992: The Supplementary Report to the IPCC Impacts Assessment

Supplementary report of the IPCC Impacts Assessment Working Group II

Climate Change: The IPCC 1990 and 1992 Assessments

IPCC First Assessment Report Overview and Policymaker Summaries and 1992 IPCC Supplementary Report

\section{First Assessment Report}

Climate Change: The Scientific Assessment

Report of the IPCC Scientific Assessment Working Group I, 1990

Climate Change: The IPCC Impacts Assessment

Report of the IPCC Impacts Assessment Working Group II, 1990

Climate Change: The IPCC Response Strategies

Report of the IPCC Response Strategies Working Group III, 1990

\section{Special Reports}

Carbon Dioxide Capture and Storage 2005

Safeguarding the Ozone Layer and the Global Climate System: Issues Related to Hydrofluorocarbons and Perfluorocarbons (IPCC/TEAP joint report) 2005

Land Use, Land-Use Change and Forestry 2000

Emissions Scenarios 2000

Methodological and Technological Issues in Technology Transfer 2000

Aviation and the Global Atmosphere 1999

The Regional Impacts of Climate Change: An Assessment of Vulnerability 1997

Climate Change 1994: Radiative Forcing of Climate Change and an Evaluation of the IPCC IS92 Emissions Scenarios 1994

\section{Methodology Reports and technical guidelines}

2006 IPCC Guidelines for National Greenhouse Gas Inventories (5 Volumes) 2006

Definitions and Methodological Options to Inventory Emissions from Direct Human-induced Degradation of Forests and Devegetation of Other Vegetation Types 2003

Good Practice Guidance for Land Use, Land-use Change and Forestry IPCC National Greenhouse Gas Inventories Programme, 2003

Good Practice Guidance and Uncertainty Management in National Greenhouse Gas Inventories IPCC National Greenhouse Gas Inventories Programme, 2000

Revised 1996 IPCC Guidelines for National Greenhouse Gas Inventories (3 volumes), 1996 
IPCC Technical Guidelines for Assessing Climate Change Impacts and Adaptations 1995

IPCC Guidelines for National Greenhouse Gas Inventories (3 volumes) 1994

Preliminary Guidelines for Assessing Impacts of Climate Change 1992

Assessment of the Vulnerability of Coastal Areas to Sea Level Rise - A Common Methodology 1991

\section{Technical Papers}

Climate Change and Biodiversity

IPCC Technical Paper 5, 2002

Implications of Proposed $\mathrm{CO}_{2}$ Emissions Limitations

IPCC Technical Paper 4, 1997

Stabilisation of Atmospheric Greenhouse Gases: Physical, Biological and Socio-Economic Implications

IPCC Technical Paper 3, 1997
An Introduction to Simple Climate Models Used in the IPCC Second Assessment Report

IPCC Technical Paper 2, 1997

Technologies, Policies and Measures for Mitigating Climate Change

IPCC Technical Paper 1, 1996

\section{Supplementary material}

Global Climate Change and the Rising Challenge of the Sea Coastal Zone Management Subgroup of the IPCC Response Strategies Working Group, 1992

Emissions Scenarios

Prepared by the IPCC Response Strategies Working Group, 1990

For a more comprehensive list of supplementary material published by the IPCC (workshop and meeting reports), please see www.ipcc.ch or contact the IPCC Secretariat. 
